SÉRGIO DALMAS

\title{
Obtenção de Autovalores de Soluções em Série de Problemas de Condução de Calor com Condições de Contorno Convectivas
}


UNIVERSIDADE ESTADUAL DE CAMPINAS

FACULDADE DE ENGENHARIA MECÂNICA

\author{
SÉRGIO DALMAS
}

\title{
Obtenção de Autovalores de Soluções em Série de Problemas de Condução de Calor com Condições de Contorno Convectivas
}

Tese de Doutorado apresentada à Faculdade de Engenharia Mecânica da Universidade Estadual de Campinas como parte dos requisitos exigidos para obtenção do título de Doutor em Engenharia Mecânica, na Área de Térmica e Fluidos.

Orientador: Prof. Dr. Luiz Fernando Milanez

ESTE EXEMPLAR CORRESPONDE A VERSÃO FINAL DA TESE DEFENDIDA PELO ALUNO SÉRGIO DALMAS, E ORIENTADA PELO PROF. DR. LUIZ FERNANDO MILANEZ

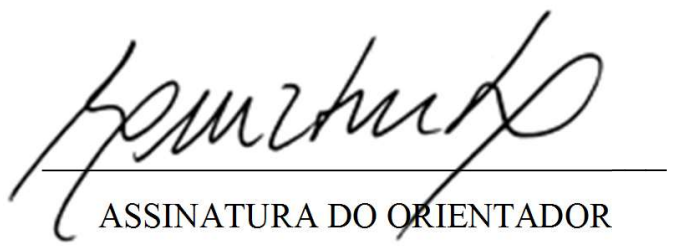

CAMPINAS 
Agência de fomento: Não se aplica N⿳a do processo: Não se aplica

Ficha Catalográfica

Universidade Estadual de Campinas

Biblioteca da Área de Engenharia e Arquitetura

Elizangela Aparecida dos Santos Souza - CRB 8/8098

D168o Dalmas, Sérgio, 1964- Obtenção de autovalores de soluções em série de problemas de condução de calor com condições de contorno convectivas / Sérgio Dalmas. - Campinas, SP: [s.n.], 2015.

Orientador: Luiz Fernando Milanez

Tese (doutorado) - Universidade Estadual de Campinas, Faculdade de Engenharia Mecânica.

1. Chebyshev, Aproximação de. 2. Padé, Aproximação de. 3. Raízes. 4. Autovalores. 5. Séries. I Milanez, Luiz Fernando, 1980-. II. Universidade Estadual de Campinas. Faculdade de Engenharia Mecânica. III. Título.

Informações para a Biblioteca Digital

Título em Inglês: Obtaining eigenvalues of series solutions of heat conduction problems with convective boundary conditions

Palavras-chave em Inglês:

Chebyshev, Approach

Padé, Approach

Roots

Eigenvalues

Series

Área de concentração: Térmica e Fluidos

Titulação: Doutor em Engenharia Mecânica

Banca examinadora:

Luiz Fernando Milanez [Orientador]

Carlos Alberto Carrasco Altemani

William Roberto Wolf

José Roberto Simões Moreira

Oscar Maurício Hernandez Rodrigues

Data da defesa: 25-08-2015

Programa de Pós-Graduação: Engenharia Mecânica 


\section{UNIVERSIDADE ESTADUAL DE CAMPINAS}

FACULDADE DE ENGENHARIA MECÂNICA

COMISSÃO DE PÓS-GRADUAÇÃO EM ENGENHARIA MECÂNICA

DEPARTAMENTO DE ENERGIA

TESE DE DOUTORADO

\section{Obtenção de Autovalores de Soluções em Série de Problemas de Condução de Calor com Condições de Contorno Convectivas}

Autor: Sérgio Dalmas

Orientador: Prof. Dr. Luiz Fernando Milanez

A Banca Examinadora composta pelos membros abaixo aprovou esta Tese:

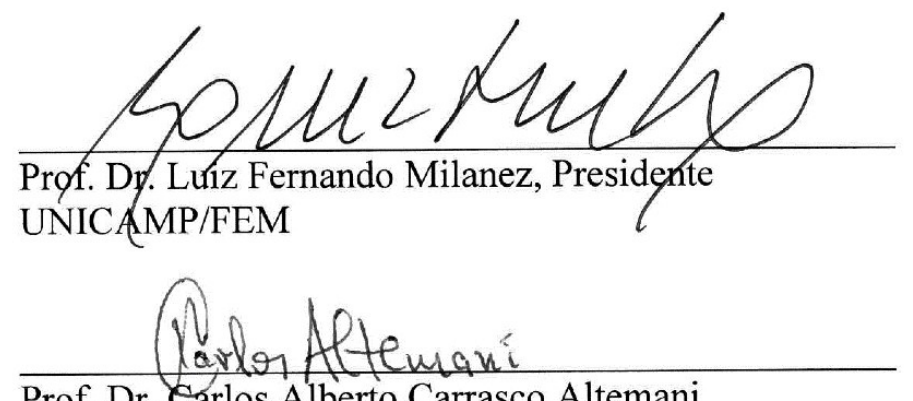

Prof. Dr. Carlos Alberto Carrasco Altemani

UNICAMP/FEM

Will w w w w

Prof. Dr. William Roberto Wolf

UNICAMP/FEM

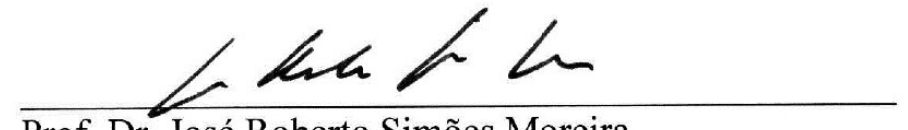

Prof. Dr. José Roberto Simões Moreira USP/EPUSP

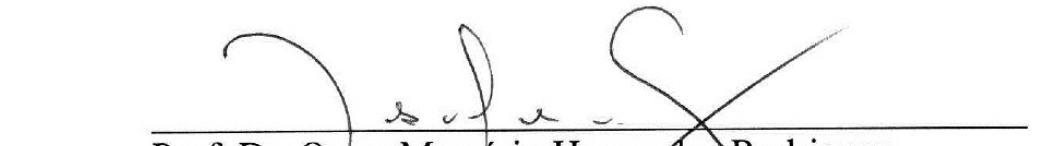

Prof. Dr. Oscar Maurício Hernapdez Rodriguez

USP/EESC 


\section{Agradecimentos}

Agradeço ao professor Milanez não só pela honra de ter agora seu nome como meu orientador de doutorado, nome este reconhecido internacionalmente como de grande pesquisador da área de Condução de Calor, mas também por isto se traduzir em uma orientação com um olhar superior, abrangente e de alto nível;

Agradeço ao professor Altemani por suas participações no meu trabalho, grande exemplo de

professor no Departamento de Engenharia Mecânica da Unicamp, sempre citado como referência pelos alunos que tiveram oportunidade de conhece-lo;

Agradeço ao professor Ganzarolli pela sua participação no exame de qualificação, pelas ideias, sugestões e comentários do projeto da minha tese, demonstrando plena lucidez pelo meu trabalho;

Por fim, agradeço ao meu pai, Aldacyr João, a quem dedico esta tese, por não ter perdido, nem por um momento, confiança pelo que eu poderia realizar. 


\section{Resumo}

Excluídos problemas simples de condução de calor nos quais a temperatura depende apenas do tempo ou apenas de uma coordenada de posição, os demais levam a equações diferenciais parciais, as quais tem soluções em termos de séries obtidas de vários métodos como a separação de variáveis, a superposição, a função de Green, a técnica da transformada integral, a transformada de Laplace e o teorema de Duhamel. Estas soluções dependem de autovalores que são obtidos das raízes de equações transcendentais que na maioria dos casos não podem ser expressas em forma fechada, mas podem ser obtidas de tabelas, expressões aproximadas, e expressões iterativas. 0 objetivo desse estudo é encontrar novas expressões para estas raízes, que sejam mais simples ou que tenham mais exatidão do que as já existentes. As três equações transcendentais que são consideradas aqui são as mais frequentemente utilizadas entre as que não tem solução fechada, e surgem quando as condições de contorno são convectivas. Uma nova família de funções iterativas é obtida, a qual inclui várias funções clássicas e, em particular, toda a família de métodos de Householder. Um novo método obtido é o que tem convergência mais rápida para as presentes equações. Apesar das tabelas de raízes apresentarem valores com vários dígitos significativos, problemas reais dificilmente levam a um valor da variável independente que pode ser diretamente encontrado, tornando-se necessário o uso de interpolação. Então, a exatidão de raízes obtidas por estas tabelas é limitada pela exatidão da interpolação, a qual pode ser comparada com a das expressões aproximadas. As expressões existentes são analisadas utilizando propriedades das raízes. Uma expressão aproximada desenvolvida para a primeira raiz das três equações é baseada no método do ponto fixo, outra é obtida da aplicação do conceito de MiniMax para se reajustar expressões de outros autores, e uma final tem forma algébrica. O conceito de MiniMax não é obtido através de algum método que possa ser considerado elementar, e dois novos métodos são desenvolvidos para aplicá-lo. Modernos sistemas algébricos computacionais são utilizados para gerar novas expressões aproximadas para a primeira raiz, mas encontrou-se que elas podem ser melhoradas através de métodos analíticos. Expansão em frações contínuas e novamente a aproximação de Padé são utilizadas para se obter expressões de grande exatidão. Expressões que levam a bons resultados para a primeira raiz são generalizadas para que elas sirvam para as demais raízes. Finalmente, uma comparação é feita considerando todas expressões aproximadas, indicando quais são consideradas as melhores.

Palavras Chave: aproximação de Chebyshev, aproximação de Padé, raízes, autovalores, séries. 


\section{Abstract}

Apart from simple problems of heat conduction in which the temperature depends only on the time or just on a position coordinate, the others lead to partial differential equations, which have solutions in terms of series obtained from various methods such as separation variables, superposition, the Green's function, the technique of integral transform, the Laplace transform and Duhamel's theorem. These solutions depend on eigenvalues, which are obtained from the roots of transcendental equations that in most cases cannot be expressed in closed form, but they can be obtained from tables, approximate expressions and iterative expressions. The objective of this study is to find new expressions for these roots, which are simpler or have more accuracy than the existing ones. The three transcendental equations that are considered here are the most frequently used among those that have not closed solution, and appear when the boundary conditions are convective. A new family of iterative functions is proposed, which includes several classical functions and, in particular, the entire family of Householder methods. A new method is obtained which has faster convergence to the present equations. Although the tables of roots present values with various significant digits, real problems hardly lead to a value of the independent variable that can be directly found, making it necessary to use interpolation. Then, the accuracy of the roots obtained from these tables is limited by the accuracy of the interpolation, which can be compared with the approximate expressions. Existing expressions are analyzed using the root properties. An approximate expression developed for the first root of the three equations is based on the fixed point method, another is obtained from the application of the concept of MiniMax to readjust expressions of others authors, and the last one has an algebraic form. The MiniMax concept is not obtained through any method that can be considered elementary, and two new methods are developed to apply it. Modern computer algebra systems are used to generate new approximate expressions for the first root, but it is found that they can be improved by analytical methods. Expansion in continuous fractions is adopted and the Padé approximation to obtain expressions of greater accuracy. Expressions leading to good results for the first root are generalized so that they serve for the other roots. Finally, a comparison is made considering all approximate expressions, indicating what are considered the best.

Keywords: Chebyshev approach, Padé approach, roots, eigenvalues, series 


\section{Lista de llustrações}

Figura 1.1 Vista de paralelepípedo com a face frontal correspondente a uma das superfícies com dimensões pequenas ou isoladas, e com face esquerda com temperatura nula 5

Figura 1.2 Vista de paralelepípedo com a face frontal correspondente a uma das superfícies com dimensões pequenas ou isoladas, e com face esquerda isolada.

Figura 1.3 Vista de paralelepípedo com a face frontal correspondente a uma das superfícies com dimensões maiores ou não isoladas 7

Figura 1.4 Cilindro com bases bem menores do que a superfície lateral ......................................... 8

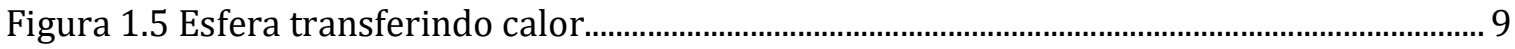

Figura 2.1 Abscissas correspondentes às quatro primeiras raízes da Eq. (1.5)...........................19

Figura 2.2 Abscissas correspondentes às quatro primeiras raízes da Eq. (1.6)..........................19

Figura 2.3 Abscissas correspondentes às quatro primeiras raízes da Eq. (1.7)..........................19

Figura 2.4 Curvas correspondentes às quatro primeiras raízes da Eq. (1.5) ...............................20

Figura 2.5 Curvas correspondentes às quatro primeiras raízes da Eq. (1.6) ...............................20

Figura 2.6 Curvas correspondentes às quatro primeiras raízes da Eq. (1.7) ...............................20

Figura 2.7 Valores aproximados obtidos pela Eq. (2.14) de Luikov (1968) e valores exatos da primeira raiz das Eqs. (1.5), (1.6) e (1.7) 25

Figura 2.8 Erro dos valores obtidos pela Eq. (2.14) de Luikov (1968) para a primeira raiz das Eqs. (1.5), (1.6) e (1.7) . .26

Figura 2.9 Erro relativo dos valores obtidos pela Eq. (2.14) de Luikov (1968) para a primeira raiz das Eqs. (1.5), (1.6) e (1.7) .26

Figura 2.10 Valores aproximados obtidos pela Eq. (2.20) de Stevens e Luck (1999) e valores exatos (representados por linhas pontilhadas) das quatro primeiras raízes da Eq. (1.5)... 37

Figura 4.1 Erro relativo dos valores de $\beta 1$ obtidos através de interpolação linear da Tabela 4.1 para $0,001 \leq \mathrm{c} \leq 100$ 57

Figura 4.2 Erro relativo dos valores de $\beta 1$ obtidos através de interpolação linear da Tabela 4.1 para $0 \leq \mathrm{c} \leq 0,001$. .58

Figura 5.1 Ponto de intersecção correspondente à primeira raiz da Eq. (1.5) 66

Figura 5.2 Erro relativo dos valores obtidos pela Eq. (2.28) de Ostrogorsky (2010), e dos valores obtidos pela mesma equação com parâmetros calculados pelo critério MiniMax. .71

Figura 5.3 Erro relativo das raízes da Eq. (1.5) calculadas pela Eq. (5.4) 72 
Figura 5.4 Erro relativo das raízes da Eq. (1.5) calculadas pela Eq. (5.4) com o eixo de $c$ perpendicular à figura

Figura 5.5 Erro relativo das raízes da Eq. (1.5) calculadas pela Eq. (5.4) para três valores do parâmetro de ajuste $r$ .73

Figura 5.6 Erro relativo das raízes da Eq. (1.5) calculadas pela Eq. (5.4) para o valor ótimo do parâmetro de ajuste $r$ . .74

Figura 5.7 Erro relativo das raízes da Eq. (1.6) calculadas pela Eq. (5.4)....................................75

Figura 5.8 Erro relativo das raízes da Eq. (1.7) calculadas pela Eq. (5.4) 75

Figura 5.9 Erro relativo das raízes da Eq. (1.5) calculadas pela primeira forma da expressão algébrica

Figura 6.1 Erro relativo das raízes da Eq. (1.5) calculadas pela primeira forma da expressão racional gerada pelos sistemas algébricos computacionais

Figura 6.2 Erro relativo das raízes da Eq. (1.5) calculadas pela expressão algébrica obtida indiretamente da expressão racional pelos sistemas algébricos computacionais

Figura 6.3 Erro relativo das raízes da Eq. (1.5) calculadas pela expressão algébrica obtida indiretamente da expressão racional de ordem $1 / 1$ gerada pelos sistemas algébricos computacionais

Figura 6.4 Erro relativo das raízes da Eq. (1.5) calculadas pela expressão algébrica obtida indiretamente da expressão racional de ordem $1 / 2$ gerada pelos sistemas algébricos computacionais

Figura 6.5 Erro relativo das raízes da Eq. (1.5) calculadas pela expressão algébrica obtida indiretamente da expressão racional de ordem 2/1 gerada pelos sistemas algébricos computacionais

Figura 6.6 Erro relativo das raízes da Eq. (1.5) calculadas pela expressão algébrica obtida indiretamente da expressão racional de ordem $2 / 2$ gerada pelos sistemas algébricos computacionais

Figura 6.7 Aproximação da razão entre as funções de Bessel por fração contínua .89

Figura 7.1 Localização dos melhores valores do parâmetro $x 6$ para a expressão para as raízes Eq. (1.5) obtida a partir de Padé 1,1

Figura 7.2 Expressão obtida a partir de Padé 1,1 para as raízes Eq. (1.5) com $x 2=0,575$ e $x 6=1,59$ 100

Figura 7.3 Expressão obtida a partir de Padé 1,1 para as raízes Eq. (1.5) com $x 2=2,286$ e $x 6=3,83$ 100 
Figura 7.4 Expressão obtida a partir de Padé 1,1 para as raízes Eq. (1.5) com os parâmetros

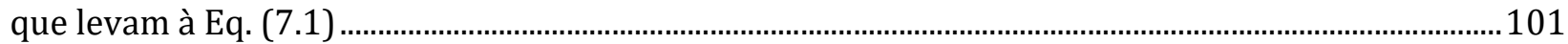

Figura 8.1 Expressões de muito menor exatidão para a primeira raiz da Eq. (1.5) .................111

Figura 8.2 Expressões de muito menor exatidão para a primeira raiz da Eq. (1.6) .................111

Figura 8.3 Expressões de muito menor exatidão para a primeira raiz da Eq. (1.7) .................111

Figura 8.4 Expressões de menor exatidão para a primeira raiz da Eq. (1.5)...............................114

Figura 8.5 Expressões de menor exatidão para a primeira raiz da Eq. (1.6)..............................114

Figura 8.6 Expressões de menor exatidão para a primeira raiz da Eq. (1.7)...............................114

Figura 8.7 Expressões de intermediária exatidão para a primeira raiz da Eq. (1.5)................ 116

Figura 8.8 Expressões de intermediária exatidão para a primeira raiz da Eq. (1.6)................ 116

Figura 8.9 Expressões de intermediária exatidão para a primeira raiz da Eq. (1.7)................116

Figura 8.10 Expressões de maior exatidão para a primeira raiz da Eq. (1.5) .............................118

Figura 8.11 Expressões de maior exatidão para a primeira raiz da Eq. (1.7) .............................118

Figura 8.12 Expressões de maior exatidão para a primeira raiz da Eq. (1.7) .............................118

Figura 8.13 Expressões de muito maior exatidão para a primeira raiz da Eq. (1.5) ................120

Figura 8.14 Expressões de muito maior exatidão para a primeira raiz da Eq. (1.7) ................120

Figura 8.15 Expressões de muito maior exatidão para a primeira raiz da Eq. (1.7) ................120

Figura 8.16 Expressão de extrema exatidão para a primeira raiz das Eqs. (1.5), (1.6) e (1.7)

Figura 8.17 Expressão de extrema exatidão para a primeira raiz das Eqs. (1.5), (1.6) e (1.7)

Figura 8.18 Expressões de menor exatidão para a segunda raiz da Eq. (1.5)............................124

Figura 8.19 Expressões de menor exatidão para a quarta raiz da Eq. (1.5) ................................124

Figura 8.20 Expressões de menor exatidão para a oitava raiz da Eq. (1.5) .................................124

Figura 8.21 Expressões de menor exatidão para a segunda raiz da Eq. (1.6)............................125

Figura 8.22 Expressões de menor exatidão para a segunda raiz da Eq. (1.6).............................125

Figura 8.23 Expressões de menor exatidão para a oitava raiz da Eq. (1.6) .................................125

Figura 8.24 Expressões de menor exatidão para a segunda raiz da Eq. (1.7)............................126

Figura 8.25 Expressões de menor exatidão para a quarta raiz da Eq. (1.7) ...............................126

Figura 8.26 Expressões de menor exatidão para a oitava raiz da Eq. (1.7) ...............................126

Figura 8.27 Expressões de maior exatidão para a segunda raiz da Eq. (1.5) ..............................129

Figura 8.28 Expressões de maior exatidão para a quarta raiz da Eq. (1.5).................................129

Figura 8.29 Expressões de maior exatidão para a oitava raiz da Eq. (1.5)..................................129 
Figura 8.30 Expressões de maior exatidão para a segunda raiz da Eq. (1.6) .............................130

Figura 8.31 Expressões de maior exatidão para a quarta raiz da Eq. (1.6).................................130

Figura 8.32 Expressões de maior exatidão para a oitava raiz da Eq. (1.6)..................................130

Figura 8.33 Expressões de maior exatidão para a segunda raiz da Eq. (1.7) .............................131

Figura 8.34 Expressões de maior exatidão para a quarta raiz da Eq. (1.7).................................131

Figura 8.35 Expressões de maior exatidão para a oitava raiz da Eq. (1.7)..................................131 


\section{Lista de Tabelas}

Tabela 2.1 Valores limites para as raízes das Eqs. (1.5), (1.6) e (1.7) ..............................................21

Tabela 3.1 Compilação de funções iterativas de um ponto................................................................46

Tabela 3.2 Cologaritmo do módulo do erro relativo máximo das raízes de várias equações após

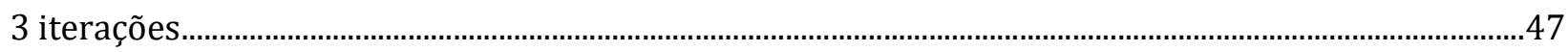

Tabela 3.3 Erro relativo máximo de raízes da Eq. (1.5) após 2 iterações........................................49

Tabela 3.4 Erro relativo máximo de raízes da Eq. (1.6) após 2 iterações........................................50

Tabela 3.5 Erro relativo máximo de raízes da Eq. (1.7) após 2 iterações..........................................50

Tabela 3.6 Erro relativo máximo da função iterativa da Eq. (3.23) com valores inicias dados pelas Eqs. (3.34) e (3.33) e coeficientes dados pelas Eqs. (3.38) a (3.44) ….............................................51

Tabela 4.1 Seis Primeiras Raízes da Eq. (1.5) ……….........................................................................54

Tabela 4.2 Seis Primeiras Raízes da Eq. (1.6) ....................................................................................55

Tabela 4.3 Seis Primeiras Raízes da Eq. (1.7)......................................................................................56

Tabela 4.4 Máximos módulos do erro relativo das raízes da Eq. (1.5) obtidas através de

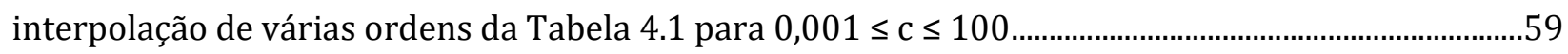

Tabela 4.5 Máximos módulos do erro relativo das raízes da Eq. (1.6) obtidas através de interpolação de várias ordens da Tabela 4.2 para $0,01 \leq \mathrm{c} \leq 100$ 59

Tabela 4.6 Máximos módulos do erro relativo das raízes da Eq. (1.7) obtidas através de

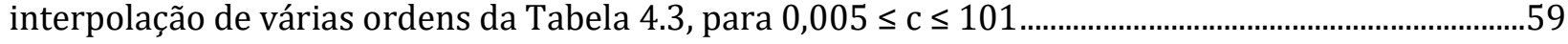

Tabela 4.7 Nove Primeiras Raízes da Eq. (1.5) ......................................................................................61

Tabela 4.8 Cinco Primeiras Raízes da Eq. (1.6)...................................................................................62

Tabela 4.9 Nove Primeiras Raízes da Eq. (1.7) ...................................................................................62

Tabela 4.10 Máximos módulos do erro relativo das raízes obtidas através de interpolação linear da Tabela 4.7.

Tabela 4.11 Máximos módulos do erro relativo das raízes obtidas através de interpolação da Tabela 4.7, considerando todo o intervalo $c>0$ (ou $c \geq 0,2$ no caso de interpolação de $1^{\underline{a}}$ ordem de $\beta 1)$

Tabela 4.12 Máximos módulos do erro relativo das raízes obtidas através de interpolação da Tabela 4.8, considerando todo o intervalo $c>0$ (ou $c \geq 0,02$ no caso de interpolação de 1 a ordem de $\beta 1)$ 
Tabela 4.13 Máximos módulos do erro relativo das raízes obtidas através de interpolação da Tabela 4.9, considerando todo o intervalo $c>0$ (ou $c \geq 0,05$ no caso de interpolação de $1 \stackrel{\text { a }}{\text { ordem }}$ de $\beta 1)$ 65

Tabela 5.1 Resultados do algoritmo de troca de Remez para a primeira raiz da Eq. (1.5) calculada pela primeira forma da expressão algébrica.

Tabela 5.2 Resultados do algoritmo de troca de Remez para a primeira raiz da Eq. (1.6) calculada pela primeira forma da expressão algébrica.

Tabela 5.3 Resultados do algoritmo de troca de Remez para a primeira raiz da Eq. (1.7) calculada pela primeira forma da expressão algébrica.

Tabela 6.1 Resultados do algoritmo de troca de Remez para a primeira raiz da Eq. (1.5) calculada pela Eq. (6.12)

Tabela 6.2 Resultados do algoritmo de troca de Remez para a primeira raiz da Eq. (1.6) calculada pela Eq. (6.12) . .97

Tabela 6.3 Resultados do algoritmo de troca de Remez para a primeira raiz da Eq. (1.7) calculada pela Eq. (6.12)

Tabela 7.1 Busca de expressão para um parâmetro ótimo da expressão de Yovanovich MiniMax Generalizada 104

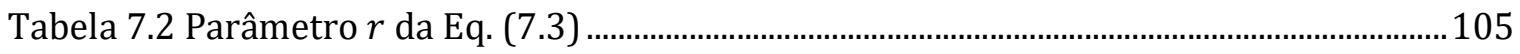

Tabela 7.3 Parâmetros $k$ e $A$ da Eq. (7.4) ……….............................................................................106 


\section{Lista de Símbolos}

\section{Letras Latinas}

$a_{i} \quad i$-ésimo coeficiente do numerador da aproximação de Padé

A Coeficiente

$A \quad$ Área $\left[\mathrm{m}^{2}\right]$

$A_{s, a} \quad$ Área da superfície sobre a qual é aplicada fluxo de calor $\left[\mathrm{m}^{2}\right]$

$A_{s, c} \quad$ Área da superfície que transfere calor por convecção $\left[\mathrm{m}^{2}\right]$

$A_{s, r} \quad$ Área da superfície que transfere calor por radiação $\left[\mathrm{m}^{2}\right]$

$A_{\text {sup }}$ Área da superfície $\left[\mathrm{m}^{2}\right]$

$A_{t r} \quad$ Área da seção transversal $\left[\mathrm{m}^{2}\right]$

b $\quad$ Raio [m]

$b_{i} \quad i$-ésimo coeficiente do denominador da aproximação de Padé

$B \quad$ Coeficiente

Bi Número de Biot

c Calor específico $[\mathrm{J} / \mathrm{kg} \cdot \mathrm{K}]$

c Variável independente das equações transcendentais

$c_{i} \quad i$-ésimo coeficiente do polinômio de Taylor

C Coeficiente

D Coeficiente

E Coeficiente

$f(x)$ Função $f$ da variável independente $x$

$f_{i} \quad$ i-ésimo parâmetro $\Pi$

Fo Número de Fourier (adimensional)

G Função de Green $\left[{ }^{\circ} \mathrm{C}\right]$ ou $[\mathrm{K}]$

$h \quad$ Coeficiente de transferência de calor por convecção $\left[\mathrm{W} / \mathrm{m}^{2} \cdot \mathrm{K}\right]$

$k \quad$ Condutividade térmica $[\mathrm{W} / \mathrm{m} \cdot \mathrm{K}]$

$H \quad$ Razão entre o coeficiente de convecção e a condutividade térmica do meio [m-1]

$L \quad$ Comprimento de uma das arestas de um paralelepípedo [m]

$L_{c} \quad$ Comprimento característico [m]

m 1, 2 e 3 para as Eqs. (1.5), (1.6) e (1.7), respectivamente 


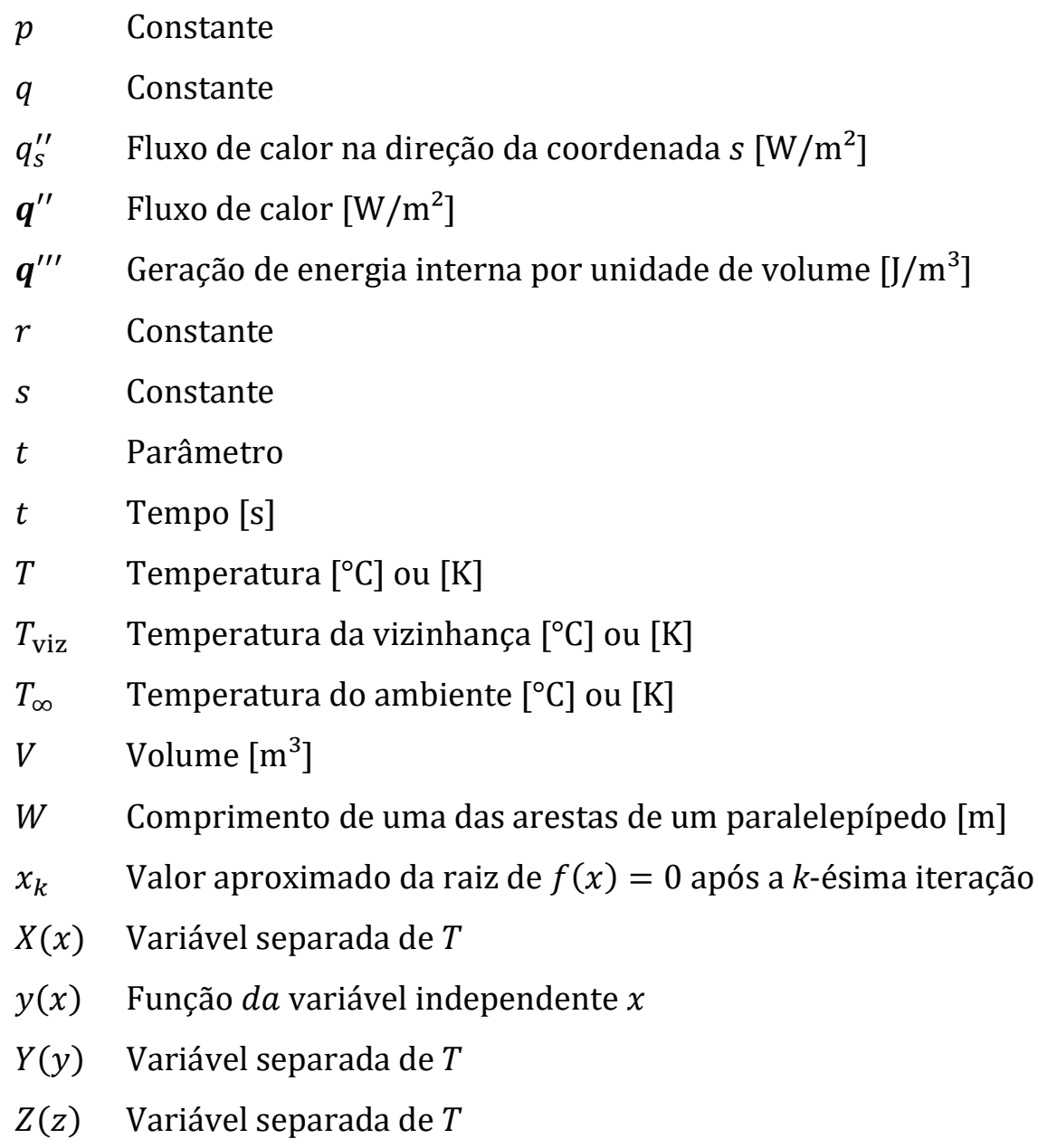

\section{Letras Gregas}

$\alpha \quad$ Difusividade térmica $\left[\mathrm{m}^{2} / \mathrm{s}\right]$

$\beta \quad$ Raiz da equação transcendental $\left[\mathrm{m}^{-1}\right]$

$\beta_{n} \quad n$-ésima raiz da equação transcendental ou seu valor aproximado [m-1]

$\beta_{n}^{k} \quad$ Valor aproximado da $n$-ésima raiz após a $k$-ésima iteração $\left[\mathrm{m}^{-1}\right]$

$\beta_{n, 0} \quad n$-ésima raiz da equação transcendental $\operatorname{com} c=0\left[\mathrm{~m}^{-1}\right]$

$\beta_{n, \infty} \quad n$-ésima raiz da equação transcendental $\operatorname{com} c \rightarrow \infty\left[\mathrm{m}^{-1}\right]$

$\gamma \quad$ Autovalor $\left[\mathrm{m}^{-1}\right]$

$\delta \quad$ Delta de Dirac

$\varepsilon \quad$ Emissividade superficial

$\zeta \quad$ Autovalor $\left[\mathrm{m}^{-1}\right]$

$\eta \quad$ Autovalor $\left[\mathrm{m}^{-1}\right]$

$\theta \quad$ Temperatura relativa $\left[{ }^{\circ} \mathrm{C}\right]$ ou $[\mathrm{K}]$ 
$\lambda$ Constante relacionada à separação de variáveis $\left[\mathrm{m}^{-2}\right]$

$\rho \quad$ Densidade $\left[\mathrm{kg} / \mathrm{m}^{3}\right]$

$\sigma \quad$ Constante de Stefan -Boltzmann $\left[\mathrm{W} / \mathrm{m}^{2} \cdot \mathrm{K}^{4}\right]$

$\psi \quad$ Temperatura em problema auxiliar $\left[{ }^{\circ} \mathrm{C}\right]$ ou $[\mathrm{K}]$

$\Gamma(\mathrm{t}) \quad$ Variável separada de $T$

Sobrescritos

* Variável adimensional

\section{Subscritos}

$\begin{array}{ll}i & \text { Inicial } \\ 0 & c=0 \\ \infty & c \rightarrow \infty\end{array}$




\section{SUMÁRIO}

Agradecimentos ........................................................................................................................... vii

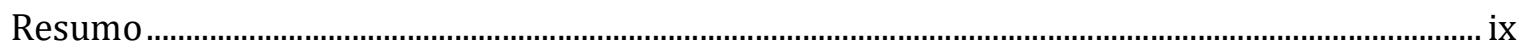

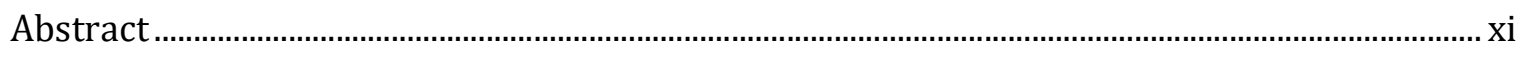

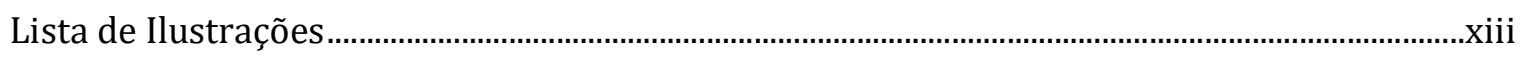

Lista de Tabelas.......................................................................................................................... Xvii

Lista de Símbolos............................................................................................................................. Xix

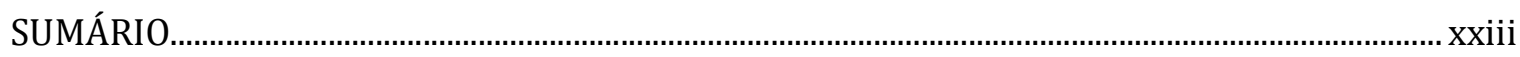

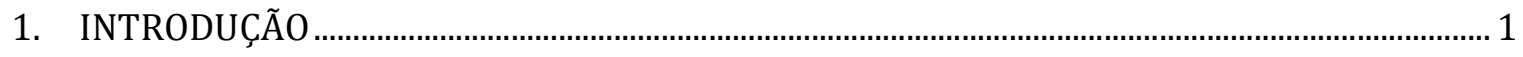

1.1. Os Dois Casos Simples de Condução de Calor ………....................................................... 1

1.2. Os Casos com Apenas Uma Não Homogeneidade ............................................................... 3

1.3. Os Casos com Mais de Uma Não Homogeneidade .............................................................10

1.4. Autovalores Sem Expressão em Forma Fechada ...............................................................11

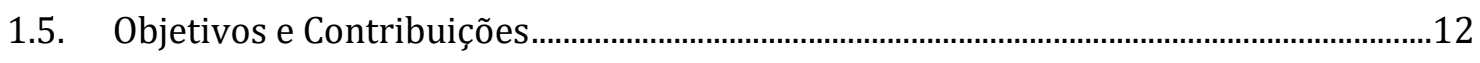

1.6. Observações Finais....................................................................................................

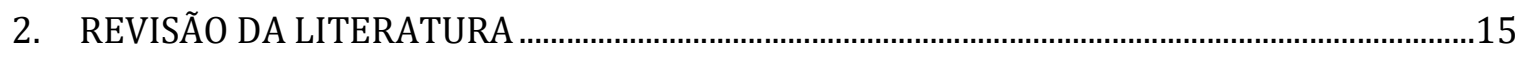

2.1. Expressões Iterativas para as Raízes das Eqs. (1.5), (1.6) e (1.7)..................................16

2.2. Propriedades das Raízes das Eqs. (1.5), (1.6) e (1.7)........................................................18

2.3. Expressões Aproximadas para a Primeira Raiz das Eqs. (1.5), (1.6) e (1.7) ...............24

2.4. Expressões Aproximadas para as Demais Raízes das Eqs. (1.5), (1.6) e (1.7).............34

3. NOVAS EXPRESSÕES ITERATIVAS PARA AS RAÍZES DAS EQS. (1.5), (1.6) E (1.7) ............39

3.1. Funções Iterativas obtidas com o Polinômio de Taylor .......................................................39

3.2. Funções Iterativas Com Derivadas Segundas..................................................................... 40

3.3. Funções Iterativas Com Derivadas Terceiras.......................................................................42

3.4. Funções Iterativas Com Derivadas de Ordem Maior do que Três .....................................44

3.5. Organização das Funções Iterativas.............................................................................45 
3.6. Comparação de Resultados ..................................................................................................

3.7. Aplicação para as Raízes das Eqs. (1.5), (1.6) e (1.7).......................................................48

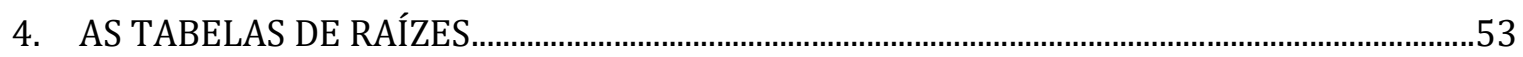

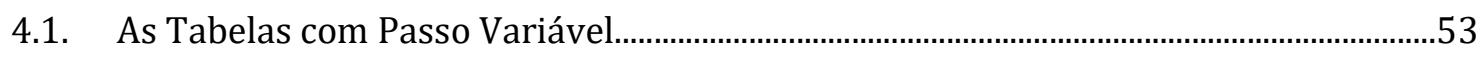

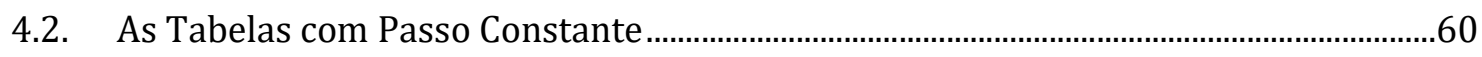

5. NOVAS EXPRESSÕES APROXIMADAS PARA A PRIMEIRA RAIZ ...............................................66

5.1. Uma Expressão que Utiliza Ponto Fixo ...............................................................................66

5.2. Coeficientes Ótimos para as Expressões Já Desenvolvidas .............................................68

5.3. Uma Expressão Algébrica ..................................................................................................75

6. NOVAS EXPRESSÕES APROXIMADAS PARA A PRIMEIRA RAIZ OBTIDAS A PARTIR DA

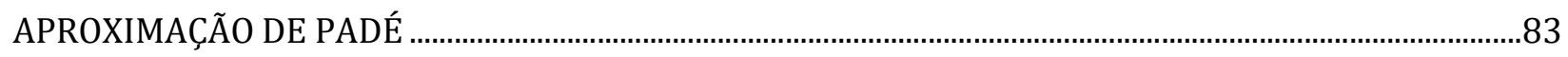

6.1. Expressões Geradas por Sistemas Algébricos Computacionais.......................................83

6.2. A Expansão em Frações Contínuas e a Aproximação de Padé..........................................89

6.3. Uma Expressão Obtida a Partir da Aproximação de Padé de Ordem $[1,1]$.................92

6.4. Uma Expressão Obtida a Partir da Aproximação de Padé de Ordem [1, 2] .................93

6.5. Uma Expressão Obtida a Partir da Aproximação de Padé de Ordem [2, 1] f................94

6.6. Uma Expressão Obtida a Partir da Aproximação de Padé de Ordem [2, 2] .................95

7. NOVAS EXPRESSÕES APROXIMADAS PARA AS DEMAIS RAÍZES ............................................98

7.1. A Generalização da Expressão Obtida a Partir da Aproximação de Padé de Ordem [1, 1] 98

7.2. A Generalização da Expressão de Yovanovich MiniMax 101

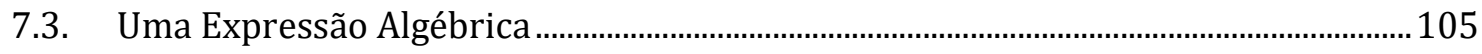

7.4. A Generalização da Expressão que Utiliza Ponto Fixo.......................................................106

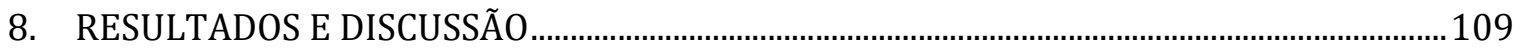

8.1. Expressões de Muito Menor Exatidão para a Primeira Raiz .........................................110

8.2. Expressões de Menor Exatidão para a Primeira Raiz ………….....................................112

8.3. Expressões de Intermediária Exatidão para a Primeira Raiz ........................................115 
8.4. Expressões de Maior Exatidão para a Primeira Raiz..............................................................117

8.5. Expressões de Muito Maior Exatidão para a Primeira Raiz ……………………………...119

8.6. Uma Expressão de Extrema Exatidão para a Primeira Raiz ...........................................121

8.7. Expressões de Menor Exatidão para as Demais Raízes.................................................. 122

8.8. Expressões de Maior Exatidão para as Demais Raízes ....................................................127

9. CONCLUSÕES E SUGESTÕES PARA PRÓXIMOS TRABALHOS …….......................................... 132

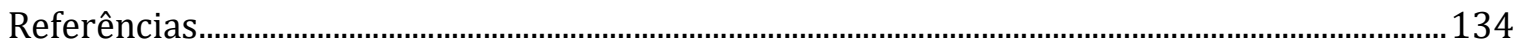

APÊNDICE A - O Método da Separação de Variáveis ............................................................................138

APÊNDICE B - O Método de Superposição ……................................................................................142

APÊNDICE C - A Função de Green.............................................................................................146 


\section{INTRODUÇÃO}

Em condução de calor, a temperatura no meio estacionário (que pode ser um sólido ou um fluido) pode variar com o tempo e com a posição. Serão apresentadas brevemente, na primeira seção deste capítulo, as duas situações mais simples nas quais a temperatura é função de apenas uma variável independente, para então se discutir as demais situações e o problema que surge quando ocorrem condições de contorno convectivas.

\subsection{Os Dois Casos Simples de Condução de Calor}

Há aplicações de engenharia nas quais a variação espacial da temperatura no interior do meio pode ser negligenciada, e a temperatura é considerada como função apenas do tempo. Essa abordagem proporciona uma grande simplificação e é conhecida como método da capacitância global.

Considere-se um meio estacionário com volume $V$, feito de um material com densidade $\rho$ e calor específico $c$, que no instante $t_{i}$ está a uma temperatura $T_{i}$, e começa a transferir calor para um ambiente com temperatura $T_{\infty}$ com um coeficiente de convecção $h$, e por radiação para uma vizinhança com temperatura $T_{v i z}$ e emissividade superficial $\varepsilon$, além de receber um fluxo de calor $q^{\prime \prime} \mathrm{e}$ possuir geração de energia interna por unidade de volume $q^{\prime \prime \prime}$. Denotando por $A_{s, c}$ a área da superfície que transfere calor por convecção, por $A_{s, r}$ a área da superfície que transfere calor por radiação, e por $A_{s, a}$ a área da superfície sobre a qual é aplicada fluxo de calor, um balanço da energia térmica deste meio leva diretamente a:

$$
q^{\prime \prime} A_{s, a}-h A_{s, c}\left(T-T_{\infty}\right)-\varepsilon \sigma A_{s, r}\left(T^{4}-T_{v i z}^{4}\right)+q^{\prime \prime \prime} V=\rho V c \frac{d T}{d t}
$$

que é uma equação diferencial ordinária separável e, então, pode ser resolvida com uma simples integração:

$$
\int_{T_{i}}^{T(t)} \frac{d T}{q^{\prime \prime} A_{s, a}-h A_{s, c}\left(T-T_{\infty}\right)-\varepsilon \sigma A_{s, r}\left(T^{4}-T_{v i z}^{4}\right)+q^{\prime \prime \prime} V}=\frac{t-t_{i}}{\rho V c}
$$

Esta integral não necessita de solução numérica em vários casos particulares, inclusive no caso de haver transferência de calor do meio apenas por radiação, e até mesmo no caso geral, se for utilizada a linearização:

$$
T^{4}-T_{v i z}^{4}=\left(T_{i}^{2}+T_{v i z}^{2}\right)\left(T_{i}+T_{v i z}\right)\left(T-T_{v i z}\right)
$$


No caso de não haver transferência de calor do meio por radiação, e $q^{\prime \prime}=q^{\prime \prime \prime}=0$, a solução é direta e sempre apresentada em textos de transferência de calor:

$$
\frac{T(t)-T_{\infty}}{T_{i}-T_{\infty}}=e^{-\frac{h A_{s, c}}{\rho V c}\left(t-t_{i}\right)}=e^{-B i \cdot F o}
$$

onde

$$
B i=\frac{h L_{c}}{k}
$$

é chamado de número de Biot,

$$
F o=\frac{k}{\rho c} \frac{\Delta t}{L_{c}^{2}}=\frac{\alpha \Delta t}{L_{c}^{2}}
$$

é um tempo adimensional chamado de número de Fourier, e

$$
L_{c}=\frac{V}{A_{s}}
$$

é chamado de comprimento característico.

Soluções exatas para a condução de calor através de sólidos na forma de parede, cilindro ou esfera, sujeitos a resfriamento convectivo mostram que para $B i<0,1$, a variação de temperatura dentro do sólido é menor do que 5\% (HAHN; ÖZIŞIK, 2012).

Há também aplicações de engenharia nas quais a condução de calor no meio estacionário ocorre em condições aproximadamente unidimensionais e em regime estacionário, ou seja, a temperatura depende apenas de uma coordenada espacial.

Considere-se um meio estacionário onde a condução de calor $q_{s}^{\prime \prime}$ em seu interior ocorre numa direção com coordenada $s$, ao mesmo tempo em que ocorre transferência de calor entre esse meio e seu ambiente e sua vizinhança, além do meio receber um fluxo de calor e possuir geração de energia interna. Através de duas seções transversais perpendiculares à direção da condução de calor e passando pelos pontos $s$ e $s+d s$, obtém-se um elemento sobre o qual um balanço de energia térmica leva a:

$$
\left(q^{\prime \prime}-h\left(T-T_{\infty}\right)-\sigma \varepsilon\left(T^{4}-T_{v i z}^{4}\right)\right)\left(d A_{\text {sup }}\right)+q^{\prime \prime \prime} A_{t r} d s+q_{s}^{\prime \prime}-\left(q_{s}^{\prime \prime}+\frac{d q_{s}^{\prime \prime}}{d s}\right)=0
$$

onde $A_{t r}$ é a área da seção transversal e $A_{\text {sup }}$ é a área da superfície. Utilizando a lei de Fourier:

$$
q_{s}^{\prime \prime}=-k A_{t r} \frac{d T}{d s}
$$

obtém-se

$$
k A_{t r} \frac{d^{2} T}{d s^{2}}+\frac{d}{d s}\left(k A_{t r}\right) \frac{d T}{d s}+\left(q_{s}^{\prime \prime}-h\left(T-T_{\infty}\right)-\sigma \varepsilon\left(T^{4}-T_{v i z}^{4}\right)\right) \frac{d A_{s u p}}{d s}+q^{\prime \prime \prime} A_{t r}=0
$$


que é uma equação diferencial ordinária que não necessita de solução numérica em vários casos particulares.

No caso de não haver transferência de calor através da superfície de uma camada plana, cilíndrica ou esférica de um meio, esta equação se simplifica para:

$$
k A_{t r} \frac{d^{2} T}{d s^{2}}+\frac{d}{d s}\left(k A_{t r}\right) \frac{d T}{d s}+q^{\prime \prime \prime} A_{t r}=0
$$

No caso de parede plana, $A_{t r}$ é constante, e $s=x$, então:

$$
\frac{d}{d x}\left(k \frac{d T}{d x}\right)+q^{\prime \prime \prime}=0
$$

no caso de parede cilíndrica, $A_{t r}=2 \pi r L$ e $s=r$, então:

$$
\frac{1}{r} \frac{d}{d r}\left(k r \frac{d T}{d r}\right)+q^{\prime \prime \prime}=0
$$

e, no caso de parede esférica, $A_{t r}=4 \pi r^{2}$ e $s=r$, então:

$$
\frac{1}{r^{2}} \frac{d}{d r}\left(k r^{2} \frac{d T}{d r}\right)+q^{\prime \prime \prime}=0
$$

as quais podem ser resolvidas facilmente e levam a constantes arbitrárias que podem ser determinadas através das condições de contorno. A partir destas soluções se definem resistências térmicas e se desenvolve boa parte da condução de calor elementar. As superfícies estendidas também são casos particulares desta equação diferencial:

$$
k A_{t r} \frac{d^{2} T}{d s^{2}}+\frac{d}{d s}\left(k A_{t r}\right) \frac{d T}{d s}-h\left(T-T_{\infty}\right) \frac{d A_{s u p}}{d s}=0
$$

que tem soluções que dependem muito da variação de $A_{t r}$ e $A_{\text {sup }}$ com $s$.

Enfim, nesses dois casos mais simples de condução de calor, onde a temperatura varia apenas com o tempo, $T=T(t)$, ou varia apenas com uma coordenada espacial, $T=T(s)$, as equações diferenciais resultantes são ordinárias. Para os demais casos, $T=T(x, y), T=T(r, \theta), T=$ $T(x, y, z, t)$, etc., e a equações diferenciais são parciais, sendo obtidas como demonstrado a seguir.

\subsection{Os Casos com Apenas Uma Não Homogeneidade}

Um balanço de energia térmica num elemento infinitesimal interno ao meio leva a:

$$
\int_{V} \rho c \frac{\partial T}{\partial t} d V=\int_{A} \boldsymbol{q}^{\prime \prime} \cdot d \boldsymbol{A}+\int_{V} q^{\prime \prime \prime} d V
$$

onde se aplica o teorema da divergência

$$
\int_{A} \boldsymbol{q}^{\prime \prime} \cdot d \boldsymbol{A}=\int_{V} \boldsymbol{\nabla} \cdot \boldsymbol{q}^{\prime \prime} d V
$$


obtendo-se

$$
\int_{V}\left(\rho c \frac{\partial T}{\partial t}-\nabla \cdot \boldsymbol{q}^{\prime \prime}+q^{\prime \prime \prime}\right) d V=0
$$

ou

$$
\rho c \frac{\partial T}{\partial t}-\nabla \cdot \boldsymbol{q}^{\prime \prime}+q^{\prime \prime \prime}=0
$$

Então, utilizando-se a Lei de Fourier:

$$
\boldsymbol{q}^{\prime \prime}=-k \nabla T
$$

obtém-se a equação do calor:

$$
\nabla \cdot(k \nabla T)+q^{\prime \prime \prime}=\rho c \frac{\partial T}{\partial t}
$$

onde, em coordenadas retangulares:

$$
\begin{gathered}
\vec{\nabla}=\hat{\imath} \frac{\partial}{\partial x}+\hat{\jmath} \frac{\partial}{\partial y}+\hat{k} \frac{\partial}{\partial z} \rightarrow \\
\frac{\partial}{\partial x}\left(k \frac{\partial T}{\partial x}\right)+\frac{\partial}{\partial y}\left(k \frac{\partial T}{\partial y}\right)+\frac{\partial}{\partial z}\left(k \frac{\partial T}{\partial z}\right)+q^{\prime \prime \prime}=\rho c \frac{\partial T}{\partial t}
\end{gathered}
$$

em coordenadas cilíndricas:

$$
\begin{gathered}
\vec{\nabla}=\hat{e}_{r} \frac{\partial}{\partial r}+\hat{e}_{\phi} \frac{1}{r} \frac{\partial}{\partial \phi}+\hat{k} \frac{\partial}{\partial z} \rightarrow \\
\frac{1}{r} \frac{\partial}{\partial r}\left(k r \frac{\partial T}{\partial r}\right)+\frac{1}{r^{2}} \frac{\partial}{\partial \phi}\left(k \frac{\partial T}{\partial \phi}\right)+\frac{\partial}{\partial z}\left(k \frac{\partial T}{\partial z}\right)+q^{\prime \prime \prime}=\rho c \frac{\partial T}{\partial t}
\end{gathered}
$$

e em coordenadas esféricas:

$$
\begin{gathered}
\vec{\nabla}=\hat{e}_{r} \frac{\partial}{\partial r}+\hat{e}_{\theta} \frac{1}{r} \frac{\partial}{\partial \theta}+\hat{e}_{\phi} \frac{1}{r \sin \theta} \frac{\partial}{\partial \phi} \rightarrow \\
\frac{1}{r^{2}} \frac{\partial}{\partial r}\left(k r^{2} \frac{\partial T}{\partial r}\right)+\frac{1}{r^{2} \sin ^{2} \theta} \frac{\partial}{\partial \phi}\left(k \frac{\partial T}{\partial \phi}\right)+\frac{1}{r^{2} \sin \theta} \frac{\partial}{\partial \theta}\left(k \sin \theta \frac{\partial T}{\partial \theta}\right)+q^{\prime \prime \prime}=\rho c \frac{\partial T}{\partial t}
\end{gathered}
$$

No caso mais comum de condutividade térmica constante, a equação do calor simplifica-se para:

$$
\nabla^{2} T+\frac{q^{\prime \prime \prime}}{k}=\frac{1}{\alpha} \frac{\partial T}{\partial t}
$$

e as formas acima, para cada sistema de coordenadas, são analogamente simplificadas.

Considere-se o problema de valor de contorno que corresponde à condução de calor em regime permanente através de um meio com forma de paralelepípedo, com duas superfícies paralelas de dimensões bem menores do que as quatro outras, ou isoladas, de maneira que o calor transferido por elas possa ser negligenciado. Destas quatro outras faces, supõe-se que duas delas (não paralelas) tenham temperaturas mantidas a $0^{\circ} \mathrm{C}$, enquanto que uma das outras duas tenha sua temperatura 
mantida a valores conhecidos e fixos, e a última esteja transferindo calor por convecção para um ambiente a $0^{\circ} \mathrm{C}$ (Figura 1.1).

Então, posicionando adequadamente o sistema de coordenadas, a equação do calor e as condições de contorno resultam em:

$$
\left\{\begin{array}{l}
\frac{\partial^{2} T}{\partial x^{2}}+\frac{\partial^{2} T}{\partial y^{2}}=0, \quad 0<x<L, 0<y<W \\
T(0, y)=0, \quad 0<y<W, \quad T=0 \\
\frac{\partial T}{\partial x}(L, y)+H T(L, y)=0, \quad 0<y<W \\
T(x, 0)=0, \quad 0<x<L \\
T(x, W)=f(x), \quad 0<x<L
\end{array}\right.
$$

onde $H$ é a razão entre o coeficiente de convecção e a condutividade térmica do meio.

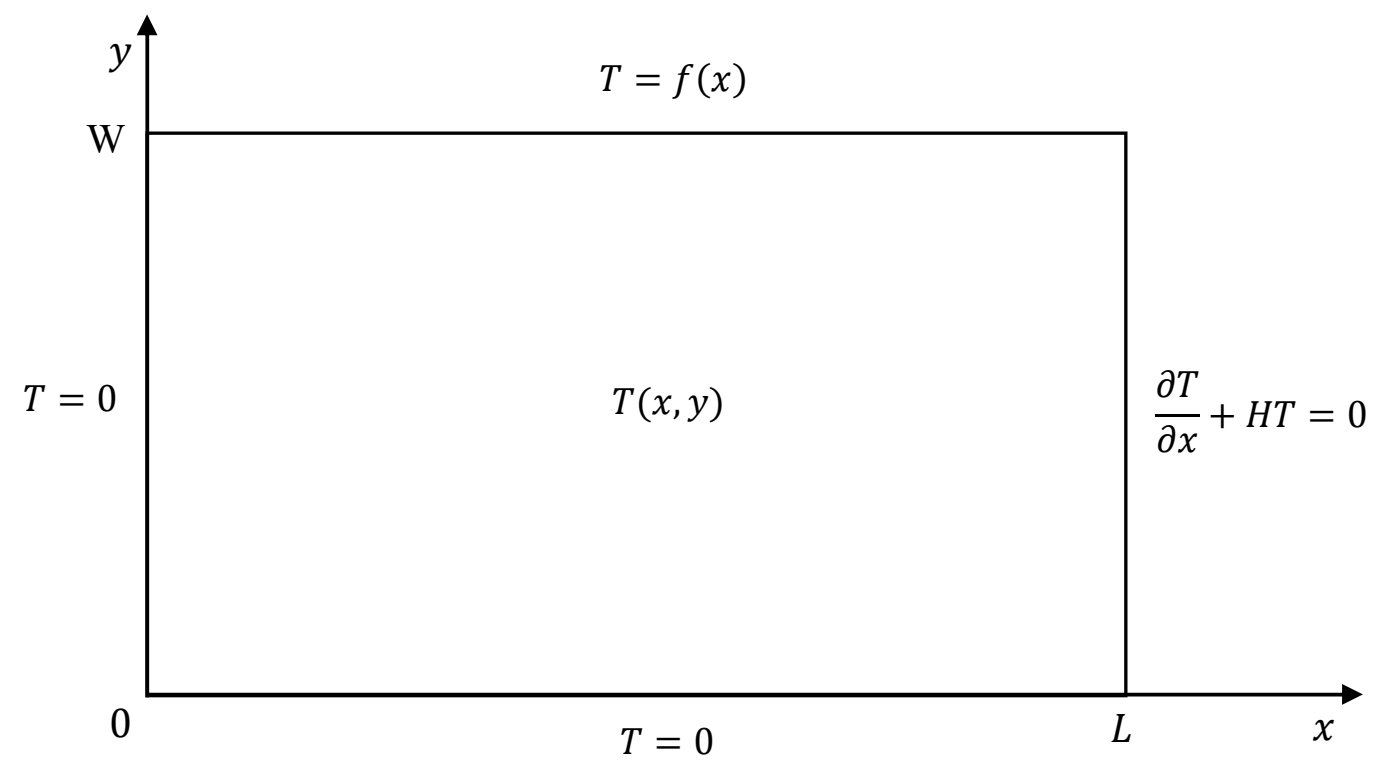

Figura 1.1 Vista de paralelepípedo com a face frontal correspondente a uma das superfícies com dimensões pequenas ou isoladas, e com face esquerda com temperatura nula

Através do método de separação de variáveis obtém-se que a solução desse problema é:

$$
T(x, y)=\sum_{n}\left(\frac{1}{L \operatorname{senh} \zeta_{n} W}\left(\int_{-L}^{L} f(x) \operatorname{sen} \zeta_{n} x d x\right) \operatorname{sen} \zeta_{n} x \operatorname{senh} \zeta_{n} y\right)
$$

onde os autovalores $\zeta_{n}$ são as raízes de:

$$
\zeta_{n} \cos \zeta_{n} L+H \operatorname{sen} \zeta_{n} L=0
$$

que não possui solução que possa ser expressa em forma fechada. 
Se uma das paredes que está com temperatura fixada em $0^{\circ} \mathrm{C}$, estivesse, em vez disso, isolada (Figura 1.2), o problema passaria a ser:

$$
\left\{\begin{array}{l}
\frac{\partial^{2} T}{\partial x^{2}}+\frac{\partial^{2} T}{\partial y^{2}}=0, \quad 0<x<L, 0<y<W \\
\frac{\partial T}{\partial x}(0, y)=0, \quad 0<y<W \\
\frac{\partial T}{\partial x}(L, y)+H T(L, y)=0, \quad 0<y<W \\
T(x, 0)=0, \quad 0<x<L \\
T(x, W)=f(x), \quad 0<x<L
\end{array}\right.
$$

onde os autovalores $\zeta_{n}$ são as raízes de:

$$
\zeta_{n} \operatorname{sen} \zeta_{n} L-H \cos \zeta_{n} L=0
$$

que também não possui solução que possa ser expressa em forma fechada.

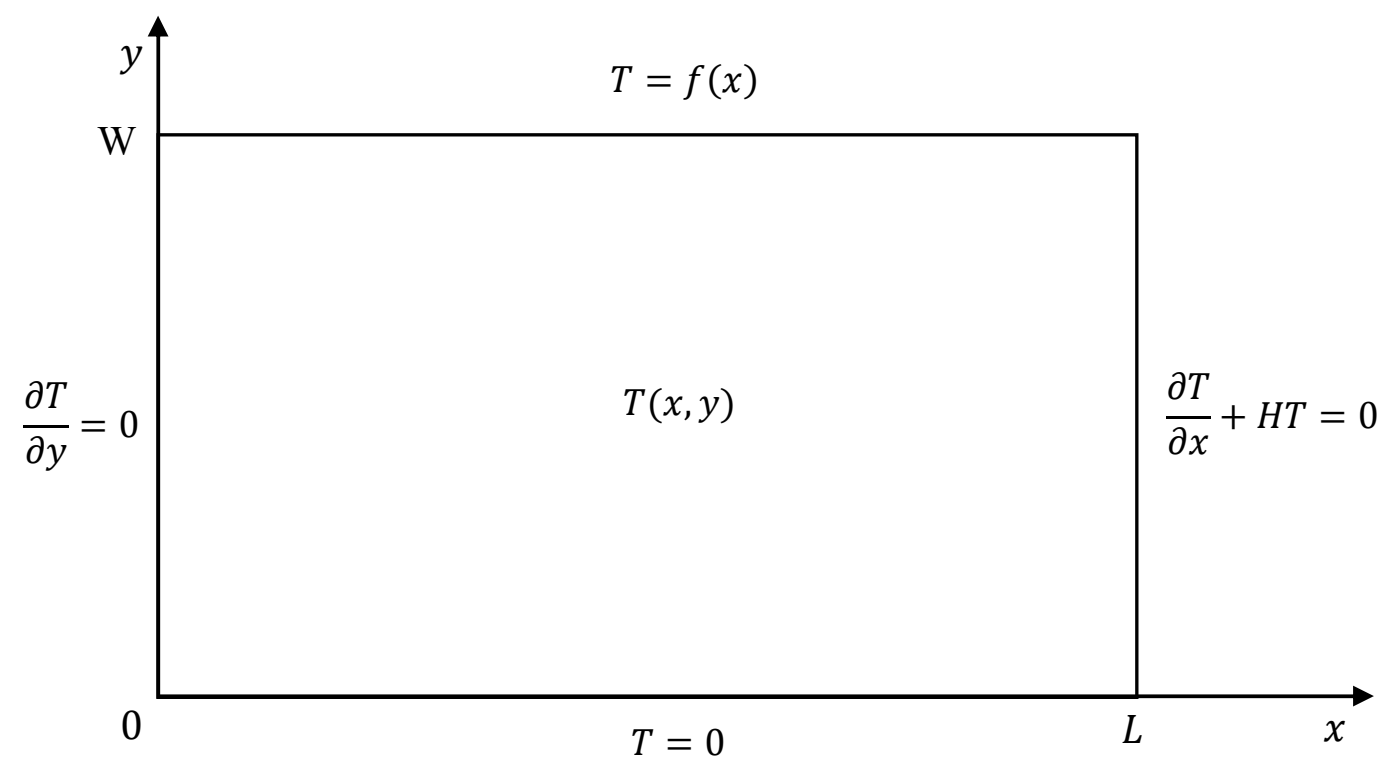

Figura 1.2 Vista de paralelepípedo com a face frontal correspondente a uma das superfícies com dimensões pequenas ou isoladas, e com face esquerda isolada

A aplicação desse método está detalhada no Apêndice A para este exemplo, mas não está detalhada para os três próximos exemplos, por serem muito análogos a este. Apenas observe-se que alguns textos elementares de transferência de calor associam a Eq. (1.2) ao problema unidimensional transitório em coordenadas retangulares, e a Eq. (1.1) ao problema unidimensional transitório em 
coordenadas cilíndricas. Porém, estas duas equações podem aparecer em mais problemas, como esses dois problemas bidimensionais permanentes mostrados.

Considere-se o problema de valor de contorno que corresponde à condução de calor em regime transitório através de um meio com forma de paralelepípedo, com quatro superfícies paralelas duas a duas, bem menores do que as outras duas, ou isoladas, de maneira que o calor transferido por elas possa ser negligenciado. Supõe-se que o meio tenha temperatura inicial conhecida e dependente apenas da distância em relação às faces maiores, sendo que uma delas tem temperatura mantida a $0^{\circ}$ $\mathrm{C}$, enquanto que a outra esteja transferindo calor por convecção para um ambiente também a $0^{\circ} \mathrm{C}$ (Figura 1.3).

Então:

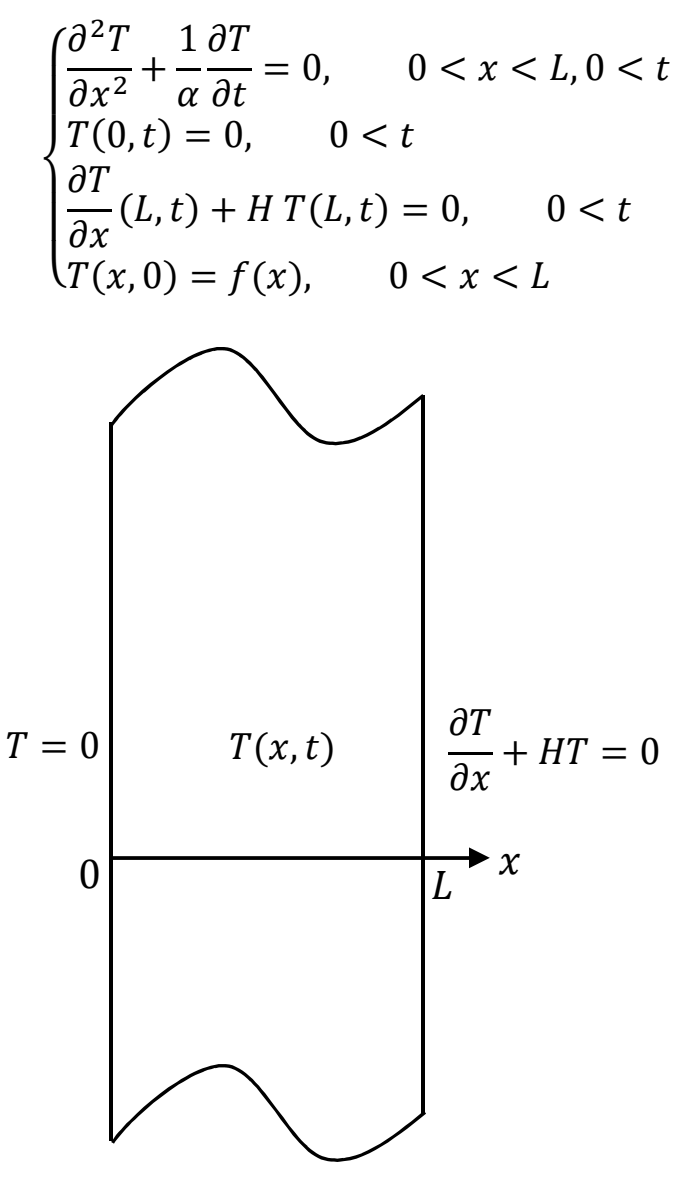

Figura 1.3 Vista de paralelepípedo com a face frontal correspondente a uma das superfícies com dimensões maiores ou não isoladas

Através de separação de variáveis, obtém-se que a solução desse problema é:

$$
T(x, t)=\sum_{n}\left(\frac{1}{L}\left(\int_{-L}^{L} f(x) \operatorname{sen} \frac{n \pi x}{L} d x\right) \operatorname{sen} \zeta_{n} x \exp \zeta_{n}^{2} t\right)
$$


onde os autovalores $\zeta_{n}$ são as raízes da Eq. (1.2). Caso a parede que está com temperatura de $0^{\circ} \mathrm{C}$ estivesse isolada, obter-se-ia, da mesma maneira, que os autovalores são as raízes da Eq. (1.1).

Considere-se o problema de valor de contorno que corresponde à condução de calor em regime permanente através de um meio com forma de cilindro, com a área das bases bem menor do que a área lateral, o qual está transferindo calor por convecção para um ambiente a $0^{\circ} \mathrm{C}$ enquanto que uma das bases tem temperatura prescrita com simetria circular (Figura 1.4).

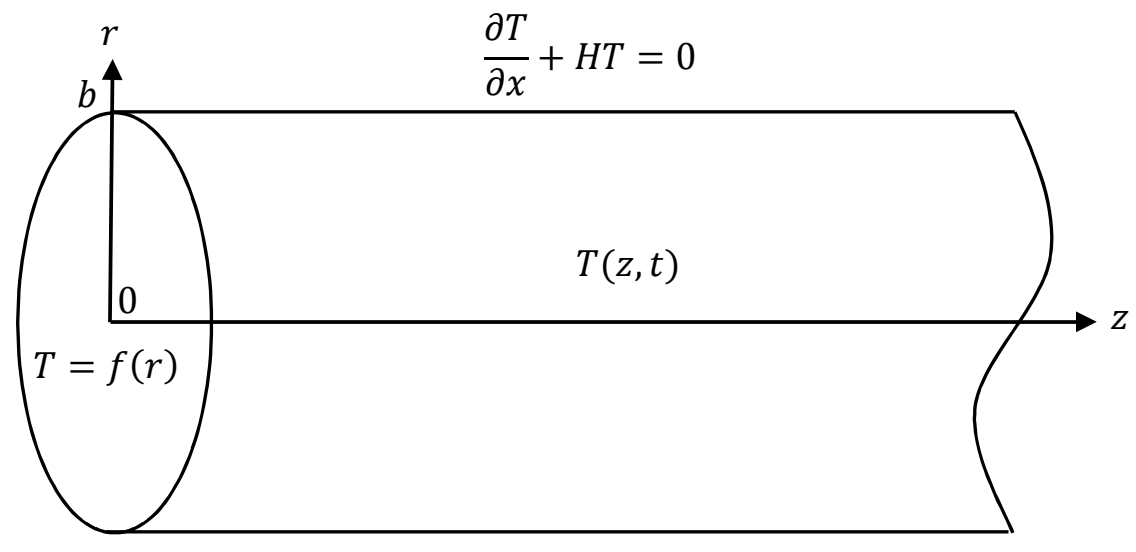

Figura 1.4 Cilindro com bases bem menores do que a superfície lateral

Então:

$$
\left\{\begin{array}{l}
\frac{1}{r} \frac{\partial}{\partial r}\left(r \frac{\partial T}{\partial r}\right)+\frac{\partial^{2} T}{\partial z^{2}}=0, \quad 0 \leq r<b, 0<z \\
T(0, z)=\text { finita, } \quad 0<z \\
\frac{\partial T}{\partial r}(b, z)+H T(b, z)=0, \quad 0<z \\
T(r, 0)=f(r), \quad 0<r<b \\
T(r, \infty)=0, \quad 0<r<b
\end{array}\right.
$$

Através de separação de variáveis, obtém-se que a solução desse problema é:

$$
T(r, z)=\sum_{n}\left(\frac{2}{b^{2} \mathrm{~J}_{1}^{2}\left(\zeta_{n} r\right)}\left(\int_{0}^{b} f(r) \mathrm{J}_{0}\left(\zeta_{n} r\right) d r\right) \mathrm{J}_{0}\left(\zeta_{n} r\right) \exp \left(-\zeta_{n} z\right)\right)
$$

onde os autovalores $\zeta_{n}$ são as raízes de:

$$
\zeta_{n} \mathrm{~J}_{0}^{\prime}\left(\zeta_{n} b\right)+H \mathrm{~J}_{0}\left(\zeta_{n} b\right)=0
$$

que também não possui solução que possa ser expressa em forma fechada.

Considere-se o problema de valor de contorno que corresponde à condução de calor em regime transitório através de um meio com forma de esfera, com temperatura inicial conhecida e com 
simetria esférica, e cuja superfície esteja transferindo calor por convecção para um ambiente a $0^{\circ} \mathrm{C}$ (Figura 1.5).

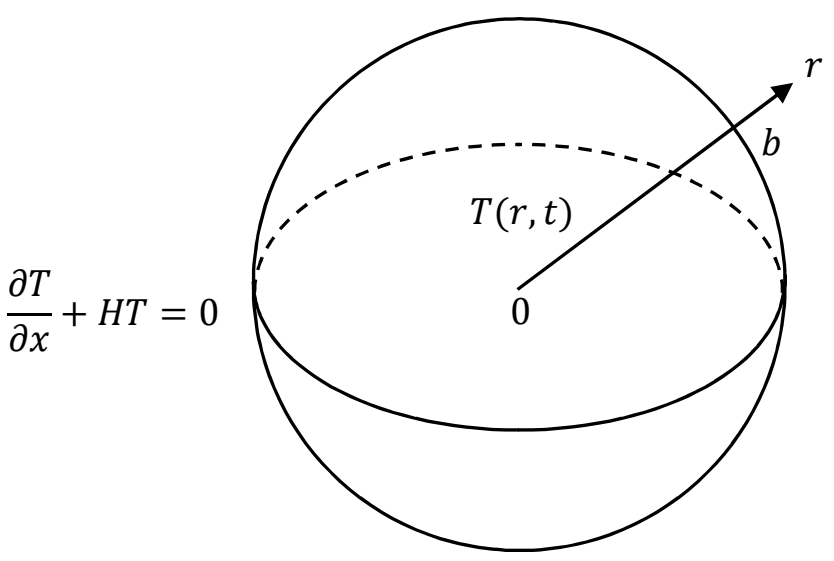

Figura 1.5 Esfera transferindo calor

Então:

$$
\left\{\begin{array}{l}
\frac{1}{r^{2}} \frac{\partial}{\partial r}\left(r^{2} \frac{\partial T}{\partial r}\right)+\frac{1}{\alpha} \frac{\partial T}{\partial t}=0, \quad 0 \leq r<b, 0<t \\
T(0, t)=\text { finita, } \quad 0<t \\
\frac{\partial T}{\partial r}(b, t)+H T(b, t)=0, \quad 0<t \\
T(r, 0)=f(r), \quad 0<r<b
\end{array}\right.
$$

Através de separação de variáveis, obtém-se que a solução desse problema é:

$$
T(r, t)=\frac{1}{r} \sum_{n}\left(\frac{2}{L}\left(\int_{0}^{b} r f(r) \operatorname{sen} \zeta_{n} r d r\right) \operatorname{sen} \zeta_{n} r \exp \zeta_{n}^{2} t\right)
$$

e os autovalores $\zeta_{n}$ são dados por

$$
\zeta_{n} \cos \zeta_{n} b+\left(H-\frac{1}{b}\right) \operatorname{sen} \zeta_{n} b=0
$$

que também não possui solução que possa ser expressa em forma fechada.

Os quatro exemplos apresentados acima contêm equações diferenciais e condições de contorno e iniciais homogêneas, com exceção de uma dessas condições para cada problema. Será mostrado agora que as mesmas equações dos autovalores surgem em problemas mais gerais. 


\subsection{Os Casos com Mais de Uma Não Homogeneidade}

Considere-se o problema com equação diferencial e condições de contorno e inicial todas não homogêneas:

$$
\left\{\begin{array}{l}
\frac{\partial^{2} T}{\partial x^{2}}+\frac{\partial^{2} T}{\partial y^{2}}+\frac{q^{\prime \prime \prime}(x, y)}{k}=\frac{1}{\alpha} \frac{\partial T}{\partial t}, \quad 0<x<L_{1}, 0<y<L_{2}, 0<t \\
-k_{1} \frac{\partial T}{\partial x}(0, y, t)+h_{1} T(0, y, t)=f_{1}(y), \quad 0<y<L_{2}, 0<t \\
k_{2} \frac{\partial T}{\partial x}\left(L_{1}, y, t\right)+h_{2} T\left(L_{1}, y, t\right)=f_{2}(y), \quad 0<y<L_{2}, 0<t \\
-k_{3} \frac{\partial T}{\partial y}(x, 0, t)+h_{3} T(x, 0, t)=f_{3}(x), \quad 0<x<L_{1}, 0<t \\
k_{4} \frac{\partial T}{\partial y}\left(x, L_{2}, t\right)+h_{4} T\left(x, L_{2}, t\right)=f_{4}(x), \quad 0<x<L_{1}, 0<t \\
T(x, y, 0)=f_{5}(x, y), \quad 0<x<L_{1}, 0<y<L_{2}
\end{array}\right.
$$

Observe-se que essas condições de contorno generalizam os três tipos possíveis, porque se for feito $k_{i}=0$ e $h_{i}=h$, tem-se uma condição do primeiro tipo, se for feito $k_{i}=k$ e $h_{i}=0$, tem-se uma condição do segundo tipo, e se for feito $k_{i}=k$ e $h_{i}=h$, tem-se uma condição do terceiro tipo. A solução desse problema através de superposição está mostrada no Apêndice B.

O caso 3D é uma extensão muito direta e que ocuparia muito espaço para ser mostrado. Os casos análogos em coordenadas cilíndricas e esféricas podem ser tratados do mesmo modo e também não serão mostrados.

Um problema ainda mais geral, onde as condições de contorno e geração de energia dependem do tempo:

$$
\left\{\begin{array}{l}
\frac{\partial^{2} T}{\partial x^{2}}+\frac{\partial^{2} T}{\partial y^{2}}+\frac{\partial^{2} T}{\partial z^{2}}+\frac{q^{\prime \prime \prime}(x, y, z, t)}{k}=\frac{1}{\alpha} \frac{\partial T}{\partial t}, \quad 0<x<L_{1}, 0<y<L_{2}, 0<z<L_{3}, 0<t \\
-k_{1} \frac{\partial T}{\partial x}(0, y, z, t)+h_{1} T(0, y, z, t)=f_{1}(y, z, t), \quad 0<y<L_{2}, 0<z<L_{3}, 0<t \\
k_{2} \frac{\partial T}{\partial x}\left(L_{1}, y, z, t\right)+h_{2} T\left(L_{1}, y, z, t\right)=f_{2}(y, z, t), \quad 0<y<L_{2}, 0<z<L_{3}, 0<t \\
-k_{3} \frac{\partial T}{\partial y}(x, 0, z, t)+h_{3} T(x, 0, z, t)=f_{3}(x, z, t), \quad 0<x<L_{1}, 0<z<L_{3}, 0<t \\
k_{4} \frac{\partial T}{\partial y}\left(x, L_{2}, z, t\right)+h_{4} T\left(x, L_{2}, z, t\right)=f_{4}(x, z, t), \quad 0<x<L_{1}, 0<z<L_{3}, 0<t \\
-k_{5} \frac{\partial T}{\partial z}(x, y, 0, t)+h_{5} T(x, y, 0, t)=f_{5}(x, y, t), \quad 0<x<L_{1}, 0<y<L_{2}, 0<t \\
k_{6} \frac{\partial T}{\partial z}\left(x, y, L_{3}, t\right)+h_{6} T\left(x, y, L_{3}, t\right)=f_{6}(x, y, t), \quad 0<x<L_{1}, 0<y<L_{2}, 0<t \\
T(x, y, z, 0)=f_{7}(x, y, z), \quad 0<x<L_{1}, 0<y<L_{2}, 0<z<L_{3}
\end{array}\right.
$$


está resolvido através de funções de Green no Apêndice C, método este reconhecido como o mais geral existente para resolver problemas de condução de calor.

Observe-se que esses métodos mais avançados de solução, que permitem se abordar problemas mais elaborados, levam às mesmas equações de autovalores do que o método da separação de variáveis. Isso também ocorre com outros métodos, como a técnica da transformada integral, a transformada de Laplace, e o teorema de Duhamel.

\subsection{Autovalores Sem Expressão em Forma Fechada}

Hahn e Özişik (2012) apresentam as equações dos autovalores de problemas com várias condições de contorno e sistemas de coordenadas. As soluções em forma fechada são simplesmente as raízes de funções conhecidas:

$$
\left\{\begin{array}{l}
\sin \zeta_{n} L=0 \rightarrow \zeta_{n}=n \frac{\pi}{L}, n=1,2, \ldots \\
\cos \zeta_{n} L=0 \rightarrow \zeta_{n}=\left(n-\frac{1}{2}\right) \frac{\pi}{L}, n=1,2, \ldots \\
\mathrm{J}_{0}\left(\zeta_{n} b\right)=0 \rightarrow \zeta_{n}=\frac{2,4048}{b}, \frac{5,5201}{b}, \frac{8,6537}{b}, \ldots \\
\mathrm{J}_{1}\left(\zeta_{n} b\right)=0 \rightarrow \zeta_{n}=\frac{3,8317}{b}, \frac{7,0156}{b}, \frac{10,1735}{b}, \ldots
\end{array}\right.
$$

e as que não tem solução em forma fechada são:

$$
\left\{\begin{array}{l}
\zeta_{n} \operatorname{sen} \zeta_{n} L-H \cos \zeta_{n} L=0 \\
\zeta_{n} \mathrm{~J}_{0}^{\prime}\left(\zeta_{n} b\right)+H \mathrm{~J}_{0}\left(\zeta_{n} b\right)=0 \\
\zeta_{n} \cos \zeta_{n} L+H \operatorname{sen} \zeta_{n} L=0 \\
\zeta_{n} \cos \zeta_{n} b+\left(H-\frac{1}{b}\right) \operatorname{sen} \zeta_{n} b=0 \\
\mathrm{~J}_{v}^{\prime}\left(\zeta_{n} b\right)=0 \\
\beta_{n} \mathrm{~J}_{v}^{\prime}\left(\zeta_{n} b\right)+H \mathrm{~J}_{v}\left(\zeta_{n} b\right)=0 \\
\tan \zeta_{n} L=\frac{\zeta_{n}\left(H_{1}+H_{2}\right)}{\zeta_{n}^{2}-H_{1} H_{2}} \\
\mathrm{~J}_{v}\left(\zeta_{n} a\right) Y_{v}\left(\zeta_{n} b\right)-\mathrm{J}_{v}\left(\zeta_{n} b\right) Y_{v}\left(\zeta_{n} a\right)=0 \\
\mathrm{~J}_{v}^{\prime}\left(\zeta_{n} b\right) Y_{v}\left(\zeta_{n} a\right)-\mathrm{J}_{v}\left(\zeta_{n} a\right) Y_{v}^{\prime}\left(\zeta_{n} b\right)=0 \\
\mathrm{Y}_{v}^{\prime}\left(\zeta_{n} a\right)-\mathrm{J}_{v}^{\prime}\left(\zeta_{n} a\right) \mathrm{Y}_{v}\left(\zeta_{n} b\right)=0 \\
\mathrm{~J}_{v}^{\prime}\left(\zeta_{n} a\right) Y_{v}^{\prime}\left(\zeta_{n} b\right)-\mathrm{J}_{v}^{\prime}\left(\zeta_{n} b\right) Y_{v}^{\prime}\left(\zeta_{n} a\right)=0
\end{array}\right.
$$

As Eqs. (1.1) a (1.4) foram exemplificadas através de problemas físicos, e são as quatro primeiras desta lista. Elas são as mais utilizadas, são o foco deste trabalho e podem ser reescritas como: 


$$
\left\{\begin{array}{l}
\left(\zeta_{n} L\right) \tan \left(\zeta_{n} L\right)=B i \\
\left(\zeta_{n} b\right) \frac{\mathrm{J}_{1}\left(\zeta_{n} b\right)}{\mathrm{J}_{0}\left(\zeta_{n} b\right)}=B i \\
\left(\zeta_{n} L\right) \cot \left(\zeta_{n} L\right)=-B i \\
\left(\zeta_{n} b\right) \cot \left(\zeta_{n} b\right)=1-B i
\end{array}\right.
$$

onde $B i=H L=h L / k$ ou $B i=H b=h b / k$.

Estas equações também ocorrem em outras áreas da física e da engenharia (CRANK, 1975), como, por exemplo, no problema de vibração longitudinal em barras uniformes com um extremo fixo e outro com massa fixa. Então, em vez de se utilizar $B i$, se utilizará o símbolo $c$ que pode representar uma constante adimensional de qualquer área de conhecimento. Além disso, as últimas duas equações são equivalentes, e apenas uma delas precisa ser estudada. Assim, são escolhidas para este trabalho as seguintes formas para estas equações:

$$
\begin{gathered}
\beta_{n} \tan \beta_{n}=c \\
\beta_{n} \frac{\mathrm{J}_{1}\left(\beta_{n}\right)}{\mathrm{J}_{0}\left(\beta_{n}\right)}=c \\
\beta_{n} \cot \beta_{n}=1-c
\end{gathered}
$$

onde $\beta_{n}$ são as raízes a partir das quais se obtém os autovalores: $\zeta_{n}=\beta_{n} / L$ ou $\zeta_{n}=\beta_{n} / b$.

\subsection{Objetivos e Contribuições}

O objetivo desta tese é o de se encontrar expressões aproximadas e expressões iterativas para as raízes $\beta_{n}$ das Eqs. (1.5), (1.6) e (1.7), as quais surgem sempre que uma das condições de contorno envolve convecção (a menos que a única condição não homogênea seja justamente ela), ou seja, na maioria dos problemas de condução de calor com mais de uma variável independente e com condição de contorno convectiva, o que evidencia sua importância.

As contribuições deste trabalho serão:

- Desenvolvimento de uma nova família de funções iterativas para a busca de raízes;

- Demonstração de que uma dessas funções iterativas é a que tem convergência mais rápida para a busca das raízes das três equações transcendentes que são abordadas neste trabalho;

- Análise de erros das tabelas de raízes que estão disponíveis nos textos e manuais de transferência de calor; 
- Desenvolvimento de expressões aproximadas tanto para a primeira como para as demais raízes das três equações abordadas, com erros menores do que os das tabelas de raízes.

\subsection{Observações Finais}

Em alguns problemas adimensionalizados, as raízes são os próprios autovalores, ou seja, elas não precisam ser divididas por um comprimento ou por um raio para levarem aos autovalores. Por exemplo, uma parede com temperatura inicial uniforme, uma face isolada, e outra com convecção, é modelada por:

$$
\left\{\begin{array}{l}
\frac{\partial^{2} T}{\partial x^{2}}=\frac{1}{\alpha} \frac{\partial T}{\partial t} \\
T(x, 0)=T_{i} \\
\left.\frac{\partial T}{\partial x}\right|_{x=0}=0 \\
-\left.k \frac{\partial T}{\partial x}\right|_{x=L}=h\left(T(L, t)-T_{\infty}\right)
\end{array}\right.
$$

Utilizando as variáveis adimensionalizadas:

$$
\theta^{*} \equiv \frac{\theta}{\theta_{i}}=\frac{T-T_{\infty}}{T_{i}-T_{\infty}}, x^{*} \equiv \frac{x}{L}, t^{*} \equiv \frac{\alpha t}{L^{2}} \equiv F o
$$

a solução é apresentada em livros elementares de transferência de calor:

$$
\theta^{*}=\sum_{n=1}^{\infty} \frac{4 \operatorname{sen} \zeta_{n}}{2 \zeta_{n}+\operatorname{sen} 2 \zeta_{n}} e^{-\zeta_{n}^{2} x^{*}} \cos \zeta_{n} x^{*}
$$

onde os autovalores $\zeta_{n}$ são as próprias raízes $\beta_{n}$ da Eq. (1.5), ou seja, neste caso $\zeta_{n}=\beta_{n}$.

A Eq. (1.6) pode parecer muito diferente das outras duas, porém, isto ocorre apenas porque, apesar de

$$
\frac{\cos ^{\prime} \beta_{n} L}{\cos \beta_{n} L}=-\frac{\operatorname{sen} \beta_{n} L}{\cos \beta_{n} L}=-\tan \beta_{n} L
$$

$\mathrm{e}$

$$
\frac{\operatorname{sen}^{\prime} \beta_{n} L}{\operatorname{sen} \beta_{n} L}=\frac{\cos \beta_{n} L}{\operatorname{sen} \beta_{n} L}=\cot \beta_{n} L
$$

não existe uma definição análoga para as razões das funções de Bessel:

$$
\frac{\mathrm{J}_{0}^{\prime}\left(\beta_{n} b\right)}{\mathrm{J}_{0}\left(\beta_{n} b\right)}=-\frac{\mathrm{J}_{1}\left(\beta_{n} b\right)}{\mathrm{J}_{0}\left(\beta_{n} b\right)}
$$


Essa restrição não implica apenas que a forma da correspondente equação não seja compacta. Haverá limitações de alguns métodos que precisam inverter as funções tangente e cotangente e que não poderão fazer o mesmo com a razão entre as funções de Bessel.

Ao longo deste trabalho, chamaremos de "erro absoluto" o valor aproximado menos o valor exato, que pode, então, ser negativo. Isto justifica por que, em alguns momentos, faremos referência ao "módulo do erro absoluto". Por outro lado, chamaremos de "erro relativo" o erro absoluto dividido pelo valor exato e, então, poderemos nos referir também ao "módulo do erro relativo". 0 erro relativo será expresso, algumas vezes, em percentuais, quando, então, poderemos nos referir simplesmente a "erros".

Um fato de grande importância para o presente trabalho é o de que a exatidão da primeira raiz é a mais relevante. Pode-se calcular que para $F_{0}>0,2$, mantendo-se o primeiro termo e negligenciando-se todos os demais termos nas séries, resulta em erro relativo com módulo menor do que $2 \%$. Isto ocorre devido à convergência das séries que implica em rápida diminuição dos valores de um termo para outro. Também é importante observar que o aumento da exatidão de cálculo da primeira raiz é interessante para o caso em que as demais raízes também estão sendo calculadas. 


\section{REVISÃO DA LITERATURA}

Expressões para as raízes das Eqs. (1.5), (1.6) e (1.7), cuja obtenção é o objetivo deste trabalho, já foram desenvolvidas também por outros autores. Serão analisados estes trabalhos na ordem cronológica, dividindo as expressões por três seções, uma para as expressões iterativas, uma outra para as expressões aproximadas fechadas da primeira raiz e uma final para as expressões aproximadas fechadas das demais raízes.

Porém, antes de iniciar esta revisão, salienta-se que além das expressões iterativas e das expressões aproximadas, existem também expressões explícitas para estas raízes. Leathers e McCormick (1996) realizaram um levantamento das que são utilizadas na área de transferência de calor, e uma delas corresponde à Eq. (1.5), a qual foi obtida por Burniston e Siewert (1973) através da substituição $\beta_{n}=i \omega z$ :

$$
\beta_{n}=\left\{\begin{array}{l}
\left(\frac{\pi \omega}{2}\right)^{1 / 2} \exp \left(-\frac{1}{\pi} \int_{0}^{1}\left(\arg \Lambda_{0}^{+}(v)+\frac{\pi}{2}\right) \frac{d v}{v}\right), n=1 \\
\frac{\pi}{2}\left(4 n^{2}-8 n+3\right)^{1 / 2} \exp \left(-\frac{1}{\pi} \int_{0}^{1} \arg \Omega_{n}^{+}(v) \frac{d v}{v}\right), n=2,3, \ldots
\end{array}\right.
$$

onde

$$
\begin{gathered}
\Lambda_{0}^{+}(v)=v\left(v-\frac{1}{2 \omega} \ln \frac{1-v}{1+v}\right)-\frac{\pi i v}{2 \omega} \\
v^{2} \Omega_{n}^{+}(v)=\left(\Lambda_{0}^{+}(v)\right)^{2}+\frac{(n-1)^{2} \pi^{2} v^{2}}{\omega^{2}}
\end{gathered}
$$

Simplificamos a expressão da primeira raiz:

$$
\beta_{1}=\sqrt{\frac{\pi}{2} c} \exp \int_{0}^{1}\left(\frac{1}{\pi / 2} \arctan \frac{\pi / 2}{v c-\frac{1}{2} \ln \left(\frac{1-v}{1+v}\right)}-1\right) \frac{d v}{2 v}
$$

e confirmamos seus resultados através de integração numérica pelo método elementar dos trapézios. A singularidade no limite inferior que faz essa integral ser imprópria não causou dificuldade para sua convergência.

Luck e Stevens (2002) estenderam significativamente essa ideia apresentando um método baseado no teorema integral de Cauchy para se obter expressões explícitas para as raízes de qualquer equação transcendental. Contudo, expressões explícitas nem sempre são classificadas como fechadas (STOVER; WEISSTEIN, 2014) e, estas acima, acabam por transferir o problema de se calcular raízes numericamente para o problema de se calcular integrais numericamente. 


\subsection{Expressões Iterativas para as Raízes das Eqs. (1.5), (1.6) e (1.7)}

Beck et al. (1992) indicaram a utilização do método de Newton-Raphson para a busca de raízes (o qual possui ordem de convergência 2):

$$
\beta_{n}^{k+1}=\beta_{n}^{k}-\frac{f\left(\beta_{n}^{k}\right)}{f^{\prime}\left(\beta_{n}^{k}\right)}
$$

onde, no caso da Eq. (1.5), utilizou-se a função:

$$
f\left(\beta_{n}\right)=\beta_{n} \tan \beta_{n}-c
$$

Obtendo-se:

$$
\beta_{n}^{k+1}=\beta_{n}^{k}-\frac{\beta_{n}^{k} \tan \beta_{n}^{k}-c}{\tan \beta_{n}^{k}+\beta_{n}^{k} \sec ^{2} \beta_{n}^{k}}
$$

Para as raízes da Eq. (1.7), devido a

$$
\beta_{n} \cot \beta_{n}=1-c \rightarrow \beta_{n}=(1-c) \tan \beta_{n}
$$

eles utilizaram a função:

$$
f\left(\beta_{n}\right)=(1-c) \tan \beta_{n}-\beta_{n}
$$

obtendo

$$
\beta_{n}^{k+1}=\beta_{n}^{k}-\frac{(1-c) \tan \beta_{n}^{k}-\beta_{n}^{k}}{\frac{1-c}{\cos ^{2} \beta_{n}^{k}}-1}
$$

onde, ao contrário da Eq. (2.1), não foi utilizado que $\frac{1}{\cos ^{2} \beta_{n}^{k}}=\sec ^{2} \beta_{n}^{k}$.

Também foram apresentadas neste trabalho expressões iterativas para a busca de raízes de outras equações, porém, não se incluiu a Eq. (1.6).

Stevens e Luck (1999) obtiveram funções iterativas de simplicidade e velocidade de convergência muito altas (a ordem de convergência não foi apresentada). Para a da Eq. (1.5), buscase o valor de $\beta_{n}^{k+1}$ tal que:

$$
\beta_{n}^{k+1} \tan \beta_{n}^{k+1}-c=0
$$

que foi reescrita como:

$$
\left(\beta_{n}^{k+1}-\beta_{n}^{k}+\beta_{n}^{k}\right) \tan \left(\beta_{n}^{k+1}-\beta_{n}^{k}+\beta_{n}^{k}\right)-c=0
$$

A expressão de tangente da soma de dois arcos leva a:

$$
\left(\left(\beta_{n}^{k+1}-\beta_{n}^{k}\right)+\beta_{n}^{k}\right) \frac{\tan \left(\beta_{n}^{k+1}-\beta_{n}^{k}\right)+\tan \beta_{n}^{k}}{1-\tan \left(\beta_{n}^{k+1}-\beta_{n}^{k}\right) \tan \beta_{n}^{k}}=c
$$

Para $\left|\beta_{n}^{k+1}-\beta_{n}^{k}\right| \ll 1$ tem-se $\tan \left(\beta_{n}^{k+1}-\beta_{n}^{k}\right) \cong\left(\beta_{n}^{k+1}-\beta_{n}^{k}\right)$, então:

$$
\left(\beta_{n}^{k+1}-\beta_{n}^{k}\right)^{2}+\left((c+1) \tan \beta_{n}^{k}+\beta_{n}^{k}\right)\left(\beta_{n}^{k+1}-\beta_{n}^{k}\right)-\left(c-\beta_{n}^{k} \tan \beta_{n}^{k}\right) \cong 0
$$


Assim:

$$
\beta_{n}^{k+1}=\beta_{n}^{k}+\frac{-B+\sqrt{B^{2}+4 C}}{2}
$$

onde

$$
\begin{gathered}
B=(c+1) \tan \beta_{n}^{k}+\beta_{n}^{k} \\
C=c-\beta_{n}^{k} \tan \beta_{n}^{k}
\end{gathered}
$$

Através da desconsideração do termo $\left(\beta_{n}^{k+1}-\beta_{n}^{k}\right)^{2}$ da equação quadrática, foi obtida uma função iterativa com menor velocidade de convergência, porém mais simples:

$$
\beta_{n}^{k+1}=\beta_{n}^{k}+\frac{C}{B}
$$

Por procedimento análogo, a Eq. (1.6) também leva às Eqs. (2.3) e (2.6) com:

$$
\begin{gathered}
B=\beta_{n}^{k}-c \cot \beta_{n}^{k} \\
C=c-1+\beta_{n}^{k} \tan \beta_{n}^{k}
\end{gathered}
$$

Não existe uma expressão para funções de Bessel da soma, e pudemos obter os coeficientes apresentados neste trabalho, correspondentes às raízes da Eq. (1.7), através de expansão em Taylor de primeira ordem:

$$
\begin{gathered}
B=\frac{2(c-1) \mathrm{J}_{1}\left(\beta_{n}^{k}\right)}{\mathrm{J}_{0}\left(\beta_{n}^{k}\right)-\mathrm{J}_{2}\left(\beta_{n}^{k}\right)}+\beta_{n}^{k} \\
C=\frac{2\left(c \mathrm{~J}_{0}\left(\beta_{n}^{k}\right)-\beta_{n}^{k} \mathrm{~J}_{1}\left(\beta_{n}^{k}\right)\right)}{\mathrm{J}_{0}\left(\beta_{n}^{k}\right)-\mathrm{J}_{2}\left(\beta_{n}^{k}\right)}
\end{gathered}
$$

Estas expressões para os coeficientes $B$ e $C$ são mais complicadas devido a não existir uma definição equivalente à da tangente, para a razão entre as funções de Bessel, conforme já exposto anteriormente.

Apesar de não ser mencionado no trabalho, observamos que a convergência não é atingida para valores altos de $c$ com a utilização da Eq. (2.3), mas apenas com a Eq. (2.6), nem mesmo utilizando estimativas inicias mais exatas do que as que foram utilizadas por eles.

Haji-Sheikh e Beck (2000) indicaram para o cálculo das raízes das Eqs. (1.5) e (1.7), um método de busca de raízes identificado como "Newton-Raphson de alta-ordem":

$$
\beta_{n}^{k+1}=\beta_{n}^{k}-\frac{A_{0}}{A_{1}}\left(1+\frac{\left(A_{2} / A_{1}\right)\left(A_{0} / A_{1}\right)}{1-2\left(A_{2} / A_{1}\right)\left(A_{0} / A_{1}\right)}\right)
$$


onde $A_{m}=f^{(m)}\left(\beta_{n}^{k}\right) / m$ !. Verificou-se que esta função iterativa é a mesma do método de Super-Halley que será discutido posteriormente.

2.2. Propriedades das Raízes das Eqs. (1.5), (1.6) e (1.7)

Para se analisar as expressões aproximadas já publicadas, serão apresentadas algumas propriedades das raízes das Eqs. (1.5), (1.6) e (1.7).

Estas raízes podem ser visualizadas pelas intersecções dos gráficos da Figura 2.1, Figura 2.2 e Figura 2.3, respectivamente, onde pode-se imaginar as linhas contínuas fixas, e as linhas pontilhadas se deslocando para cima ou para baixo conforme $c$ aumenta ou diminui, respectivamente.

Devido a $c \geq 0$, nos gráficos correspondentes às Eqs. (1.5) e (1.6), a linha pontilhada está sempre no primeiro quadrante, enquanto que no gráfico correspondente à Eq. (1.7), ela pode passar por qualquer ponto do quarto quadrante, mas não pode ultrapassar, no primeiro quadrante, a altura correspondente a $c=1$ pois, caso isso ocorra, ela não interceptará a linha contínua, e não haverá raiz real.

Através da variação de $c$, pode-se acompanhar o posicionamento das intersecções que representam as infinitas raízes, e entender os gráficos que estão na Figura 2.4, Figura 2.5 e Figura 2.6, para as raízes das Eqs. (1.5), (1.6) e (1.7), respectivamente, os quais são mais fáceis de se manter em mente enquanto se acompanha o desenvolvimento deste trabalho.

Observe-se o comportamento análogo das raízes das três equações: suas curvas são crescentes e assintóticas, e a diferença que pode ser notada está nos valores das assíntotas horizontais, que podem ser obtidas através dos dois valores extremos de $c$. Substituindo $c=0$ nas Eqs. (1.5), (1.6) e (1.7), obtém-se que os valores de $\beta_{n}(0)$, denotados por $\beta_{n, 0}$, são as $n$-ésimas raízes não negativas de:

$$
\left\{\begin{array}{l}
\operatorname{sen} \beta_{n, 0}=0 \rightarrow \beta_{n, 0}=(\mathrm{n}-1) \pi, \text { para a Eq. (1.5) } \\
\mathrm{J}_{1}\left(\beta_{n, 0}\right)=0 \text {, para a Eq. (1.6) } \\
\beta_{n, 0} \cot \beta_{n, 0}=1 \text {, para a Eq. (1.7) }
\end{array}\right.
$$

Fazendo $c \rightarrow \infty$ nas Eqs. (1.5), (1.6) e (1.7), obtém-se que os valores de $\beta_{n}(\infty)$, denotados por $\beta_{n, \infty}$, são as $n$-ésimas raízes positivas de:

$$
\left\{\begin{array}{l}
\cos \beta_{n, \infty}=0 \rightarrow \beta_{n, \infty}=\left(n-\frac{1}{2}\right) \pi, \text { para a Eq. } \\
\mathrm{J}_{0}\left(\beta_{n, \infty}\right)=0, \text { para a Eq. (1.6) } \\
\operatorname{sen} \beta_{n, \infty}=0 \rightarrow \beta_{n, 0}=n \pi, \text { para a Eq. }(1.7)
\end{array}\right.
$$




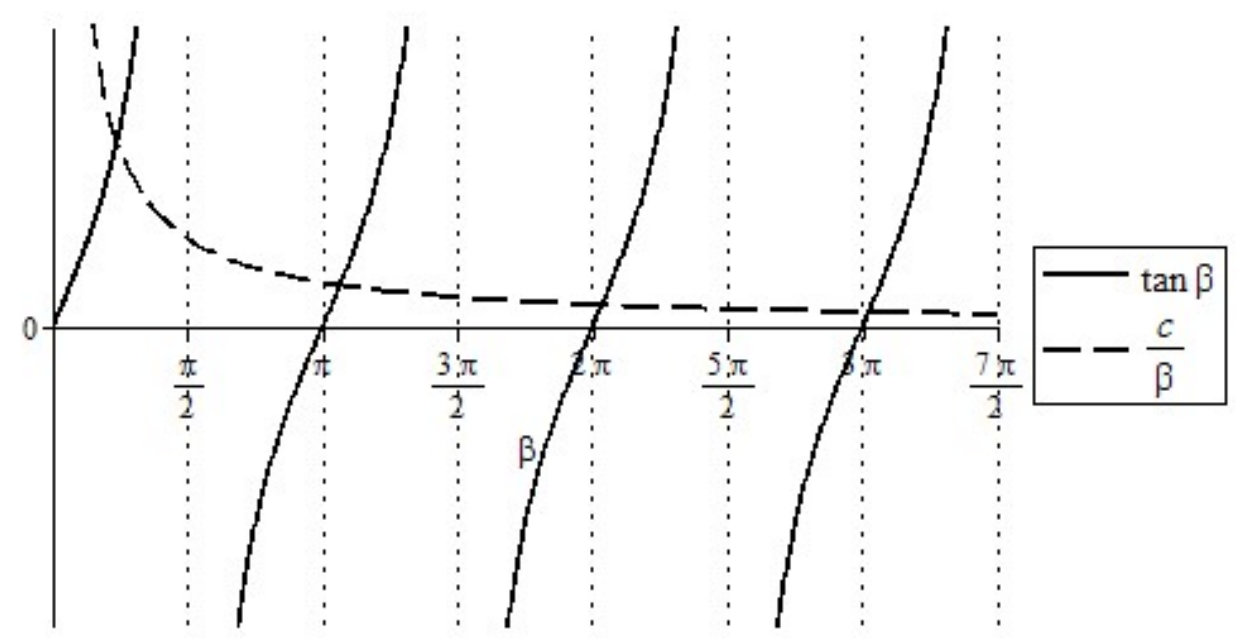

Figura 2.1 Abscissas correspondentes às quatro primeiras raízes da Eq. (1.5)

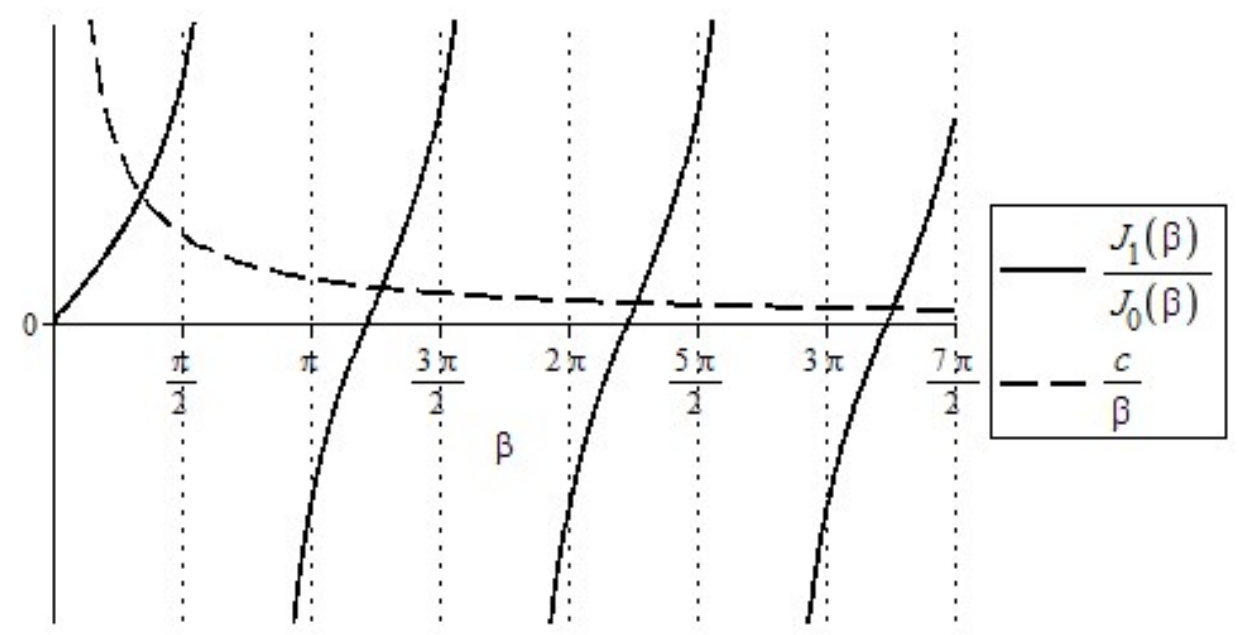

Figura 2.2 Abscissas correspondentes às quatro primeiras raízes da Eq. (1.6)

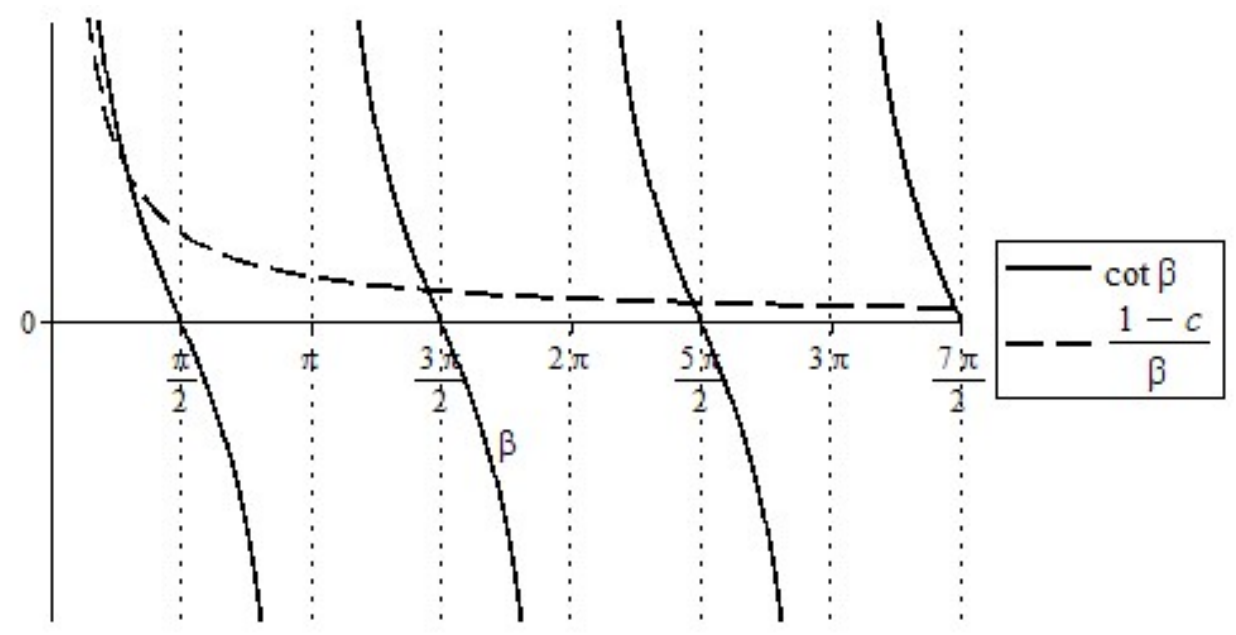

Figura 2.3 Abscissas correspondentes às quatro primeiras raízes da Eq. (1.7) 


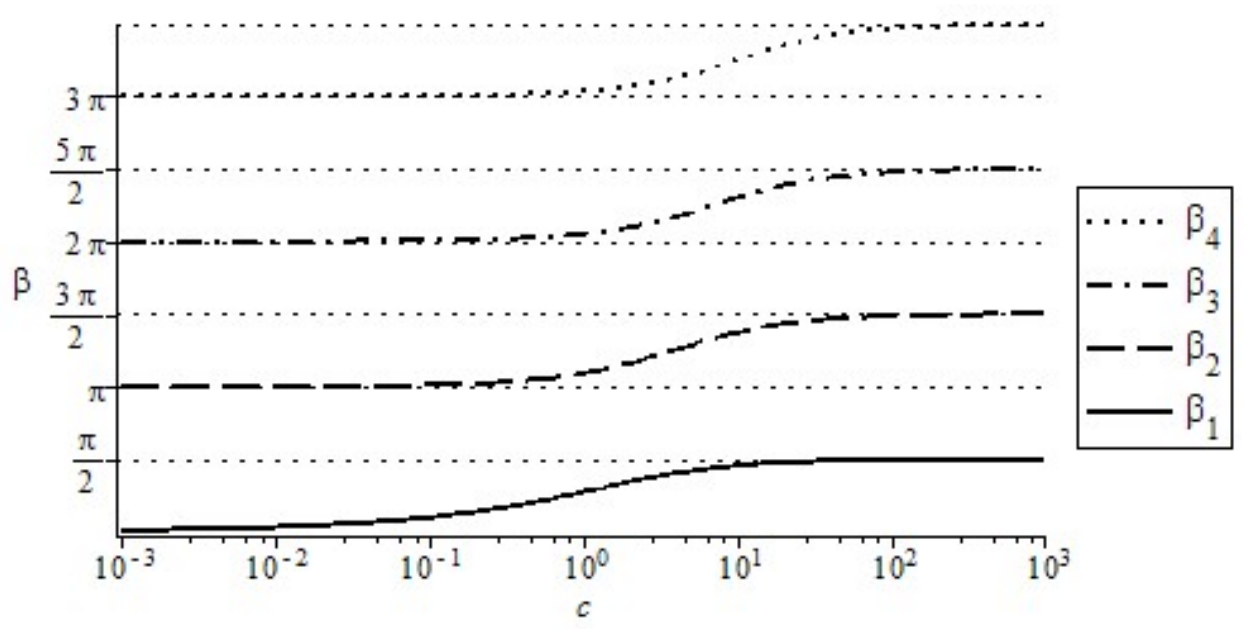

Figura 2.4 Curvas correspondentes às quatro primeiras raízes da Eq. (1.5)

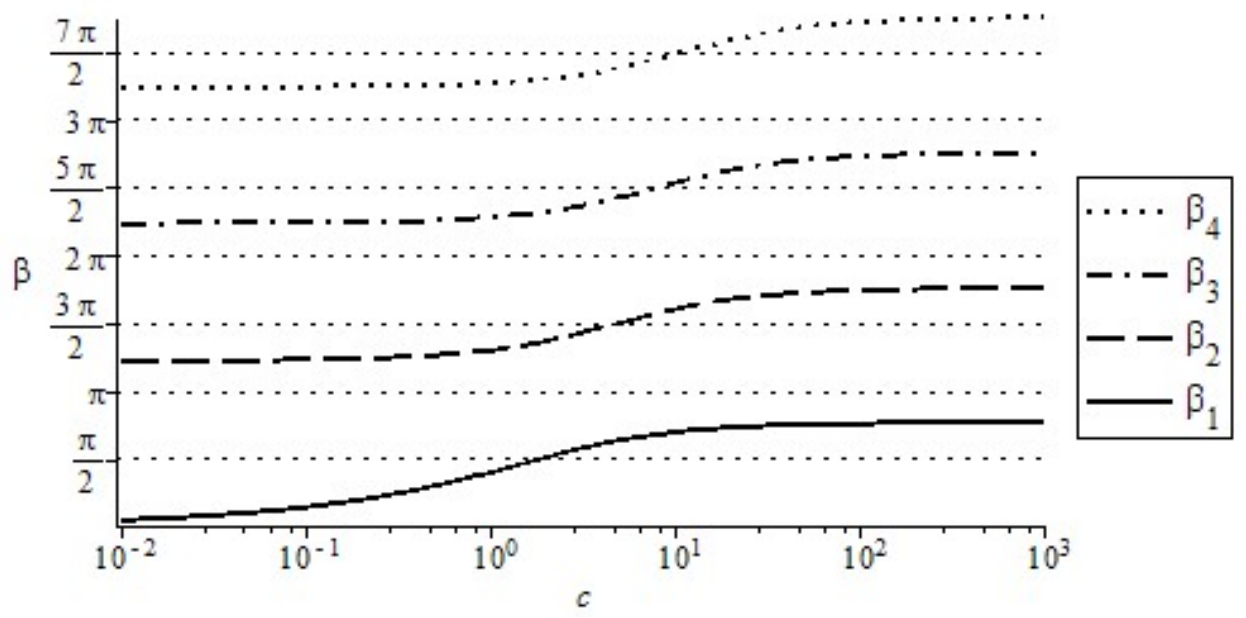

Figura 2.5 Curvas correspondentes às quatro primeiras raízes da Eq. (1.6)

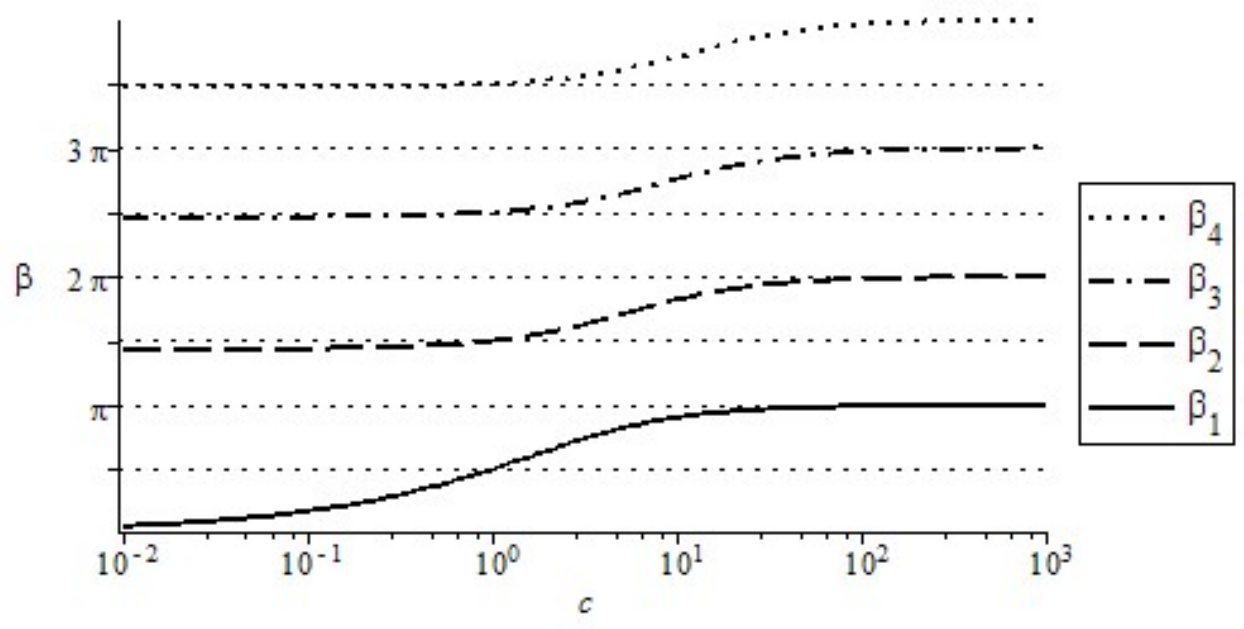

Figura 2.6 Curvas correspondentes às quatro primeiras raízes da Eq. (1.7) 
Esses valores limites estão apresentados na Tabela 2.1, que foi construída de forma a mostrar a analogia entre os valores limites das raízes das três equações.

Ao longo deste trabalho, será utilizado diversas vezes que:

$$
\begin{aligned}
& \beta_{1,0}=0, \text { para as Eq. }(1.5),(1.6) \text { e (1.7) } \\
& \beta_{1, \infty}=\left\{\begin{array}{l}
0,5 \pi \text {, para a Eq. }(1.5) \\
2,4048255, \text { para a Eq. }(1.6) \\
\pi, \text { para a Eq. }(1.7)
\end{array}\right.
\end{aligned}
$$

Tabela 2.1 Valores limites para as raízes das Eqs. (1.5), (1.6) e (1.7)

\begin{tabular}{ccccccc}
\hline & \multicolumn{2}{c}{ Eq. (1.5) } & \multicolumn{2}{c}{ Eq. (1.6) } & \multicolumn{2}{c}{ Eq. (1.7) } \\
\cline { 2 - 5 }$n$ & $\frac{\beta_{n, 0}}{(n-1) \pi}$ & $\frac{\beta_{n, \infty}}{n \pi}$ & $\frac{\beta_{n, 0}}{(n-1) \pi}$ & $\frac{\beta_{n, \infty}}{n \pi}$ & $\frac{\beta_{n, 0}}{(n-1) \pi}$ & $\frac{\beta_{n, \infty}}{n \pi}$ \\
\hline 1 & - & 0,5 & - & 0,7655 & - & 1 \\
2 & 1 & 0,75 & 1,2197 & 0,8785 & 1,4303 & 1 \\
3 & 1 & 0,8333 & 1,1166 & 0,9183 & 1,2295 & 1 \\
4 & 1 & 0,875 & 1,0794 & 0,9383 & 1,1570 & 1 \\
5 & 1 & 0,9 & 1,0603 & 0,9504 & 1,1193 & 1 \\
6 & 1 & 0,9167 & 1,0486 & 0,9587 & 1,0963 & 1 \\
7 & 1 & 0,9286 & 1,0407 & 0,9644 & 1,0807 & 1 \\
8 & 1 & 0,9375 & 1,0350 & 0,9689 & 1,0695 & 1 \\
9 & 1 & 0,9444 & 1,0307 & 0,9721 & 1,0610 & 1 \\
\hline
\end{tabular}

Considere-se as séries de Taylor:

$$
\begin{gathered}
\cos z=\frac{z^{0}}{0 !}-\frac{z^{2}}{2 !}+\frac{z^{4}}{4 !}-\frac{z^{6}}{6 !}+\cdots \\
\operatorname{sen} z=\frac{z^{1}}{1 !}-\frac{z^{3}}{3 !}+\frac{z^{5}}{5 !}-\frac{z^{7}}{7 !}+\cdots \\
\mathrm{J}_{0}(z)=\frac{\left(\frac{1}{4} z^{2}\right)^{0}}{(1 !)^{2}}-\frac{\left(\frac{1}{4} z^{2}\right)^{1}}{(1 !)^{2}}+\frac{\left(\frac{1}{4} z^{2}\right)^{2}}{(2 !)^{2}}-\frac{\left(\frac{1}{4} z^{2}\right)^{3}}{(3 !)^{2}}+\cdots \\
\mathrm{J}_{1}(z)=\frac{1}{2} z\left(\frac{\left(\frac{1}{4} z^{2}\right)^{0}}{0 ! 1 !}-\frac{\left(\frac{1}{4} z^{2}\right)^{1}}{1 ! 2 !}+\frac{\left(\frac{1}{4} z^{2}\right)^{2}}{2 ! 3 !}-\frac{\left(\frac{1}{4} z^{2}\right)^{3}}{3 ! 4 !}+\cdots\right)
\end{gathered}
$$

Utilizando as séries com um termo das funções seno e cosseno na Eq. (1.5), obtém-se:

$$
\frac{\beta_{1} \tan \beta_{1}}{c}=1 \rightarrow \lim _{c \rightarrow 0} \frac{\beta_{1} \tan \beta_{1}}{c}=\lim _{c \rightarrow 0}\left(\frac{\beta_{1}}{c} \frac{\operatorname{sen} \beta_{1}}{\cos \beta_{1}}\right)=\lim _{c \rightarrow 0}\left(\frac{\beta_{1}}{c} \frac{\beta_{1}}{1}\right)=\lim _{c \rightarrow 0}\left(\frac{\beta_{1}^{2}}{c}\right)=1
$$


Então, para a Eq. (1.5):

$$
\beta_{1} \rightarrow \sqrt{c} \text { quando } c \rightarrow 0^{+}
$$

Utilizando as séries de Taylor com um termo das funções de Bessel na Eq. (1.5), obtém-se:

$$
\frac{\beta_{1}}{c} \frac{\mathrm{J}_{1}\left(\beta_{1}\right)}{\mathrm{J}_{0}\left(\beta_{1}\right)}=1 \rightarrow \lim _{c \rightarrow 0}\left(\frac{\beta_{1}}{c} \frac{\mathrm{J}_{1}\left(\beta_{1}\right)}{\mathrm{J}_{0}\left(\beta_{1}\right)}\right)=\lim _{c \rightarrow 0}\left(\frac{\beta_{1}}{c} \frac{\frac{\beta_{1}}{2}}{1}\right)=\lim _{c \rightarrow 0} \frac{\beta_{1}^{2}}{2 c}=c
$$

Então, para a Eq. (1.6):

$$
\beta_{1} \rightarrow \sqrt{2 c} \text { quando } c \rightarrow 0^{+}
$$

Utilizando as séries de Taylor com dois termos das funções seno e cosseno na Eq. (1.7), obtémse:

$$
\begin{aligned}
& \frac{1-\beta_{1} \cot \beta_{1}}{c}=1 \rightarrow \lim _{c \rightarrow 0} \frac{1-\beta_{1} \cot \beta_{1}}{c}=\lim _{c \rightarrow 0} \frac{1-\beta_{1} \frac{\cos \beta_{1}}{\operatorname{sen} \beta_{1}}}{c}= \\
& =\lim _{c \rightarrow 0} \frac{1-\beta_{1} \frac{1-\frac{\beta_{1}^{2}}{2 !}}{\beta_{1}-\frac{\beta_{1}^{3}}{3 !}}}{c}=\lim _{c \rightarrow 0} \frac{\frac{\beta_{1}^{2}}{3}}{c\left(1-\frac{\beta_{1}^{2}}{6}\right)}=1
\end{aligned}
$$

Então, para a Eq. (1.7):

$$
\beta_{1} \rightarrow \sqrt{3 c} \text { quando } c \rightarrow 0^{+}
$$

Portanto

$$
\beta_{1}(c) \rightarrow \sqrt{m c} \text { quando } c \rightarrow 0^{+}
$$

onde $m=1,2$ e 3 para as Eqs. (1.5), (1.6) e (1.7), respectivamente. Esta numeração de $m$ será utilizada ao longo de todo o trabalho. Observe-se a conveniência de utilização de $(1-c)$ em vez de simplesmente $c$ na Eq. (1.7).

Também é possível se obter tendências das demais raízes $\beta_{n}(n=2,3, \ldots)$ quando $c$ se aproxima de zero. Através de derivação implícita das Eq. (1.5) em relação a $c$, obtém-se:

$$
\begin{aligned}
\frac{d}{d c}\left(\beta_{n} \tan \beta_{n}\right) & =\frac{d}{d c} c \rightarrow \frac{d \beta_{n}}{d c} \tan \beta_{n}+\beta_{n} \sec ^{2} \beta_{n} \frac{d \beta_{n}}{d c}=1 \rightarrow \frac{d \beta_{n}}{d c}=\frac{1}{\tan \beta_{n}+\beta_{n} \sec ^{2} \beta_{n}} \\
& =\left.\frac{1}{\tan \beta_{n}+\beta_{n}\left(1+\tan ^{2} \beta_{n}\right)} \rightarrow \frac{d \beta_{n}}{d c}\right|_{c=0}=\frac{1}{\beta_{n, 0}}
\end{aligned}
$$

pois 


$$
c=0 \rightarrow \beta_{n, 0} \tan \beta_{n, 0}=0 \rightarrow \tan \beta_{n, 0}=0
$$

Através de derivação implícita das Eq. (1.6) em relação a $c$, obtém-se:

$$
\begin{aligned}
\frac{d}{d c}\left(\beta_{n} \frac{\mathrm{J}_{1}\left(\beta_{n}\right)}{\mathrm{J}_{0}\left(\beta_{n}\right)}\right) & =\frac{d}{d c} c \rightarrow \frac{\frac{d \beta_{n}}{d c} \mathrm{~J}_{1}\left(\beta_{n}\right) \mathrm{J}_{0}\left(\beta_{n}\right)+\beta_{n} \frac{d \mathrm{~J}_{1}\left(\beta_{n}\right)}{d c} \mathrm{~J}_{0}\left(\beta_{n}\right)-\beta_{n} \mathrm{~J}_{1}\left(\beta_{n}\right) \frac{d \mathrm{~J}_{0}\left(\beta_{n}\right)}{d c}}{\mathrm{~J}_{0}^{2}\left(\beta_{n}\right)}=1 \\
& \rightarrow \frac{\frac{d \beta_{n}}{d c} \mathrm{~J}_{1}\left(\beta_{n}\right) \mathrm{J}_{0}\left(\beta_{n}\right)+\beta_{n}\left(\mathrm{~J}_{0}\left(\beta_{n}\right)-\frac{\mathrm{J}_{1}\left(\beta_{n}\right)}{\beta_{n}}\right) \frac{d \beta_{n}}{d c} \mathrm{~J}_{0}\left(\beta_{n}\right)+\beta_{n} \mathrm{~J}_{1}^{2}\left(\beta_{n}\right) \frac{d \beta_{n}}{d c}}{\mathrm{~J}_{0}^{2}\left(\beta_{n}\right)}=1 \rightarrow \frac{d \beta_{n}}{d c} \\
& =\left.\frac{1}{\beta_{n}\left(1+\frac{\mathrm{J}_{1}^{2}\left(\beta_{n}\right)}{\mathrm{J}_{0}^{2}\left(\beta_{n}\right)}\right)} \rightarrow \frac{d \beta_{n}}{d c}\right|_{c=0}=\frac{1}{\beta_{n, 0}}
\end{aligned}
$$

pois

$$
c=0 \rightarrow \beta_{n} \frac{\mathrm{J}_{1}\left(\beta_{n, 0}\right)}{\mathrm{J}_{0}\left(\beta_{n, 0}\right)}=0 \rightarrow \mathrm{J}_{1}\left(\beta_{n, 0}\right)=0
$$

Através de derivação implícita das Eq. (1.7) em relação a $c$, obtém-se:

$$
\begin{aligned}
\frac{d}{d c}\left(\beta_{n} \cot \beta_{n}\right)= & \frac{d}{d c}(1-c) \rightarrow \frac{d \beta_{n}}{d c} \cot \beta_{n}-\beta_{n} \csc ^{2} \beta_{n} \frac{d \beta_{n}}{d c}=-1 \rightarrow \frac{d \beta_{n}}{d c}=\frac{1}{\beta_{n} \csc ^{2} \beta_{n}-\cot \beta_{n}} \\
& =\left.\frac{1}{\beta_{n}\left(1+\cot ^{2} \beta_{n}\right)-\cot \beta_{n}} \rightarrow \frac{d \beta_{n}}{d c}\right|_{c=0}=\frac{1}{\beta_{n, 0}}
\end{aligned}
$$

pois

$$
c=0 \rightarrow \beta_{n, 0} \cot \beta_{n, 0}=1 \rightarrow \beta_{n, 0} \cot ^{2} \beta_{n, 0}-\cot \beta_{n, 0}=\left(\beta_{n, 0} \cot \beta_{n, 0}\right) \cot \beta_{n, 0}-\cot \beta_{n, 0}=0
$$

Portanto, para todas as Eqs. (1.5), (1.6) e (1.7):

$$
\left.\frac{d \beta_{n}}{d c}\right|_{c=0}=\lim _{c \rightarrow 0} \frac{\beta_{n}(c)-\beta_{n, 0}}{c}=\frac{1}{\beta_{n, 0}} \text { para } n=2,3, \ldots
$$

É possível ocorrer que uma expressão aproximada para as raízes $\beta_{n}$ satisfaça

$$
\left.\frac{d \beta_{n}}{d c}\right|_{c=0}=\frac{1}{\beta_{n, 0}}
$$

sem, no entanto, satisfazer

$$
\lim _{c \rightarrow 0} \frac{\beta_{n}(c)-\beta_{n, 0}}{c}=\frac{1}{\beta_{n, 0}}
$$

apesar de que, por definição de derivada:

$$
\left.\frac{d \beta_{n}}{d c}\right|_{c=0}=\lim _{c \rightarrow 0} \frac{\beta_{n}(c)-\beta_{n, 0}}{c}
$$


Esta diferença, que foi surpreendente à primeira vista, pode ser explicada pelo fato de que a expressão aproximada pode não ter o valor exato $\beta_{n, 0}$ quando $c \rightarrow 0$, quando, então, haverá um $\beta_{n, 0}$ implícito no cálculo da derivada com valor incorreto.

Assim, dispõe-se de algumas ferramentas que serão úteis durante todo este trabalho: os valores que estão na Tabela 2.1 para os quais elas devem se aproximar quando $c$ tende a zero ou infinito, a tendência para a primeira raiz dada pela Eq. (2.12) e a tendência para as demais raízes dada pela Eq. (2.13), sendo que, quando esta última for utilizada para uma expressão aproximada, deve-se calcular o limite com o valor de $\beta_{n, 0}$ correto e não simplesmente a derivada.

\subsection{Expressões Aproximadas para a Primeira Raiz das Eqs. (1.5), (1.6) e (1.7)}

Luikov (1968) apresentou uma expressão fechada aproximada para a primeira raiz $\beta_{1}$ das Eqs. (1.5), (1.6) e (1.7), baseada na aproximada linearidade do gráfico bilogarítmico de $\beta_{1, \infty}^{2} / \beta_{1}^{2}(c)-$ 1 em função de $c$.

Denotando os coeficientes linear e angular da reta por $\log A$ e $-k$, ele obteve:

$$
\log \left(\frac{\beta_{1, \infty}^{2}}{\beta_{1}^{2}(c)}-1\right) \cong \log A-k \log c
$$

A potência $\beta_{1}^{2}(c)$ que aparece nessa expressão está no texto, porém não está presente no gráfico da Figura 6.31 desta referência, provavelmente devido a erro de tipografia. Yovanovich (1996) reproduziu este erro, e todas demais referências subsequentes que mencionam este trabalho também o reproduziram. Enfatiza-se esse fato apenas para que não surjam dúvidas se houver alguma comparação do presente trabalho com os demais.

Simplificando essa equação e usando métodos de ajuste de curvas, ele obteve:

$$
\beta_{1} \cong \frac{\beta_{1, \infty}}{\sqrt{1+\frac{A}{c^{k}}}}
$$

onde $A=2,24,2,45$ e 2,70, e $k=1,02,1,04$ e 1,07, para a primeira raiz das Eqs. (1.5), (1.6) e (1.7), respectivamente.

As curvas com os valores de $\beta_{1}$ calculados por esta expressão aproximada, como função da variável independente $c$, tem pequenas diferenças em relação às curvas com os valores exatos (obtidos através de métodos iterativos) de $\beta_{1}$ como pode ser visto na Figura 2.7 . 


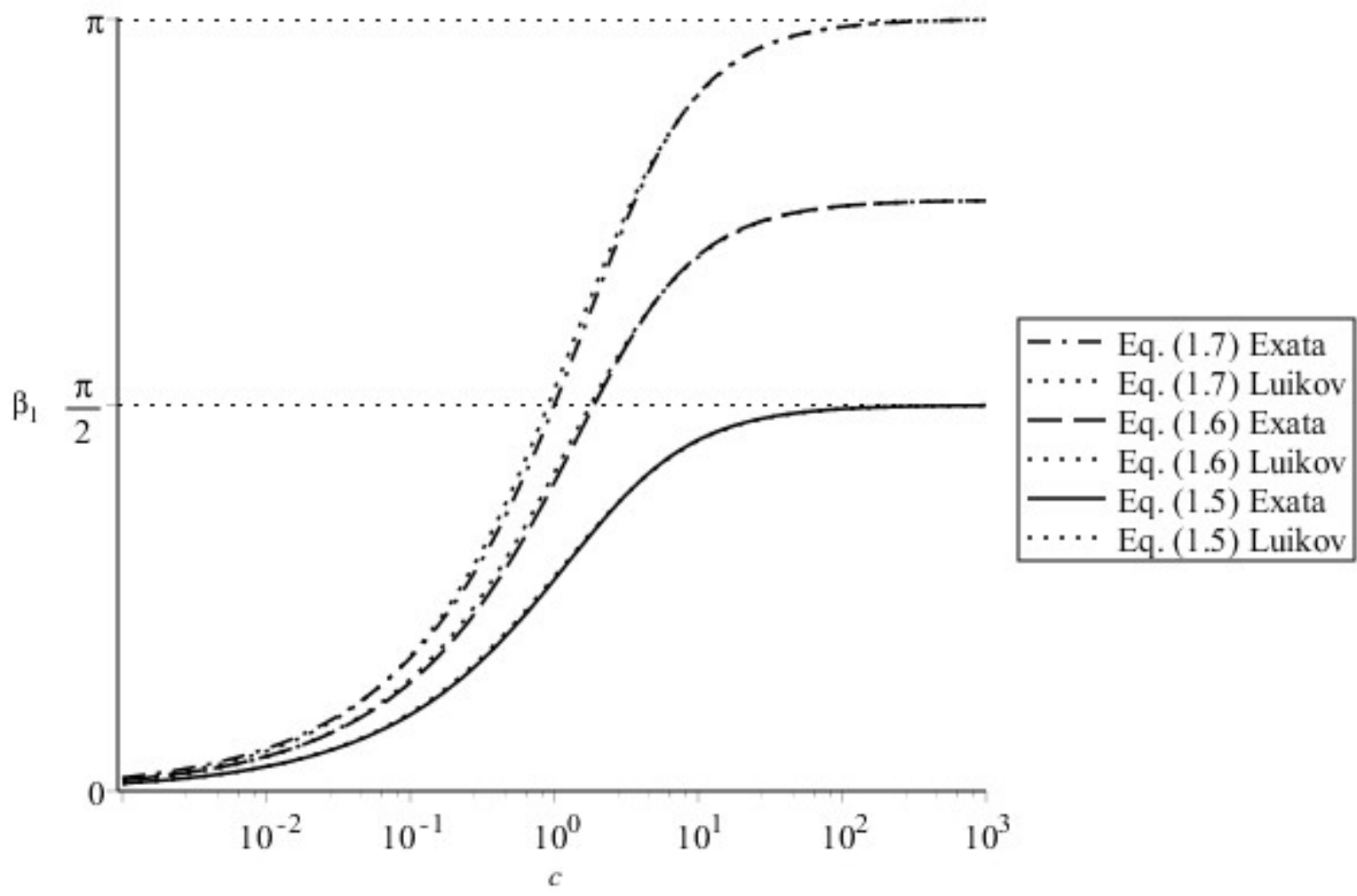

Figura 2.7 Valores aproximados obtidos pela Eq. (2.14) de Luikov (1968) e valores exatos da primeira raiz das Eqs. (1.5), (1.6) $e(1.7)$

Pode-se notar que esta expressão tende aos valores corretos quando $c$ tende a zero ou infinito:

$$
\begin{gathered}
\lim _{c \rightarrow 0} \frac{\beta_{1, \infty}}{\sqrt{1+A / c^{k}}}=0=\beta_{1,0} \\
\lim _{c \rightarrow \infty} \frac{\beta_{1, \infty}}{\sqrt{1+A / c^{k}}}=\beta_{1, \infty}
\end{gathered}
$$

então o erro absoluto das raízes dadas por esta expressão tende a zero quando $c$ tende a zero ou infinito, e as curvas do gráfico da Figura 2.8 tem o eixo horizontal como assíntota para ambos lados das três curvas.

Porém, quando se trata de erro relativo:

$$
\frac{\beta_{1}^{\text {aproximado }}-\beta_{1}^{\text {exato }}}{\beta_{1}^{\text {exato }}}
$$

os resultados não são necessariamente os mesmos, como pode-se observar na Figura 2.9. 0 fato de que essa expressão tenda para o valor correto quando $c$ tende a infinito, implica que o erro relativo 


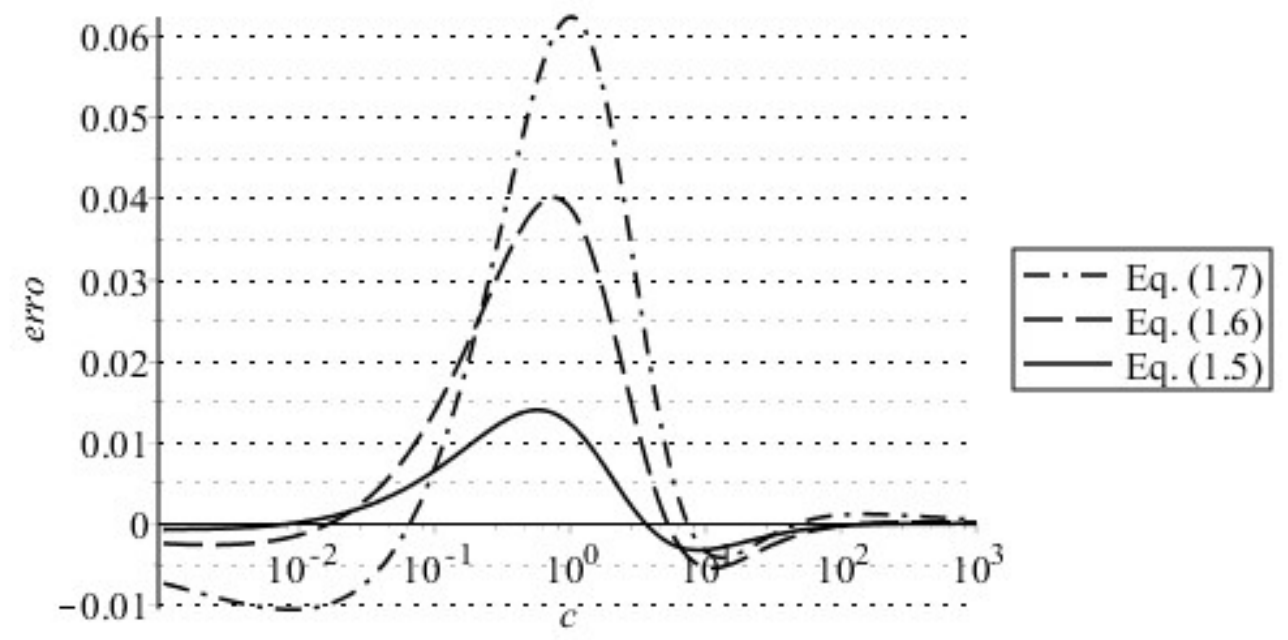

Figura 2.8 Erro dos valores obtidos pela Eq. (2.14) de Luikov (1968) para a primeira raiz das Eqs. (1.5), (1.6) e (1.7)

também tenda a zero, pois, quando o limite de uma função que tende a zero é dividido por uma função que não tende a zero, o quociente resultante tenderá a zero:

$$
\lim _{c \rightarrow \infty} \frac{\beta_{1, \infty}}{\sqrt{1+A / c^{k}}}=\beta_{1, \infty} \rightarrow \lim _{c \rightarrow \infty} \frac{\frac{\beta_{1, \infty}}{\sqrt{1+\frac{A}{c^{k}}}}-\beta_{1, \infty}}{\beta_{1, \infty}}=0
$$

Então o eixo horizontal continua sendo assíntota para o lado direito das curvas, quando o erro calculado é o relativo.

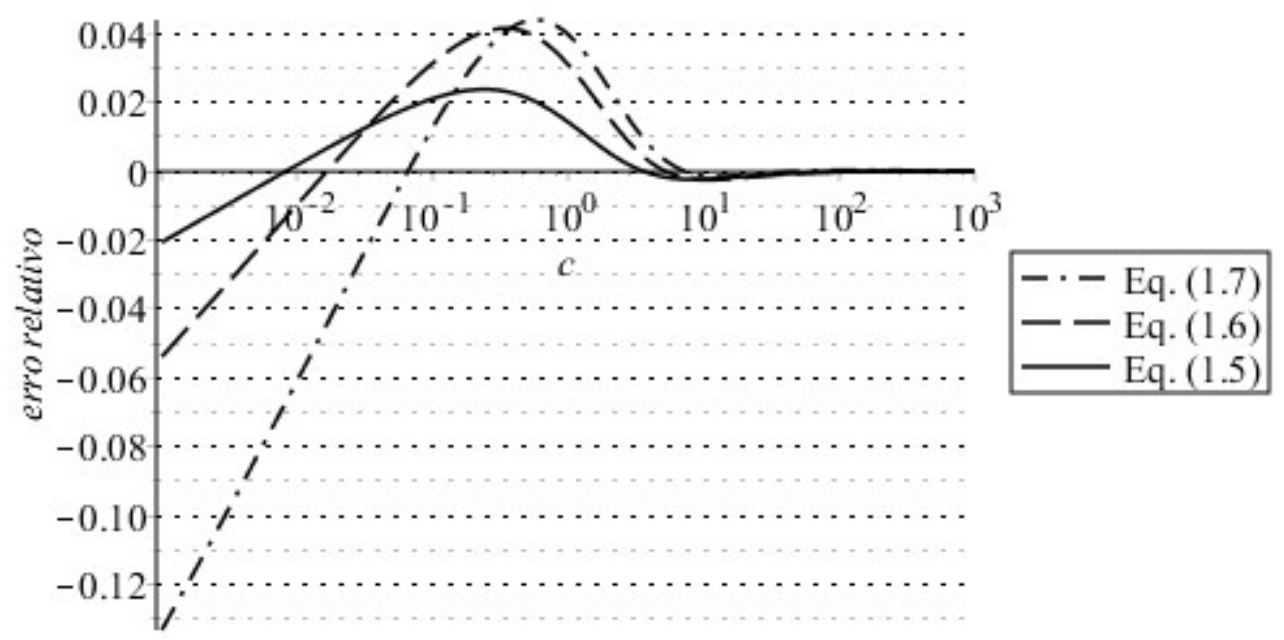

Figura 2.9 Erro relativo dos valores obtidos pela Eq. (2.14) de Luikov (1968) para a primeira raiz das Eqs. (1.5), (1.6) e (1.7) 
Porém, essas curvas não têm mais o eixo horizontal como assíntota para o lado esquerdo, e o raciocínio anterior não é aqui válido, devido ao valor correto ser nulo quando $c$ tende a zero, o que levaria a uma divisão de zero por zero e, consequentemente, uma indeterminação.

Pode-se ver que essa expressão não satisfaz a tendência dada pela Eq. (2.12):

$$
\lim _{c \rightarrow 0^{+}} \frac{\frac{\beta_{1, \infty}}{\sqrt{1+A / c^{k}}}}{\sqrt{m c}}=\lim _{c \rightarrow 0^{+}} \frac{\beta_{1, \infty}}{\sqrt{m c+A m / c^{k-1}}}=0 \neq 1
$$

e, como consequência deste limite dar zero em vez de um, o erro relativo tende a $-100 \%$ quando $c$ tende a zero:

$$
\lim _{c \rightarrow 0^{+}} \frac{\frac{\beta_{1, \infty}}{\sqrt{1+A / c^{k}}}-\sqrt{m c}}{\beta_{1,0}}=\lim _{c \rightarrow 0^{+}} \frac{\frac{\beta_{1, \infty}}{\sqrt{1+A / c^{k}}}-\sqrt{m c}}{\sqrt{m c}} \frac{\sqrt{m c}}{\beta_{1,0}}=-1
$$

o que significa que a assíntota para o lado esquerdo de todas as curvas da Figura 2.9 é a reta horizontal que passa pelo ponto -1 do eixo vertical. Um gráfico assim foi obtido, mas não será inserido aqui devido a não incluir informação relevante além dessa já descrita.

Devido ao erro relativo ser mais relevante, gráficos comparativos como o da Figura 2.7, e gráficos de erros absolutos como o da Figura 2.8 não serão novamente apresentados.

Os módulos dos erros relativos são mostrados no capítulo de comparação de expressões e, em particular, os erros que são apresentados na Figura 2.9 tem seus módulos mostrados nos gráficos comparativos da Figura 8.1, Figura 8.2 e Figura 8.3, para as Eqs. (1.5), (1.6) e (1.7), respectivamente. Então, estes gráficos não serão repetidos neste capítulo, mas apenas no capítulo de comparação, para as próximas expressões.

Por fim, a utilização de módulos facilitará a comparação entre os erros das expressões, já que erros relativos positivos e negativos têm mesma importância. Apenas deve-se atentar para o fato de que um erro relativo negativo tem seus valores limitados a $-100 \%$, enquanto que um erro relativo positivo tem valores ilimitados.

Beck et al. (1992) apresentaram várias expressões aproximadas em forma fechada para todas raízes das Eqs. (1.5) e (1.7), sem indicarem, no entanto, como elas foram obtidas. Para a primeira raiz da Eq. (1.5), eles apresentaram:

$$
\beta_{1} \cong\left\{\begin{array}{l}
\sqrt{\frac{3 c}{3+c}\left(1-\frac{1}{45}\left(\frac{3 c}{3+c}\right)^{2}\right)}, 0 \leq c \leq 2 \\
\frac{\pi c}{2(c+1)}\left(1+\frac{\pi^{2}}{12(c+1)^{3}+\pi^{2}(2 c-1)}\right), c>2
\end{array}\right.
$$


com erro relativo máximo de $0,1 \%$, segundo o trabalho

Para a primeira raiz da Eq. (1.7), eles apresentaram:

$$
\beta_{1} \cong\left\{\begin{array}{l}
\sqrt{3\left(c-c^{2} / 5\right)}, 0 \leq c<0,4 \\
\frac{1}{2} \pi\left(1+\frac{3}{2(c+2)}\left(\sqrt{1+\frac{16(c-1)(c+2)}{3 \pi^{2}}}-1\right)\right), 0,4 \leq c<6 \\
\pi-\sqrt[3]{a+\frac{3 \pi}{2(c-1)}}+\sqrt[3]{a-\frac{3 \pi}{2(c-1)}}, c>6
\end{array}\right.
$$

onde

$$
a=\sqrt{\left(\frac{3 \pi}{2(c-1)}\right)^{2}+\left(\frac{c}{c-1}\right)^{3}}
$$

Ao contrário da Eq. (2.15), o erro da Eq. (2.16) não é indicado.

A expressão da Eq. (2.15) para a primeira raiz da Eq. (1.5) com menores valores de $c$ satisfaz a tendência dada pela Eq. (2.12):

$$
\lim _{c \rightarrow 0^{+}}\left(\frac{\sqrt{\frac{3 c}{3+c}\left(1-\frac{1}{45}\left(\frac{3 c}{3+c}\right)^{2}\right)}}{\sqrt{c}}\right)=1
$$

então ela tem erro relativo tendendo a zero quando $c$ tende a zero, como já exposto para a expressão anterior e, consequentemente, ela tem erro absoluto também tendendo a zero quando $c$ tende a zero, o que equivale a:

$$
\lim _{c \rightarrow 0} \sqrt{\frac{3 c}{3+c}\left(1-\frac{1}{45}\left(\frac{3 c}{3+c}\right)^{2}\right)}=0=\beta_{1,0}
$$

Essa consequência é devida ao fato de que a existência do limite da divisão de uma função por outra que tende a zero implicar no valor da primeira função também tender a zero:

$$
\lim _{c \rightarrow 0} \sqrt{\frac{3 c}{3+c}\left(1-\frac{1}{45}\left(\frac{3 c}{3+c}\right)^{2}\right)}=\lim _{c \rightarrow 0^{+}} \frac{\sqrt{\frac{3 c}{3+c}\left(1-\frac{1}{45}\left(\frac{3 c}{3+c}\right)^{2}\right)}}{\sqrt{c}} \cdot \lim _{c \rightarrow 0^{+}} \sqrt{c}=0
$$


Estas conclusões sobre erros absolutos e relativos serão utilizadas nas próximas análises, então elas serão destacadas para facilitar a leitura deste texto. A primeira conclusão é que se uma expressão tende ao valor correto quando $c$ tende a infinito, então ela obrigatoriamente tem erro relativo também tendendo a zero quando $c$ tende a infinito. A segunda conclusão é que se uma expressão satisfaz à tendência dada pela Eq. (2.12), então ela tem erro relativo tendendo a zero quando $c$ tende a zero. Por fim, quando o erro relativo tende a zero, o erro absoluto também tende a zero, então, quando uma expressão tiver erro relativo tendendo a zero, não será repetido que seu erro absoluto também tende a zero.

A expressão da Eq. (2.15) para a primeira raiz da Eq. (1.5) com maiores valores de $c$ satisfaz o valor assintótico quando $c$ tende a infinito:

$$
\lim _{c \rightarrow \infty}\left(\frac{\pi c}{2(c+1)}\left(1+\frac{\pi^{2}}{12(c+1)^{3}+\pi^{2}(2 c-1)}\right)\right)=\frac{\pi}{2}=\beta_{1, \infty}
$$

então, ela tem erro relativo tendendo a zero quando $c$ tende a infinito, o que pode ser visto através da Figura 8.13.

A expressão da Eq. (2.16) para a primeira raiz da Eq. (1.7) satisfaz a tendência dada pela Eq. (2.12):

$$
\lim _{c \rightarrow 0^{+}} \frac{\sqrt{3\left(c-c^{2} / 5\right)}}{\sqrt{3 c}}=1
$$

e tem o valor correto quando $c$ tende a infinito:

$$
\lim _{c \rightarrow \infty}\left(\pi-\sqrt[3]{a+\frac{3 \pi}{2(c-1)}}+\sqrt[3]{a-\frac{3 \pi}{2(c-1)}}\right)=\pi=\beta_{n, \infty}
$$

então tem erro relativo tendendo a zero quando $c$ tende a zero ou infinito, o pode ser visto através da Figura 8.12.

Churchill e Usagi (1972) apresentaram uma expressão "consideravelmente bem-sucedida" para ajuste de valores experimentais ou computacionais, de uma variável dependente $y$ em função de uma variável dependente $x$ :

$$
y(x) \cong\left(\left(y_{0}(x)\right)^{r}+\left(y_{\infty}(x)\right)^{r}\right)^{\frac{1}{r}}
$$


onde $y_{0}(x)$ e $y_{\infty}(x)$ são expressões assintóticas para $y$, conforme $x$ tende a zero ou infinito, respectivamente, e $r$ é o parâmetro de ajuste. Yovanovich (1996) utilizou essa expressão para as presentes equações, definindo $k=-r$, e utilizando a forma:

$$
y(x) \cong \frac{1}{\left(\frac{1}{\left(y_{0}(x)\right)^{k}}+\frac{1}{\left(y_{\infty}(x)\right)^{k}}\right)^{\frac{1}{k}}}
$$

Ele substituiu a expressão assintótica da Eq. (2.12) e os valores exatos quando $c$ tende a infinito nesta relação, obtendo diretamente a seguinte expressão para a primeira raiz:

$$
\beta_{1} \cong \frac{\beta_{1, \infty}}{\left(1+\left(\frac{\beta_{1, \infty}}{\sqrt{m c}}\right)^{k}\right)^{\frac{1}{k}}}
$$

a qual foi ajustada para valores de $c$ de 0,5 a 5,0, levando a $k=2,139,2,238$ e 2,314 para as Eqs. (1.5), (1.6) a (1.7), respectivamente.

Pode-se notar que essa expressão satisfaz a tendência dada pela Eq. (2.12), e tem o valor correto quando $c$ tende a infinito e, então, tem erro relativo tendendo a zero quando $c$ tende a zero ou infinito. Assim, o erro máximo ocorre num ponto intermediário entre esses extremos, o que pode ser vistos na Figura 8.10, Figura 8.11, Figura 8.12 para as Eqs. (1.5), (1.6) a (1.7), respectivamente. Ela tem a mesma forma da expressão de Luikov (1968), porém ela tem a raiz quadrada substituída por $1 / k$, o que fez seus resultados serem muito mais exatos.

Esta expressão, segundo o autor, "proporciona valores de $\beta_{1}$ que diferem menos de $0,4 \%$ dos valores exatos de $\beta_{1}$. Esta exatidão é aceitável para a maioria das aplicações." Pelos gráficos construídos observamos que, na verdade, esta diferença é de até $0,7 \%$. Pode-se confirmar isso, substituindo $c=4$ na expressão da Eq. (2.19) para o caso da raiz da Eq. (1.7):

$$
\beta_{1} \cong \frac{\pi}{\left(1+\left(\frac{\pi}{\sqrt{3 \cdot 0,4}}\right)^{2,314}\right)^{\frac{1}{2,314}}}=2,43827
$$

e ver pela Tabela 4.3 o valor correto é 2,4556 , o que confirma o erro mostrado pelo gráfico.

Stevens e Luck (1999) apresentaram expressões aproximadas para todas raízes das Eqs. (1.5), (1.6) e (1.7), bastante simples mas com pouca exatidão, para que elas sirvam apenas como pontos iniciais de métodos iterativos. A primeira raiz da Eq. (1.5) é aproximada por: 


$$
\beta_{1} \cong \frac{\pi}{4}+\frac{\pi}{4} \frac{c-\frac{\pi}{4}}{c+\frac{\pi}{4}}
$$

A primeira raiz da Eq. (1.6) é aproximada por:

$$
\beta_{1} \cong \frac{3 \pi}{8}+\frac{3 \pi}{8} \frac{c-\frac{\pi}{2}}{c+\frac{\pi}{2}}
$$

E a primeira raiz da Eq. (1.7) é aproximada por:

$$
\beta_{1} \cong \frac{\pi}{2}+\frac{\pi}{2} \frac{c^{0,676}-\frac{\sqrt{\pi}}{2}}{c^{0,676}+\frac{\sqrt{\pi}}{2}}
$$

A origem destas equações não foi mostrada, e um erro tipográfico na Eq. (2.21) foi constatado e corrigido a partir de comparação de simulações destas expressões com os resultados publicados.

Repetindo-se os cálculos de limites que foram feitos anteriormente para se verificar as equações desenvolvidas nos outros trabalhos para estas equações, obtém-se que estas expressões não têm a tendência correta da Eq. (2.12), e tem erro relativo tendendo a $-100 \%$ quando $c$ tende a zero. Note que este valor de $-100 \%$ é um limite que poderia ser atingido apenas se o valor aproximado fosse nulo ao mesmo tempo em que o valor exato fosse não nulo.

Também obtém-se que o erro relativo destas expressões tende a zero quando $c$ tende a zero ou infinito, com exceção da primeira raiz da Eq. (1.6) dada pela Eq. (2.21), que tem erro que tende aproximadamente a zero quando $c$ tende a infinito. Esta aproximação da Eq. (1.6) é satisfatória e utiliza que $\beta_{1, \infty}=2,4048255=0,7655 \pi \cong 0,75 \pi$. É claro que nenhuma expressão poderá levar a este valor exato, já que ele é a solução de uma equação que não tem solução em forma fechada, exceto, é claro, as expressões que já contiverem o valor

Através da Figura 8.1, Figura 8.2 e Figura 8.3, para as Eqs. (1.5), (1.6) e (1.7), respectivamente, pode-se ver o erro relativo das raízes calculadas por estas expressões. Note-se que o ponto de intersecção da curva correspondente à Eq. (2.20) representa o ponto fixo no qual $\beta_{1}=\pi / 4$ quando $c=\pi / 4$.

Haji-Sheikh e Beck (2000) se propuseram a melhorar as expressões de Stevens e Luck (1999). Para a Eq. (2.20) eles procuraram incluir a tendência dada pela Eq. (2.12), obtendo que a primeira raiz da Eq. (1.5) pode ser aproximada por: 


$$
\beta_{1} \cong \frac{\pi}{4}+\frac{\pi}{4} \frac{c-\pi / 4}{c+\pi / 4}\left(1-1,04\left(\sqrt{\frac{c}{c+0,76}}-\frac{c}{c+0,76}\right)\right)
$$

Novamente com a Eq. (2.20), através de translações horizontal e vertical, eles obtiveram que a primeira raiz da Eq. (1.7) pode ser aproximada por:

$$
\beta_{1} \cong \frac{3 \pi}{4}+\frac{\pi}{4} \frac{c-1-3 \pi / 4}{c-1+3 \pi / 4}
$$

A primeira raiz da Eq. (1.5) calculada pela Eq. (2.23) tem erro relativo tendendo a $-6,4 \%$ quando $c$ tende a zero, conforme pode ser visto por:

$$
\lim _{c \rightarrow 0^{+}} \frac{\frac{\pi}{4}+\frac{\pi}{4} \frac{c-\frac{\pi}{4}}{c+\frac{\pi}{4}}\left(1-1,04\left(\sqrt{\frac{c}{c+0,76}}-\frac{c}{c+0,76}\right)\right)}{\sqrt{c}}=0,937
$$

Então, o objetivo de se incluir a tendência dada pela Eq. (2.12) não foi plenamente atingido.

Um erro absoluto que tende a zero quando $c$ tende a zero é consequência da existência desse limite. Ela tem erro relativo tendendo a zero quando $c$ tende a infinito, conforme pode ser obtido por cálculo análogo aos já mostrados. Na Figura 8.1 o erro dessa expressão é mostrado, e a curva correspondente tem como assíntota à esquerda a reta horizontal que passa pelo ponto de ordenada 0,063 .

Também se obtém que a primeira raiz da Eq. (1.7) calculada pela Eq. (2.24) tem erro absoluto tendendo a 0,41 (o que implica em erro relativo tendendo a infinito) quando $c$ tende a zero, e tem erro relativo tendendo a zero quando $c$ tende a infinito (Figura 8.3).

Ostrogorsky e Mikic (2008) desenvolvem expressões aproximadas para a primeira raiz das três equações. Inicialmente eles substituíram $\tan \beta_{1}$ da Eq. (1.5) por sua série de Maclaurin com dois termos, obtendo:

$$
\beta_{1}^{2}\left(1+\beta_{1}^{2} / 3\right) \cong c
$$

Se o $\beta_{1}$ desta equação é isolado, a expressão resultante não tem boa exatidão, mas se o segundo $\beta_{1}$ desta equação for substituído por seu valor assintótico dado pela Eq. (2.12), obtém-se:

$$
\beta_{1} \cong \sqrt{\frac{c}{1+c / 3}}
$$

Essa expressão tem ela mesma a tendência dada pela Eq. (2.12), mas por não ter valor correto quando $c$ tende a infinito, foi modificada para: 


$$
\beta_{1} \cong \sqrt{\frac{c}{1+\frac{c}{\beta_{1, \infty}^{2}}}}
$$

Deduções análogas foram feitas a partir das Eqs. (1.6) e (1.7), e todos resultados podem ser colocados numa única expressão:

$$
\beta_{1} \cong \sqrt{\frac{m c}{1+\frac{m c}{\beta_{1, \infty}^{2}}}}
$$

onde $m=1$, 2 e 3 para as Eqs. (1.5), (1.6) e (1.7), respectivamente.

Esta expressão tem as tendências corretas e o erro relativo tendendo a zero nos dois extremos de $c$. Sua exatidão não é a menor (Figura 8.4, Figura 8.5 e Figura 8.6, para a primeira raiz das Eqs. (1.5), (1.6) e (1.7), respectivamente), e seu mérito está em sua simplicidade; observe-se que ela nem mesmo contém valores de ajuste.

Ostrogorsky e Mikic (2009) desenvolvem mais expressões aproximadas para a primeira raiz das três equações. Para o caso em que $c \geq 2$, eles utilizam uma das formas mais comuns de ajuste, a expressão racional $1 \mathrm{x} 1$, sobre a qual aplicaram os valores exatos nos limites extremos. Para $c<2$ eles mantêm a expressão da Eq. (2.25) sem a necessidade da correção para $\beta_{1, \infty}$ e, então:

$$
\beta_{1} \cong\left\{\begin{array}{l}
\sqrt{\frac{m c}{1+\frac{c}{m+2}}}, 0 \leq c<2 \\
\beta_{1, \infty} \frac{c}{c+a}, c \geq 2
\end{array}\right.
$$

onde $a=0,95,1,0$ e 1,1, para as Eqs. (1.5), (1.6) e (1.7), respectivamente.

Esta expressão tem as tendências corretas e o erro relativo tendendo a zero nos dois extremos, o que pode ser visto na Figura 8.7, Figura 8.8 e Figura 8.9, para a primeira raiz das Eqs. (1.5), (1.6) e (1.7), respectivamente.

Ostrogorsky (2010) modificou os parâmetros da expressão da Eq. (2.25) para obter melhor ajuste para $c>0,1$ e utilizar a tendência dada pela Eq. (2.12) para os menores valores de $c$, obtendo:

$$
\beta_{1} \cong\left\{\begin{array}{l}
\sqrt{m c}, 0 \leq c \leq 0.1 \\
\frac{\beta_{1, \infty}}{\sqrt{1+\frac{a}{c^{k}}}}, c>0.1
\end{array}\right.
$$

onde $a=2,3,2,62$ e 2,92, e $k=1,035,1,05$ e 1,08 para as Eqs. (1.5), (1.6) e (1.7), respectivamente. 
Esta expressão tem as tendências corretas e o erro relativo tendendo a zero nos dois extremos, e está identificada como "Ostrogorsky (2010a)" na Figura 8.7, Figura 8.8 e Figura 8.9, para a primeira raiz das Eqs. (1.5), (1.6) e (1.7), respectivamente.

Esse trabalho também reapresentou a expressão (2.19) com novos parâmetros que levam a um melhor ajuste para $c>0,1$ :

$$
\beta_{1} \cong \frac{\beta_{1, \infty}}{\left(1+\frac{a}{c^{k}}\right)^{d}}
$$

onde $a=2,62,3,28$ e 4,1, $k=1,07,1,125$ e 1,18, e $d=0,468,0,446$ e 0,4238 para as Eqs. (1.5), (1.6) e (1.7), respectivamente.

Através de cálculos análogos aos já apresentados, obtém-se que esta expressão tem as tendências corretas apenas no extremo correspondente a $c$ tendendo a infinito. Quando $c$ tende a zero, o erro relativo tende a $-100 \%$, mas de maneira lenta como pode ser visto na Figura 8.10, Figura 8.11, Figura 8.12, para a primeira raiz das Eqs. (1.5), (1.6) e (1.7), respectivamente, onde ela está identificada como "Ostrogorsky (2010b)".

\subsection{Expressões Aproximadas para as Demais Raízes das Eqs. (1.5), (1.6) e (1.7)}

Beck et al. (1992) apresentaram para as demais raízes da equação (1.5):

$$
\beta_{n} \cong\left\{\begin{array}{l}
\frac{(n-1) \pi}{2(c+3)}\left(2 c+3+3 \sqrt{1+\frac{4 c(c+3)}{3(n-1)^{2} \pi^{2}}}\right), c \text { menor } \\
\frac{(2 n-1) \pi c}{2(c+1)}\left(1+\frac{(2 n-1)^{2} \pi^{2}}{12(c+1)^{3}+(2 n-1)^{2} \pi^{2}(2 c-1)}\right), c \text { maior }
\end{array}\right.
$$

onde a divisão do intervalo de $c$ deve ocorrer em $c=5,8,11$ e 13 para $n=2,3$, 4 e 5, respectivamente. Eles indicam que o erro relativo máximo desta expressão é de $0,23 \%$ para $\beta_{2}$, e $0,1 \%$ para $\beta_{3}, \beta_{4}$ e $\beta_{5}$, e não mostram pontos de divisão para as demais raízes.

Para todas raízes da Eq. (1.7), eles apresentaram:

$$
\beta_{n} \cong\left\{\begin{array}{l}
\left(n-\frac{1}{2}\right) \pi\left(1+\frac{3}{2(c+2)}\left(\sqrt{1+\frac{16(c-1)(c+2)}{3(2 n-1)^{2} \pi^{2}}}-1\right)\right), c<6 \\
n \pi-\sqrt[3]{a+\frac{3 n \pi}{2(c-1)}}+\sqrt[3]{a-\frac{3 n \pi}{2(c-1)}}, c \geq 6
\end{array}\right.
$$


onde

$$
a=\sqrt{\left(\frac{3 n \pi}{2(c-1)}\right)^{2}+\left(\frac{c}{c-1}\right)^{3}}
$$

Ao contrário do erro da Eq. (2.29), o erro da Eq. (2.30) não é indicado.

A expressão da Eq. (2.29) para as demais raízes da Eq. (1.5) satisfaz a Eq. (2.13):

$$
\lim _{c \rightarrow 0} \frac{\frac{(n-1) \pi}{2(c+3)}\left(2 c+3+3 \sqrt{1+\frac{4 c(c+3)}{3(n-1)^{2} \pi^{2}}}\right)-(n-1) \pi}{c}=\frac{1}{\beta_{n, 0}}
$$

e a existência deste limite implica em erro relativo tendendo a zero:

$$
\lim _{c \rightarrow 0} \frac{\beta_{n}(c)-\beta_{n, 0}}{\beta_{n, 0}}=\lim _{c \rightarrow 0} \frac{\beta_{n}(c)-\beta_{n, 0}}{c} \lim _{c \rightarrow 0} \frac{c}{\beta_{n, 0}}=\left.\frac{d \beta_{n}}{d c}\right|_{c=0} \cdot 0=0
$$

e também em erro absoluto tendendo a zero, como pode ser visto por:

$$
\lim _{c \rightarrow 0}\left(\frac{(n-1) \pi}{2(c+3)}\left(2 c+3+3 \sqrt{1+\frac{4 c(c+3)}{3(n-1)^{2} \pi^{2}}}\right)\right)=(n-1) \pi=\beta_{n, 0}
$$

Ela também tem o valor correto quando $c$ tende a infinito:

$$
\lim _{c \rightarrow \infty}\left(\frac{(2 n-1) \pi c}{2(c+1)}\left(1+\frac{(2 n-1)^{2} \pi^{2}}{12(c+1)^{3}+(2 n-1)^{2} \pi^{2}(2 c-1)}\right)\right)=\left(n-\frac{1}{2}\right) \pi=\beta_{n, \infty}
$$

então ela tem erro relativo tendendo a zero quando $c$ tende a infinito.

Na Figura 8.18 é mostrado o erro da raiz $\beta_{2}$ e na Figura 8.19 é mostrado o da raiz $\beta_{4}$, ambas da Eq. (1.5), calculadas por estas expressões. A raiz $\beta_{8}$ que seria calculada por estas expressões não consta das figuras porque o trabalho forneceu os pontos de divisão do intervalo correspondente ao domínio da definição de função por partes apenas para as cinco primeiras raízes.

A expressão da Eq. (2.30) para as demais raízes da Eq. (1.7) tem muito boa exatidão não só em relação à tendência dada pela Eq. (2.13):

$$
\lim _{c \rightarrow 0} \frac{\left(n-\frac{1}{2}\right) \pi\left(1+\frac{3}{2(c+2)}\left(\sqrt{1+\frac{16(c-1)(c+2)}{3(2 n-1)^{2} \pi^{2}}}-1\right)\right)-(n-1) \pi}{c} \cong \frac{1}{\beta_{n, 0}}
$$

como também nos seus valores quando $c$ tende a zero:

$$
\lim _{c \rightarrow 0}\left(\left(n-\frac{1}{2}\right) \pi\left(1+\frac{3}{2(c+2)}\left(\sqrt{1+\frac{16(c-1)(c+2)}{3(2 n-1)^{2} \pi^{2}}-1}\right)\right)\right)=\beta_{n, 0}
$$


então tem erro relativos tendendo praticamente a zero quando $c$ tende a zero. Pode-se ver por cálculos análogos aos já mostrados que ela tem erro relativo tendendo a zero quando $c$ tende a infinito.

0 erro das raízes $\beta_{2}, \beta_{4}$ e $\beta_{8}$ da Eq. (1.7) que são calculadas por estas expressões é mostrado na Figura 8.24, Figura 8.25 e Figura 8.26, respectivamente. Pode-se observar nas curvas de erro um comportamento gradativamente sequencial já que, desta vez, o ponto de divisão de domínio da definição de função por partes é sempre $c=6$, o que permite que quaisquer raízes possam ser calculadas, embora perceba-se que o erro esteja aumentando de uma raiz para outra.

Stevens e Luck (1999) apresentaram para todas raízes da Eq. (1.5):

$$
\beta_{n} \cong c_{n}+\frac{\pi}{4}\left(\frac{c-c_{n}}{c+c_{n}}\right)
$$

Para as demais raízes da Eq. (1.6) eles apresentaram:

$$
\beta_{n} \cong d_{n}+\frac{\pi}{4}\left(\frac{c-d_{n}}{c+d_{n}}\right)
$$

E para as demais raízes da Eq. (1.7):

$$
\beta_{n} \cong d_{n}+\frac{\pi}{2}\left(\frac{c-2 / \pi}{c+d_{n}}\right)
$$

onde

$$
\begin{gathered}
c_{n}=(n-3 / 4) \pi \\
d_{n}=(n-1 / 2) \pi
\end{gathered}
$$

As demais raízes da Eq. (1.5) dadas pela expressão da Eq. (2.31) tem valores corretos quando $c$ tende a zero ou infinito, como pode ser facilmente verificado por cálculo de limites, e observado na Figura 8.18, Figura 8.19 e Figura 8.20, para as raízes $\beta_{2}, \beta_{4}$ e $\beta_{8}$, respectivamente.

Porém, esta expressão não satisfaz a tendência da Eq. (2.13):

$$
\lim _{c \rightarrow 0} \frac{c_{n}+\frac{\pi}{4}\left(\frac{c-c_{n}}{c+c_{n}}\right)-(n-1) \pi}{c}=\frac{1}{(0,64 n-0,48) \pi}>\frac{1}{(n-1) \pi}=\frac{1}{\beta_{n, 0}}
$$

O valor incorreto para essa tendência não pode ser visto por estas figuras, mas pode ser visto no gráfico não logarítmico que está na Figura 2.10, através da declividade incorreta das raízes obtidas pela expressão aproximada, em relação aos valores corretos que estão representados por linhas pontilhadas. 
As demais raízes da Eq. (1.6) dadas pela Eq. (2.32) não tem limites corretos quando $c$ tende a zero ou infinito, ou seja, os erros absoluto e relativo não tendem a zero nestes extremos, porém tratase de aproximações razoáveis:

$$
\begin{aligned}
& \lim _{c \rightarrow 0}\left(c_{n}+\frac{\pi}{4}\left(1+\frac{c-\frac{\pi}{4}-c_{n}}{c+\frac{\pi}{4}+c_{n}}\right)\right)=\left(n-\frac{3}{4}\right) \pi \cong \beta_{n, 0} \\
& \lim _{c \rightarrow \infty}\left(c_{n}+\frac{\pi}{4}\left(1+\frac{c-\frac{\pi}{4}-c_{n}}{c+\frac{\pi}{4}+c_{n}}\right)\right)=\left(n-\frac{1}{4}\right) \pi \cong \beta_{n, \infty}
\end{aligned}
$$

como pode ser visto pelas curvas de erro relativo apresentadas na Figura 8.21, Figura 8.22 e Figura 8.23, para as raízes $\beta_{2}, \beta_{4}$ e $\beta_{8}$, respectivamente.

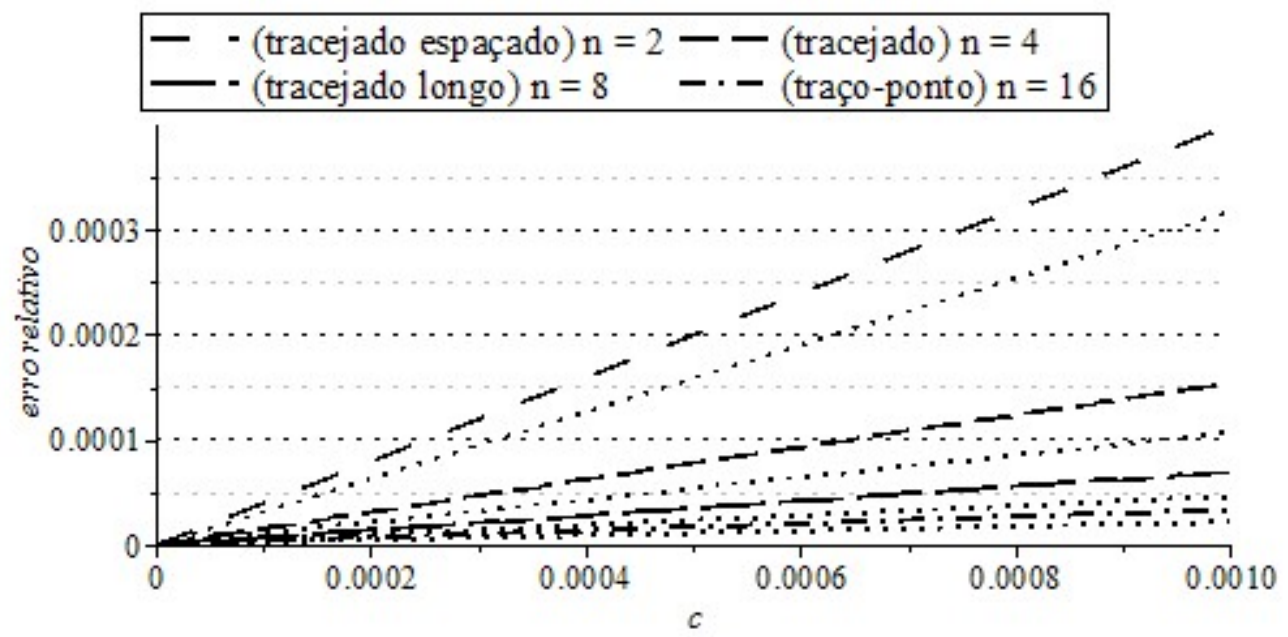

Figura 2.10 Valores aproximados obtidos pela Eq. (2.20) de Stevens e Luck (1999) e valores exatos (representados por linhas pontilhadas) das quatro primeiras raízes da Eq. (1.5)

As demais raízes da Eq. (1.7) dadas pela Eq. (2.33) tem erro absoluto que não tende a zero quando $c$ tende a zero, mas têm aproximação razoável:

$$
\lim _{c \rightarrow 0}\left(\left(n-\frac{1}{2}\right) \pi+\frac{\pi}{2}\left(\frac{c-\frac{2}{\pi}}{c+\left(n-\frac{1}{2}\right) \pi}\right)\right)=\left(n-\frac{1}{2}\right) \pi-\left(n-\frac{1}{2}\right)^{-1} \pi^{-1} \cong \beta_{n, 0}
$$

e tem erro relativo tendendo a zero quando $c$ tende a infinito, o que pode ser visto por cálculos análogos aos já feitos. As raízes obtidas por esta expressão levam às curvas de erro relativo que estão apresentadas na Figura 8.24, Figura 8.25 e Figura 8.26, para as raízes $\beta_{2}$, $\beta_{4}$ e $\beta_{8}$, respectivamente. 
Haji-Sheikh e Beck (2000) propuseram melhoras para as expressões de Stevens e Luck (1999). Eles incluíram a tendência dada pela Eq. (2.12) na expressão da Eq. (2.31), e obtiveram que as raízes da Eq. (1.5) podem ser aproximadas por:

$$
\beta_{n} \cong c_{n}+\frac{\pi}{4} \gamma_{n, 23}\left(n^{-1} \xi_{23}+\left(1-n^{-1}\right) \frac{\tanh \xi_{23}}{\tanh 1}\right)
$$

onde

$$
\begin{gathered}
\gamma_{n, 23}=1-1,04\left(\sqrt{\frac{c+c_{n}-\pi / 4}{c+H}}-\frac{c+\sqrt{H\left(c_{n}-\pi / 4\right)}}{c+H}\right) \\
\xi_{23}=\frac{c-c_{n}}{c+c_{n}} \\
H=0,76+1,22(n-1)
\end{gathered}
$$

e para as demais raízes da Eq. (1.7), eles obtiveram:

$$
\beta_{n} \cong d_{n}+\frac{\pi}{2} \gamma_{n, 13} \xi_{13}
$$

onde

$$
\begin{gathered}
\gamma_{n, 13}=1+\xi_{13}\left(1-\xi_{13}\right)\left(1-\frac{0.85}{n}+\left(0.6-\frac{0.71}{n}\right)\left(1+\xi_{13}\right)\left(0.6+\frac{0.245}{n}-\xi_{13}\right)\right) \\
\xi_{13}=\frac{c}{c+\pi d_{n} / 2} \\
d_{n}=(n-1 / 2) \pi
\end{gathered}
$$

As demais raízes da Eq. (1.5) calculadas pela Eq. (2.34) não satisfazem exatamente a tendência dada pela Eq. (2.13), porém tem erro relativo que tende a zero quando $c$ tende a zero, como poderia ser visto por argumentos e cálculos análogos aos mostrados para a expressão dada na Eq. (2.20); além disso, elas tem valores corretos quando $c$ tende a infinito (Figura 8.18, Figura 8.19 e Figura 8.20, para as raízes $\beta_{2}, \beta_{4}$ e $\beta_{8}$, respectivamente)

As demais raízes da Eq. (1.7) calculadas pela Eq. (2.35) não satisfazem a tendência dada pela Eq. (2.13), e nem mesmo tem erro absoluto que tende a zero quando $c$ tende a zero. Porém, seus erros absoluto e relativo tendem a zero quando $c$ tende a infinito (Figura 8.24, Figura 8.25 e Figura 8.26, para as raízes $\beta_{2}, \beta_{4}$ e $\beta_{8}$, respectivamente). 


\section{NOVAS EXPRESSÕES ITERATIVAS PARA AS RAÍZES DAS EQS. (1.5), (1.6) E}

O trabalho feito para se desenvolver uma função iterativa para calcular eficientemente as raízes das Eqs. (1.5), (1.6) e (1.7), acabou conduzindo a uma nova família de métodos que pode ser utilizada para qualquer equação. Esse método é baseado na aproximação de Padé, e a família de métodos obtida incluiu várias funções iterativas clássicas, inclusive a família de métodos de Householder.

\subsection{Funções Iterativas obtidas com o Polinômio de Taylor}

A aproximação de uma função $f(x)$ por seu polinômio de Taylor de grau $m$ na vizinhança de $x=a$ é dada por:

$$
f(x) \approx c_{0}+c_{1}(x-a)+c_{2}(x-a)^{2}+\cdots+c_{m}(x-a)^{m}
$$

onde os coeficientes $c_{0}, c_{1}, \ldots, c_{m}$ são obtidos através de:

$$
\left.\frac{d^{i}}{d x^{i}} f(x)\right|_{x=a}=\left.\frac{d^{i}}{d x^{i}}\left(c_{0}+c_{1}(x-a)+\cdots+c_{m}(x-a)^{m}\right)\right|_{x=a}, i=0,1,2, \ldots, m
$$

o que leva a:

$$
c_{i}=\frac{f^{(i)}(a)}{i !}, i=0,1,2, \ldots, m
$$

Duas funções iterativas clássicas para as raízes de equações podem ser obtidas através da aproximação por polinômios de Taylor de graus 1 e 2 através de um procedimento muito conhecido. 0 ponto $a$ é substituído pela estimativa $x_{k}$, a variável $x$ é substituída pela estimativa seguinte $x_{k+1}$, é assumido que $f\left(x_{k+1}\right)=0$, e então:

$$
f\left(x_{k}\right)+f^{\prime}\left(x_{k}\right)\left(x_{k+1}-x_{k}\right)+\cdots+\frac{f^{(m)}\left(x_{k}\right)}{m !}\left(x_{k+1}-x_{k}\right)^{m}=0
$$

Para $m=1$, esta equação resulta em:

$$
x_{k+1}=x_{k}-\frac{f\left(x_{k}\right)}{f^{\prime}\left(x_{k}\right)}
$$


o qual é conhecida como método de Newton-Raphson de busca de raízes.

Neste texto, uma simplificação conveniente das notações de uma função $f$ e suas derivadas através da supressão de $\left(x_{k}\right)$ será usada para facilitar a comparação entre os vários métodos. Então, o método de Newton-Raphson será expresso por:

$$
x_{k+1}=x_{k}-\frac{f}{f^{\prime}}
$$

Para $m=2$, a Eq. (3.4) resulta em:

$$
x_{k+1}=x_{k}-\frac{f}{f^{\prime}} \frac{2}{1+\sqrt{1-f \frac{2 f^{\prime \prime}}{f^{\prime 2}}}}
$$

que é conhecida como método de Euler (AMAT; BUSQUIER; GUTIÉRREZ; 2003, KANWAR; SINGH; BAKSHI, 2008). Uma das raízes correspondentes a equação quadrática foi desconsiderada devido ao fato de que $x_{k}$ deve tender a $x_{k+1}$ quando $f\left(x_{k}\right)$ tende a zero (como pode ser visto pela regra de L'Hôpital).

O polinômio de Taylor pode ser considerado como um caso particular da aproximação de Padé (BAKER, 1996). Da mesma maneira que os métodos de busca de raízes de Eqs. (3.5) e (3.7) são obtidos por um procedimento que utiliza o polinômio de Taylor, obteremos outros métodos utilizando a aproximação de Padé (MERZ, 1968, NOUREIN, 1976, CLAESSENS; LOIZOU; WUYTACK, 1977, FIELD, 1990, SAKURAI; TORII; SUGIURA, 1991; LI ET AL., 2012).

\subsection{Funções Iterativas Com Derivadas Segundas}

A aproximação de Padé de ordem $[m / n]$ na vizinhança de um ponto $x=a$ de uma função $f(x)$, é definida por:

$$
f(x) \approx \frac{a_{0}+a_{1}(x-a)+a_{2}(x-a)^{2}+\cdots+a_{m}(x-a)^{m}}{1+b_{1}(x-a)+b_{2}(x-a)^{2}+\cdots+b_{n}(x-a)^{n}}
$$

onde os coeficientes $a_{0}, a_{1}, \ldots, a_{m}, b_{1}, b_{2}, \ldots, b_{n}$ são dados por:

$$
\left.\frac{d^{i}}{d x^{i}} f(x)\right|_{x=a}=\left.\frac{d^{i}}{d x^{i}} \frac{a_{0}+a_{1}(x-a)+\cdots+a_{m}(x-a)^{m}}{1+b_{1}(x-a)+\cdots+b_{n}(x-a)^{n}}\right|_{x=a}, i=0,1,2, \ldots, m+n
$$

A diferença para o polinômio de Taylor é que estes coeficientes não têm expressões simples. 
A substituição de $m$ e $n$ por 1 nas Eqs. (3.8) e (3.9) resultam na aproximação de Padé de ordem $[1 / 1]:$

$$
f(x) \approx \frac{f(a)+\left(f^{\prime}(a)-\frac{f(a) f^{\prime \prime}(a)}{2 f^{\prime}(a)}\right)(x-a)}{1-\frac{f^{\prime \prime}(a)}{2 f^{\prime}(a)}(x-a)}
$$

que com as mesmas substituições feitas no polinômio de Taylor para se obter os métodos iterativos já mostrados resulta em:

$$
\frac{f+\left(f^{\prime}-\frac{f f^{\prime \prime}}{2 f^{\prime}}\right)\left(x_{k+1}-x_{k}\right)}{1-\frac{f^{\prime \prime}}{2 f^{\prime}}\left(x_{k+1}-x_{k}\right)}=0
$$

que imediatamente leva a:

$$
x_{k+1}=x_{k}-\frac{f}{f^{\prime}} \frac{1}{1-f \frac{f^{\prime \prime}}{2 f^{\prime 2}}}
$$

conhecida como método de Halley (AMAT; BUSQUIER; GUTIÉRREZ; 2003, KANWAR; SINGH; BAKSHI, 2008).

Três outras funções iterativas clássicas podem ser obtidas a partir da Eq. (3.12). A primeira é obtida através de:

$$
x_{k+1}=x_{k}-\frac{f}{f^{\prime}} \frac{1}{1-f \frac{f^{\prime \prime}}{2 f^{\prime 2}}} \frac{1-f \frac{f^{\prime \prime}}{2 f^{\prime 2}}}{1-f \frac{f^{\prime \prime}}{2 f^{\prime 2}}}=x_{k}-\frac{f}{f^{\prime}} \frac{1-f \frac{f^{\prime \prime}}{2 f^{\prime 2}}}{1-2 f \frac{f^{\prime \prime}}{2 f^{\prime 2}}+f^{2}\left(\frac{f^{\prime \prime}}{2 f^{\prime 2}}\right)^{2}}
$$

onde $f^{2}$ tem valor bem menor do que os demais termos por ser o quadrado de um valor já baixo $f\left(x_{k}\right)$. Então:

$$
x_{k+1}=x_{k}-\frac{f}{f^{\prime}} \frac{1-f \frac{f^{\prime \prime}}{2 f^{\prime 2}}}{1-2 f \frac{f^{\prime \prime}}{2 f^{\prime 2}}}=x_{k}-\frac{f}{f^{\prime}} \frac{2 f^{\prime 2}-f f^{\prime \prime}}{2 f^{\prime 2}-2 f f^{\prime \prime}}
$$

o que leva a:

$$
x_{k+1}=x_{k}-\frac{f}{f^{\prime}}\left(1+f \frac{f^{\prime \prime}}{2 f^{\prime 2}-2 f f^{\prime \prime}}\right)
$$

conhecida como método de Super-Halley (AMAT; BUSQUIER; GUTIÉRREZ; 2003, KANWAR; SINGH; BAKSHI, 2008). 
Utilizando $(1-\alpha)^{-1} \cong 1+\alpha$ para $|\alpha| \ll 1$ na Eq. (3.12), ou negligenciando $f f^{\prime \prime}$ na Eq. (3.13), obtém-se:

$$
x_{k+1}=x_{k}-\frac{f}{f^{\prime}}\left(1+f \frac{f^{\prime \prime}}{2 f^{\prime 2}}\right)
$$

conhecida como método de Chebyshev (AMAT; BUSQUIER; GUTIÉRREZ; 2003, KANWAR; SINGH; BAKSHI, 2008).

Utilizando $(1-\alpha)^{1 / 2} \cong 1-\alpha / 2$ para $|\alpha| \ll 1$ na Eq. (3.12), obtém-se:

$$
x_{k+1}=x_{k}-\frac{f}{f^{\prime}} \frac{1}{\sqrt{1-f \frac{f^{\prime \prime}}{f^{\prime 2}}}}
$$

conhecida como iteração da raiz quadrada de Ostrowski (AMAT; BUSQUIER; GUTIÉRREZ; 2003, KANWAR; SINGH; BAKSHI, 2008).

Utilizando novamente $(1-\alpha)^{1 / 2} \cong 1-\alpha / 2$ para $|\alpha| \ll 1$, também é possível se obter as Eqs. (3.7) e (3.12), uma a partir da outra. Por fim, usando a tendência de que $f$ se aproxima de zero, podese obter o método de Newton-Raphson a partir de qualquer um dos outros acima.

\subsection{Funções Iterativas Com Derivadas Terceiras}

Substituindo $m$ por 1 e $n$ por 2 nas Eqs. (3.8) e (3.9), a aproximação de Padé de ordem [1/2] é obtida, e com a substituição acima descrita, ela resulta em:

$$
\frac{f+\left(f^{\prime}+\frac{3 f f^{\prime} f^{\prime \prime}-f^{2} f^{\prime \prime \prime}}{3 f f^{\prime \prime}-6 f^{\prime 2}}\right)\left(x_{k+1}-x_{k}\right)}{1-\frac{3 f^{\prime} f^{\prime \prime}-f f^{\prime \prime \prime}}{3 f f^{\prime \prime}-6 f^{\prime 2}}\left(x_{k+1}-x_{k}\right)+\frac{f^{\prime} f^{\prime \prime \prime}-f^{\prime \prime 2}}{3 f f^{\prime \prime}-6 f^{\prime 2}}\left(x_{k+1}-x_{k}\right)^{2}}=0
$$

e, portanto:

$$
x_{k+1}=x_{k}-\frac{f}{f^{\prime}} \frac{1}{1-f \frac{f^{\prime \prime}}{2 f^{\prime 2}} \frac{1-f \frac{f^{\prime \prime \prime}}{3 f^{\prime} f^{\prime \prime}}}{1-f \frac{f^{\prime \prime}}{2 f^{\prime 2}}}}
$$

que é um dos casos do método de Householder (1970). Observe-se que com a aproximação de $f$ para zero, a Eq. (3.12) é obtida da Eq. (3.17) e, portanto, todas outras apresentadas acima são também obtidas desta Eq. (3.17). 
Utilizando $(1-\alpha)^{-1} \cong 1+\alpha$ para $|\alpha| \ll 1$ na Eq. (3.17):

$$
\begin{aligned}
& x_{k+1}=x_{k}-\frac{f}{f^{\prime}} \frac{1}{1-f \frac{f^{\prime \prime}}{2 f^{\prime 2}} \frac{1}{1-f \frac{f^{\prime \prime}}{2 f^{\prime 2}}} \frac{1}{1+f \frac{f^{\prime \prime \prime}}{3 f^{\prime} f^{\prime \prime}}}} \\
& =x_{k}-\frac{f}{f^{\prime}} \frac{1}{1-f \frac{f^{\prime \prime}}{2 f^{\prime 2}} \frac{1}{1-f \frac{f^{\prime \prime}}{2 f^{\prime 2}}+f \frac{f^{\prime \prime \prime}}{3 f^{\prime} f^{\prime \prime}}+f^{2} \frac{f^{\prime \prime \prime}}{6 f^{\prime 3}}}}
\end{aligned}
$$

e negligenciando-se o termo $\operatorname{com} f^{2}$, obtém-se:

$$
x_{k+1}=x_{k}-\frac{f}{f^{\prime}} \frac{1}{1-f \frac{f^{\prime \prime}}{2 f^{\prime 2}} \frac{1}{1-f\left(\frac{f^{\prime \prime}}{2 f^{\prime 2}}-\frac{f^{\prime \prime \prime}}{3 f^{\prime} f^{\prime \prime}}\right)}}
$$

que é a função (5a) da compilação de funções iterativas de um ponto de Traub (1964).

Utilizando novamente $(1-\alpha)^{-1} \cong 1+\alpha$ para $|\alpha| \ll 1$ na Eq. (3.18), obtém-se:

$$
x_{k+1}=x_{k}-\frac{f}{f^{\prime}} \frac{1}{1-f \frac{f^{\prime \prime}}{2 f^{\prime 2}}\left(1+f\left(\frac{f^{\prime \prime}}{2 f^{\prime 2}}-\frac{f^{\prime \prime \prime}}{3 f^{\prime} f^{\prime \prime}}\right)\right)}
$$

que é a função (6a) da compilação de funções iterativas de um ponto de Traub (1964).

Utilizando, além dessa aproximação, também $(1-\alpha)^{-1} \cong 1+\alpha+\alpha^{2}$ para $|\alpha| \ll 1$, na Eq. (3.18), obtém-se:

$$
\begin{aligned}
\left.x_{k+1}=x_{k}-\frac{f}{f^{\prime}}\left(1+\frac{f \frac{f^{\prime \prime}}{2 f^{\prime 2}}}{1-f\left(\frac{f^{\prime \prime}}{2 f^{\prime 2}}-\frac{f^{\prime \prime \prime}}{3 f^{\prime} f^{\prime \prime}}\right)}+\left(\frac{f \frac{f^{\prime \prime}}{2 f^{\prime 2}}}{1-f\left(\frac{f^{\prime \prime}}{2 f^{\prime 2}}-\frac{f^{\prime \prime \prime}}{3 f^{\prime} f^{\prime \prime}}\right)}\right)\right)^{2}\right) \\
=x_{k}-\frac{f}{f^{\prime}}\left(1+f \frac{f^{\prime \prime}}{2 f^{\prime 2}}\left(1+f\left(\frac{f^{\prime \prime}}{2 f^{\prime 2}}-\frac{f^{\prime \prime \prime}}{3 f^{\prime} f^{\prime \prime}}\right)\right)+\left(f \frac{f^{\prime \prime}}{2 f^{\prime 2}}\right)^{2}\right)
\end{aligned}
$$

e, então:

$$
x_{k+1}=x_{k}-\frac{f}{f^{\prime}}\left(1+f \frac{f^{\prime \prime}}{2 f^{\prime 2}}\left(1+f\left(\frac{f^{\prime \prime}}{f^{\prime 2}}-\frac{f^{\prime \prime \prime}}{3 f^{\prime} f^{\prime \prime}}\right)\right)\right)
$$

que é a função (3a) da compilação de funções iterativas de um ponto de Traub (1964), e que observamos também ter sido redescoberta por Pakdemirli et al. (2007). 
Finalmente, utilizando $(1-\alpha)^{1 / 2} \cong 1-\alpha / 2$ para $|\alpha| \ll 1$ na Eq. (3.17), e negligenciando-se um dos termos que contém $f$, obtém-se:

$$
x_{k+1}=x_{k}-\frac{f}{f^{\prime}} \frac{2}{1+\sqrt{1-f \frac{2 f^{\prime \prime}}{f^{\prime 2}}\left(1-f \frac{f^{\prime \prime \prime}}{3 f^{\prime} f^{\prime \prime}}\right)}}
$$

que é a função (8a) da compilação de funções iterativas de um ponto de Traub (1964). A lista completa dessa compilação é constituída de oito funções: Eqs. (3.6), (3.7), (3.12), (3.14), (3.18), (3.19), (3.20) e (3.21).

Substituindo $m=2$ e $n=1$ nas Eqs. (3.8) e (3.9), a aproximação de Padé de ordem [2/1] é obtida, e com as substituições prévias, ela resulta em:

$$
\frac{f+\left(f^{\prime}-\frac{f f^{\prime \prime \prime}}{3 f^{\prime \prime}}\right)\left(x_{k+1}-x_{k}\right)+\left(\frac{f^{\prime \prime}}{2}-\frac{f^{\prime} f^{\prime \prime \prime}}{3 f^{\prime \prime}}\right)\left(x_{k+1}-x_{k}\right)^{2}}{1-\frac{f^{\prime \prime \prime}}{3 f^{\prime \prime}}\left(x_{k+1}-x_{k}\right)}=0
$$

a partir da qual uma nova função iterativa é obtida:

$$
x_{k+1}=x_{k}-\frac{f}{B} \frac{1}{1+\sqrt{1-\frac{A}{B^{2}} f}}
$$

onde

$$
A=\frac{f^{\prime \prime}}{2}-\frac{f^{\prime} f^{\prime \prime \prime}}{3 f^{\prime \prime}} \text { e } B=\frac{f^{\prime}}{2}-\frac{f f^{\prime \prime \prime}}{6 f^{\prime \prime}}
$$

e uma das raízes da correspondente à equação quadrática foi desconsiderada devido ao fato de que $x_{k+1}-x_{k}$ não tenderia a zero quando $f\left(x_{k}\right)$ tendesse a zero.

\subsection{Funções Iterativas Com Derivadas de Ordem Maior do que Três}

É possível se obter mais funções com derivadas de ordem maior do que três, através de valores maiores de $m$ e $n$, mas aqui somente o caso onde $m=1$ será discutido, que é o mais simples, porque é mais fácil se tratar de funções lineares.

Então, substituindo $m$ por 1 na Eq. (3.8), obtém-se:

$$
f(x) \cong \frac{a_{0}+a_{1}(x-a)}{1+b_{1}(x-a)+b_{2}(x-a)^{2}+\cdots+b_{n}(x-a)^{n}}
$$


que com as prévias substituições, resulta em

$$
x_{k+1}=x_{k}-\frac{a_{0}}{a_{1}}
$$

Da Eq. (3.25), para $p \geq n$, obtém-se:

$$
\left(\frac{1}{f}\right)^{(p)}(x) \approx(-1)^{p} p ! \frac{a_{1}^{p}-a_{0} a_{1}^{p-1} b_{1}+a_{0}^{2} a_{1}^{p-2} b_{2}+\cdots+(-1)^{n} a_{0}^{n} a_{1}^{p-n} b_{n}}{\left(a_{0}+a_{1}(x-a)\right)^{p+1}}
$$

que resulta em:

$$
\left(\frac{1}{f}\right)^{(p)}(a) \approx(-1)^{p} p ! \frac{a_{1}^{p}-a_{0} a_{1}^{p-1} b_{1}+a_{0}^{2} a_{1}^{p-2} b_{2}+\cdots+(-1)^{n} a_{0}^{n} a_{1}^{p-n} b_{n}}{a_{0}^{p+1}}
$$

e, portanto:

$$
\left(\frac{1}{f}\right)^{(n+1)}(a) \approx-(n+1) \frac{a_{1}}{a_{0}}\left(\frac{1}{f}\right)^{(n)}(a)
$$

A substituição de $a_{0} / a_{1}$ da Eq. (3.26) por seu valor dado pela Eq. (3.29) resulta em:

$$
x_{k+1}=x_{k}+(n+1) \frac{(1 / f)^{(n)}}{(1 / f)^{(n+1)}}
$$

a qual é a classe de Métodos de Householder (1970) e tem a Eq. (3.17) como caso particular correspondente a $n=2$.

\subsection{Organização das Funções Iterativas}

Como $f$ pode representar uma quantidade física com uma unidade diferente da unidade da variável independente $x$, pelo Teorema dos $\Pi$ de Buckingham, conclui-se que as funções iterativas que incluem $x, f, f^{\prime}, f^{\prime \prime}$ e $f^{\prime \prime \prime}$, podem ser reescritas com $5-2=3$ variáveis adimensionais.

Observando as funções iterativas apresentadas, escolhemos como parâmetros П:

$$
f_{1}=\frac{f}{f^{\prime}}, f_{2}=\frac{f f^{\prime \prime}}{2 f^{\prime 2}} \text { e } f_{3}=\frac{f f^{\prime \prime \prime}}{3 f^{\prime} f^{\prime \prime}}
$$

e a Tabela 3.1 reapresenta as funções iterativas simplificadas pela utilização desses parâmetros, e ordenadas quanto as suas complexidades.

Por esta tabela é possível se ver facilmente a equivalência das funções iterativas que utilizam derivadas até a mesma ordem, e também como as que utilizam menor ordem podem ser obtidas a 
partir das que utilizam maior ordem, conforme as aproximações elementares anteriormente apresentadas. Essa tabela poderia conter mais nomes de métodos, se fossem mencionados os vários nomes que são atribuídos aos mesmos métodos na literatura (PETKOVIĆ; HERCEG, 1999, PETKOVIĆ; PETKOVIĆ; HERCEG, 2010).

Tabela 3.1 Compilação de funções iterativas de um ponto

\begin{tabular}{|c|c|c|}
\hline Eq. & Método & Função Iterativa \\
\hline$(3.6)$ & Newton-Raphson & $x_{k+1}=x_{k}-f_{1}$ \\
\hline$(3.14)$ & Chebyshev & $x_{k+1}=x_{k}-f_{1}\left(1+f_{2}\right)$ \\
\hline$(3.12)$ & Halley & $x_{k+1}=x_{k}-f_{1} \frac{1}{1-f_{2}}$ \\
\hline$(3.15)$ & Ostrowski & $x_{k+1}=x_{k}-f_{1} \frac{1}{\sqrt{1-2 f_{2}}}$ \\
\hline$(3.13)$ & Super-Halley & $x_{k+1}=x_{k}-f_{1} \frac{1-f_{2}}{1-2 f_{2}}$ \\
\hline$(3.7)$ & Euler & $x_{k+1}=x_{k}-f_{1} \frac{2}{1+\sqrt{1-4 f_{2}}}$ \\
\hline$(3.20)$ & Traub (3a) & $x_{k+1}=x_{k}-f_{1}\left(1+f_{2}\left(1+2 f_{2}-f_{3}\right)\right)$ \\
\hline (3.19) & Traub (6a) & $x_{k+1}=x_{k}-f_{1} \frac{1}{1-f_{2}\left(1+f_{2}-f_{3}\right)}$ \\
\hline$(3.18)$ & Traub (5a) & $x_{k+1}=x_{k}-f_{1} \frac{1}{1-f_{2} \frac{1}{1-f_{2}+f_{3}}}$ \\
\hline$(3.17)$ & Householder & $x_{k+1}=x_{k}-f_{1} \frac{1}{1-f_{2} \frac{1-f_{3}}{1-f_{2}}}$ \\
\hline$(3.21)$ & Traub (8a) & $x_{k+1}=x_{k}-f_{1} \frac{}{1+\sqrt{1-4 f_{2}\left(1-f_{3}\right)}}$ \\
\hline$(3.23)$ & Presente trabalho & $x_{k+1}=x_{k}-\frac{J 1}{1-f_{3}} \frac{2}{1+\sqrt{1-4 \frac{f_{2}-f_{3}}{\left(1-f_{3}\right)^{2}}}}$ \\
\hline
\end{tabular}

É possível se obter novas funções iterativas observando as lacunas lógicas desta tabela. Por exemplo:

$$
x_{k+1}=x_{k}-f_{1} \sqrt{1+2 f_{2}}
$$


pode ser obtida por várias maneiras a partir das funções tabeladas. É fácil provar que ela tem ordem de convergência 3, e isso poderá ser discutido em próximos trabalhos.

\subsection{Comparação de Resultados}

Para se escolher um grupo aleatório de funções, utilizou-se os exercícios sobre método de Newton-Raphson de Burden, Faires e Burden (2015), e o resultado está na Tabela 3.2. É claro que esse grupo não pode ser considerado como uma amostra representativa das funções para as quais pode-se buscar raízes e observou-se resultados bastante diferentes para outras funções testadas.

Tabela 3.2 Cologaritmo do módulo do erro relativo máximo das raízes de várias equações após 3 iterações

\begin{tabular}{|c|c|c|c|c|c|c|c|c|c|c|c|c|c|c|}
\hline Equação & 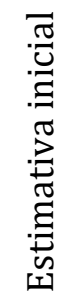 & $\begin{array}{l}\stackrel{N}{\pi} \\
\widetilde{\pi}\end{array}$ & 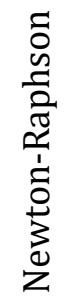 & 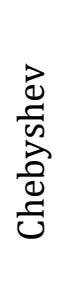 & $\frac{\text { d) }}{\stackrel{\widetilde{\pi}}{\widetilde{I}}}$ & \begin{tabular}{l}
$\frac{\bar{y}}{w}$ \\
$\vdots$ \\
0 \\
\multirow{2}{5}{} \\
0
\end{tabular} & 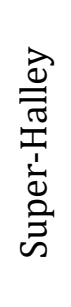 & 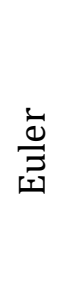 & 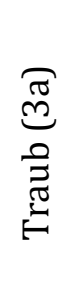 & 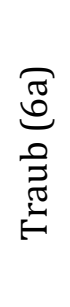 & 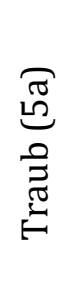 & 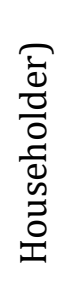 & 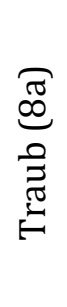 & 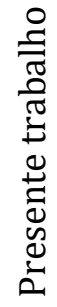 \\
\hline$x^{3}-2 x^{2}-5=0$ & 4 & 2,69 & 3 & 8 & 10 & 15 & 25 & 8 & 16 & 21 & 24 & 27 & 8 & 35 \\
\hline$x^{3}+3 x^{2}-1=0$ & -3 & $-2,9$ & 9 & 27 & 31 & 37 & 38 & 37 & 58 & 67 & 72 & 75 & 37 & 94 \\
\hline$x-\cos x=0$ & 0 & 0,74 & 4 & 8 & 14 & 17 & 22 & 24 & 15 & 26 & 41 & 40 & 24 & 41 \\
\hline$x-0,8-0,2$ & 0.5 & 0,96 & 10 & 33 & 31 & 30 & 29 & 29 & 59 & 62 & 53 & 63 & 29 & 55 \\
\hline$e^{x}+2^{-x}+2 \cos x-6=0$ & 2 & 1,83 & 7 & 22 & 27 & 41 & 28 & 27 & 49 & 60 & 64 & 71 & 27 & 75 \\
\hline $\ln (x-1)+\cos (x-1)=0$ & 1.3 & 1,40 & 7 & 21 & 45 & 25 & 20 & 19 & 48 & 62 & 62 & 62 & 19 & 62 \\
\hline $2 x \cos 2 x-(x-2)^{2}=0$ & 2 & 2,37 & 7 & 4 & 11 & 14 & 20 & 18 & - & 17 & 28 & 31 & 18 & 33 \\
\hline $2 x \cos 2 x-(x-2)^{2}=0$ & 4 & 3,72 & 8 & 17 & 18 & 19 & 19 & 19 & 40 & 45 & 22 & 48 & 19 & 21 \\
\hline$(x-2)^{2}-\ln x=0$ & 1 & 1,41 & 5 & 15 & 19 & 25 & 26 & 23 & 32 & 39 & 41 & 43 & 23 & 52 \\
\hline$(x-2)^{2}-\ln x=0$ & 4 & 3,06 & 4 & 10 & 12 & 15 & 22 & 32 & 20 & 25 & 29 & 29 & 32 & 67 \\
\hline$e^{x}-3 x^{2}=0$ & 1 & 0,91 & 10 & 30 & 33 & 35 & 39 & 39 & 67 & 74 & 83 & 79 & 39 & 90 \\
\hline$e^{x}-3 x^{2}=0$ & 5 & 3,73 & 2 & 5 & 7 & 18 & 7 & 4 & 9 & 14 & 16 & 20 & 4 & 14 \\
\hline $\sin x-e^{-x}=0$ & 0 & 0,59 & 5 & 16 & 19 & 21 & 26 & 29 & 34 & 38 & 42 & 42 & 29 & 54 \\
\hline $\sin x-e^{-x}=0$ & 3 & 3,10 & 17 & 37 & 37 & 37 & 38 & 38 & 89 & 95 & 75 & 99 & 38 & 75 \\
\hline $\sin x-e^{-x}=0$ & 6 & 6,29 & 17 & 25 & 25 & 26 & 26 & 26 & 66 & 72 & 43 & 78 & 26 & 42 \\
\hline
\end{tabular}

Os métodos desta tabela estão na mesma ordem da Tabela 3.1, onde se observa que os resultados de um método podem ou não ser melhores do que os de outro, dependendo de sinais das funções e de suas derivadas, e mesmo da raiz que se busca.

Então, o importante é entender que a ordem de convergência de um método é definida matematicamente para um número infinito de iterações e, quando o objetivo é o de se procurar um 
método que leve a uma determinada exatidão com um número mínimo de iterações, deve-se testar os métodos disponíveis, para a determinada equação, raiz que se busca, e estimativa inicial (KUNG; TRAUB, 1974, GONNET, 2001, NETA; SCOTT; CHUN, 2012). Por isso, escolheu-se, arbitrariamente 2 ou 3 iterações para as comparações.

Também é importante saber que todas funções iterativas que utilizam mesmo número de derivadas têm custo computacional praticamente igual quando as funções cujas raízes se procuram são transcendentes. Isso ocorre devido ao custo computacional ser muito maior para o cálculo de expressões transcendentes do que para as operações algébricas realizadas sobre elas. Então, o novo método apresentado neste trabalho tem custo computacional semelhante aos dos outros cinco métodos discutidos que utilizam até derivada terceira.

\subsection{Aplicação para as Raízes das Eqs. (1.5), (1.6) e (1.7)}

Expressões com erro máximo menor para pontos iniciais não implicam necessariamente em melhor convergência para todas funções iterativas porque cada uma é mais sensível ao erro de determinado intervalo de $c$.

Apesar de que o interesse sobre as Eqs. (1.5), (1.6) e (1.7) seja muito maior nas primeiras raízes, mais raízes serão incluídas nos resultados, para que eles fiquem mais completos, já que isso não implicará em espaço adicional ou esforço relevante.

Para a aproximação inicial da primeira raiz $\beta_{1}$, será utilizada a expressão da Eq. (2.19) de Yovanovich (1996), que pode ser escrita como:

$$
\beta_{1,0}=p\left(1+q c^{r / 2}\right)^{1 / r}
$$

onde $p=1,57,2,40,3,14, q=2,62,3,29,4,02$ e $r=2,14,2,25,2,34$ para as Eqs. (1.5), (1.6) e (1.7), respectivamente.

Para as aproximações iniciais das demais raízes $\beta_{l} \operatorname{com} l=2,3, \ldots$, serão utilizadas as expressões das Eqs. (2.31), (2.32) e (2.33) de Stevens e Luck (1999), que podem ser sintetizadas numa única expressão:

$$
\beta_{l, 0}=\pi(l-p)+\frac{c-q}{\frac{2 c}{\pi}+2 l-r}
$$

onde $p=1,0,75,0,5, q=0,0,2 / \pi$ e $r=1,5,1,1$ para as Eqs. (1.5), (1.6) e (1.7), respectivamente.

Até mesmo a forma das funções influencia na convergência dos métodos. Por exemplo, as duas funções que levam as raízes da Eq. (1.5): 


$$
\begin{gathered}
f\left(\beta_{n}\right)=\beta_{n} \tan \beta_{n}-c \\
f\left(\beta_{n}\right)=\beta_{n} \operatorname{sen} \beta_{n}-c \cos \beta_{n}
\end{gathered}
$$

levam a velocidades iniciais de convergência diferentes em um mesmo método. Serão utilizadas as seguintes opções:

$$
f\left(\beta_{n}\right)=\beta_{n} \tan \beta_{n}-c
$$

para a Eq. (1.5),

$$
f\left(\beta_{n}\right)=\beta_{n} \frac{\mathrm{J}_{1}\left(\beta_{n}\right)}{\mathrm{J}_{0}\left(\beta_{n}\right)}-c
$$

para a Eq. (1.6), e

$$
f\left(\beta_{n}\right)=\beta_{n} \cot \beta_{n}+c-1
$$

para a Eq. (1.7), já que os pontos de descontinuidade que surgem nessas funções não interferem na convergência, e essas formas levam a funções iterativas mais simples. Uma comparação entre os resultados das funções iterativas foi feita através do erro relativo máximo que se obtém para cada raiz na segunda iteração conforme se varia o $c$ (Tabela 3.3, Tabela 3.4 e Tabela 3.5 para as Eqs. (1.5), (1.6) e (1.7), respectivamente). Os resultados não apresentados correspondem a métodos que não convergiram para alguma faixa de valores de $c$ como, por exemplo, o caso já citado de Stevens e Luck da Eq. (2.3).

Tabela 3.3 Erro relativo máximo de raízes da Eq. (1.5) após 2 iterações

\begin{tabular}{cccccccccc}
\hline \multirow{2}{*}{ Método } & \multirow{2}{*}{ Eq. } & \multicolumn{7}{c}{ Raiz } \\
\cline { 3 - 9 } & & $1^{\mathrm{a}}$ & $2^{\mathrm{a}}$ & $4^{\mathrm{a}}$ & $8^{\mathrm{a}}$ & $16^{\mathrm{a}}$ & $32^{\mathrm{a}}$ & $64^{\mathrm{a}}$ & $12^{\mathrm{a}}$ \\
\hline Stevens e Luck simp. & $(2.6)$ & $6 \cdot 10^{-12}$ & $6 \cdot 10^{-9}$ & $5 \cdot 10^{-10}$ & $1 \cdot 10^{-11}$ & $7 \cdot 10^{-13}$ & $4 \cdot 10^{-13}$ & $2 \cdot 10^{-13}$ & $7 \cdot 10^{-14}$ \\
Stevens e Luck & $(2.3)$ & $5 \cdot 10^{-27}$ & $4 \cdot 10^{-16}$ & $8 \cdot 10^{-15}$ & $1 \cdot 10^{-14}$ & $8 \cdot 10^{-15}$ & $5 \cdot 10^{-15}$ & $3 \cdot 10^{-15}$ & $1 \cdot 10^{-15}$ \\
Newton-Raphson & $(3.6)$ & - & $6 \cdot 10^{-5}$ & $1 \cdot 10^{-4}$ & $1 \cdot 10^{-4}$ & $6 \cdot 10^{-5}$ & $3 \cdot 10^{-5}$ & $2 \cdot 10^{-5}$ & $8 \cdot 10^{-6}$ \\
Chebyshev & $(3.14)$ & $8 \cdot 10^{-10}$ & $8 \cdot 10^{-8}$ & $1 \cdot 10^{-6}$ & $2 \cdot 10^{-6}$ & $1 \cdot 10^{-6}$ & $8 \cdot 10^{-7}$ & $4 \cdot 10^{-7}$ & $2 \cdot 10^{-7}$ \\
Halley & $(3.12)$ & $4 \cdot 10^{-26}$ & $2 \cdot 10^{-16}$ & $5 \cdot 10^{-15}$ & $8 \cdot 10^{-15}$ & $7 \cdot 10^{-15}$ & $5 \cdot 10^{-15}$ & $3 \cdot 10^{-15}$ & $1 \cdot 10^{-15}$ \\
Ostrowski & $(3.15)$ & - & $1 \cdot 10^{-9}$ & $1 \cdot 10^{-8}$ & $1 \cdot 10^{-8}$ & $1 \cdot 10^{-8}$ & $6 \cdot 10^{-9}$ & $3 \cdot 10^{-9}$ & $2 \cdot 10^{-9}$ \\
Super-Halley & $(3.13)$ & - & $2 \cdot 10^{-8}$ & $2 \cdot 10^{-7}$ & $3 \cdot 10^{-7}$ & $2 \cdot 10^{-7}$ & $1 \cdot 10^{-7}$ & $6 \cdot 10^{-8}$ & $3 \cdot 10^{-8}$ \\
Euler & $(3.7)$ & - & $1 \cdot 10^{-8}$ & $1 \cdot 10^{-7}$ & $1 \cdot 10^{-7}$ & $1 \cdot 10^{-7}$ & $6 \cdot 10^{-8}$ & $3 \cdot 10^{-8}$ & $2 \cdot 10^{-8}$ \\
Traub (3a) & $(3.20)$ & - & $1 \cdot 10^{-11}$ & $3 \cdot 10^{-9}$ & $1 \cdot 10^{-8}$ & $1 \cdot 10^{-8}$ & $7 \cdot 10^{-9}$ & $4 \cdot 10^{-9}$ & $2 \cdot 10^{-9}$ \\
Traub (6a) & $(3.19)$ & $5 \cdot 10^{-4}$ & $5 \cdot 10^{-24}$ & $2 \cdot 10^{-21}$ & $9 \cdot 10^{-21}$ & $1 \cdot 10^{-20}$ & $8 \cdot 10^{-21}$ & $5 \cdot 10^{-21}$ & $3 \cdot 10^{-21}$ \\
Traub (5a) & $(3.18)$ & - & $5 \cdot 10^{-24}$ & $3 \cdot 10^{-21}$ & $1 \cdot 10^{-20}$ & $1 \cdot 10^{-20}$ & $9 \cdot 10^{-21}$ & $6 \cdot 10^{-21}$ & $3 \cdot 10^{-21}$ \\
Householder & $(3.17)$ & $6 \cdot 10^{-46}$ & $4 \cdot 10^{-30}$ & $7 \cdot 10^{-30}$ & $7 \cdot 10^{-32}$ & $2 \cdot 10^{-35}$ & $1 \cdot 10^{-33}$ & $1 \cdot 10^{-33}$ & $6 \cdot 10^{-34}$ \\
Traub (8a) & $(3.21)$ & $1 \cdot 10^{-10}$ & $3 \cdot 10^{-24}$ & $6 \cdot 10^{-22}$ & $2 \cdot 10^{-21}$ & $2 \cdot 10^{-21}$ & $1 \cdot 10^{-21}$ & $8 \cdot 10^{-22}$ & $4 \cdot 10^{-22}$ \\
Presente trabalho & $(3.23)$ & $8 \cdot 10^{-47}$ & $5 \cdot 10^{-30}$ & $6 \cdot 10^{-25}$ & $6 \cdot 10^{-24}$ & $9 \cdot 10^{-24}$ & $8 \cdot 10^{-24}$ & $5 \cdot 10^{-24}$ & $3 \cdot 10^{-24}$ \\
\hline
\end{tabular}


Tabela 3.4 Erro relativo máximo de raízes da Eq. (1.6) após 2 iterações

\begin{tabular}{cccccccccc}
\hline \multirow{2}{*}{ Método } & \multirow{2}{*}{ Eq. } & \multicolumn{1}{c}{ Raiz } \\
\cline { 3 - 10 } a & & $1^{\underline{a}}$ & $4^{\underline{a}}$ & $8^{\underline{a}}$ & $16^{\underline{a}}$ & $32^{\underline{a}}$ & $64^{\underline{a}}$ & $128^{\underline{a}}$ \\
\hline Stevens e Luck simp. & $(2.6)$ & $1 \cdot 10^{-11}$ & $4 \cdot 10^{-8}$ & $7 \cdot 10^{-11}$ & $3 \cdot 10^{-12}$ & $2 \cdot 10^{-12}$ & $5 \cdot 10^{-13}$ & $1 \cdot 10^{-13}$ & $4 \cdot 10^{-14}$ \\
Stevens e Luck & $(2.3)$ & - & - & - & - & - & - & - & - \\
Newton-Raphson & $(3.6)$ & - & - & - & - & - & - & - & - \\
Chebyshev & $(3.14)$ & - & - & - & - & - & - & - & - \\
Halley & $(3.12)$ & $2 \cdot 10^{-23}$ & $6 \cdot 10^{-12}$ & $3 \cdot 10^{-13}$ & $6 \cdot 10^{-14}$ & $2 \cdot 10^{-14}$ & $6 \cdot 10^{-15}$ & $3 \cdot 10^{-15}$ & $2 \cdot 10^{-15}$ \\
Ostrowski & $(3.15)$ & - & - & - & - & - & - & - & - \\
Super-Halley & $(3.13)$ & - & - & - & - & - & - & - & - \\
Euler & $(3.7)$ & - & - & - & - & - & - & - & - \\
Traub (3a) & $(3.20)$ & - & - & - & - & - & - & - & - \\
Traub (6a) & $(3.19)$ & $2 \cdot 10^{-3}$ & $4 \cdot 10^{-3}$ & $9 \cdot 10^{-4}$ & $2 \cdot 10^{-4}$ & $5 \cdot 10^{-5}$ & $1 \cdot 10^{-5}$ & $3 \cdot 10^{-6}$ & $8 \cdot 10^{-7}$ \\
Traub (5a) & $(3.18)$ & - & - & - & - & - & - & - & - \\
Householder & $(3.17)$ & $2 \cdot 10^{-39}$ & $2 \cdot 10^{-22}$ & $3 \cdot 10^{-27}$ & $7 \cdot 10^{-31}$ & $6 \cdot 10^{-34}$ & $4 \cdot 10^{-33}$ & $2 \cdot 10^{-33}$ & $8 \cdot 10^{-34}$ \\
Traub (8a) & $(3.21)$ & $2 \cdot 10^{-8}$ & $7 \cdot 10^{-7}$ & $3 \cdot 10^{-8}$ & $2 \cdot 10^{-9}$ & $1 \cdot 10^{-10}$ & $7 \cdot 10^{-12}$ & $4 \cdot 10^{-13}$ & $3 \cdot 10^{-14}$ \\
Presente trabalho & $(3.23)$ & $1 \cdot 10^{-39}$ & $4 \cdot 10^{-19}$ & $2 \cdot 10^{-21}$ & $2 \cdot 10^{-22}$ & $5 \cdot 10^{-23}$ & $2 \cdot 10^{-23}$ & $7 \cdot 10^{-24}$ & $3 \cdot 10^{-24}$ \\
\hline
\end{tabular}

Tabela 3.5 Erro relativo máximo de raízes da Eq. (1.7) após 2 iterações

\begin{tabular}{cccccccccc}
\hline \multirow{2}{*}{ Método } & \multirow{2}{*}{ Eq. } & \multicolumn{7}{c}{ Raiz } \\
\cline { 3 - 9 } & & $1^{\underline{a}}$ & $2^{\underline{a}}$ & $4^{\underline{a}}$ & $8^{\underline{a}}$ & $16^{\underline{a}}$ & $32^{\underline{a}}$ & $64^{\underline{a}}$ & $128^{\underline{a}}$ \\
\hline Stevens e Luck simp. & $(2.6)$ & $1 \cdot 10^{-9}$ & $2 \cdot 10^{-7}$ & $1 \cdot 10^{-9}$ & $1 \cdot 10^{-11}$ & $6 \cdot 10^{-13}$ & $6 \cdot 10^{-13}$ & $2 \cdot 10^{-13}$ & $7 \cdot 10^{-14}$ \\
Stevens e Luck & $(2.3)$ & $2 \cdot 10^{-21}$ & $1 \cdot 10^{-11}$ & $5 \cdot 10^{-13}$ & $7 \cdot 10^{-14}$ & $2 \cdot 10^{-14}$ & $7 \cdot 10^{-15}$ & $3 \cdot 10^{-15}$ & $2 \cdot 10^{-15}$ \\
Newton-Raphson & $(3.6)$ & - & $6 \cdot 10^{-5}$ & $1 \cdot 10^{-4}$ & $8 \cdot 10^{-5}$ & $5 \cdot 10^{-5}$ & $3 \cdot 10^{-5}$ & $2 \cdot 10^{-5}$ & $8 \cdot 10^{-6}$ \\
Chebyshev & $(3.14)$ & - & $1 \cdot 10^{-7}$ & $9 \cdot 10^{-7}$ & $1 \cdot 10^{-6}$ & $1 \cdot 10^{-6}$ & $7 \cdot 10^{-7}$ & $4 \cdot 10^{-7}$ & $2 \cdot 10^{-7}$ \\
Halley & $(3.12)$ & $2 \cdot 10^{-22}$ & $3 \cdot 10^{-12}$ & $3 \cdot 10^{-13}$ & $6 \cdot 10^{-14}$ & $2 \cdot 10^{-14}$ & $7 \cdot 10^{-15}$ & $3 \cdot 10^{-15}$ & $2 \cdot 10^{-15}$ \\
Ostrowski & $(3.15)$ & $1 \cdot 10^{-4}$ & $2 \cdot 10^{-5}$ & $3 \cdot 10^{-5}$ & $2 \cdot 10^{-5}$ & $1 \cdot 10^{-5}$ & $8 \cdot 10^{-6}$ & $4 \cdot 10^{-6}$ & $2 \cdot 10^{-6}$ \\
Super-Halley & $(3.13)$ & - & $3 \cdot 10^{-8}$ & $2 \cdot 10^{-7}$ & $2 \cdot 10^{-7}$ & $2 \cdot 10^{-7}$ & $1 \cdot 10^{-7}$ & $6 \cdot 10^{-8}$ & $3 \cdot 10^{-8}$ \\
Euler & $(3.7)$ & - & $2 \cdot 10^{-8}$ & $9 \cdot 10^{-8}$ & $1 \cdot 10^{-7}$ & $9 \cdot 10^{-8}$ & $5 \cdot 10^{-8}$ & $3 \cdot 10^{-8}$ & $2 \cdot 10^{-8}$ \\
Traub (3a) & $(3.20)$ & - & $3 \cdot 10^{-11}$ & $2 \cdot 10^{-9}$ & $7 \cdot 10^{-9}$ & $9 \cdot 10^{-9}$ & $7 \cdot 10^{-9}$ & $4 \cdot 10^{-9}$ & $2 \cdot 10^{-9}$ \\
Traub (6a) & $(3.19)$ & $5 \cdot 10^{-4}$ & $1 \cdot 10^{-20}$ & $1 \cdot 10^{-21}$ & $6 \cdot 10^{-21}$ & $8 \cdot 10^{-21}$ & $7 \cdot 10^{-21}$ & $4 \cdot 10^{-21}$ & $3 \cdot 10^{-21}$ \\
Traub (5a) & $(3.18)$ & - & $3 \cdot 10^{-19}$ & $8 \cdot 10^{-21}$ & $6 \cdot 10^{-21}$ & $1 \cdot 10^{-20}$ & $8 \cdot 10^{-21}$ & $5 \cdot 10^{-21}$ & $3 \cdot 10^{-21}$ \\
Householder & $(3.17)$ & $4 \cdot 10^{-39}$ & $2 \cdot 10^{-23}$ & $2 \cdot 10^{-27}$ & $5 \cdot 10^{-31}$ & $1 \cdot 10^{-33}$ & $4 \cdot 10^{-33}$ & $3 \cdot 10^{-33}$ & $8 \cdot 10^{-34}$ \\
Traub (8a) & $(3.21)$ & $3 \cdot 10^{-31}$ & $2 \cdot 10^{-21}$ & $2 \cdot 10^{-22}$ & $1 \cdot 10^{-21}$ & $2 \cdot 10^{-21}$ & $1 \cdot 10^{-21}$ & $8 \cdot 10^{-22}$ & $4 \cdot 10^{-22}$ \\
Presente trabalho & $(3.23)$ & $2 \cdot 10^{-39}$ & $7 \cdot 10^{-20}$ & $2 \cdot 10^{-21}$ & $2 \cdot 10^{-22}$ & $5 \cdot 10^{-23}$ & $2 \cdot 10^{-23}$ & $7 \cdot 10^{-24}$ & $3 \cdot 10^{-24}$ \\
\hline
\end{tabular}

Através desses resultados observa-se que todos os métodos melhoram suas previsões a cada raiz, devido a elas terem comportamento mais suave. Entretanto, a necessidade de exatidão das raízes está justamente em ordem inversa. Estas raízes são utilizadas em séries que tem coeficientes decrescentes, e para uma determinada exatidão requerida para a soma da série, são necessárias exatidões maiores para as primeiras raízes.

As duas funções iterativas que convergiram em todos casos e levaram a resultados mais exatos foram os das Eqs. (3.17) e (3.23). Esta última será apresentada em detalhe devido a ela corresponder 
a uma equação quadrática e, portanto, ter seus termos agrupados como coeficientes, o que simplifica sua forma.

A utilização das funções iterativas mais elaboradas para os casos que envolvem funções elementares, em particular funções trigonométricas, torna-se mais atrativo devido às frequentes identidades que envolvem essas funções.

Portanto, os coeficientes da função iterativa da Eq. (3.23) são:

$$
\begin{gathered}
A=-\frac{1}{3}\left(f+c-3+\frac{x_{k}^{2}}{f+c+1}\right) \\
B=(c+1)\left(\frac{f+c}{2 x_{k}}+\frac{x_{k}}{6(f+c+1)}\right)+\frac{x_{k}}{3}
\end{gathered}
$$

para se obter as raízes da Eq. (1.5),

$$
\begin{gathered}
A=\left(-\left(4 x_{k}^{2}+1\right)(f+c)^{4}+16 x_{k}^{2}(f+c)^{3}-2 x_{k}^{2}\left(4 x_{k}^{2}+5\right)(f+c)^{2}+16 x_{k}^{4}(f+c)\right. \\
\left.-a^{4}\left(4 x_{k}^{2}-3\right)\right) /\left(6 x_{k}^{2} D\right) \\
B=\left(3(2 c+1)(f+c)^{4}+2\left(2 x_{k}^{2}-3 c-1\right)(f+c)^{3}+2\left((4 c+1) x_{k}^{2}+c\right)(f+c)^{2}\right. \\
\left.+2 x_{k}^{2}\left(2 x_{k}^{2}-c\right)(f+c)+x_{k}^{4}(2 c+3)\right) /\left(6 x_{k} D\right) \\
D=(f+c)^{2}(2(f+c)-1)+x_{k}^{2}(2(f+c)+1)
\end{gathered}
$$

para as raízes da Eq. (1.6), e

$$
\begin{gathered}
A=-\frac{1}{3}\left(f-c+4+\frac{x_{k}^{2}}{f-c}\right) \\
B=\frac{c}{2 x_{k}}(f-c+1)+\frac{x_{k}}{6}\left(\frac{c}{f-c}-2\right)
\end{gathered}
$$

para as raízes da Eq. (1.7).

Com estes coeficientes, e com os valores iniciais indicados no início da seção, pode-se obter a exatidão que se necessite com poucas iterações, conforme a Tabela 3.6, que inclui o erro da aproximação inicial (identificado por $k=0$ ).

Tabela 3.6 Erro relativo máximo da função iterativa da Eq. (3.23) com valores inicias dados pelas Eqs. (3.34) e (3.33) e coeficientes dados pelas Eqs. (3.38) a (3.44)

\begin{tabular}{cccc}
\hline$k$ & Eq. (1.5) & Eq. (1.6) & Eq. (1.7) \\
\hline 0 & $1 \cdot 10^{-2}$ & $3 \cdot 10^{-2}$ & $2 \cdot 10^{-2}$ \\
1 & $2 \cdot 10^{-7}$ & $1 \cdot 10^{-5}$ & $7 \cdot 10^{-6}$ \\
2 & $9 \cdot 10^{-24}$ & $4 \cdot 10^{-19}$ & $7 \cdot 10^{-20}$ \\
3 & $2 \cdot 10^{-87}$ & $9 \cdot 10^{-73}$ & $7 \cdot 10^{-76}$ \\
\hline
\end{tabular}


A primeira utilização desta equação ocorreu aqui, mesmo nesse trabalho que começou utilizando as funções já embutidas de sistemas algébricos computacionais para se calcular as raízes exatas que foram utilizadas para gerar os gráficos do capítulo da revisão bibliográfica, e que agora podem ser substituídas por esta, diminuindo significativamente o tempo computacional necessário. 


\section{AS TABELAS DE RAÍZES}

Apesar do avançado estágio atual de desenvolvimento de métodos numéricos para se obter raízes de equações, e de toda a acessibilidade a esses métodos, estando disponíveis até em smartphones e na internet, as tabelas de raízes que não tem expressões fechadas continuam sendo usadas, e os livros de condução de calor, tanto os elementares como os avançados, continuam a publicá-las. Isto ocorre, em parte, devido aos métodos numéricos necessitarem de enumeração e localização das raízes para serem aplicados e, no caso das presentes equações, haver uma dificuldade adicional devido à existência de infinitas raízes (FRANKEL, 1937, MIKHAILOV; ÖZIŞIK, 1986).

\subsection{As Tabelas com Passo Variável}

Hahn e Ozisik (2012) apresentam tabelas com as seis primeiras raízes das Eqs. (1.5), (1.6) e (1.7), com quatro algarismos decimais, onde a primeira coluna tem uma variável expressa em termos de $c$ que assume valores com passo não uniforme.

Essas tabelas são iguais às de Carslaw e Jaeger (1959) e são indicadas também por Arpaci (1966). Foram recalculadas as raízes utilizando os mesmos valores para a primeira coluna, obtendo a Tabela 4.1, a Tabela 4.2, e a Tabela 4.3, para as raízes das Eqs. (1.5), (1.6) e (1.7), respectivamente, e observou-se que os resultados publicados não apresentam erros (o que não ocorrerá com tabelas que serão apresentadas posteriormente).

Métodos como o da capacitância concentrada podem ser utilizados em vez de métodos de separação de variáveis quando o valor da variável $c$ que aparece nessas tabelas é baixo. Além disso, quando o valor de $c$ é alto, a condição de contorno de terceiro tipo pode ser aproximada pela de primeiro tipo, quando, então, o autovalor é dado por uma equação com solução fechada. Porém, é conveniente que as tabelas contenham amplos intervalos das variáveis independentes, incluindo os valores baixos e altos, para que o método geral possa ser aplicado também nos casos nos quais outros métodos mais simples existam. 
Tabela 4.1 Seis Primeiras Raízes da Eq. (1.5)

\begin{tabular}{|c|c|c|c|c|c|c|}
\hline$c$ & $\beta_{1}$ & $\beta_{2}$ & $\beta_{3}$ & $\beta_{4}$ & $\beta_{5}$ & $\beta_{6}$ \\
\hline 0 & 0 & 3,1416 & 6,2832 & 9,4248 & 12,5664 & 15,708 \\
\hline 0,001 & 0,0316 & 3,1419 & 6,2833 & 9,4249 & 12,5665 & 15,708 \\
\hline 0,002 & 0,0447 & 3,1422 & 6,2835 & 9,425 & 12,5665 & 15,7081 \\
\hline 0,004 & 0,0632 & 3,1429 & 6,2838 & 9,4252 & 12,5667 & 15,7082 \\
\hline 0,006 & 0,0774 & 3,1435 & 6,2841 & 9,4254 & 12,5668 & 15,7083 \\
\hline 0,008 & 0,0893 & 3,1441 & 6,2845 & 9,4256 & 12,567 & 15,7085 \\
\hline 0,01 & 0,0998 & 3,1448 & 6,2848 & 9,4258 & 12,5672 & 15,7086 \\
\hline 0,02 & 0,141 & 3,1479 & 6,2864 & 9,4269 & 12,568 & 15,7092 \\
\hline 0,04 & 0,1987 & 3,1543 & 6,2895 & 9,429 & 12,5696 & 15,7105 \\
\hline 0,06 & 0,2425 & 3,1606 & 6,2927 & 9,4311 & 12,5711 & 15,7118 \\
\hline 0,08 & 0,2791 & 3,1668 & 6,2959 & 9,4333 & 12,5727 & 15,7131 \\
\hline 0,1 & 0,3111 & 3,1731 & 6,2991 & 9,4354 & 12,5743 & 15,7143 \\
\hline 0,2 & 0,4328 & 3,2039 & 6,3148 & 9,4459 & 12,5823 & 15,7207 \\
\hline 0,3 & 0,5218 & 3,2341 & 6,3305 & 9,4565 & 12,5902 & 15,727 \\
\hline 0,4 & 0,5932 & 3,2636 & 6,3461 & 9,467 & 12,5981 & 15,7334 \\
\hline 0,5 & 0,6533 & 3,2923 & 6,3616 & 9,4775 & 12,606 & 15,7397 \\
\hline 0,6 & 0,7051 & 3,3204 & 6,377 & 9,4879 & 12,6139 & 15,746 \\
\hline 0,7 & 0,7506 & 3,3477 & 6,3923 & 9,4983 & 12,6218 & 15,7524 \\
\hline 0,8 & 0,791 & 3,3744 & 6,4074 & 9,5087 & 12,6296 & 15,7587 \\
\hline 0,9 & 0,8274 & 3,4003 & 6,4224 & 9,519 & 12,6375 & 15,765 \\
\hline 1 & 0,8603 & 3,4256 & 6,4373 & 9,5293 & 12,6453 & 15,7713 \\
\hline 1,5 & 0,9882 & 3,5422 & 6,5097 & 9,5801 & 12,6841 & 15,8026 \\
\hline 2 & 1,0769 & 3,6436 & 6,5783 & 9,6296 & 12,7223 & 15,8336 \\
\hline 3 & 1,1925 & 3,8088 & 6,704 & 9,724 & 12,7966 & 15,8945 \\
\hline 4 & 1,2646 & 3,9352 & 6,814 & 9,8119 & 12,8678 & 15,9536 \\
\hline 5 & 1,3138 & 4,0336 & 6,9096 & 9,8928 & 12,9352 & 16,0107 \\
\hline 6 & 1,3496 & 4,1116 & 6,9924 & 9,9667 & 12,9988 & 16,0654 \\
\hline 7 & 1,3766 & 4,1746 & 7,064 & 10,0339 & 13,0584 & 16,1177 \\
\hline 8 & 1,3978 & 4,2264 & 7,1263 & 10,0949 & 13,1141 & 16,1675 \\
\hline 9 & 1,4149 & 4,2694 & 7,1806 & 10,1502 & 13,166 & 16,2147 \\
\hline 10 & 1,4289 & 4,3058 & 7,2281 & 10,2003 & 13,2142 & 16,2594 \\
\hline 15 & 1,4729 & 4,4255 & 7,3959 & 10,3898 & 13,4078 & 16,4474 \\
\hline 20 & 1,4961 & 4,4915 & 7,4954 & 10,5117 & 13,542 & 16,5864 \\
\hline 30 & 1,5202 & 4,5615 & 7,6057 & 10,6543 & 13,7085 & 16,7691 \\
\hline 40 & 1,5325 & 4,5979 & 7,6647 & 10,7334 & 13,8048 & 16,8794 \\
\hline 50 & 1,54 & 4,6202 & 7,7012 & 10,7832 & 13,8666 & 16,9519 \\
\hline 60 & 1,5451 & 4,6353 & 7,7259 & 10,8172 & 13,9094 & 17,0026 \\
\hline 80 & 1,5514 & 4,6543 & 7,7573 & 10,8606 & 13,9644 & 17,0686 \\
\hline 100 & 1,5552 & 4,6658 & 7,7764 & 10,8871 & 13,9981 & 17,1093 \\
\hline$\infty$ & 1,5708 & 4,7124 & 7,854 & 10,9956 & 14,1372 & 17,2788 \\
\hline
\end{tabular}


Tabela 4.2 Seis Primeiras Raízes da Eq. (1.6)

\begin{tabular}{ccccccc}
\hline$c$ & $\beta_{1}$ & $\beta_{2}$ & $\beta_{3}$ & $\beta_{4}$ & $\beta_{5}$ & $\beta_{6}$ \\
\hline 0 & 0 & 3,8317 & 7,0156 & 10,1735 & 13,3237 & 16,4706 \\
0,01 & 0,1412 & 3,8343 & 7,017 & 10,1745 & 13,3244 & 16,4712 \\
0,02 & 0,1995 & 3,8369 & 7,0184 & 10,1754 & 13,3252 & 16,4718 \\
0,04 & 0,2814 & 3,8421 & 7,0213 & 10,1774 & 13,3267 & 16,4731 \\
0,06 & 0,3438 & 3,8473 & 7,0241 & 10,1794 & 13,3282 & 16,4743 \\
0,08 & 0,396 & 3,8525 & 7,027 & 10,1813 & 13,3297 & 16,4755 \\
0,1 & 0,4417 & 3,8577 & 7,0298 & 10,1833 & 13,3312 & 16,4767 \\
0,15 & 0,5376 & 3,8706 & 7,0369 & 10,1882 & 13,3349 & 16,4797 \\
0,2 & 0,617 & 3,8835 & 7,044 & 10,1931 & 13,3387 & 16,4828 \\
0,3 & 0,7465 & 3,9091 & 7,0582 & 10,2029 & 13,3462 & 16,4888 \\
0,4 & 0,8516 & 3,9344 & 7,0723 & 10,2127 & 13,3537 & 16,4949 \\
0,5 & 0,9408 & 3,9594 & 7,0864 & 10,2225 & 13,3611 & 16,5009 \\
0,6 & 1,0184 & 3,9841 & 7,1004 & 10,2322 & 13,3686 & 16,507 \\
0,7 & 1,0873 & 4,0085 & 7,1143 & 10,2419 & 13,3761 & 16,513 \\
0,8 & 1,149 & 4,0325 & 7,1282 & 10,2516 & 13,3835 & 16,5191 \\
0,9 & 1,2048 & 4,0562 & 7,1421 & 10,2613 & 13,391 & 16,5251 \\
1 & 1,2558 & 4,0795 & 7,1558 & 10,271 & 13,3984 & 16,5312 \\
1,5 & 1,4569 & 4,1902 & 7,2233 & 10,3188 & 13,4353 & 16,5612 \\
2 & 1,5994 & 4,291 & 7,2884 & 10,3658 & 13,4719 & 16,591 \\
3 & 1,7887 & 4,4634 & 7,4103 & 10,4566 & 13,5434 & 16,6499 \\
4 & 1,9081 & 4,6018 & 7,5201 & 10,5423 & 13,6125 & 16,7073 \\
5 & 1,9898 & 4,7131 & 7,6177 & 10,6223 & 13,6786 & 16,763 \\
6 & 2,049 & 4,8033 & 7,7039 & 10,6964 & 13,7414 & 16,8168 \\
7 & 2,0937 & 4,8772 & 7,7797 & 10,7646 & 13,8008 & 16,8684 \\
8 & 2,1286 & 4,9384 & 7,8464 & 10,8271 & 13,8566 & 16,9179 \\
9 & 2,1566 & 4,9897 & 7,9051 & 10,8842 & 13,909 & 16,965 \\
10 & 2,1795 & 5,0332 & 7,9569 & 10,9363 & 13,958 & 17,0099 \\
15 & 2,2509 & 5,1773 & 8,1422 & 11,1367 & 14,1576 & 17,2008 \\
20 & 2,288 & 5,2568 & 8,2534 & 11,2677 & 14,2983 & 17,3442 \\
30 & 2,3261 & 5,341 & 8,3771 & 11,4221 & 14,4748 & 17,5348 \\
40 & 2,3455 & 5,3846 & 8,4432 & 11,5081 & 14,5774 & 17,6508 \\
50 & 2,3572 & 5,4112 & 8,484 & 11,5621 & 14,6433 & 17,7272 \\
60 & 2,3651 & 5,4291 & 8,5116 & 11,599 & 14,6889 & 17,7807 \\
80 & 2,375 & 5,4516 & 8,5466 & 11,6461 & 14,7475 & 17,8502 \\
100 & 2,3809 & 5,4652 & 8,5678 & 11,6747 & 14,7834 & 17,8931 \\
$\infty$ & 2,4048 & 5,5201 & 8,6537 & 11,7915 & 14,9309 & 18,0711 \\
\hline & & & & & &
\end{tabular}


Tabela 4.3 Seis Primeiras Raízes da Eq. (1.7)

\begin{tabular}{|c|c|c|c|c|c|c|}
\hline$C$ & $\beta_{1}$ & $\beta_{2}$ & $\beta_{3}$ & $\beta_{1}$ & $\beta_{5}$ & $\beta_{6}$ \\
\hline $\begin{array}{c}0 \\
0,005\end{array}$ & $\begin{array}{c}0 \\
0,1224\end{array}$ & $\begin{array}{l}4.4934 \\
4,4945\end{array}$ & $\begin{array}{l}7.7253 \\
7.7259\end{array}$ & $\begin{array}{l}10.9041 \\
10,9046\end{array}$ & $\begin{array}{l}14.0662 \\
14,0665\end{array}$ & $\begin{array}{c}17.2208 \\
17.221\end{array}$ \\
\hline 0,01 & 0,173 & 4,4956 & 7,7265 & 10,905 & 14,0669 & 17,2213 \\
\hline 0,02 & 0,2445 & 4,4979 & 7,7278 & 10,906 & 14,0676 & 17,2219 \\
\hline 0,03 & 0,2991 & 4,5001 & 7,7291 & 10,9069 & 14,0683 & 17,2225 \\
\hline 0,04 & 0,345 & 4,5023 & 7,7304 & 10,9078 & 14,069 & 17,2231 \\
\hline 0,05 & 0,3854 & 4,5045 & 7,7317 & 10,9087 & 14,0697 & 17,2237 \\
\hline 0,06 & 0,4217 & 4,5068 & 7,733 & 10,9096 & 14,0705 & 17,2242 \\
\hline 0,07 & 0,4551 & 4,509 & 7,7343 & 10,9105 & 14,0712 & 17,2248 \\
\hline 0,08 & 0,486 & 4,5112 & 7,7356 & 10,9115 & 14,0719 & 17,2254 \\
\hline 0,09 & 0,515 & 4,5134 & 7,7369 & 10,9124 & 14,0726 & 17,226 \\
\hline 0,1 & 0,5423 & 4,5157 & 7,7382 & 10,9133 & 14,0733 & 17,2266 \\
\hline 0,15 & 0,6609 & 4,5268 & 7,7447 & 10,9179 & 14,0769 & 17,2295 \\
\hline 0,2 & 0,7593 & 4,5379 & 7,7511 & 10,9225 & 14,0804 & 17,2324 \\
\hline 0,3 & 0,9208 & 4,5601 & 7,7641 & 10,9316 & 14,0875 & 17,2382 \\
\hline 0,4 & 1,0528 & 4,5822 & 7,777 & 10,9408 & 14,0946 & 17,244 \\
\hline 0,5 & 1,1656 & 4,6042 & 7,7899 & 10,9499 & 14,1017 & 17,2498 \\
\hline 0,6 & 1,2644 & 4,6261 & 7,8028 & 10,9591 & 14,1088 & 17,2556 \\
\hline 0,7 & 1,3525 & 4,6479 & 7,8156 & 10,9682 & 14,1159 & 17,2614 \\
\hline 0,8 & 1,432 & 4,6696 & 7,8284 & 10,9774 & 14,123 & 17,2672 \\
\hline 0,9 & 1,5044 & 4,6911 & 7,8412 & 10,9865 & 14,1301 & 17,273 \\
\hline 1 & 1,5708 & 4,7124 & 7,854 & 10,9956 & 14,1372 & 17,2788 \\
\hline 1,1 & 1,632 & 4,7335 & 7,8667 & 11,0047 & 14,1442 & 17,2845 \\
\hline 1,2 & 1,6887 & 4,7544 & 7,8794 & 11,0137 & 14,1513 & 17,2903 \\
\hline 1,3 & 1,7414 & 4,7751 & 7,892 & 11,0228 & 14,1584 & 17,2961 \\
\hline 1,4 & 1,7906 & 4,7956 & 7,9045 & 11,0318 & 14,1654 & 17,3019 \\
\hline 1,5 & 1,8366 & 4,8158 & 7,9171 & 11,0408 & 14,1724 & 17,3076 \\
\hline 1,6 & 1,8798 & 4,8358 & 7,9295 & 11,0498 & 14,1795 & 17,3134 \\
\hline 1,7 & 1,9203 & 4,8556 & 7,9419 & 11,0588 & 14,1865 & 17,3192 \\
\hline 1,8 & 1,9586 & 4,875 & 7,9542 & 11,0677 & 14,1935 & 17,3249 \\
\hline 1,9 & 1,9947 & 4,8942 & 7,9665 & 11,0766 & 14,2005 & 17,3306 \\
\hline 2 & 2,0288 & 4,9132 & 7,9787 & 11,0855 & 14,2074 & 17,3364 \\
\hline 2,5 & 2,1746 & 5,0036 & 8,0385 & 11,1295 & 14,2421 & 17,3649 \\
\hline 3 & 2,2889 & 5,087 & 8,0962 & 11,1727 & 14,2764 & 17,3932 \\
\hline 4 & 2,4556 & 5,2329 & 8,2045 & 11,256 & 14,3434 & 17,449 \\
\hline 5 & 2,5704 & 5,354 & 8,3029 & 11,3348 & 14,408 & 17,5034 \\
\hline 6 & 2,6537 & 5,4544 & 8,3913 & 11,4086 & 14,4699 & 17,5562 \\
\hline 7 & 2,7165 & 5,5378 & 8,4703 & 11,4773 & 14,5288 & 17,6072 \\
\hline 8 & 2,7654 & 5,6078 & 8,5 & 11,5 & 14,5847 & 17,6562 \\
\hline 9 & 2,8044 & 5,6669 & 8,6031 & 11,5993 & 14,6 & 17,7032 \\
\hline 10 & 2,8363 & 5,7172 & 8,6587 & 11,6532 & 14,6869 & 17,7481 \\
\hline 11 & 2,8628 & 5,7606 & 8,7083 & 11,7027 & 14,7335 & 17,7908 \\
\hline 16 & 2,9476 & 5,908 & 8,8898 & 11,8959 & 14,9251 & 17,9742 \\
\hline 21 & 2,993 & 5,9921 & 9,0018 & 12,025 & 15,0625 & 18,1136 \\
\hline 31 & 3,0406 & 6,0831 & 9,1294 & 12,1807 & 15,238 & 18,3018 \\
\hline 41 & 3,0651 & 6,1311 & 9,1987 & 12,2688 & 15,3417 & 18,418 \\
\hline 51 & 3,0801 & 6,1606 & 9,242 & 12,3247 & 15,409 & 18,4953 \\
\hline 61 & 3,0901 & 6,1805 & 9,2715 & 12,3632 & 15,4558 & 18,5497 \\
\hline 81 & 3,1028 & 6,2058 & 9,3089 & 12,4124 & 15,5164 & 18,6209 \\
\hline 101 & 3,1105 & 6,2211 & 9,3317 & 12,4426 & 15,5537 & 18,665 \\
\hline$\infty$ & 3,1416 & 6,2832 & 9,4248 & 12,5664 & 15,708 & 18,8496 \\
\hline
\end{tabular}


O mais importante para se entender sobre o uso dessas tabelas é que os problemas que acompanham os livros para facilitar a absorção de seus conteúdos, podem ter enunciados com dados que levam a um valor idêntico aos que estão disponíveis na primeira coluna das tabelas, porém, os problemas reais, ao contrário, dificilmente terão dados assim. Então, o uso das tabelas presume utilização de interpolação e, consequentemente, a exatidão dos resultados obtidos pela utilização de tabelas é limitada pela exatidão dos valores que são obtidos por interpolação. Por exemplo, se for necessário saber o valor da primeira raiz $\beta_{1}$ da Eq. (1.5) para $c=0,14$, será necessário interpolar os valores de $c=0,1$ e $c=0,2$, que são 0,3111 e 0,4328 , para se obter $\beta_{1} \cong 0,35978$, enquanto que o valor exato é $\beta_{1}=0,36566$, o que representa um erro de $-0,016 \equiv-1,6 \%$.

O erro relativo dos valores de $\beta_{1}$ obtidos através de interpolação linear em função de $c$ é dado na Figura 4.1. Então, apesar dessas tabelas terem todos esses algarismos significativos, o que representa um erro de valores tabulados menor do que $0,06 \%$, para problemas práticos o uso dessa tabela pode levar a erro de até 1,6\%, que é maior do que o de expressões aproximadas que serão desenvolvidas.

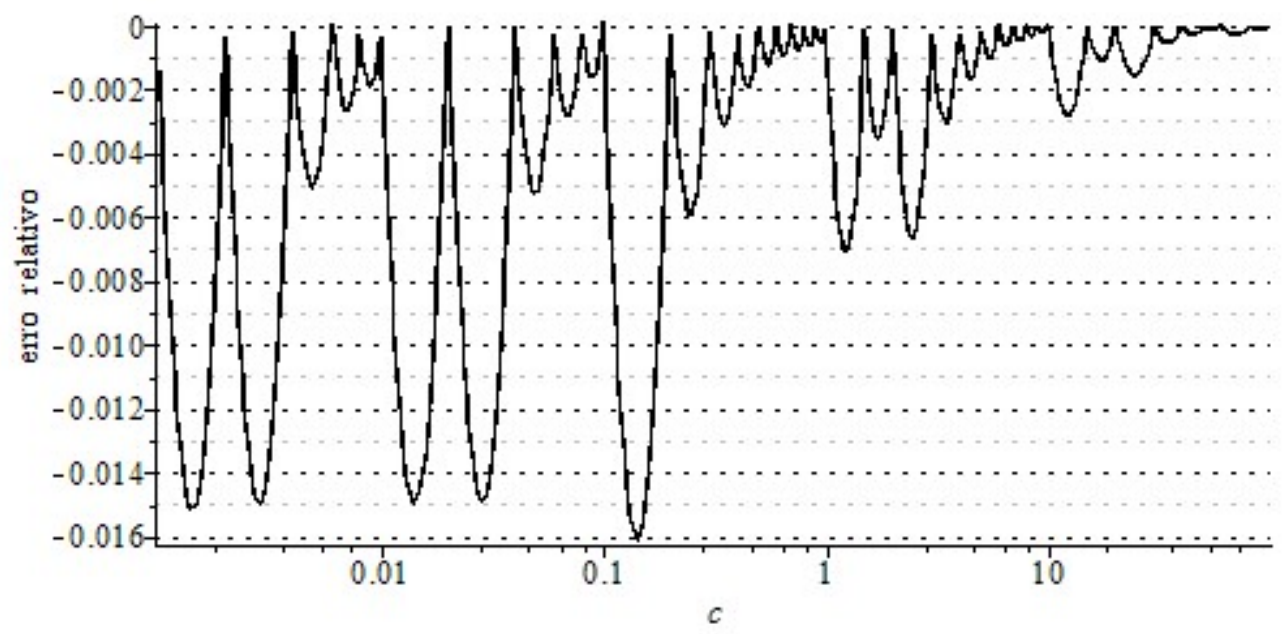

Figura 4.1 Erro relativo dos valores de $\beta_{1}$ obtidos através de interpolação linear da Tabela 4.1 para 0,001 $\leq c \leq 100$

Observe-se nesta figura que o erro é sempre negativo devido a curva dos valores exatos ter concavidade para baixo. Além disso, ele nunca é zero devido a limitação dos quatro algarismos decimais dos dados da tabela.

Devido a este gráfico ser semilogarítmico, $c$ não alcança o valor nulo. A interpolação linear da primeira raiz entre $0 \leq c \leq 0,001$ é:

$$
\beta_{1}(c) \cong 31,6 c
$$


que tem um erro relativo tendendo a $-100 \%$ quando $c$ tende a zero, como pode ser visto pela Eq. (2.12):

$$
\lim _{c \rightarrow 0^{+}}\left(\frac{31,6 c}{\sqrt{c}}\right)=0 \neq 1 \rightarrow \lim _{c \rightarrow 0^{+}}\left(\frac{31,6 c-\sqrt{c}}{\sqrt{c}}\right)=-1
$$

e com velocidade de aumento de seu valor absoluto que não é baixa, como pode ser visto pela Figura 4.2 .

Para $100<c<\infty$, a interpolação linear dessa e de qualquer outra raiz resultaria em funções constantes $\beta_{n} \cong n \pi$, as quais representam um erro de aproximadamente $1 \%$. Então, exclui-se os extremos de $c$ da avaliação dos erros de interpolação que será realizada.

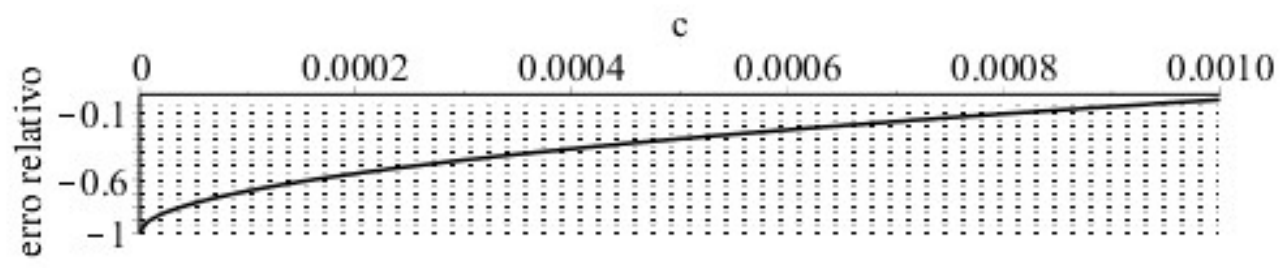

Figura 4.2 Erro relativo dos valores de $\beta_{1}$ obtidos através de interpolação linear da Tabela 4.1 para $0 \leq c \leq 0,001$

A Tabela 4.4 mostra o erro máximo de 1,6\% (em módulo) da primeira raiz da Eq. (1.5) obtida por interpolação linear, bem como o das demais raízes. Este erro pode ser diminuído com interpolações de maior ordem e essa tabela mostra que este erro, por exemplo, pode diminuir para $1,2 \%$ com interpolação de 7ํㅡㅁ ordem.

Os valores que não são mostrados nesta tabela, correspondem a erro maior do que o que é obtido com interpolações de menores ordens. Isto ocorre porque as curvas polinomiais podem oscilar muito quando o grau é alto, e o ajuste, então, é comprometido.

As Eqs. (1.6) e (1.7) levam a erro com valores muito próximos a estes apresentados para a Eq. (1.5). Então, o módulo do erro do resultado do cálculo da primeira raiz para estas três equações é de aproximadamente 1,5\% para interpolações lineares e de aproximadamente 1,2\% para interpolação de 7ํㅡ ordem (Tabela 4.5 e Tabela 4.6, para as Eqs. (1.6) e (1.7), respectivamente).

0 espaçamento menor entre os menores valores de $c$ permite uma exatidão mais uniforme, porém, este espaçamento irregular aumenta o número de operações computacionais necessárias para se obter interpolações de maior ordem. 
Tabela 4.4 Máximos módulos do erro relativo das raízes da Eq. (1.5) obtidas através de interpolação de várias ordens da Tabela 4.1 para $0,001 \leq c \leq 100$

\begin{tabular}{cccccccccc}
\hline Raiz & $1^{\underline{a}}$ & $2^{\underline{a}}$ & $3^{\underline{a}}$ & $4^{\underline{a}}$ & $5^{\underline{a}}$ & $6^{\underline{a}}$ & $7^{\underline{a}}$ & $8^{\underline{a}}$ & $9^{\underline{a}}$ \\
\hline$\beta_{1}$ & 0,016 & - & - & - & - & 0,013 & 0,012 & - & - \\
$\beta_{2}$ & 0,002 & 0,0008 & 0,0003 & - & 0,0002 & - & - & - & - \\
$\beta_{3}$ & 0,002 & 0,0007 & 0,0002 & - & 0,0001 & - & - & - & - \\
$\beta_{4}$ & 0,001 & 0,0005 & 0,0001 & 0,00008 & - & - & - & - & - \\
$\beta_{5}$ & 0,0009 & 0,0003 & 0,00009 & 0,00005 & - & - & - & - & - \\
$\beta_{6}$ & 0,0007 & 0,0002 & 0,00005 & - & - & - & - & - & - \\
\hline
\end{tabular}

Tabela 4.5 Máximos módulos do erro relativo das raízes da Eq. (1.6) obtidas através de interpolação de várias ordens da Tabela 4.2 para $0,01 \leq c \leq 100$

\begin{tabular}{cccccccccc}
\hline Raiz & $1^{\underline{\underline{a}}}$ & $2^{\underline{a}}$ & $3^{\underline{a}}$ & $4^{\underline{a}}$ & $5^{\underline{a}}$ & $6^{\underline{a}}$ & $7^{\underline{a}}$ & $8^{\underline{a}}$ & $9^{\underline{a}}$ \\
\hline$\beta_{1}$ & 0,015 & - & - & - & - & 0,013 & 0,012 & - & - \\
$\beta_{2}$ & 0,002 & 0,0009 & 0,0003 & - & 0,0002 & - & - & - & - \\
$\beta_{3}$ & 0,002 & 0,0007 & 0,0002 & - & 0,0001 & - & - & - & - \\
$\beta_{4}$ & 0,001 & 0,0005 & 0,0001 & 0,00006 & - & - & & - & - \\
$\beta_{5}$ & 0,0009 & 0,0003 & 0,00008 & 0,00005 & - & - & - & - & - \\
$\beta_{6}$ & 0,0007 & 0,0002 & 0,00004 & - & - & - & - & - & - \\
\hline
\end{tabular}

Tabela 4.6 Máximos módulos do erro relativo das raízes da Eq. (1.7) obtidas através de interpolação de várias ordens da Tabela 4.3, para $0,005 \leq c \leq 101$

\begin{tabular}{cccccccccc}
\hline Raiz & $1^{\underline{a}}$ & $2^{\underline{a}}$ & $3^{\underline{a}}$ & $4^{\underline{a}}$ & $5^{\underline{a}}$ & $6^{\underline{a}}$ & $7^{\underline{a}}$ & $8^{\underline{a}}$ & $9^{\underline{a}}$ \\
\hline$\beta_{1}$ & 0,015 & - & - & - & - & 0,013 & 0,011 & - & - \\
$\beta_{2}$ & 0,002 & 0,0008 & 0,0003 & - & 0,0001 & - & - & - & - \\
$\beta_{3}$ & 0,001 & 0,0006 & 0,0002 & 0,0001 & - & - & - & - & - \\
$\beta_{4}$ & 0,001 & 0,0004 & 0,0001 & 0,00005 & - & - & & - & - \\
$\beta_{5}$ & 0,0008 & 0,0003 & 0,00007 & 0,00005 & - & - & - & - & - \\
$\beta_{6}$ & 0,0007 & 0,0002 & 0,00004 & - & - & - & - & - & - \\
\hline
\end{tabular}

Devido a este aumento do número de operações e diminuição muito pequena do erro com interpolações de maior ordem, devido a não ser informado nestas tabelas qual a máxima ordem que poderia ser usada, e devido a importância do erro do primeiro autovalor, pode-se associar a estas tabelas a um erro relativo máximo da ordem de 1,5\%. 


\subsection{As Tabelas com Passo Constante}

Zucker (1964) apresenta tabelas com as nove primeiras raízes das Eqs. (1.5) e (1.7) com cinco algarismos decimais, e das cinco primeiras raízes da Eq. (1.6) com quatro algarismos decimais. Pela divisão das tabelas em duas partes, com uma engenhosa escolha da variável da primeira coluna, podese obter todos os valores não negativos de $c$, através de uma variável da primeira coluna com passo constante, o que facilita interpolações de maiores ordens.

Utilizando as mesmas formas, obtivemos a Tabela 4.7, a Tabela 4.8 e a Tabela 4.9, e observamos alguns erros de arredondamento na tabela publicada equivalente à Tabela 4.7, como por exemplo em $c=0,05$, onde o valor de $\beta_{6}$ apresentado é 15,71114 enquanto que o valor correto é $15,7111457 \ldots$, e em $c=1 / 0,9$, onde o valor de $\beta_{8}$ apresentado é 22,04151 enquanto que o valor correto é 22,04151586 ... (ambos deveriam ter sido arredondados para cima). Contudo, estes erros estão dentro da tolerância assegurada por este manual que é de $6 / 10$ do último algarismo significativo.

No rodapé da tabela publicada indica-se que o erro de máximo módulo das raízes da Eq. (1.5) obtidas através de interpolação linear da Tabela 4.7 para $0 \leq c<1$ seria de $7,0 \cdot 10^{-4}$. Recalculamos todos erros (Tabela 4.10) e verificamos que o máximo erro é, na verdade, de $8,0 \cdot 10^{-4}$ para $\beta_{1}$. De fato, para $c=0,22436$ o valor de $\beta_{1}$ é 0,45666 ... enquanto que a interpolação linear leva a $0,45586 \ldots$ e, então, $0,45586-0,45666=-0,00080$.

A tabela publicada equivalente à Tabela 4.8 foi parcialmente extraída de Carslaw e Jaeger (1959) e não tem erros de arredondamento. Já a equivalente a Tabela 4.9 tem alguns erros dentro da tolerância, e um erro fora da tolerância porque mostra $\beta_{6}=17,27875$ para $c=0,95$, enquanto que o valor correto é 17,2787596 (este valor poderia ser obtido a partir do valor correto que está numa raiz equivalente na Tabela 4.7).

Os erros máximos para o intervalo maior não foram indicados no rodapé da tabela publicada, mas também está apresentado na Tabela 4.10. Por fim, também não foram indicados os erros de máximos módulos obtidos através de interpolação linear da Tabela 4.8 e da Tabela 4.9 e em vez deles, será preferível se apresentar o erro relativo.

Os máximos módulos do erro relativo das raízes obtidas a partir da Tabela 4.7, da Tabela 4.8 e da Tabela 4.9 estão respectivamente indicados na Tabela 4.11, Tabela 4.12 e Tabela 4.13. Não foram excluídos delas os valores que são maiores do que os que são obtidos com interpolações de menor ordem, porque, apesar das interpolações de maior ordem serem mais recomendáveis com tabelas de passos constantes, deve-se atentar ao fato de que o erro pode aumentar em parte dos casos. 
Tabela 4.7 Nove Primeiras Raízes da Eq. (1.5)

\begin{tabular}{|c|c|c|c|c|c|c|c|c|c|}
\hline$C$ & $\beta_{1}$ & $\beta_{2}$ & $\beta_{3}$ & $\beta_{4}$ & $\beta_{5}$ & $\beta_{6}$ & $\beta_{7}$ & $\beta_{8}$ & $\beta_{9}$ \\
\hline 0 & 0 & 3,14159 & 6,28319 & 9,42478 & 56637 & ,70796 & 18,84956 & 21,99115 & 5121 \\
\hline 0,05 & & & & & & & & & \\
\hline 0,1 & & 1731 & 6,29906 & & & & & & \\
\hline 0 & 7788 & 1886 & & & & & & & \\
\hline & & & & & & & & & \\
\hline 0,25 & 48009 & 3,2191 & 6,32 & & 2,58623 & 2386 & 18,86281 & & \\
\hline 0,3 & 52179 & 3,23 & & & & & & & \\
\hline & & & & & & & & & \\
\hline 0,4 & 59324 & 26355 & 6,3 & 467 & & 15,7 & 18,87075 & & \\
\hline 0,45 & & 2 & 6,3 & & & & & & \\
\hline & & & & & & & & & \\
\hline 0,55 & & 43 & 6,3 & 9,4 & & 15 & & & \\
\hline & & 7 & & & & & & & \\
\hline 0,65 & & 33413 & & & & 15, & & & \\
\hline & & & & & & 15,7 & 18, & & \\
\hline 75 & & & & & & & & & \\
\hline & & 3,37438 & & & & 15,7 & & & \\
\hline 0,85 & & 44 & & & & & & & \\
\hline & & & & & & & & & \\
\hline 0,95 & & 3,41 & & & & & & & \\
\hline 1 & & 3,42562 & & & & 15,77128 & 18,90241 & & \\
\hline $1 / c$ & $\beta_{1}$ & $\beta_{2}$ & $\beta_{3}$ & $\beta_{4}$ & $\beta_{5}$ & $\beta_{6}$ & $\beta_{7}$ & & \\
\hline 1 & & & & & & & 00021 & & \\
\hline 0,95 & & & & & & & & & \\
\hline & & & & & & 15,77 & 18,90825 & & \\
\hline 0,85 & & 59 & & & & $15,7 \varepsilon$ & 18,91168 & & \\
\hline & & & & & & & & & \\
\hline 0 , & & & & & & 15,7 & 18,9 & & \\
\hline & & & & & & 15,7 & 249 & & \\
\hline 0,65 & & & & & & & 18,93065 & & \\
\hline & & & & & & 15,8 & 18,93734 & & \\
\hline 0,55 & & & & & & & & & \\
\hline & & 3,6436 & & & & 15,8 & 18,95468 & 22 & \\
\hline 0,45 & & 3,68433 & & & 907 & 15,84728 & 18,96619 & 14 & \\
\hline & & & & & & 15,86426 & 18,98052 & 377 & \\
\hline 0,35 & & 3,78784 & 6,6 & & & 15,88591 & 18,99882 & & \\
\hline & & & & & & 15,91443 & 19,02302 & & \\
\hline 0,25 & & 3,93516 & 6,8 & 188 & 12,86776 & 15,95363 & 19,05645 & 22 & 25 \\
\hline & & & & & & 16,0 & 19,10552 & & \\
\hline 0,15 & & 4,15504 & & & & 16,10 & 19,18401 & & \\
\hline & & 4,3058 & 7,2 & 10,200 & 13,21419 & 16,25936 & 19,32703 & 22 & 25 , \\
\hline & & & & & & 16,58639 & & & \\
\hline 0 & 1,57080 & 4,71239 & 7,85398 & 10,99557 & 14,13717 & 17,27876 & 20,42035 & 23,56194 & 26,70354 \\
\hline
\end{tabular}


Tabela 4.8 Cinco Primeiras Raízes da Eq. (1.6)

\begin{tabular}{cccccc}
\hline$c$ & $\beta_{1}$ & $\beta_{2}$ & $\beta_{3}$ & $\beta_{4}$ & $\beta_{5}$ \\
\hline 0 & 0 & 3,8317 & 7,0156 & 10,1735 & 13,3237 \\
0,02 & 0,1995 & 3,8369 & 7,0184 & 10,1754 & 13,3252 \\
0,04 & 0,2814 & 3,8421 & 7,0213 & 10,1774 & 13,3267 \\
0,06 & 0,3438 & 3,8473 & 7,0241 & 10,1794 & 13,3282 \\
0,08 & 0,396 & 3,8525 & 7,027 & 10,1813 & 13,3297 \\
0,1 & 0,4417 & 3,8577 & 7,0298 & 10,1833 & 13,3312 \\
0,2 & 0,617 & 3,8835 & 7,044 & 10,1931 & 13,3387 \\
0,4 & 0,8516 & 3,9344 & 7,0723 & 10,2127 & 13,3537 \\
0,6 & 1,0184 & 3,9841 & 7,1004 & 10,2322 & 13,3686 \\
0,8 & 1,149 & 4,0325 & 7,1282 & 10,2516 & 13,3835 \\
1 & 1,2558 & 4,0795 & 7,1558 & 10,271 & 13,3984 \\
\hline $1 / c$ & $\beta_{1}$ & $\beta_{2}$ & $\beta_{3}$ & $\beta_{4}$ & $\beta_{5}$ \\
\hline 1 & 1,2558 & 4,0795 & 7,1558 & 10,271 & 13,3984 \\
0,8 & 1,3659 & 4,1361 & 7,1898 & 10,295 & 13,4169 \\
0,6 & 1,5095 & 4,2249 & 7,2453 & 10,3346 & 13,4476 \\
0,4 & 1,706 & 4,3818 & 7,3508 & 10,4118 & 13,5079 \\
0,2 & 1,9898 & 4,7131 & 7,6177 & 10,6223 & 13,6786 \\
0,1 & 2,1795 & 5,0332 & 7,9569 & 10,9363 & 13,958 \\
0,08 & 2,2218 & 5,1172 & 8,0624 & 11,0477 & 14,0666 \\
0,06 & 2,2656 & 5,2085 & 8,1852 & 11,1864 & 14,21 \\
0,04 & 2,3108 & 5,3068 & 8,3262 & 11,3575 & 14,3996 \\
0,02 & 2,3572 & 5,4112 & 8,484 & 11,5621 & 14,6433 \\
0 & 2,4048 & 5,5201 & 8,6537 & 11,7915 & 14,9309 \\
\hline
\end{tabular}

Tabela 4.9 Nove Primeiras Raízes da Eq. (1.7)

\begin{tabular}{cccccccccc}
\hline (c-1)-1 & $\beta_{1}$ & $\beta_{2}$ & $\beta_{3}$ & $\beta_{4}$ & $\beta_{5}$ & $\beta_{6}$ & $\beta_{7}$ & $\beta_{8}$ & $\beta_{9}$ \\
\hline 1 & 2,02876 & 4,91318 & 7,97867 & 11,08554 & 14,20744 & 17,33638 & 20,46917 & 23,60428 & 26,74092 \\
0,95 & 2,04597 & 4,92303 & 7,98505 & 11,09021 & 14,2111 & 17,33939 & 20,47173 & 23,60651 & 26,74288 \\
0,9 & 2,06453 & 4,93389 & 7,99212 & 11,09538 & 14,21517 & 17,34274 & 20,47457 & 23,60897 & 26,74506 \\
0,85 & 2,0846 & 4,94592 & 7,99999 & 11,10116 & 14,21971 & 17,34648 & 20,47774 & 23,61173 & 26,74749 \\
0,8 & 2,10638 & 4,9593 & 8,00881 & 11,10764 & 14,22482 & 17,35068 & 20,48131 & 23,61483 & 26,75023 \\
0,75 & 2,13008 & 4,97428 & 8,01875 & 11,11496 & 14,23059 & 17,35543 & 20,48535 & 23,61834 & 26,75333 \\
0,7 & 2,15598 & 4,99116 & 8,03004 & 11,12331 & 14,23717 & 17,36086 & 20,48996 & 23,62235 & 26,75688 \\
0,65 & 2,1844 & 5,01031 & 8,04298 & 11,1329 & 14,24475 & 17,36711 & 20,49528 & 23,62697 & 26,76096 \\
0,6 & 2,21571 & 5,03222 & 8,05794 & 11,14403 & 14,25357 & 17,37439 & 20,50147 & 23,63235 & 26,76573 \\
0,55 & 2,25037 & 5,0575 & 8,07544 & 11,15712 & 14,26395 & 17,38298 & 20,50877 & 23,63871 & 26,77135 \\
0,5 & 2,28893 & 5,08699 & 8,09616 & 11,17271 & 14,27635 & 17,39324 & 20,51752 & 23,64632 & 26,77809 \\
0,45 & 2,33208 & 5,12176 & 8,12108 & 11,19159 & 14,29143 & 17,40574 & 20,52818 & 23,65561 & 26,78631 \\
0,4 & 2,38064 & 5,16331 & 8,15156 & 11,21491 & 14,31012 & 17,42129 & 20,54146 & 23,66719 & 26,79656 \\
0,35 & 2,43566 & 5,2137 & 8,18965 & 11,2444 & 14,33392 & 17,44113 & 20,55844 & 23,68201 & 26,80971 \\
0,3 & 2,4984 & 5,27587 & 8,23845 & 11,28284 & 14,36517 & 17,46732 & 20,58092 & 23,70167 & 26,82716 \\
0,25 & 2,57043 & 5,35403 & 8,30293 & 11,33483 & 14,40797 & 17,50343 & 20,61203 & 23,72895 & 26,85142 \\
\hline
\end{tabular}




\begin{tabular}{|c|c|c|c|c|c|c|c|c|c|}
\hline 0,2 & & 45435, & 39135 & 11,40863 & 4,46987 & 17,55621 & 20,65782 & 23,76928 & 26,8874 \\
\hline 0,15 & & & & 11,52018 & & 17,64009 & 20,73148 & & \\
\hline 0,1 & 86277 & & & & & & & & \\
\hline 0,05 & 99304 & 5,99209 & ,00185 & 12,02503 & 5,06247 & & & & \\
\hline 0 & 14159 & 6,28319 & ,42478 & 12,56637 & 15,70796 & 8,84956 & 21,99115 & & 28,27433 \\
\hline$c$ & $\beta_{1}$ & $\beta_{2}$ & $\beta_{3}$ & $\beta_{4}$ & $\beta_{5}$ & $\beta_{6}$ & $\beta_{7}$ & $\beta_{8}$ & $\beta_{9}$ \\
\hline 0 & 0 & & 72525 & 10,90412 & 14,06619 & 7,22076 & & 23,51945 & \\
\hline 0,05 & ,38537 & 4,50454 & ,73172 & 10,90871 & 14,06975 & 17,22366 & 20,37 & 158 & 26,66793 \\
\hline 0,1 & & 4,51566 & 7,7382 & 10,91 & & & 521 & & \\
\hline 0,15 & 66086 & 4,52678 & 7,74467 & 10,91 & 14,07 & & & & \\
\hline 0,2 & 0,75931 & 4,53789 & 7,75114 & 10,92 & $14,0 \varepsilon$ & & & & 355 \\
\hline 0,25 & & & & 10,9 & $14, c$ & & & & \\
\hline 0,3 & 0,92079 & 4,56 & 7,76 & 10,9 & 14,0 & & & & \\
\hline 0,35 & 0,98966 & 4,57 & 7,77053 & 10,9 & 14,0 & & & & 918 \\
\hline 0,4 & & & & $10, \subseteq$ & 14,0 & & & & \\
\hline 0,45 & & 4,59321 & 44 & 10, & 14,0 & & & & \\
\hline 0,5 & 56 & 4,60 & 7,7 & 10,9 & 14,1 & & & & \\
\hline 0,55 & & 4,61 & & 10,9 & 14,1 & & & & \\
\hline 0,6 & & 4,62 & & 10, & & & & & \\
\hline 0,65 & 65 & 4,63 & 9 & $10, \subseteq$ & 14 & & & & 42 \\
\hline 0,7 & & 4,64 & & 10, & 14, & & & & \\
\hline 0,75 & & 4,65 & & 10, & & & & & \\
\hline 0,8 & 1,4 & 4,66 & & 10 & 14, & & & & \\
\hline 0,85 & & $4,6 \varepsilon$ & & 10, & & & & & \\
\hline 0,9 & 1,50442 & 4,69 & 7,84 & & & & & & \\
\hline 0,95 & & & & & & & & & \\
\hline 1 & & 4,71 & & 10, & & & & & \\
\hline 1,05 & & 4,72 & 7,8 & $11,($ & & & & & \\
\hline 1,1 & & 4,73 & & 11, & 14 & & & & \\
\hline 1,15 & & & & & & & & & \\
\hline 1,2 & 3868 & 4,75 & & 11, & & & & & 26 , \\
\hline 1,25 & 51 & $4,7 €$ & & 11, & 14, & & & & \\
\hline 1,3 & & 4,77 & & & & & & & \\
\hline 1,35 & & & & & 14, & & & & \\
\hline 1,4 & 58 & 4,79 & 7,904 & 11,0 & & & & & \\
\hline 1,45 & 1,8 & 4,80 & & 11, & 14, & & & & \\
\hline 1,5 & & 4,81 & & & 14 & & & & \\
\hline 1,55 & & 4,82587 & 7,92329 & 11,0 & 14,1 & & & & 26 \\
\hline 1,6 & 1,87976 & 4,83583 & & 11,0 & 14,1 & & 68 & 38 & 26, \\
\hline 1,65 & & 4,84 & & 11, & 14,1 & & & & 26,7 \\
\hline 1,7 & 1,92035 & 4,85557 & 7,94189 & 11,0 & 14,18647 & & 20,45456 & 161 & 26,72972 \\
\hline 1,75 & 1,93974 & 4,86534 & & 11,0 & 14 & & & & 26 , \\
\hline 1,8 & & 4,87504 & & 11,06773 & 14,19347 & & & & \\
\hline 1,85 & 1,97687 & 4,88468 & 7,96036 & 11,07219 & 14,19697 & 17,32777 & 20,46187 & 23,59795 & 26,73532 \\
\hline 1,9 & & 4,89425 & 7,96648 & 11,07665 & 14,20046 & & & & 26,73719 \\
\hline 1,95 & 2,01194 & 4,90375 & 7,97258 & & 14,20395 & 17,33351 & 20,46674 & 23,60217 & 26,73905 \\
\hline 2 & 2,02876 & 4,91318 & 7,97867 & 11,08554 & 14,20744 & 17,33638 & 20,46917 & 23,60428 & 26,74092 \\
\hline
\end{tabular}


Tabela 4.10 Máximos módulos do erro relativo das raízes obtidas através de interpolação linear da Tabela 4.7

\begin{tabular}{ccc}
\hline Raiz & $0 \leq c<1$ & $1 \leq \mathrm{c}<\infty$ \\
\hline$\beta_{1}$ & $8,0 \cdot 10^{-4}$ & $9,6 \cdot 10^{-4}$ \\
$\beta_{2}$ & $2,5 \cdot 10^{-5}$ & $4,6 \cdot 10^{-3}$ \\
$\beta_{3}$ & $6,5 \cdot 10^{-6}$ & $1,2 \cdot 10^{-2}$ \\
$\beta_{4}$ & $6,4 \cdot 10^{-6}$ & $2,0 \cdot 10^{-2}$ \\
$\beta_{5}$ & $5,5 \cdot 10^{-6}$ & $3,5 \cdot 10^{-2}$ \\
$\beta_{6}$ & $5,7 \cdot 10^{-6}$ & $5,3 \cdot 10^{-2}$ \\
$\beta_{7}$ & $4,5 \cdot 10^{-6}$ & $7,5 \cdot 10^{-2}$ \\
$\beta_{8}$ & $5,6 \cdot 10^{-6}$ & $9,8 \cdot 10^{-2}$ \\
$\beta_{9}$ & $5,4 \cdot 10^{-6}$ & $1,2 \cdot 10^{-1}$ \\
\hline
\end{tabular}

Os erros da Tabela 4.11 parecerem menores porque a situação crítica foi excluída, que é a do cálculo da primeira raiz com o valor da variável $c$ baixo. Então, para esta raiz não foi considerado o intervalo $c<0,2$ e por isso foi encontrado um erro de apenas $0,2 \%=2 \cdot 10^{-3}$. 0 autor recomenda que neste caso, se utilize:

$$
\beta_{1}=\sqrt{c}\left(1-\frac{1}{6} c+\frac{11}{360} c^{2}-\frac{1}{432} c^{3}+\cdots\right)
$$

o que reduz a utilidade da tabela, já que esta raiz é a mais importante, e estes valores da variável $c$ não são tão baixos.

Então, devido a primeira raiz ser a mais importante, pode-se dizer que o erro relativo máximo destas tabelas de passo constante é da ordem de $2 \%$ quando se utiliza interpolação linear, e de 0,5\% se forem utilizadas interpolações de até $8^{a}$ ordem.

Não serão discutidas em detalhe como as interpolações de maior ordem foram efetuadas para pontos dos extremos dos intervalos, nem em que regiões foram encontrados os maiores valores do erro, porque não se tem como objetivo um maior aprofundamento na análise da utilização destas tabelas, mas apenas informar as ordens de grandeza dos valores do erro que são obtidos com sua utilização para efeito de comparação com os que serão obtidos com as expressões aproximadas. 
Tabela 4.11 Máximos módulos do erro relativo das raízes obtidas através de interpolação da Tabela 4.7, considerando todo o intervalo c $>0$ (ou c $\geq 0,2$ no caso de interpolação de $1^{\text {ă }}$ ordem de $\beta_{1}$ )

\begin{tabular}{|c|c|c|c|c|c|c|c|c|c|}
\hline \multirow{2}{*}{ Raiz } & \multicolumn{9}{|c|}{ Ordem da Interpolação } \\
\hline & $1^{\underline{a}}$ & $2^{\underline{a}}$ & $3^{\underline{a}}$ & $4 \underline{a}$ & $5^{\underline{a}}$ & $6^{\mathrm{a}}$ & $7 \underline{a}$ & $8^{\underline{a}}$ & $9 \underline{\underline{a}}$ \\
\hline$\beta_{1}$ & $2 \cdot 10^{-3}$ & $3 \cdot 10^{-4}$ & $5 \cdot 10^{-5}$ & $5 \cdot 10^{-5}$ & $2 \cdot 10^{-5}$ & $2 \cdot 10^{-5}$ & $9 \cdot 10^{-6}$ & $2 \cdot 10^{-4}$ & $1 \cdot 10^{-4}$ \\
\hline$\beta_{2}$ & $1 \cdot 10^{-3}$ & $1 \cdot 10^{-4}$ & $1 \cdot 10^{-4}$ & $5 \cdot 10^{-5}$ & $2 \cdot 10^{-5}$ & $7 \cdot 10^{-6}$ & $6 \cdot 10^{-6}$ & $1 \cdot 10^{-5}$ & $2 \cdot 10^{-5}$ \\
\hline$\beta_{3}$ & $2 \cdot 10^{-3}$ & $2 \cdot 10^{-4}$ & $1 \cdot 10$ & $2 \cdot 10^{-4}$ & $1 \cdot 10^{-4}$ & $8 \cdot 10$ & $4 \cdot 10^{-5}$ & $1 \cdot 1$ & $9 \cdot 10^{-6}$ \\
\hline$\beta_{4}$ & $2 \cdot 10^{-3}$ & $4 \cdot 10^{-4}$ & $5 \cdot 10^{-4}$ & $5 \cdot 10^{-4}$ & $4 \cdot 10^{-4}$ & $3 \cdot 10^{-4}$ & $2 \cdot 10^{-4}$ & $1 \cdot 10^{-4}$ & $9 \cdot 10^{-5}$ \\
\hline$\beta_{5}$ & $3 \cdot 10^{-3}$ & $6 \cdot 10^{-4}$ & $5 \cdot 10^{-4}$ & $6 \cdot 10^{-4}$ & $6 \cdot 10^{-4}$ & $5 \cdot 10^{-4}$ & $5 \cdot 10^{-4}$ & $4 \cdot 10^{-4}$ & $3 \cdot 10^{-4}$ \\
\hline$\beta_{6}$ & $3 \cdot 10^{-3}$ & $8 \cdot 10^{-4}$ & $5 \cdot 10^{-4}$ & $7 \cdot 10^{-4}$ & $7 \cdot 10^{-4}$ & $7 \cdot 10^{-4}$ & $7 \cdot 10^{-4}$ & $6 \cdot 10^{-4}$ & $6 \cdot 10^{-4}$ \\
\hline$\beta_{7}$ & $4 \cdot 10^{-3}$ & $1 \cdot 10^{-3}$ & $3 \cdot 10^{-4}$ & $6 \cdot 10^{-4}$ & $8 \cdot 10^{-4}$ & $8 \cdot 10^{-4}$ & $8 \cdot 10^{-4}$ & $8 \cdot 10^{-4}$ & $8 \cdot 10^{-4}$ \\
\hline$\beta_{8}$ & $4 \cdot 10^{-3}$ & $1 \cdot 10^{-3}$ & $4 \cdot 10^{-4}$ & $5 \cdot 10^{-4}$ & $7 \cdot 10^{-4}$ & $8 \cdot 10^{-4}$ & $8 \cdot 10^{-4}$ & $9 \cdot 10^{-4}$ & $9 \cdot 10^{-4}$ \\
\hline$\beta_{9}$ & $5 \cdot 10^{-3}$ & $2 \cdot 10^{-3}$ & $6 \cdot 10^{-4}$ & $3 \cdot 10^{-4}$ & $5 \cdot 10^{-4}$ & $7 \cdot 10^{-4}$ & $8 \cdot 10^{-4}$ & $8 \cdot 10^{-4}$ & $9 \cdot 10^{-4}$ \\
\hline
\end{tabular}

Tabela 4.12 Máximos módulos do erro relativo das raízes obtidas através de interpolação da Tabela 4.8, considerando todo o intervalo $c>0$ (ou $c \geq 0,02$ no caso de interpolação de $1^{a}$ ordem de $\beta_{1}$ )

\begin{tabular}{cccccccccc}
\hline \multirow{2}{*}{ Raiz } & \multicolumn{8}{c}{ Ordem da Interpolação } \\
\cline { 2 - 10 } & $1^{1^{\mathrm{a}}}$ & $2^{\mathrm{a}}$ & $3^{\mathrm{a}}$ & $4^{\mathrm{a}}$ & $5^{\mathrm{a}}$ & $6^{\mathrm{a}}$ & $7^{\mathrm{a}}$ & $8^{\mathrm{a}}$ & $9^{\mathrm{a}}$ \\
\hline$\beta_{1}$ & $2 \cdot 10^{-2}$ & $5 \cdot 10^{-2}$ & $2 \cdot 10^{-2}$ & $1 \cdot 10^{-2}$ & $9 \cdot 10^{-3}$ & $8 \cdot 10^{-3}$ & $5 \cdot 10^{-2}$ & $8 \cdot 10^{-1}$ & $1 \cdot 10^{-1}$ \\
$\beta_{2}$ & $8 \cdot 10^{-3}$ & $4 \cdot 10^{-3}$ & $4 \cdot 10^{-3}$ & $5 \cdot 10^{-4}$ & $7 \cdot 10^{-4}$ & $8 \cdot 10^{-4}$ & $1 \cdot 10^{-2}$ & $2 \cdot 10^{-2}$ & $7 \cdot 10^{-2}$ \\
$\beta_{3}$ & $5 \cdot 10^{-3}$ & $4 \cdot 10^{-3}$ & $2 \cdot 10^{-3}$ & $1 \cdot 10^{-3}$ & $1 \cdot 10^{-3}$ & $2 \cdot 10^{-3}$ & $8 \cdot 10^{-3}$ & $1 \cdot 10^{-1}$ & $6 \cdot 10^{-1}$ \\
$\beta_{4}$ & $3 \cdot 10^{-3}$ & $4 \cdot 10^{-3}$ & $2 \cdot 10^{-3}$ & $2 \cdot 10^{-3}$ & $1 \cdot 10^{-3}$ & $4 \cdot 10^{-3}$ & $6 \cdot 10^{-3}$ & $1 \cdot 10^{-1}$ & $2 \cdot 10^{0}$ \\
$\beta_{5}$ & $3 \cdot 10^{-3}$ & $3 \cdot 10^{-3}$ & $1 \cdot 10^{-3}$ & $2 \cdot 10^{-3}$ & $1 \cdot 10^{-3}$ & $5 \cdot 10^{-3}$ & $2 \cdot 10^{-2}$ & $6 \cdot 10^{-2}$ & $2 \cdot 10^{0}$ \\
\hline
\end{tabular}

Tabela 4.13 Máximos módulos do erro relativo das raízes obtidas através de interpolação da Tabela 4.9, considerando todo o intervalo $c>0$ (ou $c \geq 0,05$ no caso de interpolação de 1aㅡordem de $\beta_{1}$ )

\begin{tabular}{cccccccccc}
\hline \multirow{2}{*}{ Raiz } & \multicolumn{8}{c}{ Ordem da Interpolação } \\
\cline { 2 - 9 } & $1^{\underline{a}}$ & $2^{\underline{a}}$ & $3^{\underline{a}}$ & $4^{\underline{a}}$ & $5^{\underline{a}}$ & $6^{\underline{a}}$ & $7^{\underline{a}}$ & $8^{\underline{a}}$ & $9^{\underline{a}}$ \\
\hline$\beta_{1}$ & $2 \cdot 10^{-2}$ & $5 \cdot 10^{-2}$ & $2 \cdot 10^{-2}$ & $1 \cdot 10^{-2}$ & $9 \cdot 10^{-3}$ & $7 \cdot 10^{-3}$ & $6 \cdot 10^{-3}$ & $5 \cdot 10^{-3}$ & $1 \cdot 10^{-1}$ \\
$\beta_{2}$ & $1 \cdot 10^{-3}$ & $2 \cdot 10^{-4}$ & $8 \cdot 10^{-5}$ & $4 \cdot 10^{-5}$ & $1 \cdot 10^{-5}$ & $4 \cdot 10^{-6}$ & $2 \cdot 10^{-6}$ & $3 \cdot 10^{-6}$ & $4 \cdot 10^{-6}$ \\
$\beta_{3}$ & $2 \cdot 10^{-3}$ & $3 \cdot 10^{-4}$ & $1 \cdot 10^{-4}$ & $7 \cdot 10^{-5}$ & $4 \cdot 10^{-5}$ & $1 \cdot 10^{-5}$ & $6 \cdot 10^{-6}$ & $3 \cdot 10^{-6}$ & $4 \cdot 10^{-6}$ \\
$\beta_{4}$ & $2 \cdot 10^{-3}$ & $5 \cdot 10^{-4}$ & $1 \cdot 10^{-4}$ & $8 \cdot 10^{-5}$ & $5 \cdot 10^{-5}$ & $2 \cdot 10^{-5}$ & $1 \cdot 10^{-5}$ & $5 \cdot 10^{-6}$ & $2 \cdot 10^{-6}$ \\
$\beta_{5}$ & $3 \cdot 10^{-3}$ & $7 \cdot 10^{-4}$ & $1 \cdot 10^{-4}$ & $6 \cdot 10^{-5}$ & $4 \cdot 10^{-5}$ & $2 \cdot 10^{-5}$ & $1 \cdot 10^{-5}$ & $6 \cdot 10^{-6}$ & $3 \cdot 10^{-6}$ \\
$\beta_{6}$ & $3 \cdot 10^{-3}$ & $1 \cdot 10^{-3}$ & $2 \cdot 10^{-4}$ & $5 \cdot 10^{-5}$ & $2 \cdot 10^{-5}$ & $1 \cdot 10^{-5}$ & $8 \cdot 10^{-6}$ & $4 \cdot 10^{-6}$ & $3 \cdot 10^{-6}$ \\
$\beta_{7}$ & $4 \cdot 10^{-3}$ & $1 \cdot 10^{-3}$ & $3 \cdot 10^{-4}$ & $9 \cdot 10^{-5}$ & $2 \cdot 10^{-5}$ & $6 \cdot 10^{-6}$ & $3 \cdot 10^{-6}$ & $2 \cdot 10^{-6}$ & $2 \cdot 10^{-6}$ \\
$\beta_{8}$ & $4 \cdot 10^{-3}$ & $2 \cdot 10^{-3}$ & $4 \cdot 10^{-4}$ & $2 \cdot 10^{-4}$ & $4 \cdot 10^{-5}$ & $1 \cdot 10^{-5}$ & $3 \cdot 10^{-6}$ & $1 \cdot 10^{-6}$ & $1 \cdot 10^{-6}$ \\
$\beta_{9}$ & $5 \cdot 10^{-3}$ & $2 \cdot 10^{-3}$ & $5 \cdot 10^{-4}$ & $2 \cdot 10^{-4}$ & $7 \cdot 10^{-5}$ & $3 \cdot 10^{-5}$ & $9 \cdot 10^{-6}$ & $3 \cdot 10^{-6}$ & $2 \cdot 10^{-6}$ \\
\hline
\end{tabular}




\section{NOVAS EXPRESSÕES APROXIMADAS PARA A PRIMEIRA RAIZ}

\subsection{Uma Expressão que Utiliza Ponto Fixo}

Define-se matematicamente "ponto fixo" de uma função como o ponto que não tem seu valor alterado após a aplicação da função. As raízes da Eq. (1.5) são pontos fixos de várias funções:

$$
\begin{gathered}
f\left(\beta_{1}\right)=\frac{c}{\tan \beta_{1}} \\
f\left(\beta_{1}\right)=\arctan \frac{c}{\beta_{1}} \\
f\left(\beta_{1}\right)=\beta_{1}\left(1-\tan \beta_{1}\right)-c \\
f\left(\beta_{1}\right)=\operatorname{arcsen} \frac{c \cos \beta_{1}}{\beta_{1}}
\end{gathered}
$$

e a substituição dos $\beta_{1}$ que aparecem nestas equações por um valor aproximado da raiz, leva a um outro valor aproximado da raiz que pode ser mais exato.

A segunda função listada acima, $\operatorname{com} c=0,5$, está representada no gráfico da Figura 5.1. Pela Tabela 5.1, obtém-se que para $c=0,5$, a raiz da Eq. (1.5) é $\beta_{1}=0,6533$, valor este que corresponde à abscissa da intersecção desta curva com a bissetriz $y=\beta$. Observa-se que a utilização de um valor aproximado de $\beta_{1}$ menor do que este valor correto, leva a um correspondente $f\left(\beta_{1}\right)$ com valor maior do que o valor exato, e vice-versa.

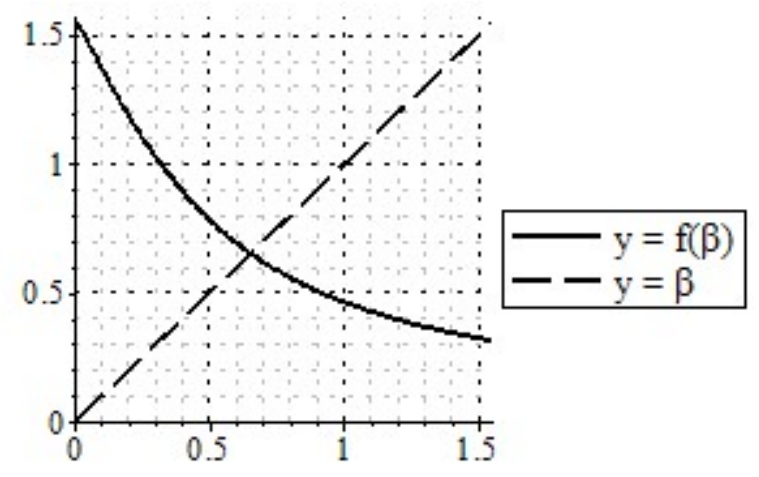

Figura 5.1 Ponto de intersecção correspondente à primeira raiz da Eq. (1.5)

Para qualquer valor de $c, f$ é decrescente, pois: 


$$
\frac{d}{d \beta_{1}} f\left(\beta_{1}\right)=\frac{d}{d \beta_{1}} \arctan \frac{c}{\beta_{1}}=-\frac{c}{\beta_{1}^{2}+c^{2}}<0
$$

e a declividade de $f$ é acentuada quando $c$ tem um valor baixo, pois

$$
\lim _{\beta_{1} \rightarrow 0}\left(\frac{d}{d \beta_{1}} f\left(\beta_{1}\right)\right)=-\frac{1}{c}
$$

Então, se for utilizado um baixo valor aproximado da raiz, pode-se obter um novo valor muito alto, o que acarreta em aumento do valor absoluto do erro, ao invés de diminuição. Se, por outro lado, for utilizado um valor aproximado maior do que o valor exato da raiz, o novo valor calculado será menor do que o valor exato, e novamente o erro poderá aumentar em termos absolutos.

Observando com mais cuidado, verifica-se que a declividade relevante é a da parte da curva que se situa entre o valor aproximado utilizado e o valor exato e, caso ela seja menor do que a unidade, o cálculo da expressão resultará num valor com mais exatidão.

0 valor absoluto da declividade da curva $y=f(\beta)$ no ponto de intersecção com a reta $y=\beta$ é:

$$
\left|\frac{d}{d \beta_{1}} f\left(\beta_{1}\right)\right|_{\beta_{1} \tan \beta_{1}=c}|=|-\left.\frac{c}{\beta_{1}^{2}+c^{2}}\right|_{\beta_{1} \tan \beta_{1}=c}|=| \frac{\sin 2 \beta_{1}}{2 \beta_{1}} \mid \leq 1 \text { para } 0 \leq \beta_{1} \leq \frac{\pi}{2}
$$

Então, sabe-se que obrigatoriamente existirá uma vizinhança deste ponto de intersecção onde a declividade em todos pontos será menor do que a unidade. Portanto, se o valor aproximado estiver suficientemente próximo do valor exato (correspondente ao ponto de intersecção), ele estará numa parte da curva que garantirá que o módulo do erro diminua e o valor calculado seja uma aproximação melhor.

Entre várias combinações averiguadas, um bom resultado foi obtido utilizando esta função discutida com a estimativa da Eq. (2.25), resultando:

$$
\beta_{1} \cong \arctan \frac{c}{\sqrt{\frac{c}{1+\frac{c}{\beta_{1, \infty}^{2}}}}}
$$

que pode ser simplificada para:

$$
\beta_{1} \cong \arctan \sqrt{\mathrm{c}+\frac{4 c^{2}}{\pi^{2}}}
$$

Esta expressão continua satisfazendo a tendência da Eq. (2.12):

$$
\lim _{c \rightarrow 0^{+}}\left(\frac{\arctan \sqrt{c+\frac{4 c^{2}}{\pi^{2}}}}{\sqrt{c}}\right)=1
$$


e continua tendo valor correto quando $c$ tende a infinito:

$$
\lim _{c \rightarrow \infty}\left(\arctan \sqrt{c+\frac{4 c^{2}}{\pi^{2}}}\right)=\frac{\pi}{2}=\beta_{1, \infty}
$$

portanto, seu erro relativo tende a zero quando $c$ tende a zero ou infinito como pode ser visto na Figura 8.7.

Observe-se neste mesmo gráfico, como esta expressão reduziu o erro da expressão original e, portanto, todas as estimativas da Eq. (2.25) estiveram suficientemente próximas das intersecções para que a declividade da curva não ultrapassasse a unidade.

Não é possível se aplicar o mesmo procedimento para a Eq. (1.6) porque, como já apontado anteriormente, apesar de $\sin \beta / \cos \beta$ ser definido $\operatorname{como} \tan \beta$, a razão entre as funções de Bessel $\mathrm{J}_{1}(\beta) / \mathrm{J}_{0}(\beta)$ não é definida como uma nova função e, portanto, não tem inversa.

Repetindo-se o mesmo procedimento para a primeira raiz da Eq. (1.7), obtém-se:

$$
\beta_{1} \cong \operatorname{arccot}\left(\frac{1-c}{\pi} \sqrt{1+\frac{\pi^{2}}{3 c}}\right)
$$

a qual, por cálculos análogos aos já mostrados, tem erro relativo tendendo a zero quando $c$ tende a zero ou infinito, como pode ser visto na Figura 8.9.

As expressões mostradas nas Eqs. (5.1) e (5.2) são bastante simples por sequer incluírem parâmetros de ajuste. Elas consistem nas primeiras novas expressões desenvolvidas neste trabalho, e estão identificadas por "Ponto Fixo".

\subsection{Coeficientes Ótimos para as Expressões Já Desenvolvidas}

Várias expressões obtidas em outros trabalhos são casos particulares da expressão:

$$
\beta_{1} \cong\left(p c^{s}+q\right)^{\frac{1}{r}}
$$

onde $p, q, r$ e $s$ são constantes. É muito interessante observar nos trabalhos que mostram como as expressões foram obtidas, que isto ocorreu por diferentes deduções.

A expressão de Luikov (1968) mostrada na Eq. (2.14) e obtida através de gráficos logarítmicos:

$$
\beta_{1} \cong \frac{\beta_{1, \infty}}{\sqrt{1+\frac{A}{c^{k}}}}=\left(A \beta_{1, \infty}^{-2} c^{-k}+\beta_{1, \infty}^{-2}\right)^{-\frac{1}{2}}
$$


corresponde ao caso em que $p=A \beta_{1, \infty}^{r}, s=-k, q=\beta_{1, \infty}^{r}, r=-2$.

A expressão de Yovanovich (1996) mostrada na Eq. (2.19) e obtida a partir da relação empírica de Churchill e Usagi (1972):

$$
\beta_{1} \cong \frac{\beta_{1, \infty}}{\left(1+\left(\frac{\beta_{1, \infty}}{\sqrt{m c}}\right)^{k}\right)^{\frac{1}{k}}}=\left(m^{-\frac{k}{2}} c^{-\frac{k}{2}}+\beta_{1, \infty}^{-k}\right)^{-\frac{1}{k}}
$$

corresponde ao caso no qual $p=m^{r / 2}, s=r / 2, q=\beta_{1, \infty}^{r}, r=-k$.

A expressão de Ostrogorsky e Mikic (2008) mostrada na Eq. (2.25) e obtida através de séries de potências:

$$
\beta_{1} \cong \sqrt{\frac{m c}{1+\frac{m c}{\beta_{1, \infty}^{2}}}}=\left(\frac{1}{m} c^{-1}+\beta_{1, \infty}^{-2}\right)^{-1 / 2}
$$

corresponde ao caso no qual $p=m^{r / 2}, s=r / 2, q=\beta_{1, \infty}^{r}, r=-2$.

A expressão de Stevens e Luck (1999) mostrada na Eq. (2.20) para a primeira raiz da Eq. (1.5):

$$
\beta_{1} \cong \frac{\pi}{4}+\frac{\pi}{4} \frac{c-\frac{\pi}{4}}{c+\frac{\pi}{4}}=\left(\frac{1}{2} c^{-1}+\frac{2}{\pi}\right)^{-1}
$$

corresponde ao caso no qual $p=1 / 2, s=-1, q=\beta_{1, \infty}^{r}, r=-1$; a expressão mostrada na Eq. (2.21) para a primeira raiz da Eq. (1.6):

$$
\beta_{1} \cong \frac{3 \pi}{8}+\frac{3 \pi}{8} \frac{c-\frac{\pi}{2}}{c+\frac{\pi}{2}}=\left(\frac{2}{3} c^{-1}+\frac{4}{3 \pi}\right)^{-1}
$$

corresponde ao caso no qual $p=2 / 3, s=-1, q \cong \beta_{1, \infty}^{r}, r=-1$; e a expressão mostrada na Eq. (2.22) para a primeira raiz da Eq. (1.7):

$$
\beta_{1} \cong \frac{\pi}{2}+\frac{\pi}{2} \frac{c^{0,676}-\frac{\sqrt{\pi}}{2}}{c^{0,676}+\frac{\sqrt{\pi}}{2}}=\left(\frac{1}{2 \sqrt{\pi}} c^{-0,676}+\frac{1}{\pi}\right)^{-1}
$$

corresponde ao caso no qual $p=1 /(2 \sqrt{\pi}), s=-0,676, q=\beta_{1, \infty}^{r}, r=-1$.

A expressão de Ostrogorsky e Mikic (2009) mostrada na Eq. (2.26) para $0 \leq c<2$ :

$$
\beta_{1} \cong \sqrt{\frac{m c}{1+\frac{c}{m+2}}}=\left(\frac{1}{m} c^{-1}+\frac{1}{m(m+2)}\right)^{-1 / 2}
$$

corresponde ao caso qual $p=m^{r / 2}, s=r / 2, q=1 /(m(m+2)), r=-2$, e a expressão para $c \geq 2$ :

$$
\beta_{1} \cong \beta_{1, \infty} \frac{c}{c+a}=\left(a \beta_{1, \infty}^{-1} c^{-1}+\beta_{1, \infty}^{-1}\right)^{-1}
$$


corresponde ao caso no qual $p=a \beta_{1, \infty}^{-1}, q=\beta_{1, \infty}^{r}, s=-1, r=-1$.

A expressão de Ostrogorsky (2010) mostrada na Eq. (2.27) para $0 \leq c \leq 0,1$ e obtida da tendência dada na Eq. (2.12):

$$
\beta_{1} \cong \sqrt{m c}=\left(m^{1 / 2} c^{1 / 2}+0\right)^{1}
$$

corresponde ao caso no qual $p=m^{r / 2}, s=r / 2, q=0, r=1$, e a expressão para $c>0,1$ :

$$
\beta_{1} \cong \frac{\beta_{1, \infty}}{\sqrt{1+\frac{a}{c^{k}}}}=\left(a \beta_{1, \infty}^{-2} c^{-k}+\beta_{1, \infty}^{-2}\right)^{-\frac{1}{2}}
$$

corresponde ao caso no qual $p=a \beta_{1, \infty}^{-2}, s=-k, q=\beta_{1, \infty}^{r}, r=-2$. Por fim, a expressão mostrada na Eq. (2.28):

$$
\beta_{1} \cong \frac{\beta_{1, \infty}}{\left(1+\frac{a}{c^{k}}\right)^{d}}=\left(a \beta_{1, \infty}^{-1 / d} c^{-k}+\beta_{1, \infty}^{-1 / d}\right)^{-d}
$$

corresponde ao caso no qual $p=a \beta_{1, \infty}^{-1 / d}, s=-k, q=\beta_{1, \infty}^{r}, r=-1 / d$.

Então, nosso objetivo será o de encontrar os melhores parâmetros para essa expressão. Inicialmente, a particularizaremos para que ela tenha os valores corretos quando $c$ tende a zero ou a infinito:

$$
\begin{gathered}
\lim _{c \rightarrow 0} \beta_{1}=\lim _{c \rightarrow 0}\left(p c^{s}+q\right)^{\frac{1}{r}}=0=\beta_{1,0} \\
\lim _{c \rightarrow \infty} \beta_{1}=\lim _{c \rightarrow \infty}\left(p c^{s}+q\right)^{\frac{1}{r}}=\beta_{1, \infty} \rightarrow s<0, q=\beta_{1, \infty}^{r}
\end{gathered}
$$

Observe-se que este valor de $q$ foi utilizado em todas expressões, exceto naquelas que não abrangem o final do intervalo e, portanto, não precisam tender a $\beta_{1, \infty}$.

Assim, a expressão particularizada passa a ser:

$$
\beta_{1} \cong\left(p c^{s}+\beta_{1, \infty}^{r}\right)^{\frac{1}{r}}
$$

onde há três parâmetros que devem ser calculados para que sua curva se ajuste a curva exata. Esta expressão é a mesma utilizada por Ostrogorsky (2010) na Eq. (2.28), onde há três parâmetros equivalentes calculados através de um critério de ajuste que não foi informado.

O Método dos Mínimos Quadrados é muito utilizado para ajuste de curvas, porém, ele não deve ser aplicado ao presente problema porque ele leva a melhor função de ajuste quando os pontos ou a curva a ser aproximada tem erro com distribuição normal.

No presente caso, a curva a ser aproximada não tem erro, e o objetivo é que o desvio relativo máximo desta expressão seja o menor possível, ou seja, ela deve ser uma aproximação MiniMax, também chamada de aproximação de Chebyshev (POWELL, 1981). 
A obtenção de aproximações MiniMax não é um processo considerado elementar, e será discutido como se fazer isso ainda neste capítulo, apenas adiantando que os valores obtidos para que esta expressão se ajuste a função exata correspondente à primeira raiz da Eq. (1.7) leva à curva mostrada na Figura 5.2. Observe-se que o menor erro máximo é obtido quando os picos atingem mesmo valor, ou seja, o desbalanceamento deles causa um aumento do erro máximo.

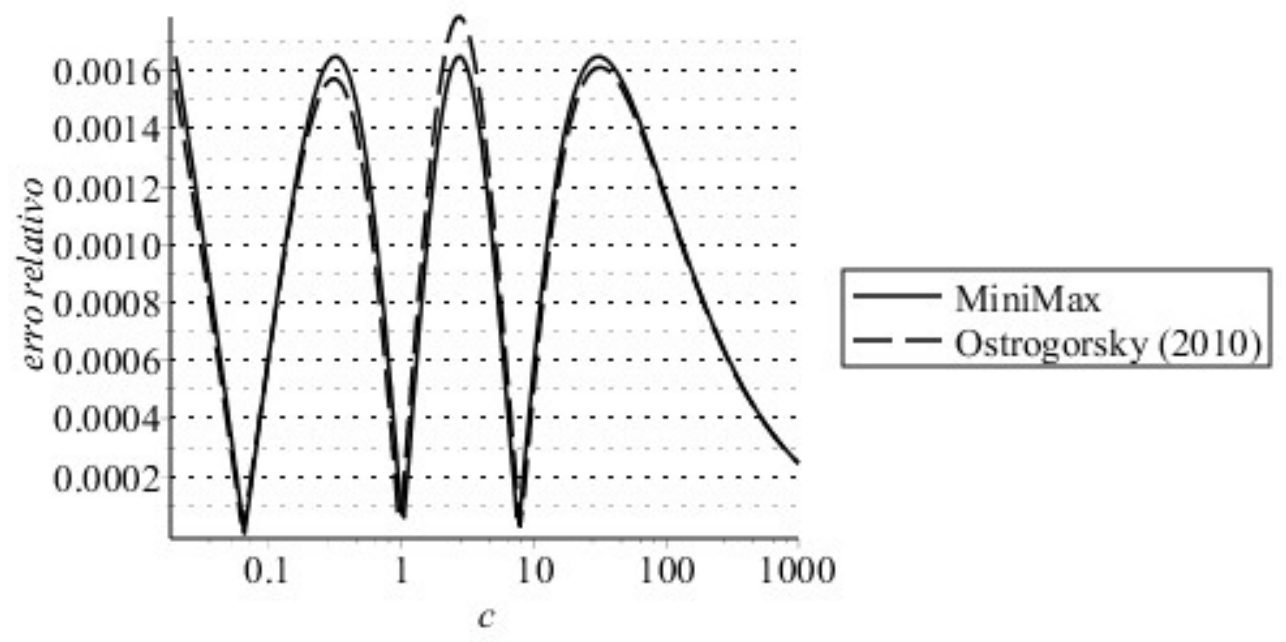

Figura 5.2 Erro relativo dos valores obtidos pela Eq. (2.28) de Ostrogorsky (2010), e dos valores obtidos pela mesma equação com parâmetros calculados pelo critério MiniMax

Porém, ainda não será utilizada essa expressão, porque se considera inconveniente que ela não tenha a tendência da Eq. (2.12) e, portanto, leve a valores de erro proibitivos fora do intervalo de ajuste. Estas raízes são utilizadas em métodos válidos para qualquer $c$, então, a utilização de uma expressão que restrinja os possíveis valores de $c$ acabariam por restringir a validade das equações.

Assim, particularizaremos ainda mais essa expressão para que ela tenha a tendência da Eq. (2.12):

$$
\lim _{c \rightarrow 0^{+}} \frac{\left(p c^{s}+\beta_{1, \infty}^{r}\right)^{\frac{1}{r}}}{\sqrt{m c}}=\lim _{c \rightarrow 0^{+}}\left(p \frac{c^{s-\frac{r}{2}}}{m^{\frac{r}{2}}}+\left(\frac{\beta_{1, \infty}^{2}}{m c}\right)^{\frac{r}{2}}\right)^{\frac{1}{r}}=1
$$

ou seja:

$$
s=\frac{r}{2}<0 \text { e } \frac{p}{m^{\frac{r}{2}}}=1
$$

e a expressão resultante é

$$
\beta_{1} \cong\left((m c)^{\frac{r}{2}}+\beta_{1, \infty}^{r}\right)^{\frac{1}{r}}
$$


onde $r<0$ é o parâmetro de ajuste.

Esta expressão é a mesma que Yovanovich (1996) obteve a partir da expressão de Churchill e Usagi (1972), e ajustou por algum critério não relatado. Utilizaremos, então, o conceito da aproximação MiniMax para ajustar esta expressão aos valores exatos através da determinação do parâmetro $r$.

Pesquisando os sistemas algébricos computacionais modernos, observa-se que eles procuram os parâmetros MiniMax apenas de funções racionais, e não se encontrou algum que pudesse ser utilizado para se buscar os parâmetros desta expressão. Desenvolvemos um modo para se chegar a estes parâmetros através de gráficos que será apresentado agora.

O erro relativo desta expressão plotado em função da variável independente $c$ e do parâmetro $r$ para o caso das raízes da Eq. (1.5), está no gráfico tridimensional da Figura 5.3.

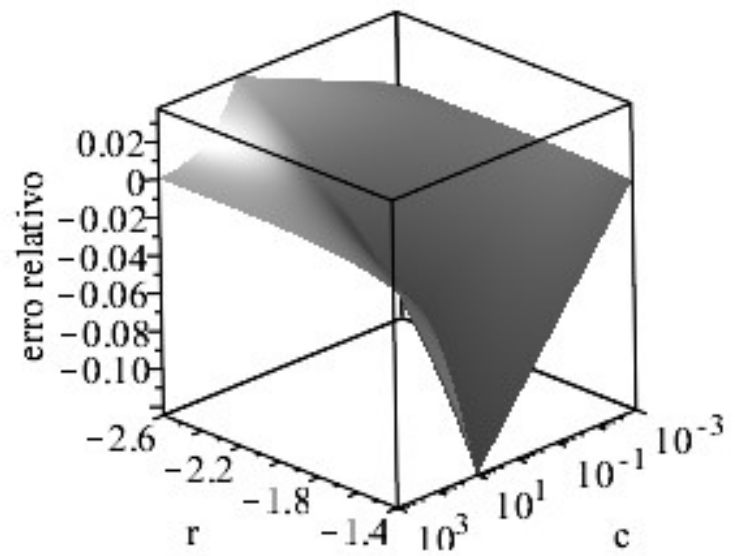

Figura 5.3 Erro relativo das raízes da Eq. (1.5) calculadas pela Eq. (5.4)

Este gráfico pode ser rotacionado para que o eixo de $c$ fique perpendicular ao plano da figura, e o valor ótimo do parâmetro $r$ se torne evidente pelo "afunilamento" na área no ponto em que o módulo do erro relativo é mínimo (Figura 5.4).

É claro que este "afunilamento" não existiria caso a forma da equação não fosse conveniente para a curva a ser ajustada quando, então, não haveria um valor do parâmetro que levaria a valores baixos de erro.

Por este gráfico, pode-se ver que o valor ótimo se encontra entre -2 e -2,2, e um novo gráfico é gerado com os valores de $r$ limitados a este intervalo. Consecutivos refinamentos levam ao valor de $r$ com tanta exatidão quanto se queira. 


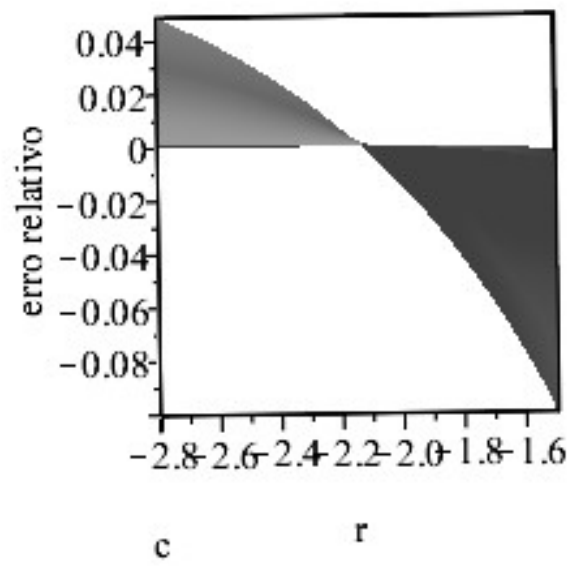

Figura 5.4 Erro relativo das raízes da Eq. (1.5) calculadas pela Eq. (5.4) com o eixo de c perpendicular à figura

Durante esse processo, os gráficos tridimensionais podem ser substituídos por gráficos bidimensionais. Três seções transversais da superfície mostrada na Figura 5.3, correspondem às curvas mostradas na Figura 5.5.

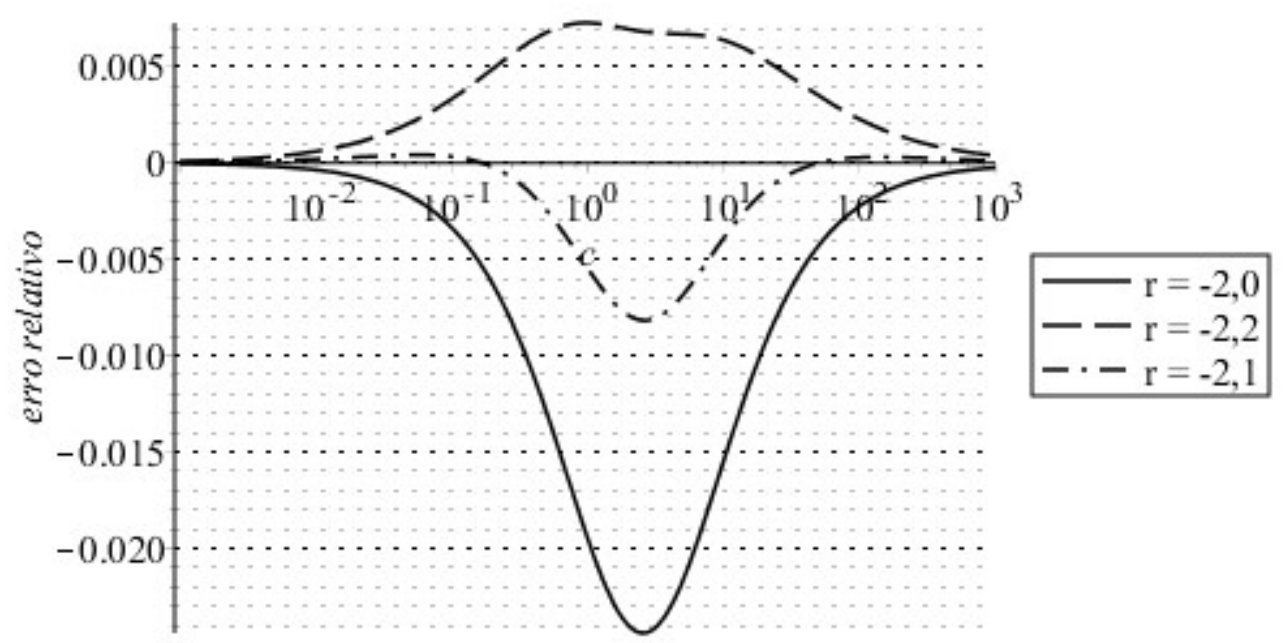

Figura 5.5 Erro relativo das raízes da Eq. (1.5) calculadas pela Eq. (5.4) para três valores do parâmetro de ajuste r

A curva correspondente a $r=-2$ se encontra onde a superfície da Figura 5.3 passa por valores negativos do erro relativo, enquanto que a curva correspondente a $r=-2,2$ se encontra na parte posterior da superfície, que passa por valores de erro relativo positivo.

A curva correspondente aos menores valores de erro se encontra numa parte da superfície com um valor de $r$ intermediário a estes dois. Então, utilizando algo que lembra o método da bisseção 
de busca de raízes, traçou-se a curva correspondente a $r=-2,1$. Esta curva também tem um ponto de erro negativo com valor absoluto maior, o que significa que o valor de $r$ que leva ao erro mínimo se encontra entre $-2,1$ e $-2,2$.

Uma nova curva com $r=-2,15$ é traçada, e essa análise é repetida. Além de observar o erro decrescente, durante essa sequência de obtenções do valor do parâmetro $r$, também se observou que a forma das curvas se modificou sucessivamente de maneira que o máximo módulo do erro relativo negativo convergiu para o mesmo valor do erro relativo positivo mínimo, o que é previsto pela teoria do MiniMax para funções racionais. Isto pode ser verificado no gráfico final da Figura 5.6.

Comparando-se a Figura 5.6 com a Figura 5.2, nota-se que o aumento do erro máximo foi bastante baixo, e a incorporação das tendências corretas justificam-se plenamente. Verifica-se também que o maior arredondamento que não produz perda significativa de exatidão é $r=-2,14$.

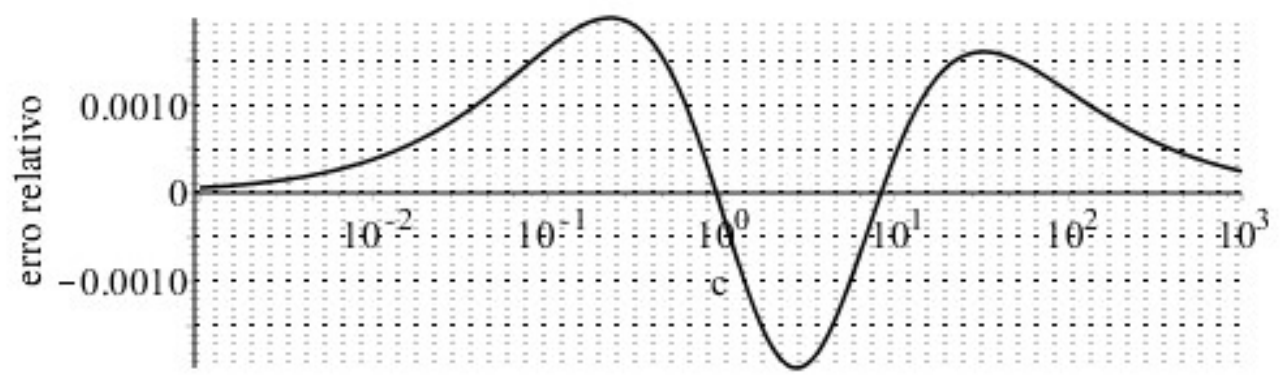

Figura 5.6 Erro relativo das raízes da Eq. (1.5) calculadas pela Eq. (5.4) para o valor ótimo do parâmetro de ajuste r

Para as Eqs. (1.6) e (1.7), os gráficos iniciais são bastante semelhantes a estes da Eq. (1.5) (Figura 5.7 e Figura 5.8, respectivamente). A partir deles repetiu-se o procedimento acima, inclusive com o arredondamento dos valores finais até o limite que permitia não haver perda significativa de exatidão, obtendo-se $r=-2,14,-2,25$ e $-2,34$ para as Eqs. (1.5), (1.6) e (1.7), respectivamente.

Os erros relativos das raízes calculadas pela Eq. (5.4) utilizando esses valores do parâmetro $r$ que correspondem ao MiniMax estão na Figura 8.10, Figura 8.11 e Figura 8.12, para as raízes das Eqs. (1.5), (1.6) e (1.7), respectivamente. Ela está identificada como "Yovanovich MiniMax".

A simetria dos pontos extremos não está exata nestas figuras devido ao arredondamento dos parâmetros, e os pontos de mínimo também se situam para cima, devido a se utilizar módulos do erro relativo nestes gráficos. A comparação destes gráficos com as expressões dos trabalhos aqui discutidos corrobora as opções escolhidas. 


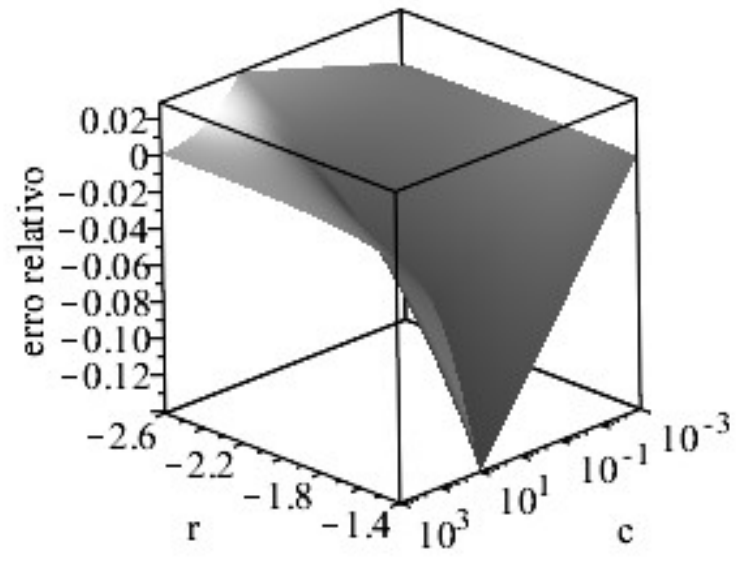

Figura 5.7 Erro relativo das raízes da Eq. (1.6) calculadas pela Eq. (5.4)

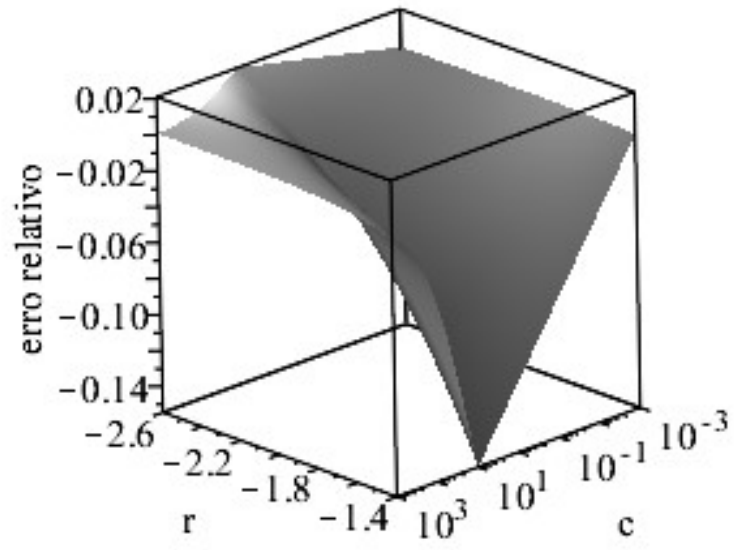

Figura 5.8 Erro relativo das raízes da Eq. (1.7) calculadas pela Eq. (5.4)

\subsection{Uma Expressão Algébrica}

O ajuste por expressões racionais é muito utilizado porque elas são simples, abrangem formas muito variadas de curvas, não são muito oscilantes, e podem ter assíntotas verticais. A ordem de 2x2 geralmente é suficiente para um bom ajuste de curvas como a do presente trabalho, que podem ser consideradas "bem-comportadas" e, então:

$$
\beta_{1} \cong \frac{A_{0}+A_{1} c+A_{2} c^{2}}{B_{0}+B_{1} c+c^{2}}
$$

Para essa expressão ter o valor correto $\beta_{1}=0$ em $c=0$, deve ser utilizado $A_{0}=0$, e para ela tender ao valor correto $\beta_{1, \infty}$ quando $c \rightarrow \infty$, deve ser utilizado $A_{2}=\beta_{1, \infty}$. Então: 


$$
\beta_{1} \cong \frac{A_{1} c+\beta_{1, \infty} c^{2}}{B_{0}+B_{1} c+c^{2}}
$$

Porém, para ela ter a tendência dada pela Eq. (2.12), seria necessário que o seguinte limite resultasse em valor unitário:

$$
\lim _{c \rightarrow 0} \frac{\frac{A_{1} c+\beta_{1, \infty} c^{2}}{B_{0}+B_{1} c+c^{2}}}{\sqrt{m c}}=\lim _{c \rightarrow 0} \frac{\sqrt{c}\left(A_{1}+\beta_{1, \infty} c\right)}{\sqrt{m}\left(B_{0}+B_{1} c+c^{2}\right)}
$$

Se $B_{0}=0$, então este limite é infinito, e se $B_{0} \neq 0$, então esse limite é nulo, ou seja, o valor unitário para o limite e, portanto, a tendência correta, nunca pode ser obtida. É possível se modificar essa expressão para que ela possa ter essa tendência para:

$$
\beta_{1} \cong \frac{A_{1} \sqrt{c}+\beta_{1, \infty} c^{2}}{B_{0}+B_{1} c+c^{2}}
$$

Assim:

$$
\lim _{c \rightarrow 0} \frac{\frac{A_{1} \sqrt{c}+\beta_{1, \infty} c^{2}}{B_{0}+B_{1} c+c^{2}}}{\sqrt{m c}}=\lim _{c \rightarrow 0} \frac{A_{1}+\beta_{1, \infty} c^{\frac{3}{2}}}{\sqrt{m}\left(B_{0}+B_{1} c+c^{2}\right)}=\lim _{c \rightarrow 0} \frac{A_{1}}{\sqrt{m} B_{0}}=1 \rightarrow A_{1}=\sqrt{m} B_{0}
$$

Então, uma expressão com as tendências corretas é:

$$
\beta_{1} \cong \frac{A \sqrt{m c}+\beta_{1, \infty} c^{2}}{A+B c+c^{2}}
$$

onde os parâmetros $A$ e $B$ devem ser determinados por ajuste.

$\mathrm{Na}$ seção anterior, havia apenas um parâmetro a ser determinado, então um gráfico tridimensional com o erro relativo em função do parâmetro e da variável independente $c$ foi utilizado. Agora há dois parâmetros, então não há como se fazer uma superfície correspondente ao erro. Então, seguiremos diretamente para os gráficos bidimensionais com as curvas de erro, sabendo que elas correspondem a algo como uma intersecção de uma superfície $n$-dimensional.

Encontrou-se lógica nas "iterações gráficas". Os valores do erro diminuem conforme os picos e vales tornam-se simétricos. Enquanto que no caso anterior, que correspondia a um único parâmetro, apenas um pico e um vale tornavam-se simétricos, aqui, há três pontos extremos tornando-se simétricos ao mesmo tempo.

Além disso, quanto maior a potência de $c$ que um parâmetro multiplica, maior a influência para a parte direita das curvas, ou seja, aumentando um desses parâmetros, o lado direito das curvas sobe, e vice-versa. Já os parâmetros que multiplicam potências menores de $c$ atuam de maneira contrária, variando o lado esquerdo das curvas. Quando um parâmetro multiplica toda a expressão, ele simplesmente desloca a curva toda. 
O menor erro máximo encontrado é de 0,92\% e correspondeu aos valores dos parâmetros que levaram a curva de erro mostrada na Figura 5.9.

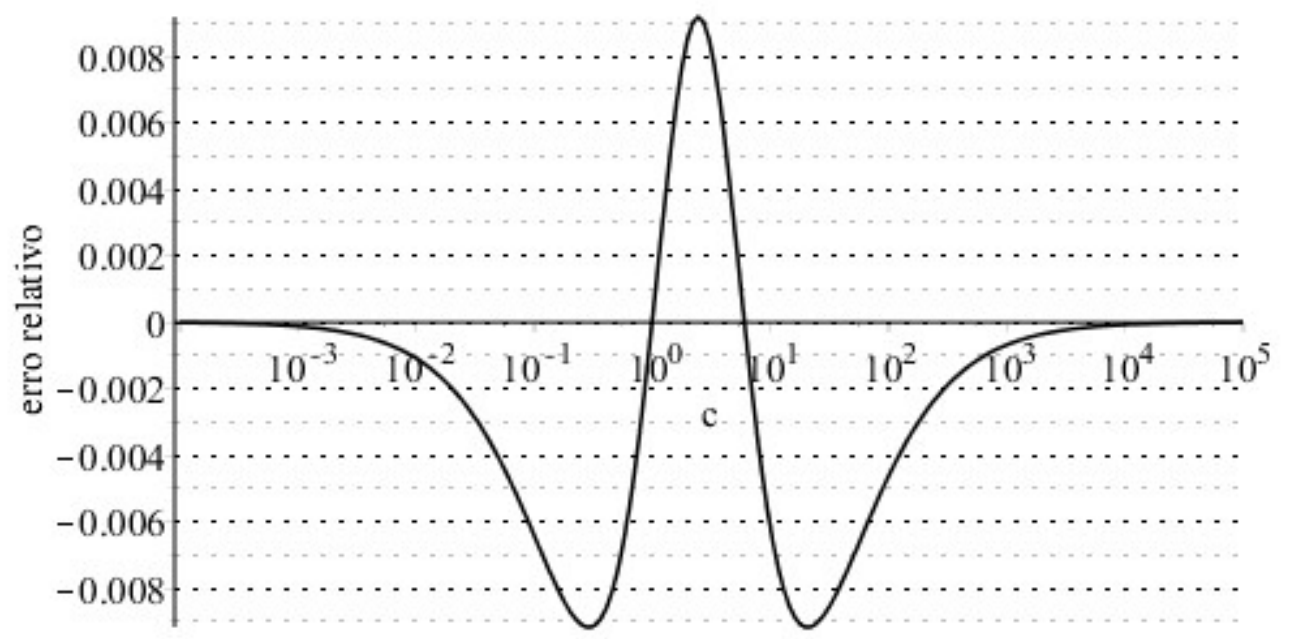

Figura 5.9 Erro relativo das raízes da Eq. (1.5) calculadas pela primeira forma da expressão algébrica

Os parâmetros de expressões MiniMax podem ser obtidos pelo algoritmo de trocas de Remez (POWELL, 1981) porém, sua convergência está provada apenas para funções racionais e esta expressão acima contém um termo $\operatorname{com} c^{1 / 2}$, então ela não é racional, pois as expressões racionais apenas podem ter potências inteiras da variável independente (ZHIZHCHENKO, 2011).

Será investigado o resultado obtido ao se aplicar este algoritmo. Devido a haver dois parâmetros para serem calculados, o algoritmo inicia com um conjunto de 2+1 pontos arbitrários da curva exata, que foram escolhidos serem os de abscissas [0,1 110$]$. A curva correspondente a esta expressão precisa passar com uma proximidade destes pontos que leve a um erro relativo de mesmo módulo e com sinais alternados por estes pontos.

0 cálculo desta curva é a parte mais crítica, por se tratar de um sistema de equações não lineares, e que talvez não tenha solução. Porém, foi possível encontrá-la com $A=6,767$ e $B=1,877$, e com o módulo do erro relativo dado por $h=0,004963$.

O passo seguinte é o que consome mais tempo computacional. Comparando-se a curva que utiliza estes valores de parâmetros com a curva exata, encontra-se que o erro relativo máximo se encontra no ponto com abscissa $c=2,380$ e tem um valor de $H=+0,01439$.

A eficiência desse algoritmo é notável. Observe-se que estes parâmetros encontrados $A=$ 6,767 e $B=1,877$ já levaram ao erro de 1,439\%, tudo isso já na primeira iteração, através da resolução de um único sistema de equações. E as próximas etapas diminuirão este erro. 
0 ponto de abscissa $c=2,380$ encontra-se entre o segundo e o terceiro pontos arbitrários iniciais, e deve substituir um deles, o que mantiver a alternância dos sinais. Fazendo-se isso, obtémse o novo conjunto de pontos com abscissas [0,1 2,380 10] sobre o qual o sistema de equações é novamente resolvido, novos valores de parâmetros são obtidos $A=5,733$ e $B=1,779$ com os quais, a expressão passa a ter erro máximo de 1,033\%.

Repetindo-se sucessivamente esses passos, o algoritmo prevê que $h$ deve aumentar e $H$ deve diminuir em módulo até tornarem-se iguais quando, então, a convergência é alcançada, e os parâmetros ótimos são encontrados. Isto foi obtido, e o erro relativo máximo atingiu o valor $h=|H|=$ 0,9183\% (Tabela 5.1).

Quando se trata de funções racionais, demonstra-se que a sequência dos valores do erro é estritamente decrescente, o que não foi observado no caso desta expressão. Porém, encontrou-se exatamente os mesmos resultados que já haviam sido obtidos através das iterações gráficas.

Repetindo-se esse procedimento para a Eq. (1.6) obtém-se o erro relativo máximo $h=|H|=$ 0,009718 (Tabela 5.2), e para a Eq. (1.7) obtém-se o erro relativo máximo $h=|H|=0,01001$ (Tabela 5.3). Esses resultados foram confirmados através das iterações gráficas, e eles realmente correspondem ao ajuste MiniMax.

Para não se utilizar parâmetros com muitos algarismos decimais, procurou-se os valores arredondados que fizessem com que o erro não aumentasse significativamente. Então, por exemplo, para se arredondar o $B=1,929$ para $B=1,9$ na Eq. (1.6) foi necessário variar o $A=7,863$ para $A=$ 7,6. Assim, para a Eq. (1.5):

$$
\beta_{1} \cong \frac{6 \sqrt{c}+1,57 c^{2}}{6+1,8 c+c^{2}}
$$

para a Eq. (1.6):

$$
\beta_{1} \cong \frac{7,7 \sqrt{2 c}+2,4 c^{2}}{7,7+1,9 c+c^{2}}
$$

e para a Eq. (1.7):

$$
\beta_{1} \cong \frac{9,3 \sqrt{3 c}+3,14 c^{2}}{9,3+2 c+c^{2}}
$$

onde os erros relativos máximos permanecem na ordem de $0,9 \%$ a $1 \%$.

Porém, essas expressões não serão utilizadas sem antes se investigar se não é possível encontrar outras melhores. Devido a ter sido possível se chegar a valores dos parâmetros de uma expressão com um dos termos com potência não inteira, foi iniciada uma tentativa mais ousada, que é a de se procurar expressões com mais potências não inteiras. 
Tabela 5.1 Resultados do algoritmo de troca de Remez para a primeira raiz da Eq. (1.5) calculada pela primeira forma da expressão algébrica

\begin{tabular}{cccccc}
\hline Abscissas & $A$ & $B$ & $h$ & $c$ & $H$ \\
\hline$[0,1110]$ & 6,767 & 1,877 & 0,004963 & 2,380 & 0,01439 \\
{$[0,12,38010]$} & 5,773 & 1,779 & 0,006946 & 0,3129 & $-0,01033$ \\
{$[0,31292,38010]$} & 6,630 & 1,900 & 0,008148 & 19,36 & $-0,01091$ \\
{$[0,31292,38019,36]$} & 5,976 & 1,795 & 0,009163 & 0,2897 & $-0,009191$ \\
{$[0,28972,38019,36]$} & 5,982 & 1,796 & 0,009174 & 20,60 & $-0,009191$ \\
{$[0,28972,38020,60]$} & 5,978 & 1,795 & 0,009180 & 2,444 & 0,009191 \\
{$[0,28972,44420,60]$} & 5,978 & 1,795 & 0,009183 & 0,2895 & $-0,009183$ \\
\hline
\end{tabular}

Tabela 5.2 Resultados do algoritmo de troca de Remez para a primeira raiz da Eq. (1.6) calculada pela primeira forma da expressão algébrica

\begin{tabular}{cccccc}
\hline Abscissas & $A$ & $B$ & $h$ & $c$ & $H$ \\
\hline$[0,1110]$ & 10,72 & 2,213 & 0,003891 & 2,776 & 0,01610 \\
{$[0,12,77610]$} & 7,550 & 1,914 & 0,006754 & 0,3679 & $-0,01109$ \\
{$[0,36792,77610]$} & 9,603 & 2,150 & 0,008074 & 21,71 & $-0,01250$ \\
{$[0,36792,77621,71]$} & 7,855 & 1,928 & 0,009698 & 0,3370 & $-0,009735$ \\
{$[0,33702,77621,68]$} & 7,869 & 1,930 & 0,009712 & 23,00 & 0,009728 \\
{$[0,33702,77623,00]$} & 7,863 & 1,929 & 0,009718 & 2,788 & 0,009719 \\
{$[0,33702,78823,00]$} & 7,863 & 1,929 & 0,009718 & 0,3368 & $-0,009718$ \\
\hline
\end{tabular}

Tabela 5.3 Resultados do algoritmo de troca de Remez para a primeira raiz da Eq. (1.7) calculada pela primeira forma da expressão algébrica

\begin{tabular}{cccccc}
\hline Abscissas & $A$ & $B$ & $h$ & $c$ & $H$ \\
\hline$[0,10001,00010,00]$ & 18,41 & 2,830 & 0,002692 & 3,264 & 0,01633 \\
{$[0,10003,26410,00]$} & 9,232 & 2,020 & 0,006506 & 0,4078 & $-0,01143$ \\
{$[0,40783,26410,00]$} & 13,43 & 2,432 & 0,007636 & 24,14 & $-0,01398$ \\
{$[0,40783,26424,14]$} & 9,565 & 2,025 & 0,009971 & 3,040 & 0,01006 \\
{$[0,40783,04024,14]$} & 9,566 & 2,026 & 0,009992 & 0,3719 & 0,01003 \\
{$[0,37193,04024,14]$} & 9,588 & 2,028 & 0,01001 & 24,83 & 0,01001 \\
\hline
\end{tabular}

Para que as tendências permaneçam corretas, a forma que será utilizada é:

$$
\beta_{1} \cong \frac{A \sqrt{m c}+\beta_{1, \infty} c^{k}}{A+B c+c^{k}}
$$

Desta vez, infelizmente não foi possível a aplicação do Algoritmo de Remez com a inclusão da potência como incógnita adicional porque não se encontrou solução para o sistema de equações resultante, mesmo usando os sistemas algébricos computacionais mais modernos. 
O modo utilizado, então, foi um tanto trabalhoso, e consistiu em se testar novas potências uma a uma, recalculando-se as tabelas acima para cada novo caso, e caminhando na direção que levava a melhores resultados.

Apesar de trabalhoso, não há qualquer inovação nesse procedimento. Porém, durante as iterações chegou-se a uma situação nova. Com um conjunto de abscissas de [0,5 3 10], com sinais $[+-+]$, o sistema de equações levou a $c=74$, que corresponde a erro negativo. Então, o algoritmo prevê que se deve substituir uma das abscissas por este valor. Devido a este valor de $c$ ser maior do que todos os três valores do conjunto de abscissas, o único ponto que poderia ser substituído é o de valor $c=10$, porém os sinais passariam a ser $[+--$, o que contradiz os fundamentos do algoritmo, porque eles precisariam se manter alternados.

Foi possível encontrar o caminho correto, e não se tratava de uma simples substituição. 0 valor de $c=74$ precisa ser o último, e ele tem erro negativo. Então o novo conjunto de abscissas deve ser [3 1074$]$.

Este procedimento levou a convergência e se chegou aos mesmos valores em termos absolutos para os picos e vales da curva de erro. Foi confirmado que o resultado é uma MiniMax através das iterações gráficas. Essa substituição com deslocamento indica uma possível generalização do algoritmo de Remez.

Para a primeira equação, encontrou-se que os melhores resultados ocorrem quando as potências se situam entre 2,45 e 2,55. Não há grandes diferenças de resultados com variações no segundo dígito decimal das potências, então o valor 2,5 foi escolhido para a nova potência.

Para as demais equações ocorreram exatamente as mesmas situações, e as melhores potências foram as de valor 2,6 e 2,8. Assim, obteve-se para a Eq. (1.5):

$$
\beta_{1} \cong \frac{57 \sqrt{c}+1,57 c^{2,5}}{57+10,15 c+c^{2,5}}
$$

para a Eq. (1.6):

$$
\beta_{1} \cong \frac{136 \sqrt{2 c}+2,405 c^{2,6}}{136+18,6 c+c^{2,6}}
$$

e para a Eq. (1.7):

$$
\beta_{1} \cong \frac{416 \sqrt{3 c}+3,14 c^{2,8}}{416+45,6 c+c^{2,8}}
$$

com erros máximos realmente menores: $0,25 \%$ a 0,4\% em módulo.

Estes resultados de erros começam a se tornar muito interessantes, e a questão agora passa a ser sobre qual a forma ideal de expressão. Não há como se escolher formas arbitrárias e testar uma a uma, porque há uma infinidade de possibilidades. 
Para que uma expressão que corresponda a uma razão com potências de $c$ ter valor correto quando $c$ tende a infinito, é necessário que a maior potência do numerador seja igual a maior potência do denominador e que a razão de seus parâmetros seja igual a $\beta_{1, \infty}$.

Para que esta expressão tenha a tendência dada pela Eq. (2.12), é necessário que a menor potência do numerador seja $1 / 2$, a menor potência do denominador seja zero, e a razão entre seus parâmetros seja $m$.

Contudo, estas condições ainda são satisfeitas por uma infinidade de formas. Felizmente, ao final deste trabalho, obteve-se como caso particular de uma expressão válida para todas as raízes da Eq. (1.5), a seguinte expressão:

$$
\beta_{1} \cong \frac{\sqrt{c^{2}+c}}{2 c / \pi+1}
$$

que possui erro máximo de $0,5 \%$.

Este resultado é um tanto surpreendente porque esta expressão é tão simples que nem mesmo possui um parâmetro de ajuste, e isto nos fornece indicação de uma forma mais geral que pode ser investigada:

$$
\beta_{1} \cong \frac{\left(r^{\frac{1}{q}}(m c)^{\frac{1}{2 q}}+\beta_{1, \infty}^{\frac{1}{q}} c^{\frac{p}{q}}\right)^{q}}{r+c^{p}}
$$

Observe-se como o parâmetro com maior potência no numerador e no denominador levam ao resultado exato quando $c$ tende a infinito:

$$
\lim _{c \rightarrow \infty} \frac{\left(r^{\frac{1}{q}}(m c)^{\frac{1}{2 q}}+\beta_{1, \infty}^{\frac{1}{q}} c^{\frac{p}{q}}\right)^{q}}{r+c^{p}}=\lim _{c \rightarrow \infty} \frac{\left(\beta_{1, \infty}^{\frac{1}{q}} c^{\frac{p}{q}}\right)^{q}}{c^{p}}=\beta_{1, \infty}
$$

e como os parâmetros da menor potência leva a tendência dada pela Eq. (2.12):

$$
\lim _{c \rightarrow 0} \frac{\left(r^{\frac{1}{q}}(m c)^{\frac{1}{2 q}}+\beta_{1, \infty}^{\frac{1}{q}} c^{\frac{p}{q}}\right)^{q}}{r+c^{p}}=\lim _{c \rightarrow 0} \frac{\left(r^{\frac{1}{q}}(m c)^{\frac{1}{2 q}}\right)^{q}}{r}=1
$$

Surpreendentemente, os sistemas algébricos computacionais que não puderam resolver o sistema de equações gerados pela expressão anterior, que era bem mais simples, conseguiram resolver este caso. Então o algoritmo de Remez pôde ser utilizado com incógnitas adicionais correspondentes às potências, o que diminuiu muito o trabalho.

A expressão resultante passou a ter um erro máximo da ordem de 0,05\% para a Eq. (1.5), o qual é muito menor do que os que foram obtidos até o momento. Além disso, ocorreu uma coincidência favorável a uma simplificação desta expressão: $q=0,50437$, o que nos leva a utilizar 
$q=0,5$, ou seja, é possível se utilizar a potência fracionária mais simples, a raiz quadrada, sem perda significativa de exatidão.

Apesar da obtenção da solução dos sistemas de equações, a busca dos valores finais ainda foi árdua, porque os sistemas levavam a várias soluções, que eram reordenadas de maneira diferente a cada iteração, e não havia como se distinguir entre algumas delas qual seria a correta, sendo necessário se testar mais de uma delas nestes casos.

Tudo isso foi feito para as três equações, e o resultado final foi compensador por se tratar de uma expressão simples e de grande exatidão:

$$
\beta_{1} \cong \frac{\sqrt{m A^{2} c+\beta_{1, \infty}^{2} c^{2 k}}}{A+c^{k}}
$$

onde $k=1,037$ e $A=4,025$ para a Eq. (1.5), $k=1,062$ e $A=5,63$ para a Eq. (1.6), e $k=1,081$ e $A=$ 7,25 para a Eq. (1.7). Novamente estes resultados foram confirmados pelas iterações gráficas e esta expressão está identificada como "Algébrica" na Figura 8.13, Figura 8.14 e Figura 8.15, respectivamente. 


\section{NOVAS EXPRESSÕES APROXIMADAS PARA A PRIMEIRA RAIZ OBTIDAS A PARTIR DA APROXIMAÇÃO DE PADÉ}

A aproximação de Padé foi utilizada para se obter uma nova família de funções iterativas no capítulo 3, e agora será utilizada para obter expressões aproximadas das raízes das Eqs. (1.5), (1.6) e (1.7).

A aproximação de Padé é uma função racional que, de modo muito análogo ao polinômio de Taylor, pode ser utilizada para aproximar uma função. A expansão em frações contínuas também pode ser considerada como uma função racional, mas é escrita de um modo diferente:

$$
\frac{a_{1}(x)}{b_{1}(x)+\frac{a_{2}(x)}{b_{2}(x)+\frac{a_{3}(x)}{b_{3}(x)+\cdots}}}
$$

onde $a_{n}$ e $b_{n}$ são polinômios em $x$ (LORENTZEN; 2010). Há forte relação entre estas três aproximações e, nos casos que serão apresentados, a expansão em frações contínuas também será caso particular da aproximação de Padé.

\subsection{Expressões Geradas por Sistemas Algébricos Computacionais}

A aproximação MiniMax está disponível no MatLab ${ }^{\circledR}$ 2015a apenas para problemas de processamento de sinais. 0 Maple ${ }^{\circledR} 2015$ e o Wolfram Mathematica ${ }^{\circledR} 10$ disponibilizam a aproximação MiniMax para funções racionais, sendo que por este último é possível se encontrar a aproximação de funções definidas parametricamente.

As Eqs. (1.5), (1.6) e (1.7) podem facilmente passar da forma implícita para a forma paramétrica. Por exemplo, a Eq. (1.5) pode ser reescrita como:

$$
\left\{\begin{array}{l}
\beta=t \\
c=t \tan t
\end{array}\right.
$$

que é implementada no Wolfram Mathematica ${ }^{\circledR} 10$.

É necessário se especificar o intervalo para que a expressão racional MiniMax seja determinada, além dos graus do numerador e do denominador. Após várias simulações, o melhor resultado ocorreu com o intervalo $[0,01,100]$ e com a ordem $[3,2]$ :

$$
\beta_{1}=\frac{0,0496+6,892 c+26,04 c^{2}+0,0055 c^{3}}{1+20,32 c+16,82 c^{2}}
$$


onde os valores dos parâmetros foram arredondados um a um, até um máximo que não acarretasse em diferença perceptível no resultado. Esta expressão tem erro relativo máximo de $0,7 \%$ no intervalo escolhido, conforme Figura 6.1, mas que cresceu proibitivamente fora desse intervalo, devido a essa expressão não ter valores corretos quando $c$ tende a zero ou infinito.

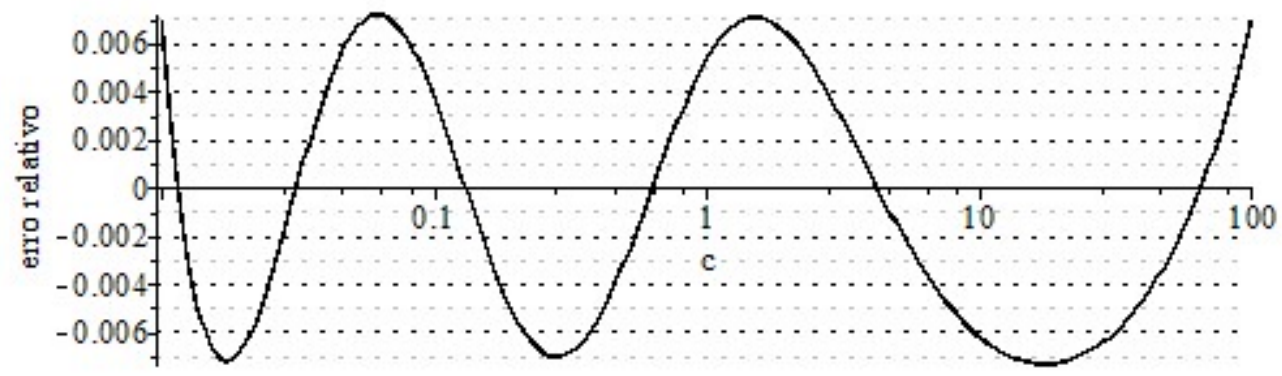

Figura 6.1 Erro relativo das raízes da Eq. (1.5) calculadas pela primeira forma da expressão racional gerada pelos sistemas algébricos computacionais

Em vez de se aproximar diretamente as soluções por expressões racionais MiniMax, uma outra iniciativa foi a de se aproximar o lado esquerdo das equações por estas expressões e então resolver as equações resultantes.

Aplicaremos essa ideia à Eq. (1.5) utilizando a máxima ordem que permite solução por equação quadrática, que é a ordem [2,2]. Naturalmente, o Maple ${ }^{\circledR} 2015$ e o Wolfram Mathematica ${ }^{\circledR}$ 10 levaram aos mesmos resultados. Observou-se que ao expandir o intervalo para um dos lados, a aproximação se tornava melhor para este lado, porém, a expansão dos dois lados simultaneamente levava a divergência. Procurou-se, então, o equilíbrio entre ambos lados através de simulações, o qual foi encontrado com o intervalo $c \in[0,031618,1,47292]$ que corresponde ao intervalo das raízes $\beta \in$ $[0,001,15]$.

Assim, para este intervalo, a aproximação MiniMax é:

$$
\beta \tan \beta=\frac{0,00030275-0,013189 \beta+1,4995 \beta^{2}}{1,3552+0,40372 \beta-0,79806 \beta^{2}}
$$

que leva a:

$$
\frac{0,00030275-0,013189 \beta+1,4995 \beta^{2}}{1,3552+0,40372 \beta-0,79806 \beta^{2}}=c
$$

de onde se obtém:

$$
\beta_{1}=\frac{0,253 c+0,008263+\sqrt{1,7 c^{2}+3,194 c-0,000644}}{c+1,879}
$$


que já apresenta um resultado um pouco melhor, porque, apesar do erro relativo máximo ser o mesmo, o intervalo para $c$ é mais amplo, conforme pode ser visto na Figura 6.2.

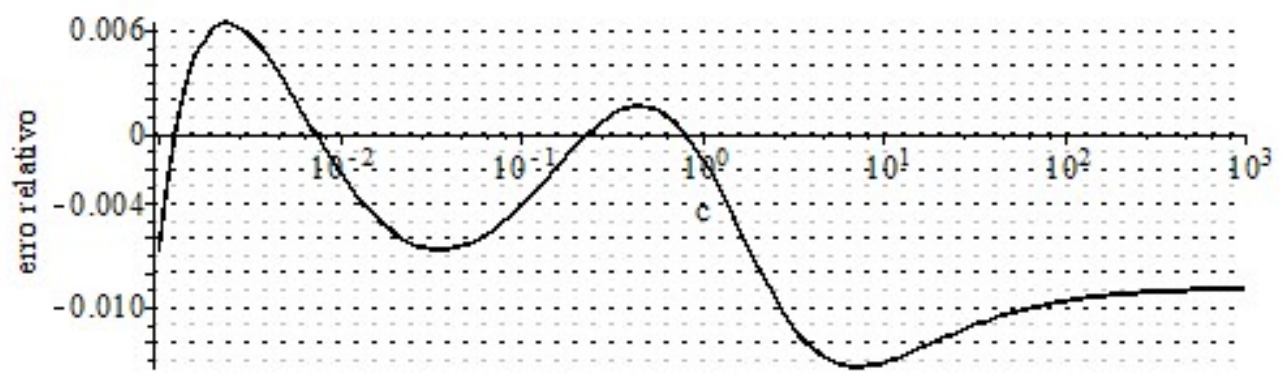

Figura 6.2 Erro relativo das raízes da Eq. (1.5) calculadas pela expressão algébrica obtida indiretamente da expressão racional pelos sistemas algébricos computacionais

Deixa-se registrado aqui que através da substituição de variável:

$$
\beta=\arctan \zeta
$$

onde $0 \leq \beta \leq \pi / 2$ e $0 \leq \zeta<\infty$, a Eq. (1.5) pode ser reescrita como:

$$
\zeta \arctan \zeta=c
$$

que tem um lado esquerdo que não é mais uma função com assíntota vertical. Porém, ela não leva a melhor resultado.

Será visto na próxima seção que as funções que aparecem do lado esquerdo das Eqs. (1.5), (1.6) e (1.7) podem ser escritas em termos de potências pares de $\beta$. Os sistemas algébricos computacionais não puderam ser utilizados em várias oportunidades ao longo deste trabalho, levando-nos a executar o algoritmo de Remes manualmente, justamente por eles servirem apenas para expressões racionais com todas as potências.

Entretanto, uma modificação nas equações permite a utilização desses sistemas para obter expressões apenas com potências pares. Através da mudança de variável:

$$
\zeta=\beta^{2}
$$

a Eq. (1.5) pode ser reescrita como:

$$
\sqrt{\zeta} \tan \sqrt{\zeta}=c
$$

onde o lado esquerdo pode ser aproximado por expressões racionais MiniMax em várias ordens.

Utilizando ordem [1/1], obtém-se:

$$
\sqrt{\zeta} \tan \sqrt{\zeta}=\frac{0,00001385+0,9645 \zeta}{1-0,392 \zeta}
$$

que leva a: 


$$
\frac{0,00001385+0,9645 \beta^{2}}{1-0,392 \beta^{2}}=c
$$

e, portanto:

$$
\beta_{1} \cong \sqrt{\frac{2,55 c}{c+2,46}}
$$

Apesar desta expressão não ter as tendências corretas, ela tem um erro que não aumenta ilimitadamente para os extremos do intervalo, conforme pode ser visto na Figura 6.3.

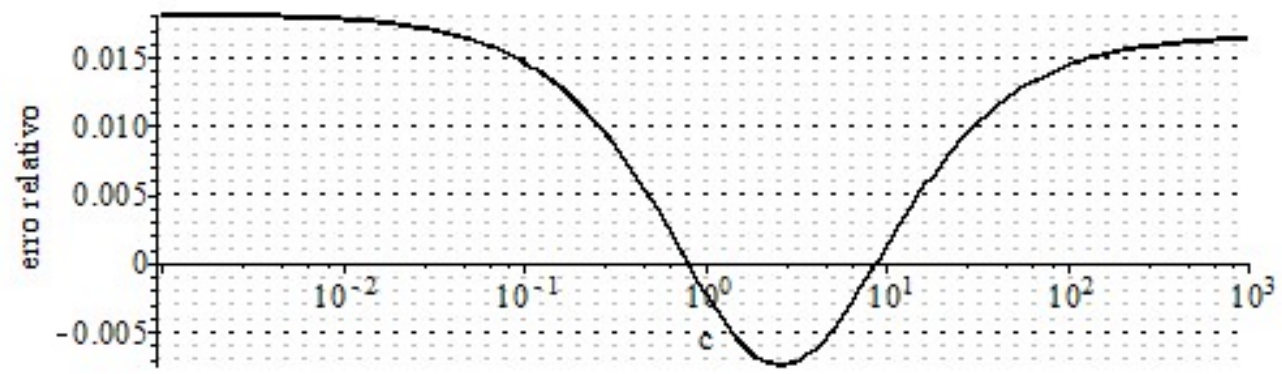

Figura 6.3 Erro relativo das raízes da Eq. (1.5) calculadas pela expressão algébrica obtida indiretamente da expressão racional de ordem [1/1] gerada pelos sistemas algébricos computacionais

Utilizando ordem [1/2], analogamente se obtém:

$$
\beta_{1} \cong \sqrt{\frac{\sqrt{0,2364 c^{2}+0,64 c+1}-1-0,32 c}{0,06718 c}}
$$

onde se observa que se o valor de $c$ for muito baixo em relação ao número de dígitos que utiliza a máquina que estiver efetuando o cálculo, então $\sqrt{0,2364 c^{2}+0,64 c+1} \cong 1$, o que adicionará erro devido a truncamento de dígitos.

Este erro é muito conhecido em cálculo numérico, e a fórmula quadrática (conhecida no Brasil como fórmula de Báskara) pode ser modificada para que ele não ocorra:

$$
a x^{2}+b x+c=0 \rightarrow x=\frac{-b \pm \sqrt{b^{2}-4 a c}}{2 a}=\frac{-2 c}{b \pm \sqrt{b^{2}-4 a c}}
$$

onde se observa que o valor baixo de $c$ não causa erro em pelo menos uma das raízes de cada expressão, conforme $b$ for positivo ou negativo.

Esta igualdade de fórmulas pode ser provada por multiplicações por conjugados e, da mesma maneira, pode-se modificar a expressão obtida para se chegar a uma forma que não tem essa restrição numérica: 


$$
\beta_{1} \cong \sqrt{\frac{6,227 c}{c+3,13+\sqrt{2,3 c^{2}+6,3 c+9,7}}}
$$

Esta expressão (em qualquer uma das duas formas acima) tem erro máximo bem menor do que o da expressão obtida a partir da ordem $[1,1]$, como pode ser visto na Figura 6.4

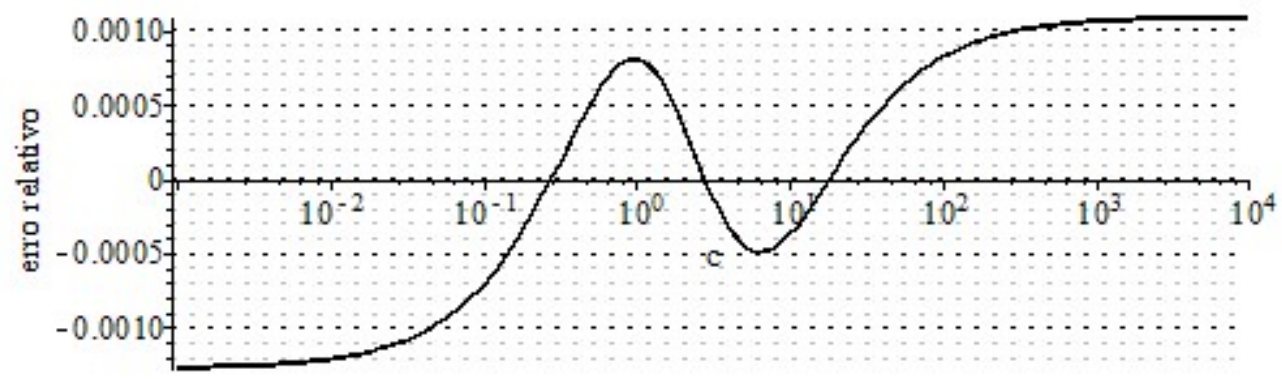

Figura 6.4 Erro relativo das raízes da Eq. (1.5) calculadas pela expressão algébrica obtida indiretamente da expressão racional de ordem [1/2] gerada pelos sistemas algébricos computacionais

Utilizando ordem [2/1], analogamente se obtém:

$$
\beta_{1} \cong \sqrt{2,713 c+6,704-\sqrt{(2,713 c+6,704)^{2}-13,398 c}}
$$

Apesar da ordem [2/1] ser equivalente à ordem [1/2], esta expressão tem erro máximo muito menor, como pode ser visto na Figura 6.5. Durante o desenvolvimento de expressões iterativas, observou-se em várias oportunidades que o denominador era mais importante para a convergência do que o numerador, mas agora observou-se esta inversão.

Esta expressão é a que tem menor erro de todas desenvolvidas até aqui. Porém, com uma pequena mudança em seus valores, ela levará a resultados melhores, o que será feito mais adiante.

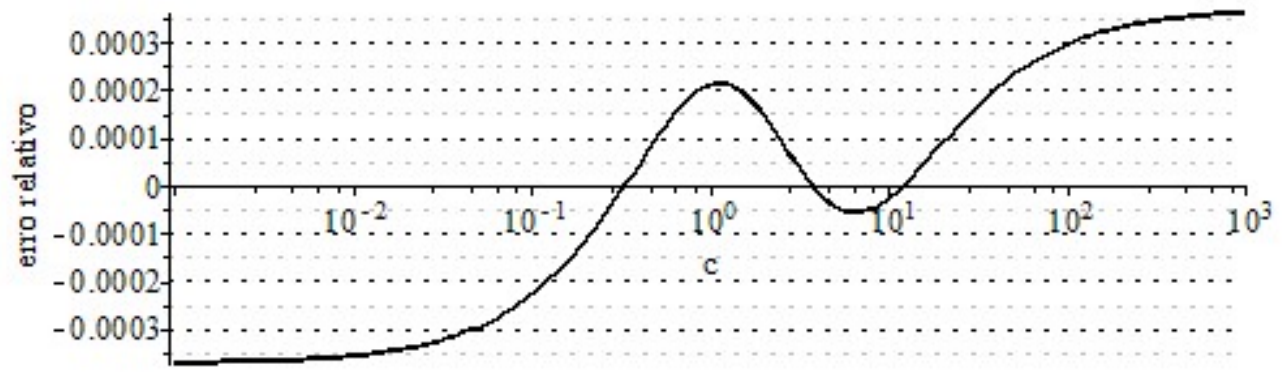

Figura 6.5 Erro relativo das raízes da Eq. (1.5) calculadas pela expressão algébrica obtida indiretamente da expressão racional de ordem [2/1] gerada pelos sistemas algébricos computacionais

Finalmente, utilizando ordem [2/2], se obtém: 


$$
\beta_{1}=\sqrt{\frac{c+2,32074-\sqrt{0,776384 c^{2}+2,541112 c+2,32074^{2}}}{0,04817737 c+0,452517}}
$$

a qual também tem uma subtração que poderá adicionar erro a seu resultado. Utilizando de multiplicações por conjugados, e arredondando-se novamente os parâmetros, obtém-se:

$$
\beta_{1}=\sqrt{\frac{(381 c+3578,6) c}{(c+9,3927)\left(82,086 c+190,5+\sqrt{5231,2 c^{2}+17122 c+36288}\right.}}
$$

que tem erro máximo extremamente menor, como pode ser visto na Figura 6.6, e que tem todos esses dígitos significativos necessários, devido a seu grau de exatidão.

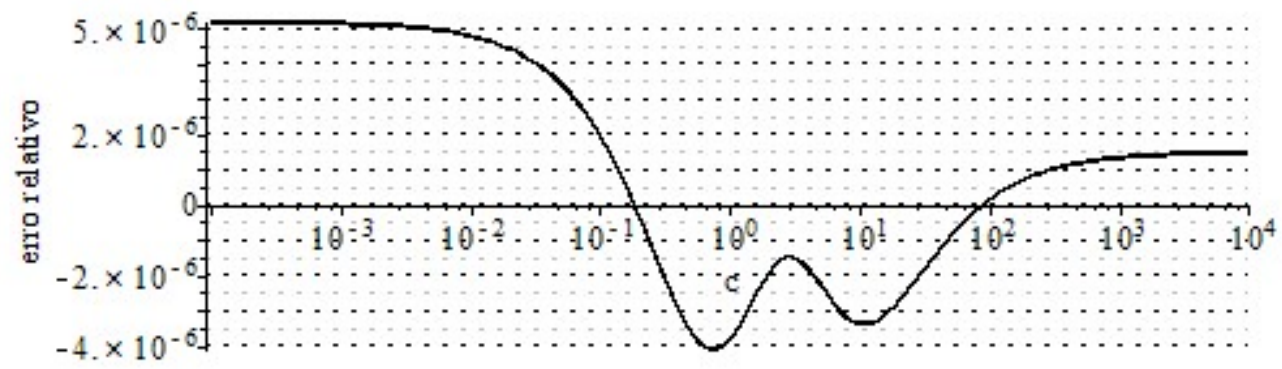

Figura 6.6 Erro relativo das raízes da Eq. (1.5) calculadas pela expressão algébrica obtida indiretamente da expressão racional de ordem [2/2] gerada pelos sistemas algébricos computacionais

Estas expressões não foram obtidas com tanta facilidade como podem aparentar. Os sistemas não conseguiam encontrar a MiniMax para intervalos grandes de $c$, então foi necessário se procurar os intervalos que levassem aos melhores resultados, através de exaustivo processo de tentativa e erro. A diminuição de um dos extremos do intervalo só podia ser conseguida com o aumento do outro extremo, então sempre se procurou um equilíbrio entre os dois.

Apesar da exatidão destas expressões, ainda não será utilizada esta metodologia para as duas outras equações, porque primeiro incluiremos nelas as tendências corretas, o que diminuirá o erro, eliminará as divergências que ocorrem com o aumento dos intervalos, e também elucidará algumas coincidências observadas como, por exemplo, na última expressão, onde se encontrou acidentalmente que $5,38583 \cong 2,32074^{2}$.

Isto será conseguido substituindo esta abordagem com sistemas algébricos computacionais por uma abordagem analítica, e apenas os valores dos parâmetros que aparecem nestas expressões serão modificados: a forma será mantida a mesma e os resultados serão melhores. 


\subsection{A Expansão em Frações Contínuas e a Aproximação de Padé}

A expansão em frações contínuas das funções que estão presentes nas Eqs. (1.5), (1.6) e (1.7) são:

$$
\begin{aligned}
& \tan \beta=\frac{\beta}{1+\frac{\beta^{2}}{-3+\frac{\beta^{2}}{5+\frac{\beta^{2}}{-7+\frac{\beta^{2}}{9+\cdots}}}}} \\
& \frac{\mathrm{J}_{1}(\beta)}{\mathrm{J}_{0}(\beta)}=\frac{\beta}{2-\frac{\beta^{2}}{4-\frac{\beta^{2}}{6-\frac{\beta^{2}}{8-\frac{\beta^{2}}{10+\cdots}}}}} \\
& \cot \beta=\frac{1}{\beta}-\frac{\beta}{3-\frac{\beta^{2}}{5-\frac{\beta^{2}}{7-\frac{\beta^{2}}{9-\frac{\beta^{2}}{11-\cdots}}}}}
\end{aligned}
$$

É muito interessante se observar a convergência destas expressões. A Figura 6.7 exemplifica o caso da fração contínua truncada com os cinco quocientes parciais mostrados acima para a Eq. (1.6).

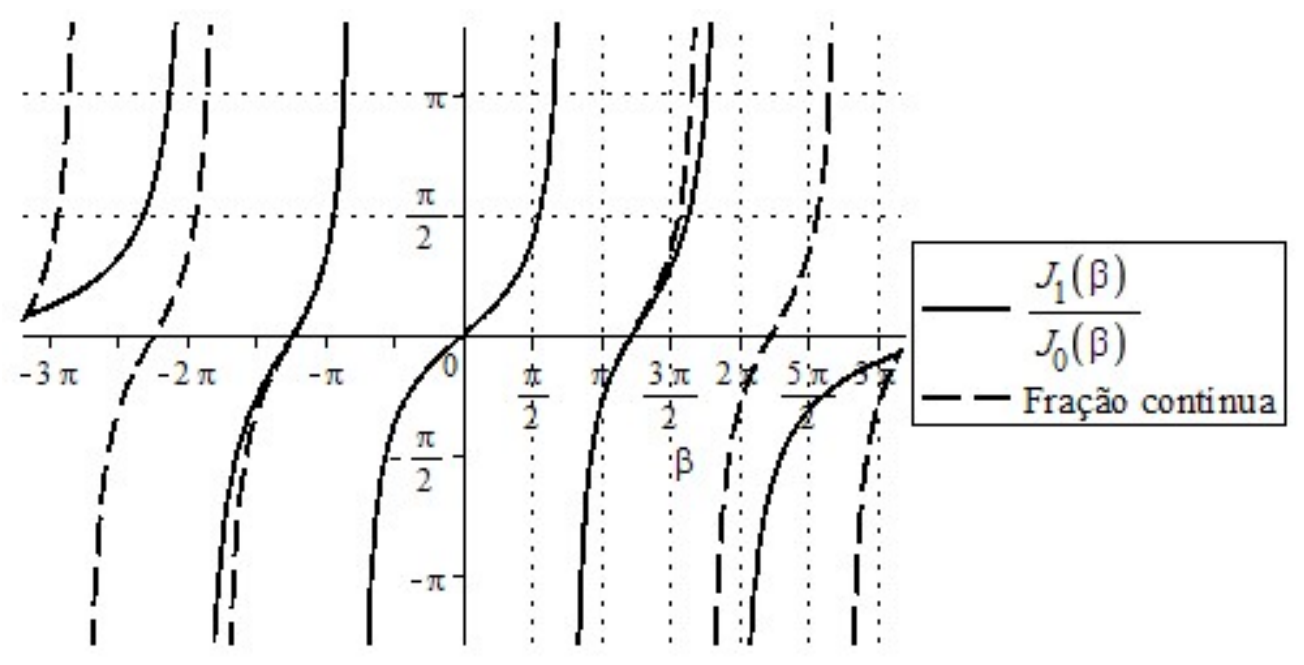

Figura 6.7 Aproximação da razão entre as funções de Bessel por fração contínua

As duas outras frações contínuas levam a gráficos análogos. Observe-se que até mesmo os pontos com assíntotas verticais podem ser aproximados através das raízes dos denominadores das 
funções racionais. Quanto maior o grau do denominador, maior o número de raízes, e mais assíntotas podem ser representadas.

Substituindo na Eq. (1.5) a respectiva fração contínua, obtém-se:

$$
\frac{\beta^{2}}{1+\frac{\beta^{2}}{-3+\frac{\beta^{2}}{5+\frac{\beta^{2}}{-7+\frac{\beta^{2}}{9+\cdots}}}}}=c
$$

Substituindo na Eq. (1.6), obtém-se:

$$
\frac{\beta^{2}}{2-\frac{\beta^{2}}{4-\frac{\beta^{2}}{6-\frac{\beta^{2}}{8-\frac{\beta^{2}}{10+\cdots}}}}}=c
$$

E na Eq. (1.7), obtém-se:

$$
\frac{\beta^{2}}{3-\frac{\beta^{2}}{5-\frac{\beta^{2}}{7-\frac{\beta^{2}}{9-\frac{\beta^{2}}{11-\cdots}}}}}=c
$$

A equivalência entre as três equações sob esta forma é notável, e mais uma vez se confirma a melhor opção de se utilizar $(1-c)$ em vez de simplesmente $c$ na Eq. (1.7).

0 truncamento com apenas um termo da Eq. (6.5) leva a:

$$
\frac{\beta^{2}}{1}=c \rightarrow \beta_{1}=\sqrt{c}
$$

a da Eq. (6.6) leva a:

$$
\frac{\beta^{2}}{2}=c \rightarrow \beta_{1}=\sqrt{2 c}
$$

e a da Eq. (6.7) leva a:

$$
\frac{\beta^{2}}{3}=c \rightarrow \beta_{1}=\sqrt{3 c}
$$

Ou seja, a Eq. (2.12) é obtida através do truncamento para apenas um quociente parcial.

Consideremos incialmente a Eq. (6.5). Ela pode ser resolvida por equação quadrática com as seguintes quantidades de quocientes: 


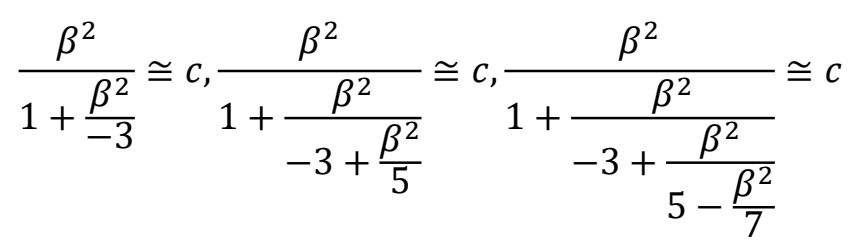

Este conjunto de equações pertence a um conjunto maior que é obtido através da aproximação de Padé de várias ordens da função $\beta \tan \beta$ :

$$
\begin{gathered}
\beta \tan \beta \cong \frac{\beta^{2}}{1-\frac{1}{3} \beta^{2}} \\
\beta \tan \beta \cong \frac{\beta^{2}}{1-\frac{1}{3} \beta^{2}-\frac{1}{45} \beta^{4}} \\
\beta \tan \beta \cong \frac{\beta^{2}-\frac{1}{15} \beta^{4}}{1-\frac{2}{5} \beta^{2}} \\
\beta \tan \beta \cong \frac{\beta^{2}-\frac{2}{21} \beta^{4}}{1-\frac{3}{7} \beta^{2}+\frac{1}{105} \beta^{4}}
\end{gathered}
$$

as quais levam, respectivamente, a:

$$
\begin{gathered}
\beta_{1} \cong \sqrt{\frac{3 c}{c+3}} \\
\beta_{1} \cong \sqrt{\frac{3}{2 c}\left(\sqrt{45 c^{2}+150 c+225}-5(c+3)\right)} \\
\beta_{1} \cong \sqrt{3 c+7,5-\sqrt{(3 c+7,5)^{2}-15 c}} \\
\beta_{1} \cong \sqrt{\frac{15(3 c+7)-\sqrt{15\left(107 c^{2}+350 c+735\right)}}{2(c+10)}}
\end{gathered}
$$

Estas expressões resultantes possuem valores máximos de erro, respectivamente, de 10\%, 2\%, 0,65\% e 0,03\%. Repetindo-se esse procedimento a partir da Eq. (6.6), obtém-se:

$$
\begin{gathered}
\beta_{1} \cong \sqrt{\frac{8 c}{c+4}} \\
\beta_{1} \cong \sqrt{\frac{4}{c}\left(\sqrt{21 c^{2}+72 c+144}-3(c+4)\right)} \\
\beta_{1} \cong \sqrt{c+3-\sqrt{(c+3)^{2}-3 c}}
\end{gathered}
$$




$$
\beta_{1} \cong \sqrt{\frac{12(3 c+8)-4 \sqrt{3\left(19 c^{2}+48 c+192\right)}}{c+12}}
$$

as quais possuem erros máximos, respectivamente, de 16\%, 4,5\%, 2\% e 0,15\%. Finalmente, a partir da Eq. (6.7), obtém-se

$$
\begin{gathered}
\beta_{1} \cong \sqrt{\frac{15 c}{c+5}} \\
\beta_{1} \cong \sqrt{\frac{5}{2 c}\left(\sqrt{133 c^{2}+490 c+1225}-7(c+5)\right)} \\
\beta_{1} \cong \sqrt{5 c+17,5-\sqrt{25 c^{2}+70 c+306,25}} \\
\beta_{1} \cong \sqrt{\frac{\left.105(c+3)-3 \sqrt{35\left(23 c^{2}+42 c+315\right.}\right)}{2(c+14)}}
\end{gathered}
$$

as quais possuem erros máximos, respectivamente, de $23 \%, 7 \%, 3 \%$ e 0,35\%. Apenas as últimas equações correspondem a expressões que tem exatidões que sejam comparáveis às expressões já desenvolvidas. Na Figura 8.13, Figura 8.14 e Figura 8.15, respectivamente, as expressões das Eqs. (6.8), (6.9) e (6.10) estão identificadas como "Frações Parciais".

Observa-se que estas expressões têm apenas diferenças nos valores dos parâmetros em relação as das Eqs. (6.1) a (6.4), mas exatamente as mesmas formas. Então, nosso objetivo agora é o de descobrir os valores desses parâmetros que levem essas expressões aos melhores resultados.

\subsection{Uma Expressão Obtida a Partir da Aproximação de Padé de Ordem $[1,1]$}

Iniciaremos pela primeira forma:

$$
\beta_{1} \cong \sqrt{\frac{x_{1} c}{x_{2} c+1}}
$$

Para que ela tenha valor correto quando $c$ tende a zero, é necessário que:

$$
\lim _{c \rightarrow 0^{+}} \sqrt{\frac{x_{1} c}{x_{2} c+1}}=0
$$

o que é automaticamente satisfeito para quaisquer valores de parâmetros $x_{1}$ e $x_{2}$.

Para que esta expressão tenha a tendência da Eq. (2.12), é necessário que 


$$
\lim _{c \rightarrow 0^{+}} \frac{\sqrt{\frac{x_{1} c}{x_{2} c+1}}}{\sqrt{m c}}=\frac{x_{1}}{m}=1
$$

Para que ela tenha valor correto quando $c$ tende a infinito, é necessário que:

$$
\lim _{c \rightarrow \infty} \sqrt{\frac{x_{1} c}{x_{2} c+1}}=\sqrt{\frac{x_{1}}{x_{2}}}=\beta_{1, \infty}
$$

Assim, resolvendo esse sistema de equações, a expressão é reescrita como:

$$
\beta_{1} \cong \sqrt{\frac{m c}{1+\frac{m c}{\beta_{1, \infty}^{2}}}}
$$

que já havia sido descoberta por Ostrogorsky e Mikic (2008), apresentada na Eq. (2.25), e utilizada no método do ponto fixo, levando as expressões das Eqs. (5.1) e (5.2).

\subsection{Uma Expressão Obtida a Partir da Aproximação de Padé de Ordem [1, 2]}

Consideremos a próxima forma:

$$
\beta_{1} \cong \sqrt{\frac{\sqrt{x_{1} c^{2}+x_{2} c+1}-1-x_{3} c}{x_{4} c}}
$$

que não foi a que deu melhores resultados, porém, é necessário verificar se a razão não é a de que os parâmetros estavam muito diferentes dos ideais.

Para que ela tenha valor correto quando $c$ tende a zero, é necessário que:

$$
\lim _{c \rightarrow 0^{+}} \sqrt{\frac{\sqrt{x_{1} c^{2}+x_{2} c+1}-1-x_{3} c}{x_{4} c}}=\sqrt{\frac{x_{2}-2 x_{3}}{2 x_{4}}}=0
$$

Para que esta expressão tenha a tendência da Eq. (2.12), é necessário que

$$
\lim _{c \rightarrow 0^{+}} \frac{\sqrt{\frac{\sqrt{x_{1} c^{2}+x_{2} c+1}-1-x_{3} c}{x_{4} c}}}{\sqrt{m c}}=\sqrt{\frac{x_{1}-x_{3}^{2}}{2 m x_{4}}}=1
$$

Para que ela tenha valor correto quando $c$ tende a infinito, é necessário que:

$$
\lim _{c \rightarrow \infty} \sqrt{\frac{\sqrt{x_{1} c^{2}+2 x_{3} c+1}-1-x_{3} c}{x_{4} c}}=\sqrt{\frac{\sqrt{x_{1}}-x_{3}}{x_{4}}}=\beta_{1, \infty}
$$

Então, para que a expressão tenha as tendências corretas, ela deve ter a forma: 


$$
\beta_{1} \cong \sqrt{\frac{\sqrt{\left(x_{3}^{2}-\frac{4 m x_{3}}{\beta_{1, \infty}^{2}}+\frac{4 m^{2}}{\beta_{1, \infty}^{4}}\right) c^{2}+2 x_{3} c+1}-1-x_{3} c}{\left(\frac{2 m}{\beta_{1, \infty}^{4}}-\frac{2 x_{3}}{\beta_{1, \infty}^{2}}\right) c}}
$$

onde o valor de $x_{3}$ deve ser determinado para que o erro máximo tenha o menor valor possível.

Observe-se que a expressão da Eq. (6.2) não satisfaz exatamente essas condições:

$$
\begin{gathered}
x_{1}=x_{3}^{2}-\frac{4 m x_{3}}{\beta_{1, \infty}^{2}}+\frac{4 m^{2}}{\beta_{1, \infty}^{4}}=0,32^{2}-\frac{4 \cdot 1 \cdot 0,32}{\frac{\pi^{2}}{4}}+\frac{4 \cdot 1^{2}}{\frac{\pi^{4}}{16}}=0,2407 \neq 0,2364=x_{1} \\
\frac{2 \cdot 1}{\frac{\pi^{4}}{16}}-\frac{2 \cdot 0,32}{\frac{\pi^{2}}{4}}=0,06913 \neq 0,06718=x_{4}
\end{gathered}
$$

e consequentemente pode-se ver na Figura 6.4 que o erro relativo não se aproximam de zero nos extremos. A expressão equivalente obtida por Padé de Ordem [1,2] sem alteração dos parâmetros para o caso da Eq. (6.5) também não satisfaz exatamente essas condições:

$$
\begin{gathered}
x_{3}^{2}-\frac{4 m x_{3}}{\beta_{1, \infty}^{2}}+\frac{4 m^{2}}{\beta_{1, \infty}^{4}}=\left(\frac{1}{3}\right)^{2}-\frac{4 \cdot 1 \cdot \frac{1}{3}}{\frac{\pi^{2}}{4}}+\frac{4 \cdot 1^{2}}{\frac{\pi^{4}}{16}}=0,2281 \neq 0,2=x_{1} \\
\frac{2 \cdot 1}{\frac{\pi^{4}}{16}}-\frac{2 \cdot 0,333}{\frac{\pi^{2}}{4}}=0,0586 \neq 0,0444=x_{4}
\end{gathered}
$$

Por se tratar de um único parâmetro, é preferível utilizar diretamente as iterações gráficas em vez de se iniciar através do algoritmo de Remez, para então se confirmar os resultados através das iterações gráficas. Os resultados são $x_{3}=0,323,0,227$ e 0,167 que levam a erros de 0,13\%, 0,35\% e 0,5\%, para as Eqs. (1.5), (1.6) e (1.7), respectivamente.

Os resultados dessa expressão realmente não melhoraram suficientemente para que seja considerada nos gráficos comparativos. É interessante notar que justamente essa expressão não é um caso das frações contínuas.

\subsection{Uma Expressão Obtida a Partir da Aproximação de Padé de Ordem $[2,1]$}

Consideremos agora a forma:

$$
\beta_{1} \cong \sqrt{x_{1} c+x_{2}-\sqrt{\left(x_{3} c+x_{4}\right)^{2}-x_{5} c}}
$$

Para que ela tenha valor correto quando $c$ tende a zero, é necessário que: 


$$
\lim _{c \rightarrow 0^{+}} \sqrt{x_{1} c+x_{2}-\sqrt{\left(x_{3} c+x_{4}\right)^{2}-x_{5} c}}=0
$$

Para que ela tenha valor correto quando $c$ tende a infinito, é necessário que:

$$
\lim _{c \rightarrow \infty} \sqrt{x_{1} c+x_{2}-\sqrt{\left(x_{3} c+x_{2}\right)^{2}-x_{5} c}}=\sqrt{\frac{x_{5}}{2 x_{1}}}=\beta_{1, \infty}
$$

Para que tenha a tendência da Eq. (2.12), é necessário que

$$
\lim _{c \rightarrow 0^{+}} \frac{\sqrt{x_{1} c+x_{2}-\sqrt{\left(x_{1} c+x_{2}\right)^{2}-2 x_{1} \beta_{1, \infty}^{2} c}}}{\sqrt{m c}}=\sqrt{\frac{x_{1} \beta_{1, \infty}^{2}}{m x_{2}}}=1
$$

Portanto, a expressão com as tendências corretas é:

$$
\beta_{1} \cong \sqrt{x_{1} c+\frac{x_{1} \beta_{1, \infty}^{2}}{m}-\sqrt{\left(x_{1} c+\frac{x_{1} \beta_{1, \infty}^{2}}{m}\right)^{2}-2 x_{1} \beta_{1, \infty}^{2} c}}
$$

Incorporando esses resultados na expressão, e ajustando seus valores por gráficos para ela ser uma MiniMax, obtém-se:

$$
\beta_{1} \cong a \sqrt{c+\frac{\beta_{1, \infty}^{2}}{m}-\sqrt{\left(c+\frac{\beta_{1, \infty}^{2}}{m}\right)^{2}-\frac{2 \beta_{1, \infty}^{2}}{a^{2}} c}}
$$

onde $a=1,6508,1,8483$ e 2,0215 para a primeira raiz das Eqs. (1.5), (1.6) e (1.7), respectivamente. Ela está identificada como "Padé de Ordem [2, 1]" na Figura 8.13, Figura 8.14 e Figura 8.15, respectivamente.

\subsection{Uma Expressão Obtida a Partir da Aproximação de Padé de Ordem [2, 2]}

Consideremos agora a última forma:

$$
\beta_{1} \cong \sqrt{\frac{c+x_{1}-\sqrt{x_{4} c^{2}+x_{5} c+x_{6}}}{x_{2} c+x_{3}}}
$$

Para que ela tenha valor correto quando $c$ tende a zero, é necessário que:

$$
\lim _{c \rightarrow 0^{+}} \sqrt{\frac{c+x_{1}-\sqrt{x_{4} c^{2}+x_{5} c+x_{6}}}{x_{2} c+x_{3}}}=0
$$

e, então:

$$
x_{1}-\sqrt{x_{6}}=0
$$


o que explica a coincidência citada inicialmente.

Para que esta expressão tenha a tendência da Eq. (2.12), é necessário que

$$
\lim _{c \rightarrow 0^{+}} \frac{\sqrt{\frac{c+x_{1}-\sqrt{x_{4} c^{2}+x_{5} c+x_{1}^{2}}}{x_{2} c+x_{3}}}}{\sqrt{m c}}=\sqrt{\frac{2 x_{1}-x_{5}}{2 m x_{1} x_{3}}}=1
$$

Para que ela tenha valor correto quando $c$ tende a infinito, é necessário que:

$$
\lim _{c \rightarrow \infty} \sqrt{\frac{c+x_{1}-\sqrt{x_{4} c^{2}+x_{5} c+x_{1}^{2}}}{x_{2} c+x_{3}}}=\beta_{1, \infty}
$$

Assim, a expressão pode ser reescrita como:

$$
\beta_{1} \cong \sqrt{\frac{c+x_{1}-\sqrt{\left(1-\beta_{1, \infty}^{2} x_{2}\right)^{2} c^{2}+2 x_{1}\left(1-m x_{3}\right) c+x_{1}^{2}}}{x_{2} c+x_{3}}}
$$

a qual tem os três parâmetros $x_{1}, x_{2}$ e $x_{3}$ que podem ser determinados para melhor ajuste.

Então, utilizando gráficos, não foi muito difícil se obter os parâmetros para o caso da Eq. (1.5), porém o mesmo não pode ser dito em relação às demais equações e felizmente o algoritmo de Remez convergiu inclusive para confirmar os parâmetros obtidos para a primeira equação. Os resultados estão na Tabela 6.1, Tabela 6.2 e Tabela 6.3 para as Eqs. (1.5), (1.6) e (1.7), respectivamente.

Observou-se uma velocidade inicial de convergência muito alta. No primeiro cálculo do sistema não linear, o qual resulta nos dados da primeira linha dessas tabelas, os valores dos parâmetros já se aproximaram muito dos valores ótimos, e o erro máximo já se iniciou muito baixo (mostrado na última coluna das tabelas).

O tempo computacional é quase todo utilizado para se calcular o ponto de maior erro (mostrado na coluna do $c$ ). Então, optou-se por 'varrer' o intervalo iniciando do extremo esquerdo 0,01 e multiplicando sucessivamente este valor por 1,001 até chegar a 100. Após isso, passou-se a utilizar valores menores como 1,0001 e 1,00001. Não há qualquer preocupação com divergência devido a esse procedimento porque a estimativa inicial $[0,1,1,10,100]$ é absolutamente arbitrária, e qualquer linha pode ser considerada como um novo reinício.

Assim, o menor erro possível foi encontrado com valores de parâmetros que podem ser arredondados para $x_{1}=2,3250266, x_{2}=0,0468978$ e $x_{3}=0,449981$ para a Eq. (1.5), $x_{1}=$ 2,636312, $x_{2}=0,030765$ e $x_{3}=0,340892$ para a Eq. (1.6), e $x_{1}=2,937165, x_{2}=0,0220076$ e $x_{3}=$ 0,274973 para a Eq. (1.7), e os resultados estão na Figura 8.17. 
Tabela 6.1 Resultados do algoritmo de troca de Remez para a primeira raiz da Eq. (1.5) calculada pela Eq. (6.12)

\begin{tabular}{|c|c|c|c|c|c|c|}
\hline Abscissas & $x_{1}$ & $x_{2}$ & $x_{3}$ & $h$ & $c$ & $H$ \\
\hline $\left.\begin{array}{llllllllllll}0,1 & 1 & 10 & 1 & 0\end{array}\right]$ & 2,3249342 & 0,0469233 & 0,4500413 & $6,25 \cdot 10^{-7}$ & 1,666797658 & $1,24 \cdot 10^{-6}$ \\
\hline$\left[\begin{array}{llllll}0,1 & 1,667 & 10 & 100\end{array}\right]$ & 2,3250244 & 0,0468989 & 0,4499782 & $6,91 \cdot 10^{-7}$ & 6,144625098 & $1,19 \cdot 10^{-6}$ \\
\hline$[0,11,6676,145100]$ & 2,3248931 & 0,0469415 & 0,4500582 & $7,57 \cdot 10^{-7}$ & 0,3079470685 & $1,25 \cdot 10^{-6}$ \\
\hline$[0,30791,6676,145100]$ & 2,3252894 & 0,0468224 & 0,4498184 & $8,88 \cdot 10^{-7}$ & 35,76783086 & $1,34 \cdot 10^{-6}$ \\
\hline$[0,3079$ 1,667 6,145 35,77] & 2,3250260 & 0,0468980 & 0,4499812 & $9,86 \cdot 10^{-7}$ & 0,2727371545 & $9,98 \cdot 10^{-7}$ \\
\hline$[0,2727$ 1,667 6,145 35,77] & 2,3250308 & 0,0468966 & 0,4499784 & $9,89 \cdot 10^{-7}$ & 38,62418076 & $9,99 \cdot 10^{-7}$ \\
\hline$[0,27271,6676,14538,62]$ & 2,3250261 & 0,0468979 & 0,4499812 & $9,91 \cdot 10^{-7}$ & 0,2730782875 & $9,95 \cdot 10^{-7}$ \\
\hline$[0,2731$ 1,667 6,145 38,62] & 2,3250262 & 0,0468979 & 0,4499812 & $9,91 \cdot 10^{-7}$ & 0,2758558565 & $9,95 \cdot 10^{-7}$ \\
\hline$[0,27591,6676,14538,62]$ & 2,3250263 & 0,0468979 & 0,4499812 & $9,91 \cdot 10^{-7}$ & 0,2715071705 & $9,95 \cdot 10^{-7}$ \\
\hline$[0,27151,6676,14538,62]$ & 2,3250262 & 0,0468979 & 0,4499812 & $9,91 \cdot 10^{-7}$ & 0,2764357595 & $9,95 \cdot 10^{-7}$ \\
\hline$[0,2764$ 1,667 $6,14538,62]$ & 2,3250266 & 0,0468978 & 0,4499810 & $9,91 \cdot 10^{-7}$ & 0,2753184645 & $9,96 \cdot 10^{-7}$ \\
\hline$\left[\begin{array}{lllll}0,2753 & 1,667 & 6,145 & 38,62\end{array}\right]$ & 2,3250266 & 0,0468978 & 0,4499810 & $9,91 \cdot 10^{-7}$ & & \\
\hline
\end{tabular}

Tabela 6.2 Resultados do algoritmo de troca de Remez para a primeira raiz da Eq. (1.6) calculada pela Eq. (6.12)

\begin{tabular}{|c|c|c|c|c|c|c|}
\hline Abscissas & $x_{1}$ & $x_{2}$ & $x_{3}$ & $h$ & $c$ & $H$ \\
\hline$\left[\begin{array}{lllll}0,1 & 1 & 10 & 100\end{array}\right]$ & 2,6348589 & 0,03090463 & 0,3412321 & $3,02 \cdot 10^{-6}$ & 2,204630855 & $1,15 \cdot 10^{-5}$ \\
\hline$\left[\begin{array}{lllll}0,1 & 2,205 & 10 & 100\end{array}\right]$ & 2,6352128 & 0,03088273 & 0,3411090 & $3,93 \cdot 10^{-6}$ & 0,4154651714 & $8,10 \cdot 10^{-6}$ \\
\hline$\left[\begin{array}{llllll}0,4155 & 2,205 & 10 & 100\end{array}\right]$ & 2,6371223 & 0,03068176 & 0,3407186 & $4,81 \cdot 10^{-6}$ & 6,892287978 & $6,84 \cdot 10^{-6}$ \\
\hline$\left[\begin{array}{llllll}0,4155 & 2,205 & 6,892 & 100\end{array}\right]$ & 2,6369688 & 0,03070218 & 0,3407502 & $5,20 \cdot 10^{-6}$ & 40,50051052 & $7,23 \cdot 10^{-6}$ \\
\hline$[0,41552,2056,89240,50]$ & 2,6362798 & 0,03076805 & 0,3408989 & $5,64 \cdot 10^{-6}$ & 0,3539680992 & $5,75 \cdot 10^{-6}$ \\
\hline$[0,35402,2056,89240,50]$ & 2,6363244 & 0,03076335 & 0,3408898 & $5,67 \cdot 10^{-6}$ & 2,037906836 & $5,78 \cdot 10^{-6}$ \\
\hline$[0,35402,0386,89240,50]$ & 2,6363328 & 0,03076266 & 0,3408875 & $5,70 \cdot 10^{-6}$ & 44,70242703 & $5,75 \cdot 10^{-6}$ \\
\hline$[0,35402,0386,89244,70]$ & 2,6363184 & 0,03076403 & 0,3408906 & $5,71 \cdot 10^{-6}$ & 7,249821922 & $5,76 \cdot 10^{-6}$ \\
\hline$[0,35402,038 \quad 7,25044,70]$ & 2,6363121 & 0,03076476 & 0,3408920 & $5,72 \cdot 10^{-6}$ & 0,3517065805 & $5,72 \cdot 10^{-6}$ \\
\hline$[0,35172,0387,25044,70]$ & 2,6363117 & 0,03076480 & 0,3408921 & $5,72 \cdot 10^{-6}$ & & \\
\hline
\end{tabular}

Tabela 6.3 Resultados do algoritmo de troca de Remez para a primeira raiz da Eq. (1.7) calculada pela Eq. (6.12)

\begin{tabular}{ccccccc}
\hline Abscissas & $x_{1}$ & $x_{2}$ & $x_{3}$ & $h$ & $c$ & $H$ \\
\hline$[0,1110100]$ & 2,9331966 & 0,0221881 & 0,2754676 & $6,12 \cdot 10^{-6}$ & 2,623872756 & $3,80 \cdot 10^{-5}$ \\
{$[0,12,62410100]$} & 2,9332943 & 0,0222139 & 0,2753433 & $9,54 \cdot 10^{-6}$ & 0,5365104294 & $2,42 \cdot 10^{-5}$ \\
{$[0,53652,62410100]$} & 2,9382152 & 0,0219564 & 0,2748602 & $1,28 \cdot 10^{-5}$ & 47,09689287 & $1,64 \cdot 10^{-5}$ \\
{$[0,53652,6241047,10]$} & 2,9371352 & 0,0220076 & 0,2749759 & $1,38 \cdot 10^{-5}$ & 8,191478999 & $1,54 \cdot 10^{-5}$ \\
{$[0,53652,6248,19147,10]$} & 2,9369712 & 0,0220174 & 0,2749927 & $1,42 \cdot 10^{-5}$ & 0,4313832495 & $1,47 \cdot 10^{-5}$ \\
{$[0,43142,6248,19147,10]$} & 2,9371425 & 0,0220084 & 0,2749761 & $1,44 \cdot 10^{-5}$ & 2,379894729 & $1,48 \cdot 10^{-5}$ \\
{$[0,43142,3808,19147,10]$} & 2,9371702 & 0,0220073 & 0,2749720 & $1,45 \cdot 10^{-5}$ & 48,95234831 & $1,45 \cdot 10^{-5}$ \\
{$[0,43142,3808,19148,95]$} & 2,9371654 & 0,0220076 & 0,2749726 & $1,45 \cdot 10^{-5}$ & 0,4295191184 & $1,45 \cdot 10^{-5}$ \\
{$[0,42952,3808,19148,95]$} & 2,9371655 & 0,0220076 & 0,2749726 & $1,45 \cdot 10^{-5}$ & 8,136387854 & $1,45 \cdot 10^{-5}$ \\
{$[0,43142,3808,13648,95]$} & 2,9371654 & 0,0220076 & 0,2749726 & $1,45 \cdot 10^{-5}$ & & \\
\hline
\end{tabular}




\section{NOVAS EXPRESSÕES APROXIMADAS PARA AS DEMAIS RAÍZES}

\subsection{A Generalização da Expressão Obtida a Partir da Aproximação de Padé de Ordem [1,} 1]

Uma expressão para todas as raízes da Eq. (1.5) pode ser obtida a partir da aproximação de Padé de ordem $[1,1]$ da função tangente em torno do ponto $\beta=0$ que já foi utilizada para a primeira raiz:

$$
\tan \beta_{1} \cong \frac{\beta_{1}}{1-\frac{1}{3} \beta_{1}^{2}}
$$

Devido a periodicidade da função tangente, essa equação pode ser modificada para ser válida para qualquer raiz:

$$
\tan \beta_{n} \cong \frac{\beta_{n}-(n-1) \pi}{1-\frac{1}{3}\left(\beta_{n}-(n-1) \pi\right)^{2}}
$$

e, então, a Eq. (1.5) pode ser aproximada por:

$$
\beta_{n} \frac{\beta_{n}-(n-1) \pi}{1-\frac{1}{3}\left(\beta_{n}-(n-1) \pi\right)^{2}} \cong c
$$

que tem solução:

$$
\beta_{n} \cong \frac{\pi(n-1)(c+3 / 2)+\sqrt{3 c^{2}+9 c+9 / 4 \pi^{2}(n-1)^{2}}}{c+3}
$$

Como anteriormente, seus parâmetros podem ser substituídos por outros que levem a melhores resultados:

$$
\beta_{n} \cong \frac{x_{1}(n-1)\left(c+x_{2}\right)+\sqrt{x_{3} c^{2}+x_{4} c+x_{5}(n-1)^{2}}}{c+x_{6}}
$$

Para que esta expressão tenha valores corretos quando $c$ tende a zero, é necessário que

$$
\lim _{c \rightarrow 0^{+}} \frac{x_{1}(n-1)\left(c+x_{2}\right)+\sqrt{x_{3} c^{2}+x_{4} c+x_{5}(n-1)^{2}}}{c+x_{6}}=\frac{x_{1} x_{2}+\sqrt{x_{5}}}{x_{6}}(n-1)=(n-1) \pi
$$

Para que ela tenha valores corretos quando $c$ tende a infinito:

$$
\lim _{c \rightarrow \infty} \frac{x_{1}(n-1)\left(c+x_{2}\right)+\sqrt{x_{3} c^{2}+x_{4} c+x_{5}(n-1)^{2}}}{c+x_{6}}=n x_{1}+\sqrt{x_{3}}-x_{1}=\left(n-\frac{1}{2}\right) \pi
$$

e, devido a primeira raiz ser a mais importante, pode-se fazer com que ela satisfaça também a tendência da Eq. (2.12): 


$$
\lim _{c \rightarrow 0^{+}} \frac{1}{\sqrt{c}} \frac{x_{1}(1-1)\left(c+x_{2}\right)+\sqrt{x_{3} c^{2}+x_{4} c+x_{5}(1-1)^{2}}}{c+x_{6}}=\frac{\sqrt{x_{4}}}{x_{6}}=1
$$

Então, a expressão passa a ser:

$$
\beta_{n} \cong \frac{\pi(n-1)\left(c+x_{2}\right)+\sqrt{(\pi c / 2)^{2}+x_{6}^{2} c+\left(\pi\left(x_{6}-x_{2}\right)(n-1)\right)^{2}}}{c+x_{6}}
$$

onde os parâmetros $x_{2}$ e $x_{6}$ podem ser determinados para o melhor ajuste.

Novamente devido a primeira raiz ser a mais importante, o ajuste inicial será feito por ela. Substituindo $n$ por 1 nessa expressão, obtém-se:

$$
\beta_{1} \cong \frac{\sqrt{(\pi c / 2)^{2}+x_{6}^{2} c}}{c+x_{6}}
$$

que desacopla o parâmetro $x_{2}$ e permite que o ajuste de $x_{6}$ seja feito com o auxílio de gráficos. Através da Figura 7.1, observa-se que há dois valores possíveis para o parâmetro $x_{6}$, sendo que um deles é mais próximo do $\sqrt{12} \cong 3,5$ da expressão inicial.
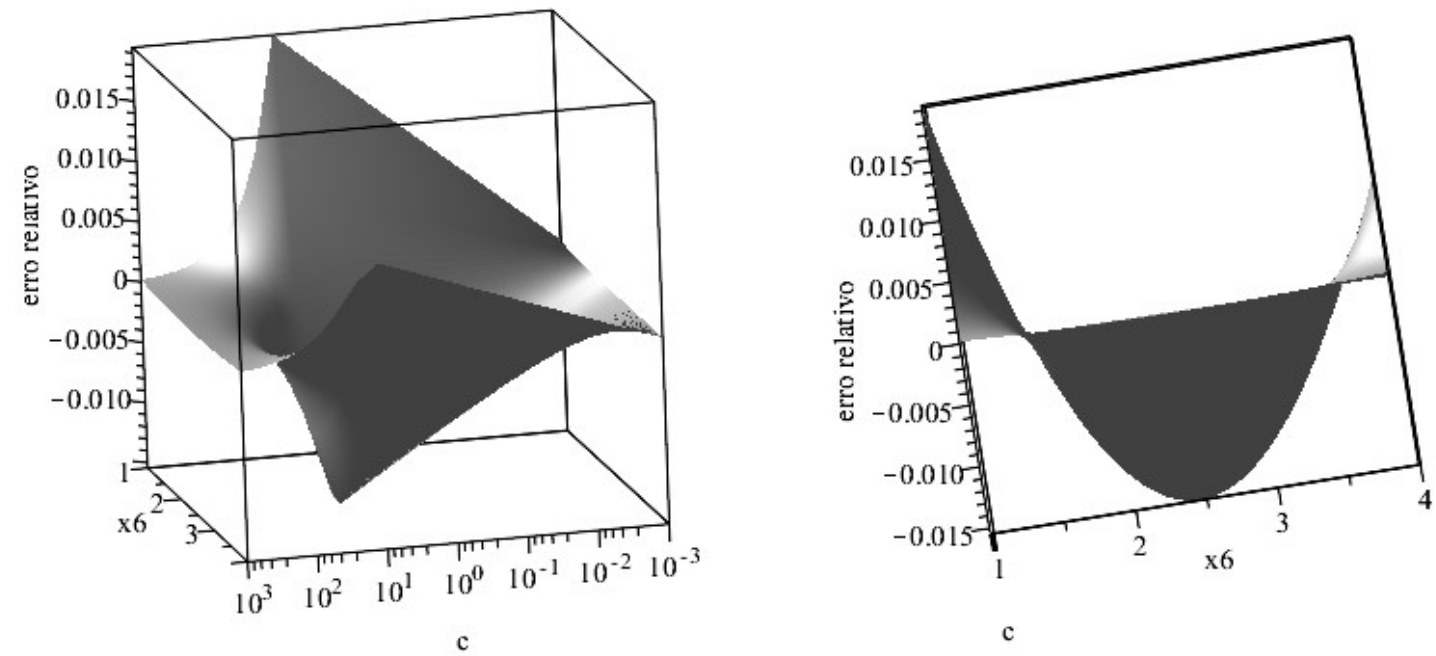

Figura 7.1 Localização dos melhores valores do parâmetro $x_{6}$ para a expressão para as raízes Eq. (1.5) obtida a partir de Padé $[1,1]$

Consecutivos refinamentos nos intervalos levam a $x_{6}=1,59$ e $x_{6}=3,83$. Cada um destes valores é substituído na expressão de $\beta_{n}$, e uma nova busca é feita com o parâmetro resultante através de gráfico análogo, obtendo-se $x_{2}=0,575$ e $x_{2}=2,286$, respectivamente. Substituindo estes parâmetros na expressão de $\beta_{n}$, obtém-se dois conjuntos de resultados que se encontram na Figura 7.2 e na Figura 7.3. 
Considera-se que os resultados da Figura 7.2 são melhores do que os da Figura 7.3, e utilizase, então, $x_{2}=0,575$ e $x_{6}=1,59$. Observando que $x_{6} \cong 1-x_{2} \cong \pi / 2$, é possível se simplificar a expressão de $\beta_{n}$ para:

$$
\beta_{n} \cong \frac{2(n-1)(c+0,57)+\sqrt{c^{2}+c+4(n-1)^{2}}}{2 c / \pi+1}
$$

que tem mesmos parâmetros para qualquer raiz e é bastante simples.

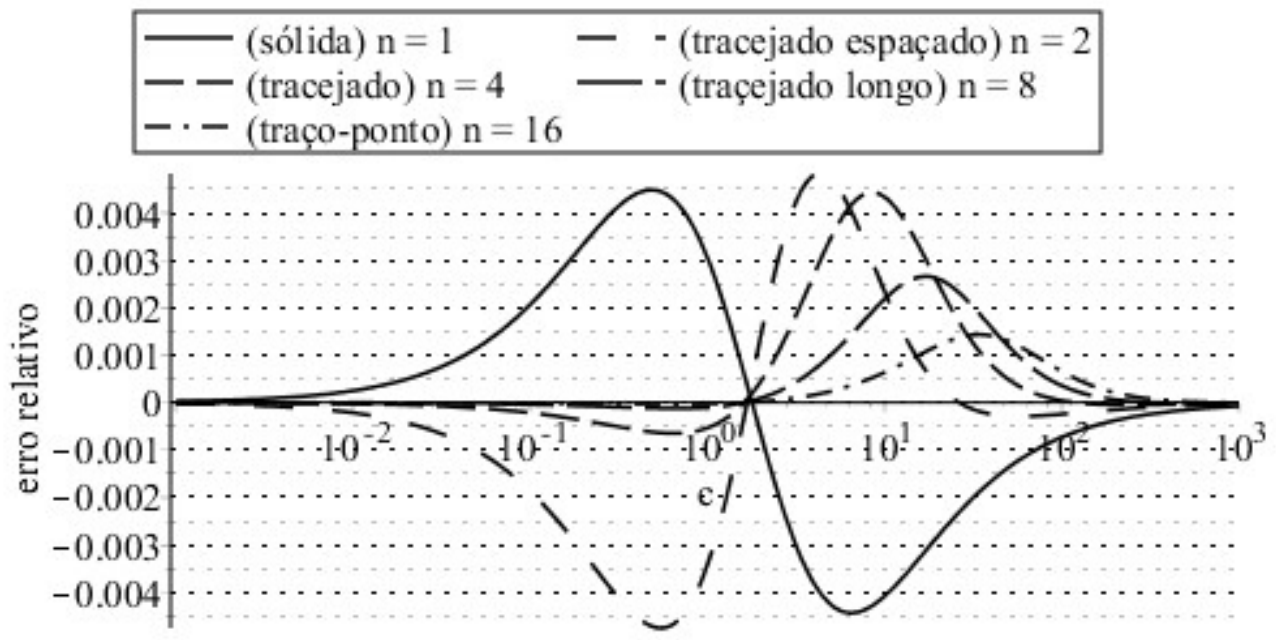

Figura 7.2 Expressão obtida a partir de Padé [1,1] para as raízes Eq. (1.5) com $x_{2}=0,575$ e $x_{6}=1,59$

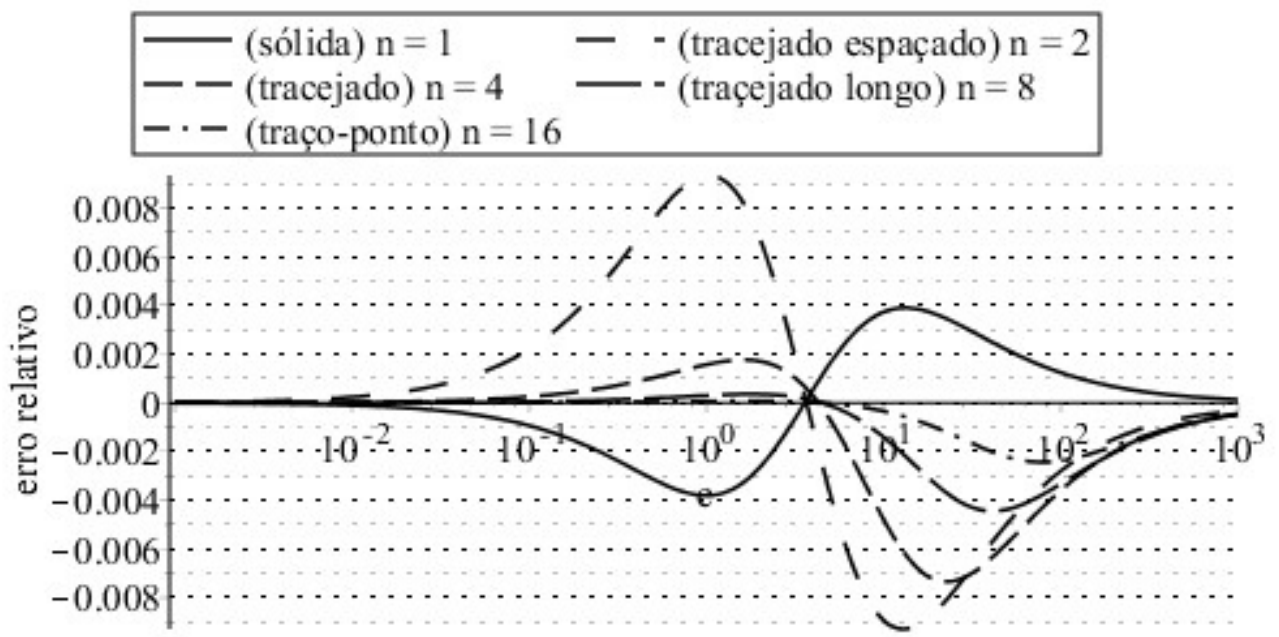

Figura 7.3 Expressão obtida a partir de Padé [1,1] para as raízes Eq. (1.5) com $x_{2}=2,286$ e $x_{6}=3,83$ 
A exatidão da expressão da Eq. (7.1) pode ser vista pela Figura 7.4, que tem como caso particular:

$$
\beta_{1} \cong \frac{\sqrt{c^{2}+c}}{2 c / \pi+1}
$$

que foi utilizada para se encontrar a expressão algébrica da primeira raiz. Expressões análogas para as raízes das outras equações não são possíveis de serem obtidas, e os resultados desta expressão estão mostrados também na Figura 8.18, Figura 8.19 e Figura 8.20, respectivamente para a segunda, a quarta e a oitava da Eq. (1.5).

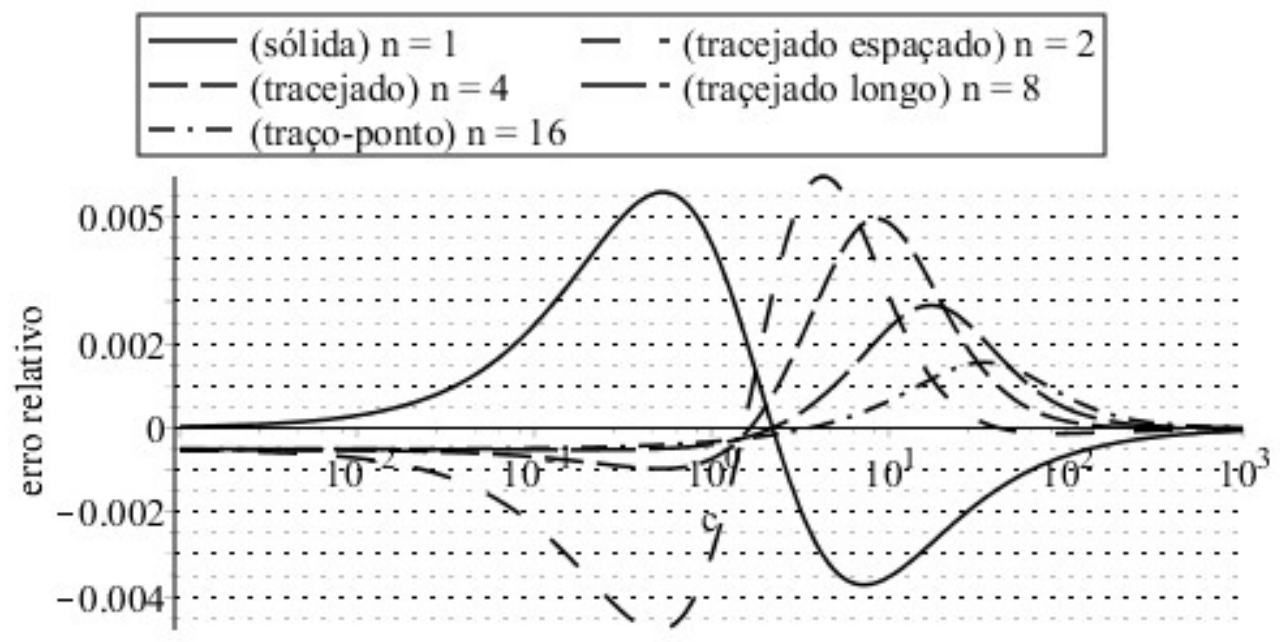

Figura 7.4 Expressão obtida a partir de Padé [1,1] para as raízes Eq. (1.5) com os parâmetros que levam à Eq. (7.1)

\subsection{A Generalização da Expressão de Yovanovich MiniMax}

A expressão Yovanovich MiniMax, mostrada na Eq. (5.4), é uma das que levou aos melhores resultados para a primeira raiz e, então, buscaremos sua generalização para as demais raízes.

Ela foi obtida como aplicação da expressão de Churchill e Usagi (1972):

$$
\beta_{1}(c) \cong \sqrt[r]{\beta_{1,0}^{r}(c)+\beta_{1, \infty}^{r}(c)}
$$

onde $\beta_{1,0}(c)$ e $\beta_{1, \infty}(c)$ são expressões assintóticas para $\beta_{1}$ conforme $c$ tende a zero ou infinito, respectivamente, e $r$ é o parâmetro de ajuste.

Inicialmente observou-se ser necessário que $\beta_{1,0}^{r}(c)$ tenda a zero quando $c$ tender a infinito, e que $\beta_{1, \infty}^{r}(c)$ tenda a zero quando $c$ tender a zero, porque, do contrário, o valor de uma delas iria se somar ao valor da outra e o resultado em cada extremo seria comprometido. Isto faz com que as 
expressões assintóticas $\beta_{1,0}(c)=\sqrt{m c}$ e $\beta_{1, \infty}(c)=\beta_{1, \infty}$ não possam ser aplicadas diretamente, se $r>$ 0 .

Quando é utilizado $k=-r>0$,

$$
\beta_{1}(c) \cong \frac{1}{\sqrt[k]{\frac{1}{\beta_{1,0}^{k}(c)}+\frac{1}{\beta_{1, \infty}^{k}(c)}}}
$$

e as expressões assintóticas acima passam a ser utilizáveis porque quando $c$ tende a zero, o inverso de $\beta_{1,0}^{k}(c)$ aumenta ilimitadamente, e o valor de $\beta_{1, \infty}^{k}(c)$ passa a ser negligenciável. Além disso, quando $c$ tende a infinito, o inverso de $\beta_{1,0}^{k}(c)$ tende a zero e passa a ser ele mesmo negligenciável.

A engenhosidade da expressão de Yovanovich (1996) reside no fato de que o inverso de $\beta_{1,0}^{k}(c)$ tende ora a zero, ora a infinito, enquanto que o inverso de $\beta_{1, \infty}^{k}(c)$ permanece constante, fazendo com que cada um dos termos seja dominante em um dos extremos de $c$.

A questão que será tratada agora é a de como pode ser possível se aproximar as demais raízes, lembrando que elas não tendem nem a zero, nem a infinito, então não há como elas possuírem uma expressão assintótica com esse comportamento.

Isso nos leva a adotar a estratégia de se não utilizar apenas $\beta_{n}(c)$ como função a ser ajustada, mas $\left(\beta_{n}(c)-\beta_{n, 0}\right)$, a qual tende a zero quando $c$ tende a zero, e o objetivo agora passa a ser o de se encontrar uma expressão assintótica para esta função.

A partir da tendência da Eq. (2.13):

$$
\lim _{c \rightarrow 0} \frac{\beta_{n}(c)-\beta_{n, 0}}{c}=\frac{1}{\beta_{n, 0}}
$$

pode-se obter várias expressões assintóticas, sendo que a mais simples é função afim. Então, utilizando esta opção mais simples, e mantendo a outra expressão assintótica como constante, obtémse:

$$
\beta_{n}-\beta_{n, 0} \cong\left(\left(\frac{c}{\beta_{n, 0}}\right)^{r}+\left(\beta_{n, \infty}-\beta_{n, 0}\right)^{r}\right)^{\frac{1}{r}}
$$

O ajuste dessa expressão pelo algoritmo de Remez levou a erros da ordem de $2 \cdot 10^{-3}$, os quais não consideramos aceitáveis, e passamos a investigar outras possibilidades.

Consideremos, inicialmente, o caso particular das raízes da Eq. (1.5), onde $\beta_{n, 0}$ é um múltiplo de $\pi$ e, então:

$$
\tan \left(\beta_{n}-\beta_{n, 0}\right)=\tan \beta_{n}
$$

A aproximação muito conhecida de que: 


$$
\tan \left(\beta_{n}-\beta_{n, 0}\right) \rightarrow \beta_{n}-\beta_{n, 0} \text { quando } \beta_{n}-\beta_{n, 0} \rightarrow 0
$$

leva, então, a

$$
\tan \beta_{n} \rightarrow \beta_{n}-\beta_{n, 0} \text { quando } \beta_{n} \rightarrow \beta_{n, 0}
$$

Multiplicando-se por $\beta_{n}$ ambos lados desta equação, e utilizando a Eq. (1.5), obtém-se:

$$
\beta_{n}(c) \rightarrow \sqrt{c+\left(\frac{\beta_{n, 0}}{2}\right)^{2}}+\frac{\beta_{n, 0}}{2} \text { quando } c \rightarrow 0
$$

onde utilizou-se que $\beta_{n} \rightarrow \beta_{n, 0}$ é equivalente a $c \rightarrow 0$, e que $\beta_{n} \geq 0$.

Observe que essa expressão também satisfaz a Eq. (2.13):

$$
\lim _{c \rightarrow 0} \frac{\sqrt{c+\left(\frac{\beta_{n, 0}}{2}\right)^{2}}+\frac{\beta_{n, 0}}{2}-\beta_{n, 0}}{c}=\lim _{c \rightarrow 0} \frac{\sqrt{c+\left(\frac{\beta_{n, 0}}{2}\right)^{2}}-\frac{\beta_{n, 0}}{2}}{c}=\lim _{c \rightarrow 0} \frac{1}{\sqrt{c+\left(\frac{\beta_{n, 0}}{2}\right)^{2}}+\frac{\beta_{n, 0}}{2}}=\frac{1}{\beta_{n, 0}}
$$

e, utilizando ela como nova expressão assintótica, obtém-se:

$$
\beta_{n} \cong \beta_{n, 0}+\left(\left(\sqrt{c+\left(\frac{\beta_{n, 0}}{2}\right)^{2}}-\frac{\beta_{n, 0}}{2}\right)^{r}+\left(\beta_{n, \infty}-\beta_{n, 0}\right)^{r}\right)^{\frac{1}{r}}
$$

que generaliza a expressão de Yovanovich pois $\beta_{1,0}=0$. Já se adianta que o ajuste dessa expressão levará a resultados satisfatórios, e passamos a procurar formas análogas para as raízes das outras equações.

A função cotangente não tem uma expansão de Taylor em torno de zero para ser possível se repetir a dedução acima. É possível se modificar a Eq. (1.7) para:

$$
\frac{1}{\beta} \tan \beta=\frac{1}{1-c}
$$

e utilizar novamente que:

$$
\tan \beta_{n} \rightarrow \beta_{n}-\beta_{n, 0} \text { quando } \beta_{n} \rightarrow \beta_{n, 0}
$$

multiplicando-se ambos seus lados por $1 / \beta_{n}$ para obter:

$$
\beta_{n}(c) \rightarrow \beta_{n, 0}\left(1-\frac{1}{c}\right) \text { quando } c \rightarrow 0
$$

que poderia ser utilizada para uma dedução análoga, porém seu resultado não corresponderia à expressão de Yovanovich para $n=1.0$ caso da Eq. (1.6) seria ainda pior, porque as funções de Bessel não têm periodicidade, o que foi base para a dedução acima. Investigaremos, então, outra maneira.

No caso particular $n=1$, para a Eq. (1.5), Yovanovich (1996) obteve: 


$$
\beta_{1} \cong\left((\sqrt{c})^{r}+\left(\beta_{n, \infty}\right)^{r}\right)^{\frac{1}{r}}
$$

enquanto que, no caso geral das Eqs. (1.5), (1.6) e (1.7), ele obteve:

$$
\beta_{1} \cong\left((\sqrt{m c})^{r}+\left(\beta_{n, \infty}\right)^{r}\right)^{\frac{1}{r}}
$$

Pois bem, para a Eq. (1.5), demonstrou-se que:

$$
\beta_{n} \cong \beta_{n, 0}+\left(\left(\sqrt{c+\left(\frac{\beta_{n, 0}}{2}\right)^{2}}-\frac{\beta_{n, 0}}{2}\right)^{r}+\left(\beta_{n, \infty}-\beta_{n, 0}\right)^{r}\right)^{\frac{1}{r}}
$$

e agora é necessária uma expressão equivalente no caso geral.

O grande número de vezes que foram obtidos resultados análogos para as três equações torna tentador investigar se a expressão genérica pode ser encontrada apenas substituindo $c$ por $m c$. Observe-se como a expressão de Yovanovich, no caso da Eq. (1.5), é o caso particular correspondente a $\beta_{1,0}=0$, o que demonstra muita analogia entre essas três expressões.

Porém, a dúvida passa a ser sobre como deve surgir o termo $\beta_{n, 0} / 2$ na expressão equivalente ao caso geral. Deixando esse termo como um parâmetro livre dentro do algoritmo de Remez, para encontrar quais são seus valores ótimos, obtém-se os resultados que estão apresentados na Tabela 7.1 e se observa que ele se aproxima de $m \beta_{n, 0} / 2$.

Tabela 7.1 Busca de expressão para um parâmetro ótimo da expressão de Yovanovich MiniMax Generalizada

\begin{tabular}{ccccccc}
\hline & \multicolumn{2}{c}{ Eq. (1.5) } & \multicolumn{2}{c}{ Eq. (1.6) } & \multicolumn{2}{c}{ Eq. (1.7) } \\
\cline { 2 - 5 }$n$ & $\begin{array}{c}\text { Parâmetro } \\
\text { ótimo }\end{array}$ & $\frac{m \beta_{n, 0}}{2}$ & $\begin{array}{c}\text { Parâmetro } \\
\text { ótimo }\end{array}$ & $\frac{m \beta_{n, 0}}{2}$ & $\begin{array}{c}\text { Parâmetro } \\
\text { ótimo }\end{array}$ & $\frac{m \beta_{n, 0}}{2}$ \\
\hline 2 & 1,55 & 1,57 & 3,73 & 3,83 & 6,33 & 6,74 \\
3 & 3,05 & 3,14 & 6,67 & 7,02 & 10,8 & 11,6 \\
4 & 4,50 & 4,71 & 9,55 & 10,2 & 15,0 & 16,4 \\
5 & 5,97 & 6,28 & 12,4 & 13,3 & 19,4 & 21,1 \\
6 & 7,40 & 7,85 & 15,2 & 16,5 & 23,5 & 25,8 \\
7 & 8,82 & 9,42 & 18,1 & 19,6 & 27,7 & 30,6 \\
8 & 10,3 & 11,0 & 20,9 & 22,8 & 32,1 & 35,3 \\
9 & 11,7 & 12,6 & 23,7 & 25,9 & 36,2 & 40,0 \\
\hline
\end{tabular}

Para o caso da Eq. (1.5), o parâmetro ótimo se aproxima do termo deduzido $\beta_{n, 0} / 2$, e para as outras equações, encontrou-se que $m \beta_{n, 0} / 2$ também é uma boa aproximação. Então, a expressão generalizada escolhida é: 


$$
\beta_{n} \cong \beta_{n, 0}+\left(\left(\sqrt{m c+\left(\frac{m \beta_{n, 0}}{2}\right)^{2}}-\frac{m \beta_{n, 0}}{2}\right)^{r}+\left(\beta_{n, \infty}-\beta_{n, 0}\right)^{r}\right)^{\frac{1}{r}}
$$

a qual foi ajustada aos valores exatos das raízes das Eqs. (1.5), (1.6) e (1.7) e tem parâmetros que foram obtidos através do algoritmo de Remez apresentados na Tabela 7.2.

Essa expressão possui valores corretos quando $c$ tende a infinito:

$$
\lim _{c \rightarrow \infty}\left(\beta_{n, 0}+\left(\left(\sqrt{m c+\left(\frac{m \beta_{n, 0}}{2}\right)^{2}}-\frac{m \beta_{n, 0}}{2}\right)^{r}+\left(\beta_{n, \infty}-\beta_{n, 0}\right)^{r}\right)^{\frac{1}{r}}\right)=\beta_{n, \infty}
$$

e também possui a tendência da Eq. (2.13):

$$
\lim _{c \rightarrow 0} \frac{\beta_{n, 0}+\left(\left(\sqrt{m c+\left(\frac{m \beta_{n, 0}}{2}\right)^{2}}-\frac{m \beta_{n, 0}}{2}\right)^{r}+\left(\beta_{n, \infty}-\beta_{n, 0}\right)^{r}\right)^{\frac{1}{r}}-\beta_{n, 0}}{c}=\frac{1}{\beta_{n, 0}}
$$

então ela tem erro tendendo a zero quando $c$ tende a zero ou infinito, conforme pode ser visto desde a Figura 8.27 até a Figura 8.35, onde ela está identificada como "Yovanovich MiniMax Generalizada”.

\begin{tabular}{cccc}
\multicolumn{4}{c}{ Tabela 7.2 Parâmetro r da Eq. (7.3) } \\
\hline & Eq. (1.5) & Eq. (1.6) & Eq. (1.7) \\
\hline 1 & $-2,140$ & $-2,247$ & $-2,334$ \\
2 & $-1,740$ & $-1,612$ & $-1,640$ \\
3 & $-1,650$ & $-1,561$ & $-1,572$ \\
4 & $-1,607$ & $-1,538$ & $-1,543$ \\
5 & $-1,581$ & $-1,524$ & $-1,527$ \\
6 & $-1,564$ & $-1,514$ & $-1,516$ \\
7 & $-1,551$ & $-1,508$ & $-1,509$ \\
8 & $-1,541$ & $-1,502$ & $-1,503$ \\
9 & $-1,533$ & $-1,499$ & $-1,499$ \\
\hline
\end{tabular}

\subsection{Uma Expressão Algébrica}

As demais raízes das Eqs. (1.5), (1.6) e (1.7) não possuem a tendência da Eq. (2.12), então uma forma diferente da expressão algébrica que foi utilizada para a primeira raiz, deve ser utilizada para elas. 
Inspirando-se na forma que levou a bons resultados para a primeira raiz, utilizamos uma expressão que novamente não é racional por ter potências que não são inteiras:

$$
\beta_{n} \cong \frac{A \beta_{n, 0}+\beta_{n, \infty} c^{k}}{A+c^{k}}
$$

Esta expressão não satisfaz a tendência dada pela Eq. (2.13), mas tem valores corretos quando $c$ tende a zero ou infinito, como pode ser facilmente observado e, portanto, tem erro relativo tendendo a zero nesses dois extremos.

Aplicando-se o algoritmo de Remez, obtém-se os valores da Tabela 7.3. Os resultados desta expressão podem ser vistos desde a Figura 8.27 até a Figura 8.35.

Tabela 7.3 Parâmetros $k$ e A da Eq. (7.4)

\begin{tabular}{ccccccc}
\hline \multirow{2}{*}{$n$} & \multicolumn{2}{c}{ Eq. (1.5) } & \multicolumn{2}{c}{ Eq. (1.6) } & \multicolumn{2}{c}{ Eq. (1.7) } \\
\cline { 2 - 7 } & $k$ & $A$ & $k$ & $A$ & $k$ & $A$ \\
\hline 2 & 1,10 & 4,49 & 1,15 & 5,80 & 1,18 & 7,20 \\
3 & 1,15 & 9,44 & 1,17 & 11,1 & 1,20 & 13,1 \\
4 & 1,17 & 15,1 & 1,18 & 16,9 & 1,20 & 19,2 \\
5 & 1,18 & 21,2 & 1,19 & 23,4 & 1,21 & 26,1 \\
6 & 1,19 & 28,0 & 1,20 & 30,4 & 1,21 & 32,9 \\
7 & 1,19 & 34,5 & 1,20 & 37,2 & 1,21 & 40,2 \\
8 & 1,19 & 41,3 & 1,20 & 44,3 & 1,21 & 47,4 \\
9 & 1,20 & 49,5 & 1,20 & 51,5 & 1,21 & 55,0 \\
\hline
\end{tabular}

\subsection{A Generalização da Expressão que Utiliza Ponto Fixo}

Sabe-se que a função tangente tem período $\pi$ e, então, qualquer que seja $n$ inteiro:

$$
y=\tan x=\tan (x+n \pi)
$$

Deste modo, para ser invertida, ela tem seu domínio restrito a $(-\pi / 2, \pi / 2)$ e, consequentemente, sua inversa tem esse intervalo como imagem:

$$
-\frac{\pi}{2}<x=\arctan y<\frac{\pi}{2}
$$

Então, para se ter o valor de $x$ que está no intervalo $(-\pi / 2, \pi / 2)$ e que tem tangente igual a $y$, utiliza-se a função tangente inversa (ou arco tangente) e, para se ter os demais valores de $x$ que tem tangente igual a $y$, é necessário somar múltiplos de $\pi$ à tangente inversa de $y$ :

$$
x=\arctan y+(n-1) \pi
$$

Deste modo, devemos manter em mente que

$$
y=\tan x \rightarrow x=\arctan y+(n-1) \pi
$$


Da Eq. (1.5), obtém-se:

$$
\tan \beta_{n}=\frac{c}{\beta_{n}}
$$

então:

$$
\beta_{n}=\arctan \frac{c}{\beta_{n}}+(n-1) \pi
$$

A expressão para a primeira raiz já foi obtida, e agora será necessário uma estimativa válida para as demais raízes que possa substituir o $\beta_{n}$ do lado direito desta equação. A expressão da Eq. (2.20) é bastante simples e leva a

$$
\beta_{n}=\arctan \frac{c}{c_{n}+\frac{\pi}{4}\left(\frac{c-c_{n}}{c+c_{n}}\right)}+(n-1) \pi
$$

onde

$$
c_{n}=\left(n-\frac{3}{4}\right) \pi
$$

Simplificando-se esta expressão, obtém-se:

$$
\beta_{n} \cong \arctan \frac{c}{\left(n-\frac{1}{2}\right) \pi-\frac{\pi}{2+\frac{2 c / \pi}{n-3 / 4}}}+(n-1) \pi
$$

Ao contrário expressão da Eq. (2.20), esta expressão satisfaz a tendência dada pela Eq. (2.13), o que pode ser visto por cálculo elementar, através de:

$$
\lim _{c \rightarrow 0} \frac{\beta_{n}(c)-\beta_{n, 0}}{c}=\frac{1}{(n-1) \pi}=\frac{1}{\beta_{n, 0}}
$$

e também tem valor correto quando $c$ tende a infinito:

$$
\lim _{c \rightarrow \infty} \beta_{n}(c)=\left(n-\frac{1}{2}\right) \pi=\beta_{n, \infty}
$$

Portanto, esta expressão tem erro relativo tendendo a zero quando $c$ tende a infinito. Observese na Figura 8.27, Figura 8.28, Figura 8.29 para as raízes $\beta_{2}, \beta_{4}$ e $\beta_{8}$, respectivamente, erros dessa expressão que que diminui muito rapidamente de uma raiz para outra.

O procedimento acima pode ser repetido de maneira absolutamente análoga para a Eq. (1.7), e isto não será aqui repetido, mas apenas apresentado o resultado: 


$$
\beta_{n} \cong \operatorname{arccot} \frac{1-c}{\left(n-\frac{1}{2}\right) \pi-\frac{1-c \frac{\pi}{2}}{(n-1 / 2) \pi+c}}+(n-1) \pi
$$

a qual tem sucessão de erros que diminui ainda mais rapidamente de uma raiz para outra, conforme pode ser visto na Figura 8.33, Figura 8.34 e Figura 8.35, para as raízes $\beta_{2}, \beta_{4}$ e $\beta_{8}$, respectivamente. Note-se que a última curva está quase se confundindo com o eixo horizontal do gráfico e essas expressões sequer inclui um parâmetro de ajuste.

Porém, estas expressões apresentam resultados apenas medianos para a primeira raiz e, devido às várias limitações já citadas sobre as funções de Bessel, não há como se obter uma expressão análoga para as raízes da Eq. (1.6). 


\section{RESULTADOS E DISCUSSÃO}

Analisaremos os resultados de todas expressões aqui desenvolvidas, comparando-as com as já existentes. A simplicidade das expressões poderá ser observada por elas estarem repetidas aqui dentro de seções, e suas exatidões poderão ser observadas através de gráficos.

Dividiu-se as expressões desenvolvidas neste e em outros trabalhos em dois grupos, o primeiro formado pelas expressões válidas para a primeira raiz, e o segundo com as expressões válidas para as demais raízes.

0 primeiro grupo foi dividido em seis seções, conforme suas exatidões e em cada seção apresentou-se gráficos relativos a cada uma das Eqs. (1.5), (1.6) e (1.7), resultando em 17 gráficos mostrados da Figura 8.1 à Figura 8.17 (a última figura inclui duas equações).

O segundo grupo foi dividido em apenas duas seções, conforme suas exatidões, e em cada seção apresentou-se gráficos relativos a segunda, quarta e oitava raiz de cada uma das Eqs. (1.5), (1.6) e (1.7), resultando em 18 gráficos mostrados da Figura 8.18 à Figura 8.35. Esta numeração em progressão geométrica que está sempre sendo escolhida neste trabalho dá mais destaque as raízes de ordem inferior, sem deixar de mostrar as de ordem superior. Note-se que os erros têm tendência de diminuírem conforme a ordem aumenta.

Como erros negativos tem mesmos significados do que erros positivos, para facilitar as comparações, é utilizado o módulo do erro relativo, evidenciando assim o que tiver maior magnitude.

Também para facilitar as comparações, utilizou-se mesmos tipos de linhas (contínua, tracejada, pontilhada e tracejado-pontilhada) para cada trabalho dentro da mesma seção. Todos gráficos tiveram eixos horizontais logarítmicos, com escala de $10^{-3}$ a $10^{3}$, sendo que apenas os da primeira seção tiveram eixos verticais também logarítmicos, para comportarem toda a variação de seus valores.

Seria conveniente que estes gráficos também incluíssem o erro das raízes calculados por tabelas, para efeito de comparação, porém ele é muito oscilante e dificultaria a visualização das demais curvas, então deve-se manter em mente seus valores que foram apresentados durante a discussão das tabelas: $2 \%$ para as de passo variável e de até 0,5\% para as de passo constante, se forem utilizadas interpolações de grande ordem, e não se utilizar valores baixos de $c$. 


\subsection{Expressões de Muito Menor Exatidão para a Primeira Raiz}

As expressões de Stevens e Luck (1999) mostradas na Eqs. (2.20), (2.21) e (2.22):

$$
\begin{gathered}
\beta_{1} \cong \frac{\pi}{4}+\frac{\pi}{4} \frac{c-\frac{\pi}{4}}{c+\frac{\pi}{4}} \\
\beta_{1} \cong \frac{3 \pi}{8}+\frac{3 \pi}{8} \frac{c-\frac{\pi}{2}}{c+\frac{\pi}{2}} \\
\beta_{1} \cong \frac{\pi}{2}+\frac{\pi}{2} \frac{c^{0,676}-\frac{\sqrt{\pi}}{2}}{c^{0,676}+\frac{\sqrt{\pi}}{2}}
\end{gathered}
$$

para a primeira raiz das Eqs. (1.5), (1.6) e (1.7), respectivamente, podem ser reescritas como uma única expressão:

$$
\beta_{1}=p \frac{c^{q}}{c^{q}+r}
$$

onde $p=\pi / 2,3 \pi / 4, \pi, q=1,1,0,676$ e $r=\pi / 4, \pi / 2, \sqrt{\pi} / 2$ para as Eqs. (1.5), (1.6) e (1.7), respectivamente. Elas são bastante simples mas tem erro que tende a 100\% quando $c$ tende a zero.

A expressão de Haji-Sheik e Beck (2000) mostrada na Eq. (2.23):

$$
\beta_{1} \cong \frac{\pi}{4}+\frac{\pi}{4}\left(1-1,04\left(\sqrt{\frac{c}{c+0,76}}-\frac{c}{c+0,76}\right)\right) \frac{c-\frac{\pi}{4}}{c+\frac{\pi}{4}}
$$

para a primeira raiz da Eq. (1.5) tem erro que tende a 6,4\% (em módulo) quando $c$ tende a zero, e a expressão mostrada na Eq. (2.24):

$$
\beta_{1} \cong \frac{3 \pi}{4}+\frac{\pi}{4} \frac{c-1-\frac{3 \pi}{4}}{c-1+\frac{3 \pi}{4}}
$$

para a primeira raiz da Eq. (1.7), tem erro ilimitado quando $c$ tende a zero, além de não terem uma versão análoga para a Eq. (1.6). 0 objetivo de se desenvolver estas expressões foi o de se melhorar as expressões de Stevens e Luck (1999), porém, no caso da primeira, a melhor exatidão foi conseguida com diminuição muito grande da simplicidade e, no caso da segunda, a melhor exatidão não foi conseguida em todo o intervalo. 


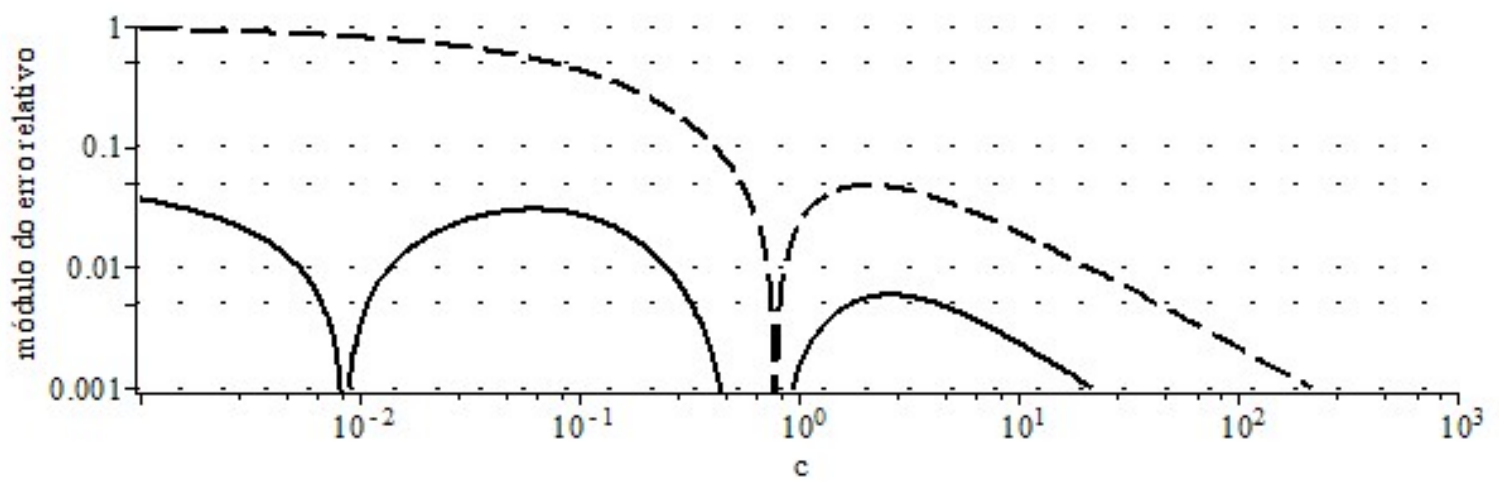

\section{Stevens e Luck (1999) - Haji-Sheikh e Beck (2000)}

Figura 8.1 Expressões de muito menor exatidão para a primeira raiz da Eq. (1.5)

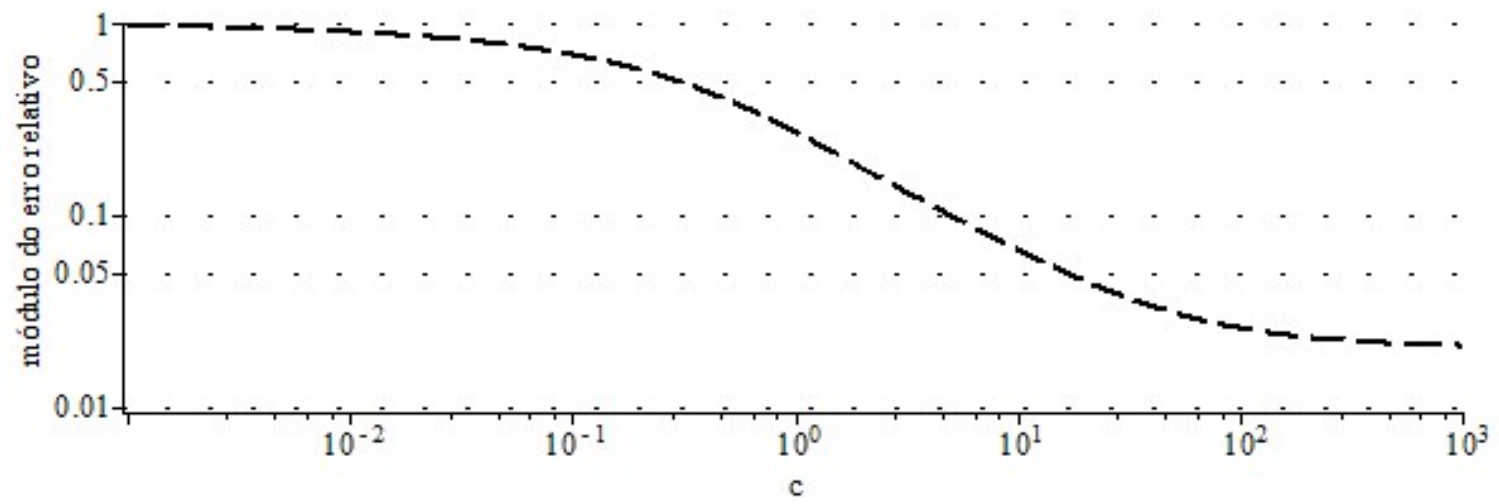

Stevens e Luck (1999)

Figura 8.2 Expressões de muito menor exatidão para a primeira raiz da Eq. (1.6)

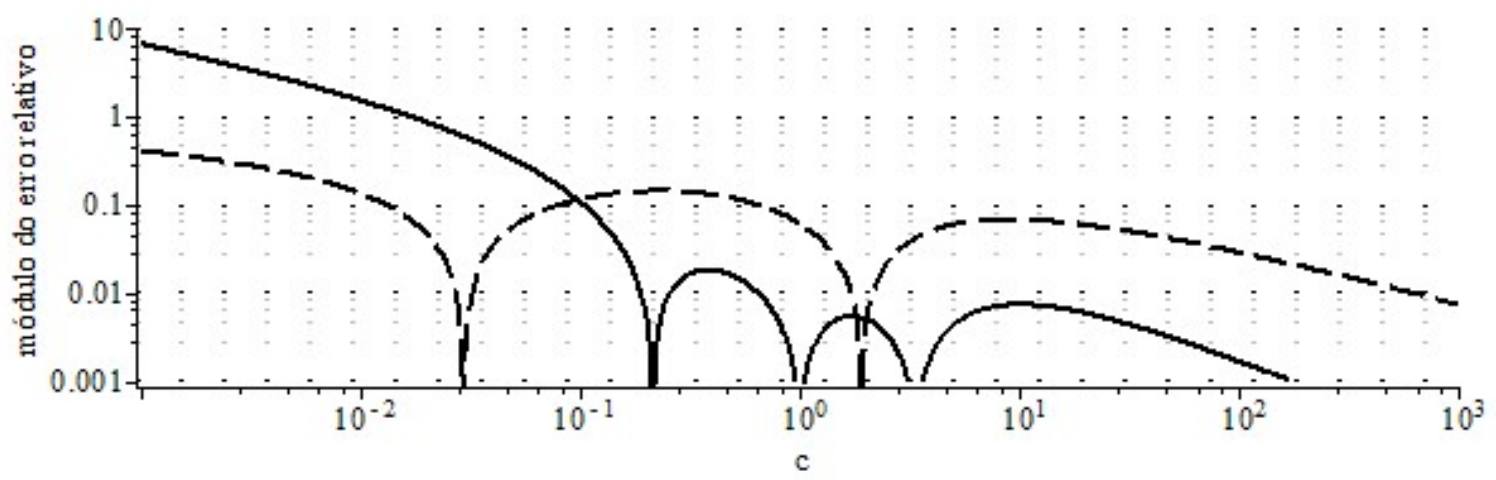

Stevens e Luck (1999) - Haji-Sheikh e Beck (2000)

Figura 8.3 Expressões de muito menor exatidão para a primeira raiz da Eq. (1.7) 


\subsection{Expressões de Menor Exatidão para a Primeira Raiz}

A expressão de Luikov (1968) mostrada na Eq. (2.14):

$$
\beta_{1} \cong \frac{\beta_{1, \infty}}{\sqrt{1+A / c^{k}}}
$$

onde $A=2,24,2,45$ e 2,70, e $k=1,02,1,04$ e 1,07, para a primeira raiz das Eqs. (1.5), (1.6) e (1.7), respectivamente, também pode ser escrita como:

$$
\beta_{1} \cong\left(B c^{-k}+\beta_{1, \infty}^{-2}\right)^{-\frac{1}{2}}
$$

onde $B=0,908,0,424,0,273$, respectivamente. Ela também tem erro que tende a $100 \%$ quando $c$ tende a zero, porém este aumento do erro é lento e visualizável apenas com valores de $c$ muito menores do que os que aparecem na escala escolhida para os gráficos $\left(10^{-3}\right.$ a $\left.10^{3}\right)$,

A expressão de Ostrogorsky e Mikic (2008) mostrada na Eq. (2.25):

$$
\beta_{1} \cong \sqrt{\frac{m c}{1+\frac{m c}{\beta_{1, \infty}^{2}}}}
$$

para a primeira raiz das Eqs. (1.5), (1.6) e (1.7), também pode ser escrita como:

$$
\beta_{1} \cong\left((m c)^{-1}+\beta_{1, \infty}^{-2}\right)^{-\frac{1}{2}}
$$

e é bem melhor do que as expressões dos três trabalhos acima por ter erro relativo tendendo a zero para os dois lados com valores máximos de 2,5\% a 5\%, ser válida para as três equações, e ser tão simples a ponto de não ter nem mesmo parâmetros de ajuste, mas apenas incluir os valores de $\beta_{1, \infty} \mathrm{e}$ $m$.

As expressões de Ostrogorsky e Mikic (2009) mostrada na Eq. (2.26):

$$
\beta_{1} \cong\left\{\begin{array}{l}
\sqrt{\frac{m c}{1+\frac{c}{m+2}}}, 0 \leq c<2 \\
\beta_{1, \infty} \frac{c}{c+a}, c \geq 2
\end{array}\right.
$$

onde $a=0,95,1,0$ e 1,1, para as Eqs. (1.5), (1.6) e (1.7), respectivamente, tem erros de 1,7\% a 2\%, que consideramos altos para sua complexidade (divisão do intervalo de $c$, e inclusão de parâmetro de ajuste).

A expressão de Ostrogorsky (2010a) mostrada na Eq. (2.27): 


$$
\beta_{1} \cong\left\{\begin{array}{l}
\sqrt{m c}, 0 \leq c \leq 0.1 \\
\frac{\beta_{1, \infty}}{\sqrt{1+\frac{a}{c^{k}}}}, c>0.1
\end{array}\right.
$$

onde $a=2,3,2,62$ e 2,92, e $k=1,035,1,05$ e 1,08 para a primeira raiz das Eqs. (1.5), (1.6) e (1.7), respectivamente, tem erros máximos de $0,7 \%$ a 4\%, que também consideramos altos. 


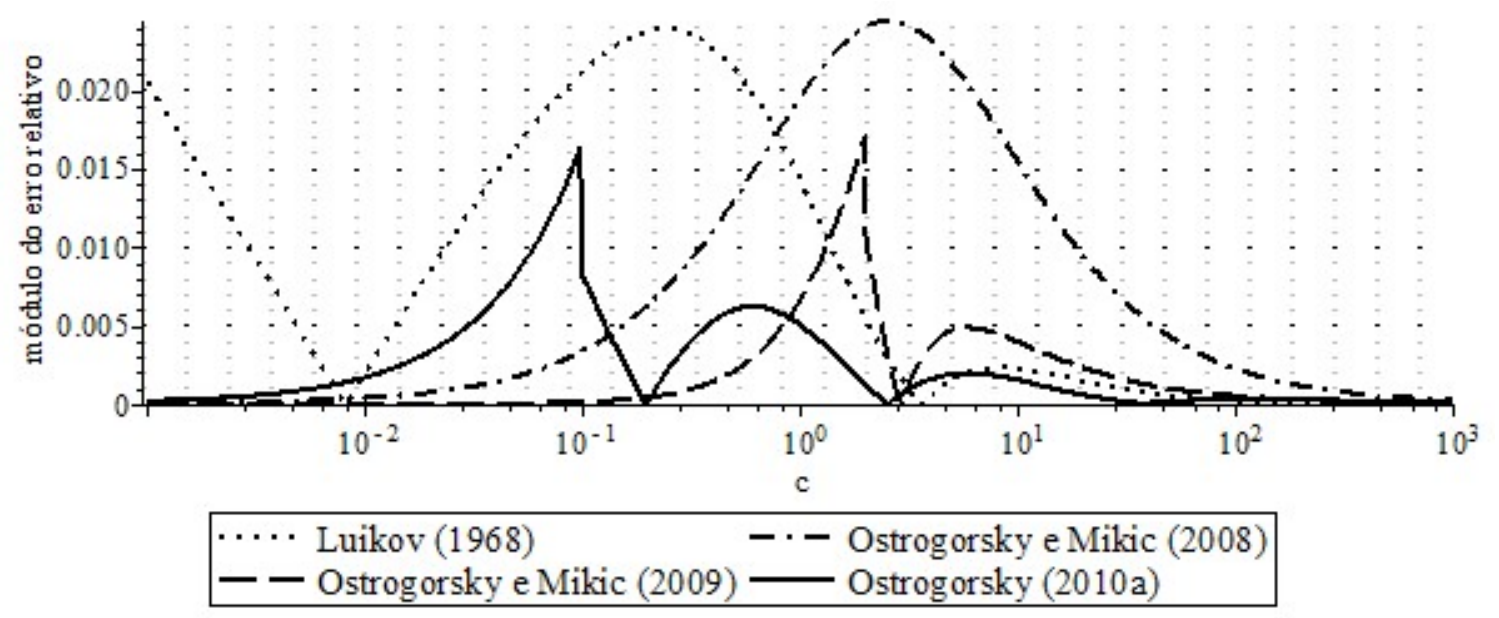

Figura 8.4 Expressões de menor exatidão para a primeira raiz da Eq. (1.5)

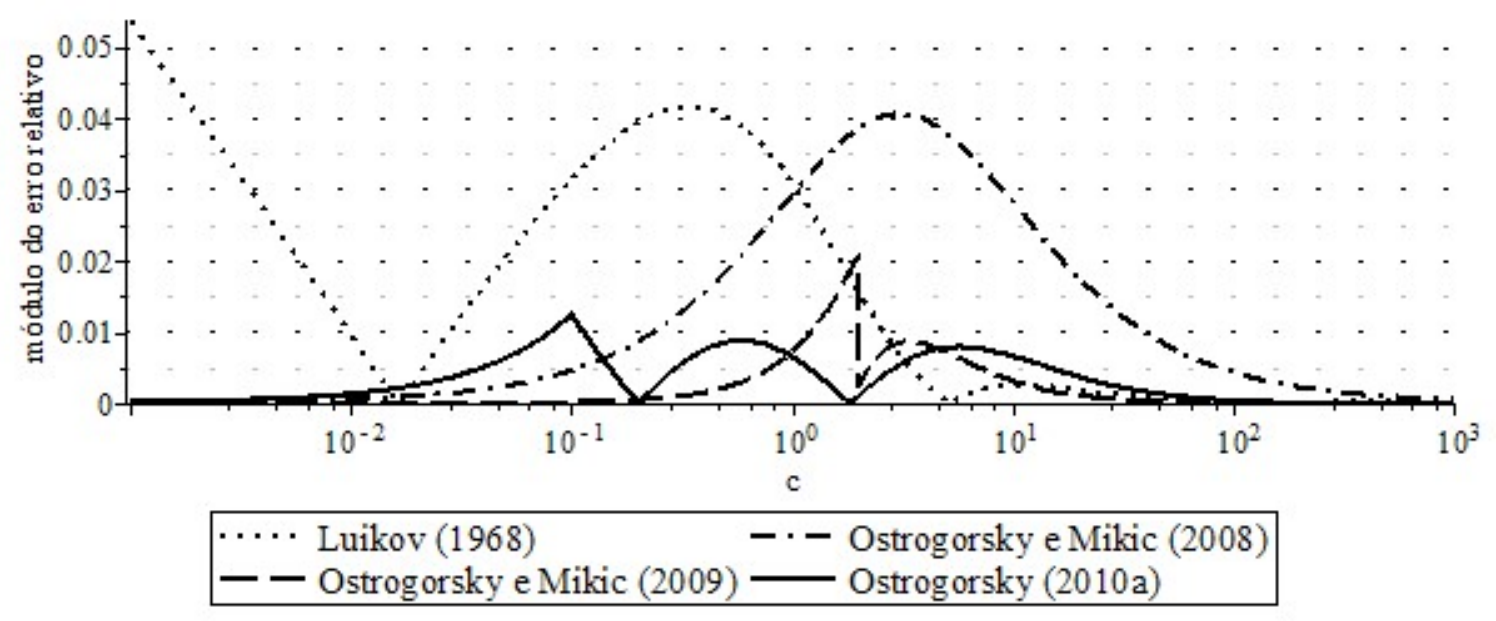

Figura 8.5 Expressões de menor exatidão para a primeira raiz da Eq. (1.6)

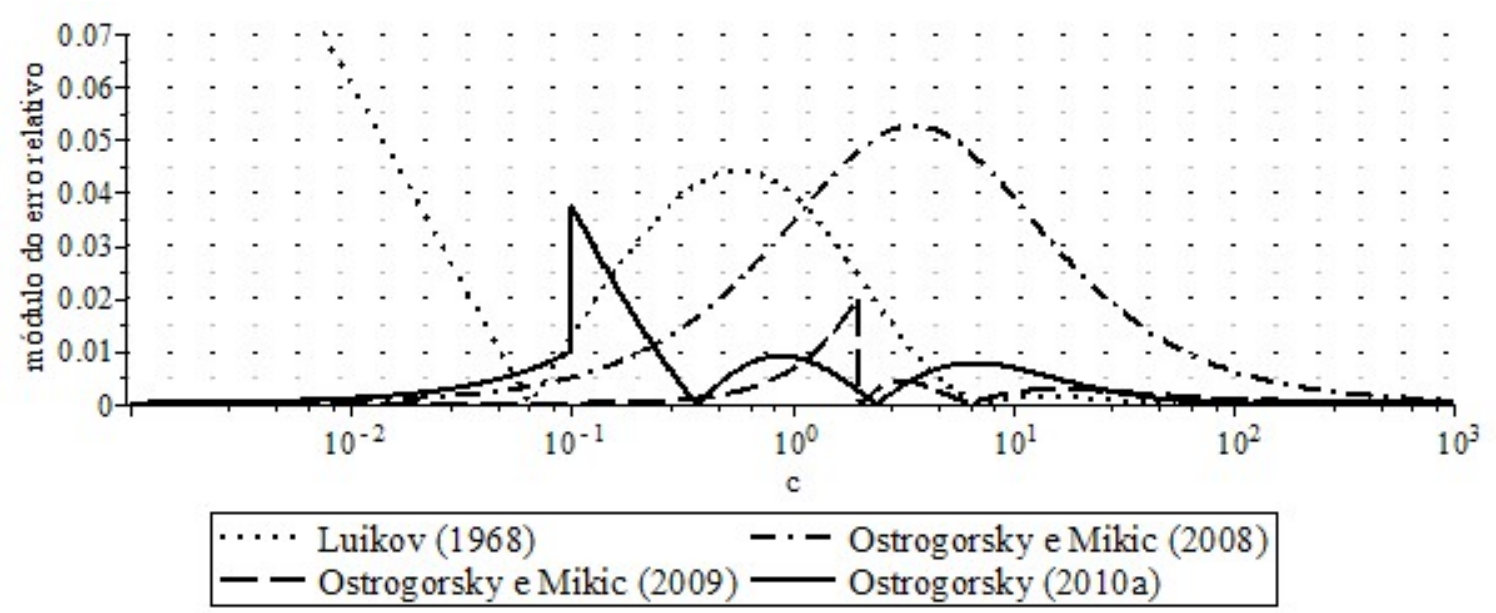

Figura 8.6 Expressões de menor exatidão para a primeira raiz da Eq. (1.7) 


\subsection{Expressões de Intermediária Exatidão para a Primeira Raiz}

A expressão obtida no presente trabalho a partir do ponto fixo mostrada na Eq. (5.1):

$$
\beta_{1} \cong \arctan \sqrt{c+\frac{4 c^{2}}{\pi^{2}}}
$$

para a primeira raiz da Eq. (1.5), e a expressão mostrada na Eq. (5.2):

$$
\beta_{1} \cong \operatorname{arccot}\left(\frac{1-c}{\pi} \sqrt{1+\frac{\pi^{2}}{3 c}}\right)
$$

para a primeira raiz da Eq. (1.7), tem erro máximo de 1,1\%, e são simples a ponto de não ter parâmetro de ajuste, mas não tem uma versão análoga para a Eq. (1.6).

A expressão de Ostrogorsky (2010b) mostrada na Eq. (2.28):

$$
\beta_{1} \cong \frac{\beta_{1, \infty}}{\left(1+\frac{a}{c^{k}}\right)^{d}}
$$

que também pode ser escrita como:

$$
\beta_{1} \cong \beta_{1, \infty}\left(1+a c^{-k}\right)^{-d}
$$

onde $a=2,62,3,28$ e 4,1, $k=1,07,1,125$ e 1,18, e $d=0,468,0,446$ e 0,4238 para as Eqs. (1.5), (1.6) e (1.7), respectivamente, tem erro que cresce lentamente mas tende a $100 \%$ quando $c$ tende a zero. 


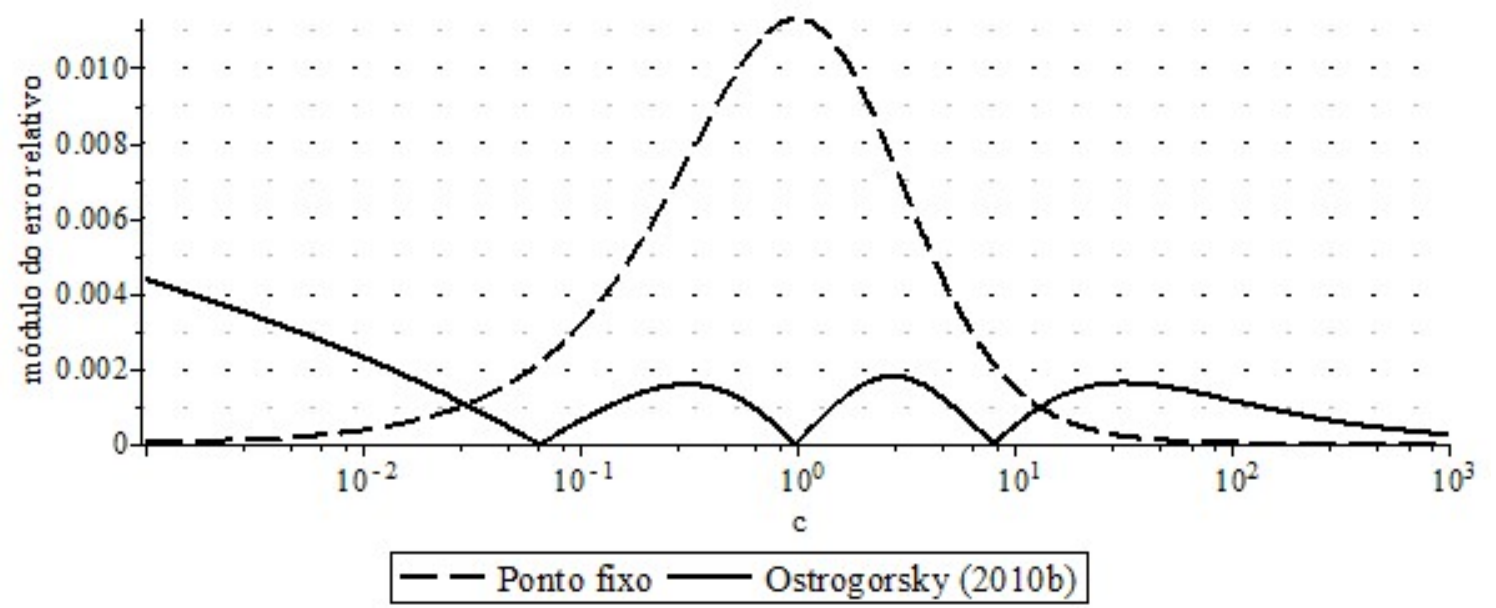

Figura 8.7 Expressões de intermediária exatidão para a primeira raiz da Eq. (1.5)

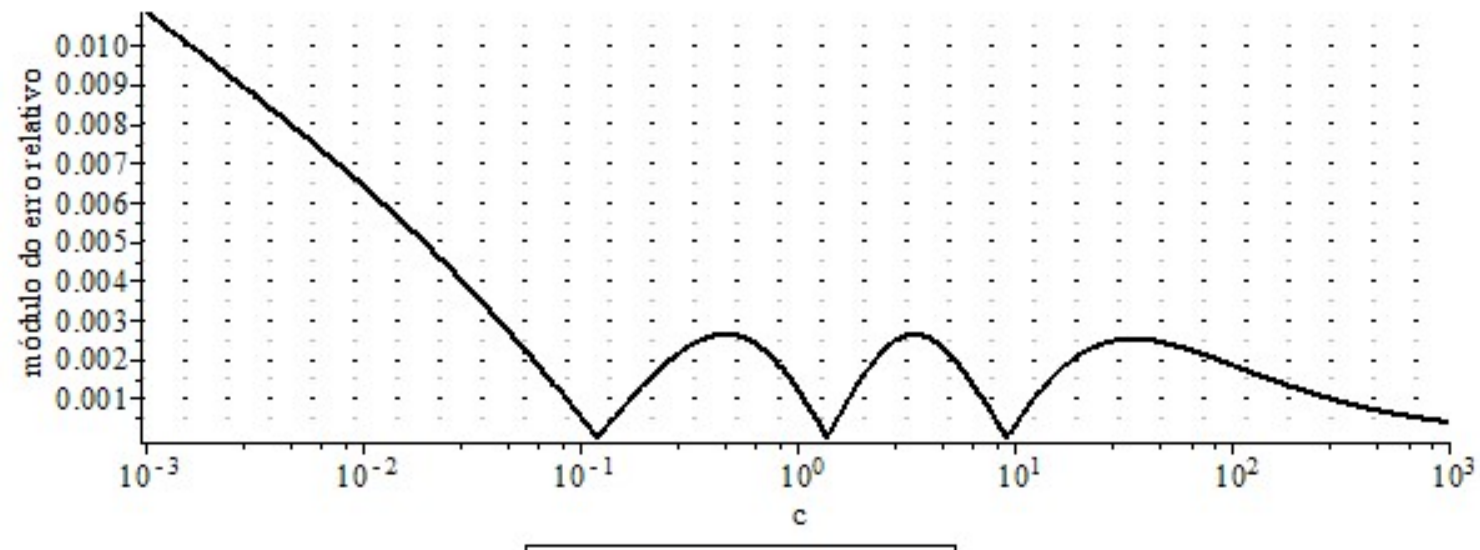

Ostroborsky (2010b)

Figura 8.8 Expressões de intermediária exatidão para a primeira raiz da Eq. (1.6)

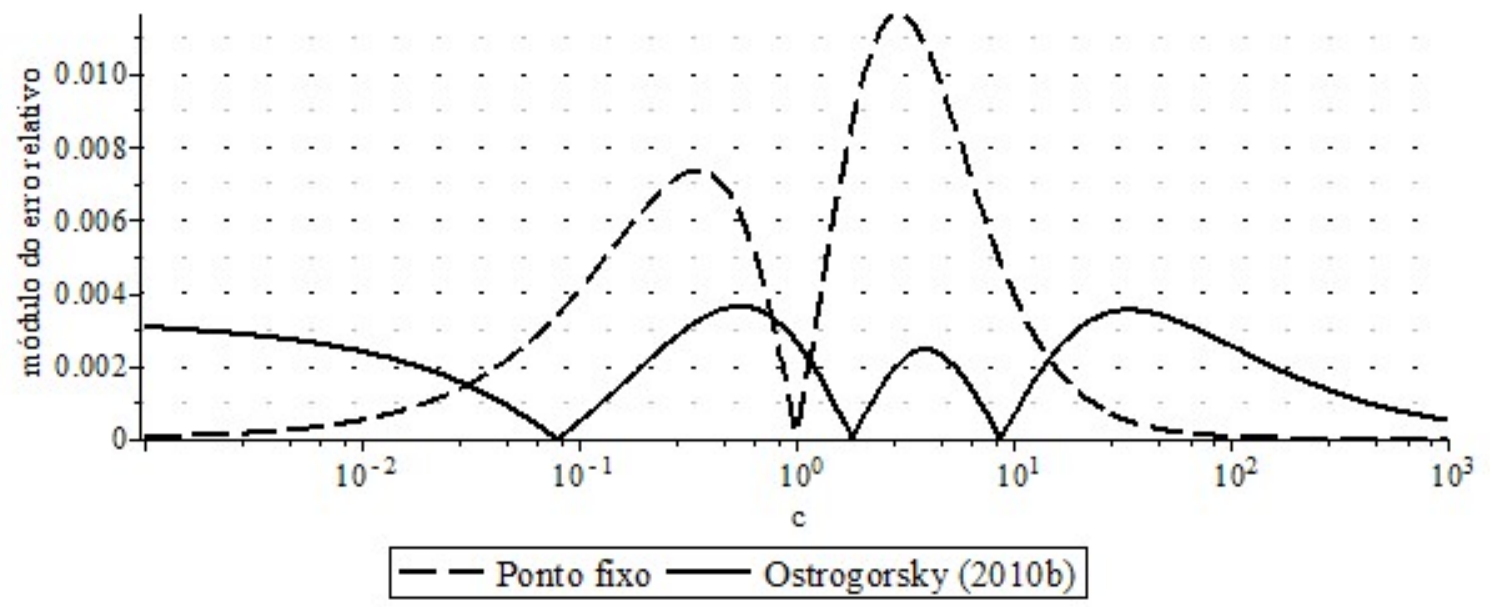

Figura 8.9 Expressões de intermediária exatidão para a primeira raiz da Eq. (1.7) 


\subsection{Expressões de Maior Exatidão para a Primeira Raiz}

A expressão de Beck et al. (1992) mostrada na Eq. (2.16):

$$
\beta_{1} \cong\left\{\begin{array}{l}
\sqrt{3\left(c-c^{2} / 5\right)}, 0 \leq c<0,4 \\
\frac{1}{2} \pi\left(1+\frac{3}{2(c+2)}\left(\sqrt{1+\frac{16(c-1)(c+2)}{3 \pi^{2}}}-1\right)\right), 0,4 \leq c<6 \\
\pi-\sqrt[3]{a+\frac{3 \pi}{2(c-1)}}+\sqrt[3]{a-\frac{3 \pi}{2(c-1)}}, c>6
\end{array}\right.
$$

para a primeira raiz da Eq. (1.7), onde

$$
a=\sqrt{\left(\frac{3 n \pi}{2(c-1)}\right)^{2}+\left(\frac{c}{c-1}\right)^{3}}
$$

é complexa demais para seu erro máximo de 0,9\%.

A expressão de Yovanovich (1996) mostrada na Eq. (2.19):

$$
\beta_{1} \cong \frac{\beta_{1, \infty}}{\left(1+\left(\frac{\beta_{1, \infty}}{\sqrt{m c}}\right)^{k}\right)^{\frac{1}{k}}}
$$

onde $k=2,139,2,238$ e 2,314 para a primeira raiz das Eqs. (1.5), (1.6) a (1.7), respectivamente, tem erros máximos de $0,22 \%$ a 0,7\%, bastante baixos em relação à sua simplicidade.

A expressão obtida a partir das expressões de outros autores, e que tem a mesma forma da expressão acima de Yovanovich (1996), mas que tem seus parâmetros recalculados através do conceito de MiniMax, mostrada na Eq. (5.4):

$$
\beta_{1} \cong\left((m c)^{\frac{r}{2}}+\beta_{1, \infty}^{r}\right)^{\frac{1}{r}}
$$

onde $r=-2,14,-2,25$ e - 2,34 para as Eqs. (1.5), (1.6) e (1.7), respectivamente, tem erros máximos de $0,20 \%$ a $0,50 \%$, valores estes menores do que os das expressões anteriores, e só não substitui a expressão de Ostrogorsky e Mikic (2008), que tem erros máximos de 2,5\% a 5\%, e a de ponto fixo, que tem erro máximo de 1,1\%, devido a suas simplicidades. 


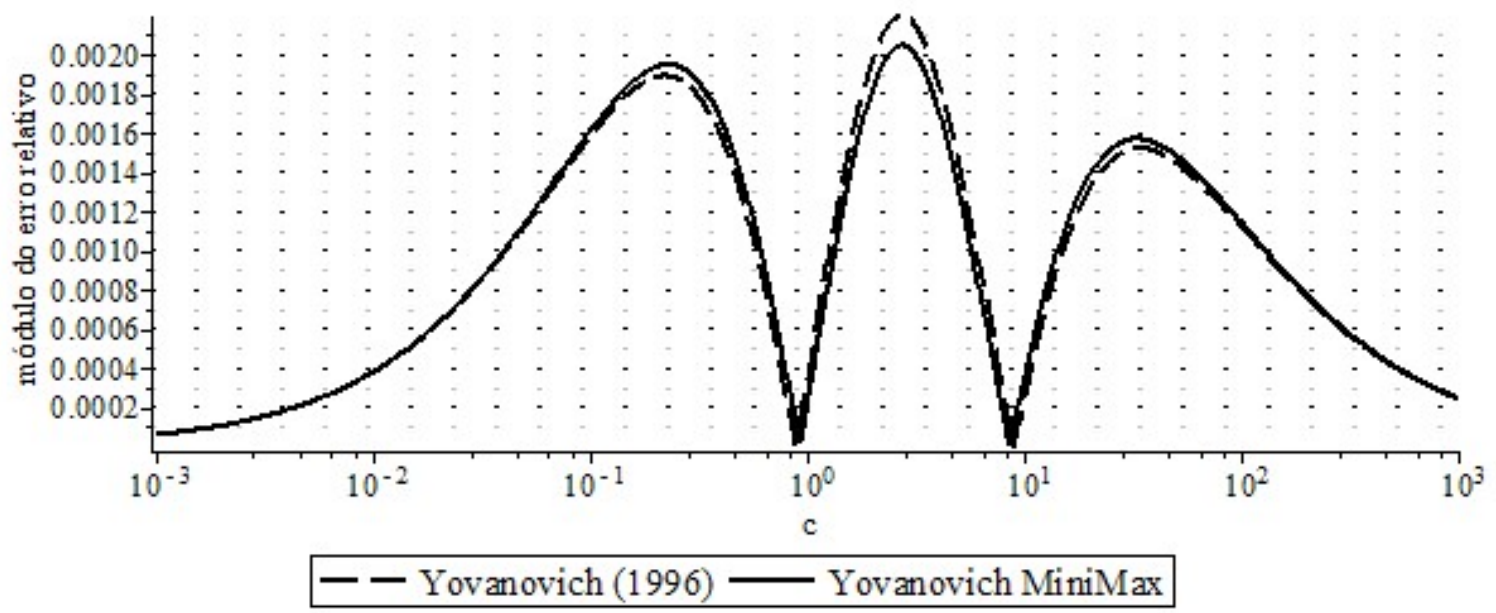

Figura 8.10 Expressões de maior exatidão para a primeira raiz da Eq. (1.5)

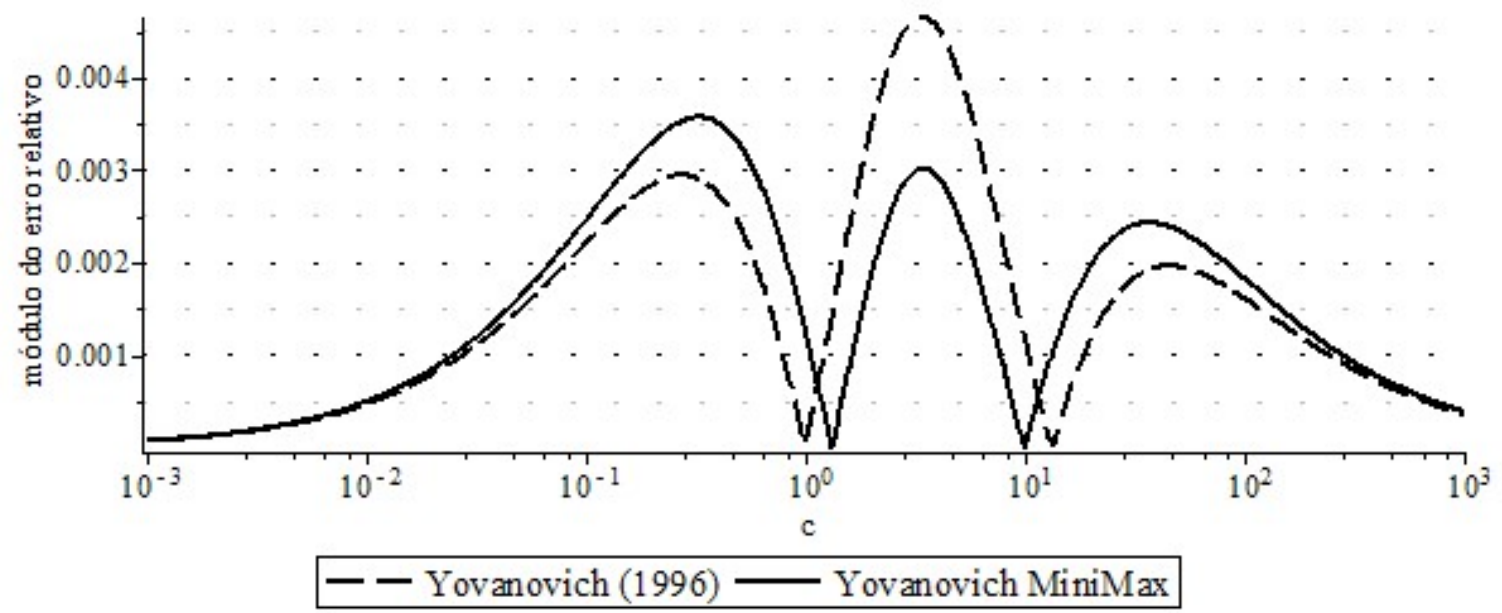

Figura 8.11 Expressões de maior exatidão para a primeira raiz da Eq. (1.7)

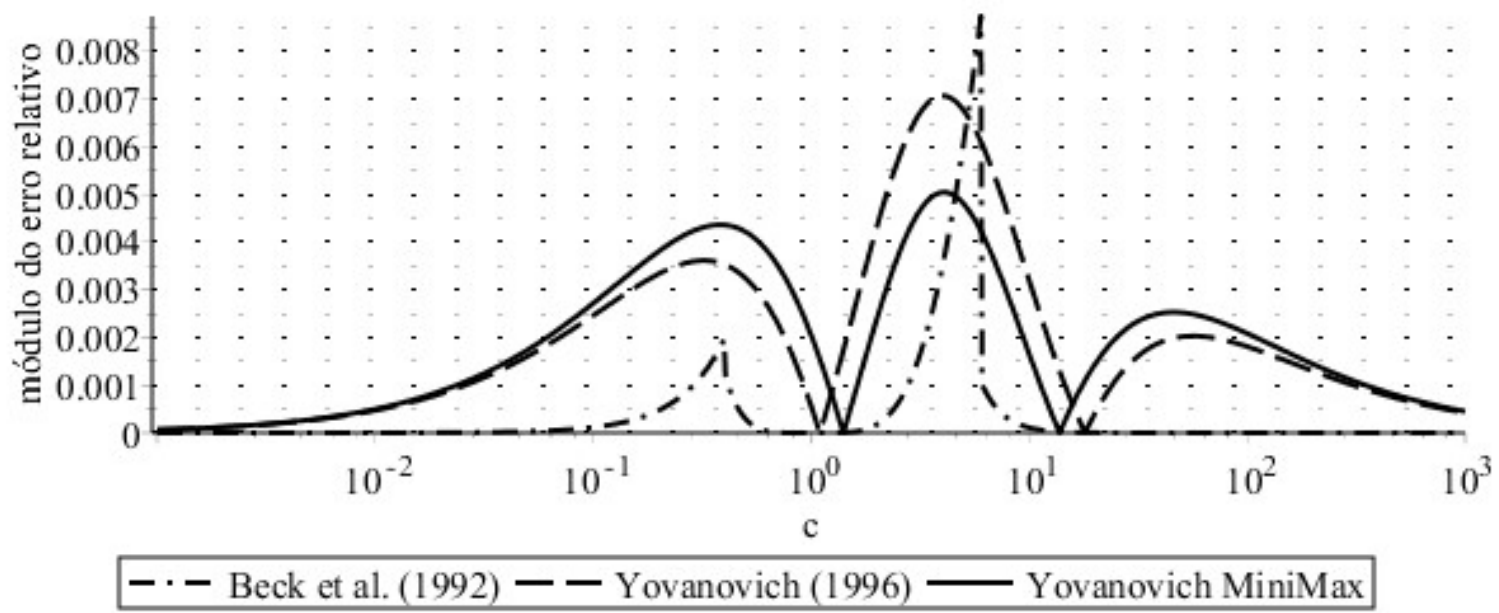

Figura 8.12 Expressões de maior exatidão para a primeira raiz da Eq. (1.7) 


\subsection{Expressões de Muito Maior Exatidão para a Primeira Raiz}

A expressão de Beck et al. (1992) mostrada na Eq. (2.15):

$$
\beta_{1} \cong\left\{\begin{array}{l}
\sqrt{\frac{3 c}{3+c}\left(1-\frac{1}{45}\left(\frac{3 c}{3+c}\right)^{2}\right)}, 0 \leq c \leq 2 \\
\frac{\pi c}{2(c+1)}\left(1+\frac{\pi^{2}}{12(c+1)^{3}+\pi^{2}(2 c-1)}\right), c>2
\end{array}\right.
$$

para a primeira raiz da Eq. (1.5), tem erro máximo de 0,08\%, mas tem muita complexidade além de não ter uma versão análoga para a Eq. (1.6),

As expressões obtidas por frações parciais mostradas nas Eqs. (6.8), (6.9) e (6.10):

$$
\begin{aligned}
& \beta_{1} \cong \sqrt{\frac{15(3 c+7)-\sqrt{15\left(107 c^{2}+350 c+735\right)}}{2(c+10)}} \\
& \beta_{1} \cong \sqrt{\frac{12(3 c+8)-4 \sqrt{3\left(19 c^{2}+48 c+192\right)}}{c+12}} \\
& \beta_{1} \cong \sqrt{\frac{105(c+3)-3 \sqrt{35\left(23 c^{2}+42 c+315\right)}}{2(c+14)}}
\end{aligned}
$$

respectivamente para a primeira raiz das Eqs. (1.5), (1.6) e (1.7), tem erros máximos de 0,028\% a 0,36\%, porém não podem ser agrupadas numa expressão apenas, sob pena de se ter muitos parâmetros de ajuste, e sua simplicidade reside no fato de ter sido construída apenas com números inteiros.

A expressão algébrica obtida no presente trabalho mostrada na (5.5):

$$
\beta_{1} \cong \frac{\sqrt{m A^{2} c+\beta_{1, \infty}^{2} c^{2 k}}}{A+c^{k}}
$$

onde $k=1,037,1,062,1,081$ e $A=4,025,5,63,7,25$ para a primeira raiz das Eqs. (1.5), (1.6) e (1.7) tem erros máximos de $0,066 \%$ a 0,22\%.

A expressão obtida a partir da aproximação de Padé de ordem [2, 1], mostrada na Eq. (6.11):

$$
\beta_{1} \cong a \sqrt{c+\frac{\beta_{1, \infty}^{2}}{m}-\sqrt{\left(c+\frac{\beta_{1, \infty}^{2}}{m}\right)^{2}-\frac{2 \beta_{1, \infty}^{2}}{a^{2}} c}}
$$

onde $a=1,6508,1,8483$ e 2,0215 para a primeira raiz das Eqs. (1.5), (1.6) e (1.7), tem erros de $0,033 \%$ a $0,16 \%$ e a consideramos a melhor entre as expressões de muito maior exatidão. 


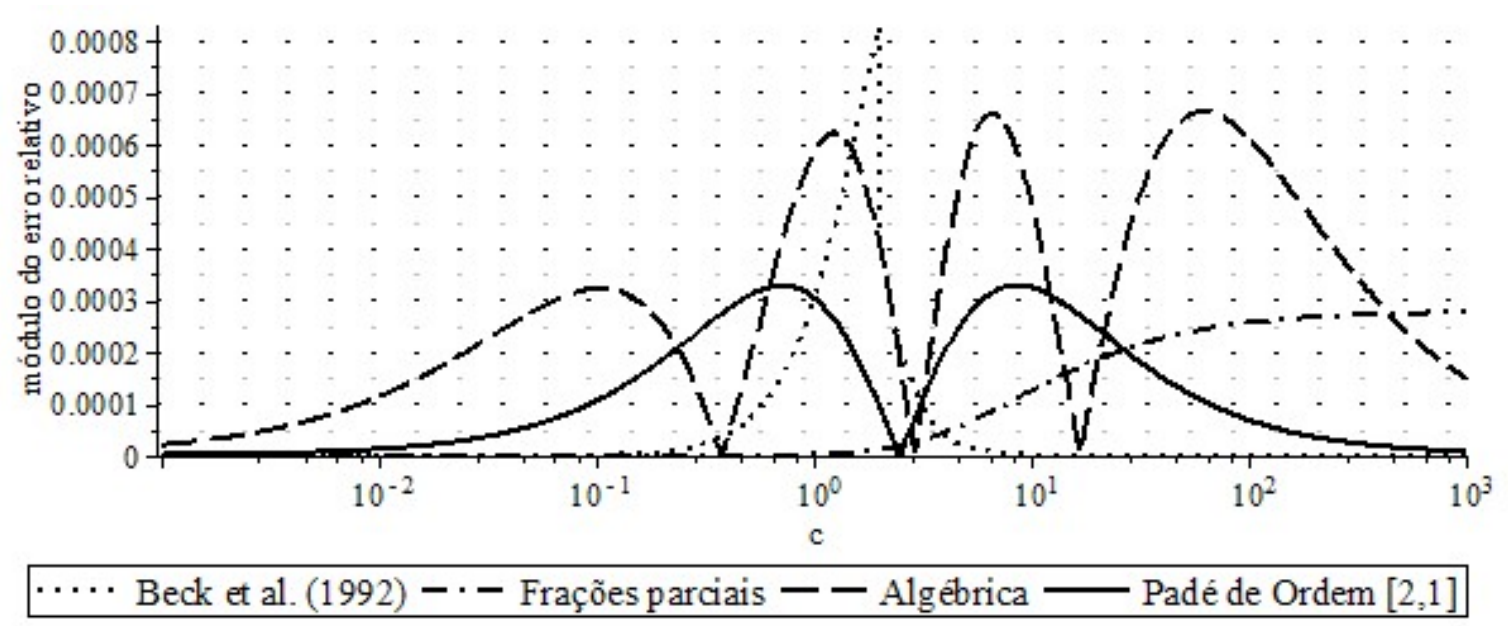

Figura 8.13 Expressões de muito maior exatidão para a primeira raiz da Eq. (1.5)

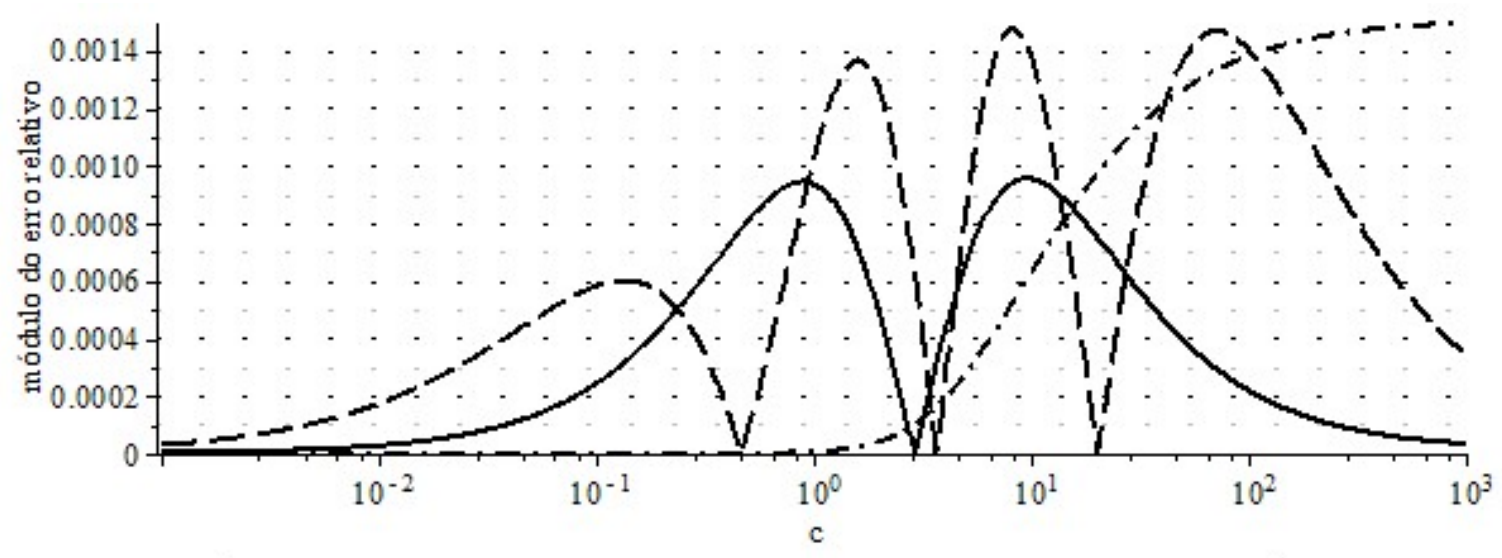

$-\cdot-$ Frações parciais -- Algébrica $\longrightarrow$ Padé de Ordem $[2,1]$

Figura 8.14 Expressões de muito maior exatidão para a primeira raiz da Eq. (1.7)

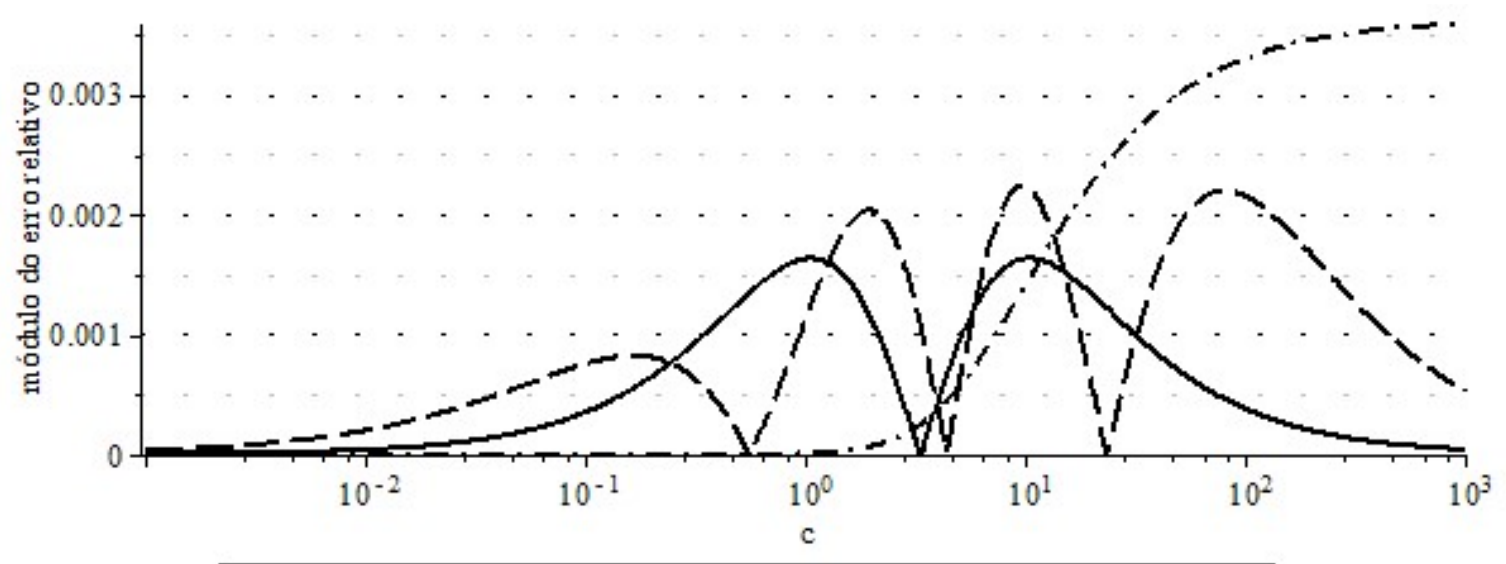

Frações parciais - - Algébrica — Padé de Ordem $[2,1]$

Figura 8.15 Expressões de muito maior exatidão para a primeira raiz da Eq. (1.7) 


\subsection{Uma Expressão de Extrema Exatidão para a Primeira Raiz}

A expressão obtida a partir da aproximação de Padé de ordem [2, 2], mostrada na Eq. (6.12):

$$
\beta_{1} \cong \sqrt{\frac{c+x_{1}-\sqrt{\left(1-\beta_{1, \infty}^{2} x_{2}\right)^{2} c^{2}+2 x_{1}\left(1-m x_{3}\right) c+x_{1}^{2}}}{x_{2} c+x_{3}}}
$$

onde $x_{1}=2,3250266, x_{2}=0,0468978$ e $x_{3}=0,449981$ para a Eq. (1.5), $x_{1}=2,636312, x_{2}=$ 0,030765 e $x_{3}=0,340892$ para a Eq. (1.6), e $x_{1}=2,937165, x_{2}=0,0220076$ e $x_{3}=0,274973$ para a Eq. (1.7), realmente não é simples, inclusive por possuir três parâmetros de ajuste, mas tem erros máximos de 0,000099\% a 0,0015\% os quais são, de longe, os menores até hoje obtidos (Figura 8.16 e Figura 8.17), e podem ser úteis em aplicações específicas.

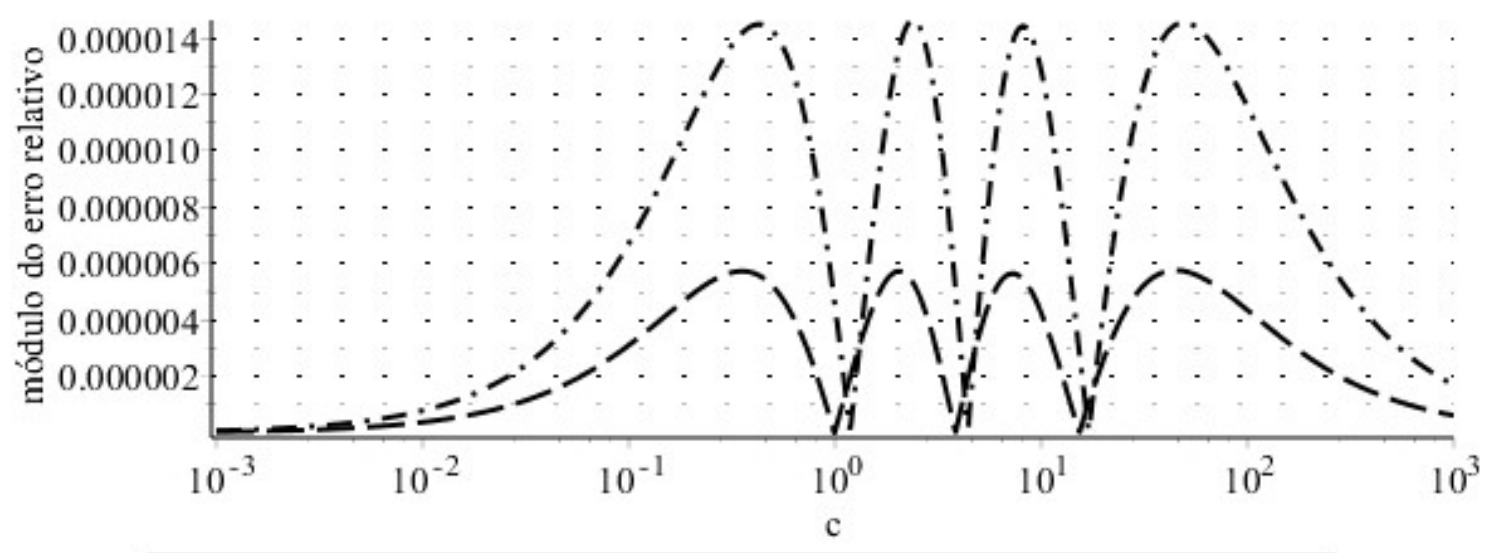

$-\cdot-$ Padé de Ordem [2,2] - Eq. (1.7) - - Padé de Ordem [2,2] - Eq. (1.6)

Figura 8.16 Expressão de extrema exatidão para a primeira raiz das Eqs. (1.5), (1.6) e (1.7)

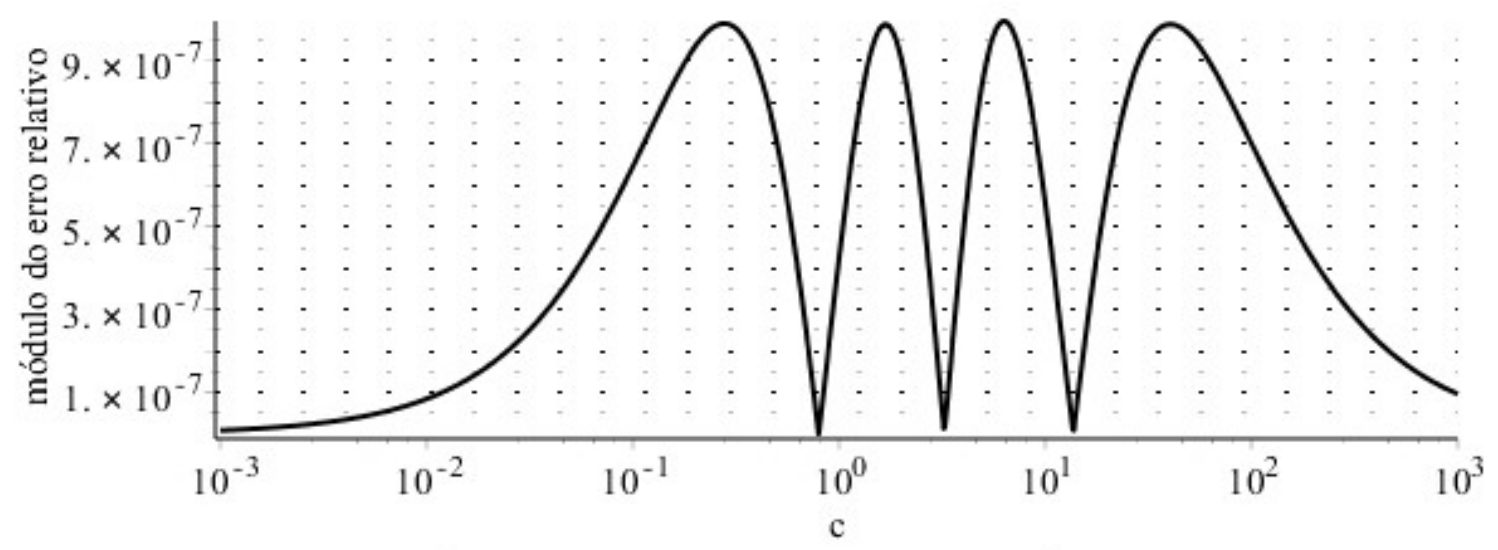

Padé de Ordem $[2,2]$ - Eq. (1.5)

Figura 8.17 Expressão de extrema exatidão para a primeira raiz das Eqs. (1.5), (1.6) e (1.7) 


\subsection{Expressões de Menor Exatidão para as Demais Raízes}

As expressões de Stevens e Luck (1999) mostradas nas Eqs. (2.31), (2.32) e (2.33), que podem ser reescritas como:

$$
\begin{aligned}
& \beta_{n} \cong\left(n-\frac{3}{4}\right) \pi+\frac{\pi}{4} \frac{c-\left(n-\frac{3}{4}\right) \pi}{c+\left(n-\frac{3}{4}\right) \pi} \\
& \beta_{n} \cong\left(n-\frac{1}{2}\right) \pi+\frac{\pi}{4} \frac{c-\left(n-\frac{1}{2}\right) \pi}{c+\left(n-\frac{1}{2}\right) \pi} \\
& \beta_{n} \cong\left(n-\frac{1}{2}\right) \pi+\frac{\pi}{2} \frac{c-\frac{2}{\pi}}{c+\left(n-\frac{1}{2}\right) \pi}
\end{aligned}
$$

para as demais raízes das Eqs. (1.5), (1.6) e (1.7), respectivamente, são bastante simples e, apesar de terem erros muito altos, de $1 \%$ a $3 \%$, elas são as únicas que são válidas para todo $n \geq 2$ da segunda equação.

As expressões de Beck et al. (1992) para as demais raízes da Eq. (1.5), mostradas nas Eq. (2.29):

$$
\beta_{n} \cong\left\{\begin{array}{l}
\frac{(n-1) \pi}{2(c+3)}\left(2 c+3+3 \sqrt{1+\frac{4 c(c+3)}{3(n-1)^{2} \pi^{2}}}\right), c \text { menor } \\
\frac{(2 n-1) \pi c}{2(c+1)}\left(1+\frac{(2 n-1)^{2} \pi^{2}}{12(c+1)^{3}+(2 n-1)^{2} \pi^{2}(2 c-1)}\right), c \text { maior }
\end{array}\right.
$$

onde a divisão deve ocorrer em $c=5,8,11$ e 13 para $n=2,3,4$ e 5, respectivamente, tem erro máximo de $0,23 \%$, mas são muito complexas e não valem para $n \geq 6$, enquanto as as expressões mostradas na Eq. (2.30):

$$
\beta_{n} \cong\left\{\begin{array}{l}
\left(n-\frac{1}{2}\right) \pi\left(1+\frac{3}{2(c+2)}\left(\sqrt{1+\frac{16(c-1)(c+2)}{3(2 n-1)^{2} \pi^{2}}}-1\right)\right), c<6 \\
\pi-\sqrt[3]{a+\frac{3 n \pi}{2(c-1)}}+\sqrt[3]{a-\frac{3 n \pi}{2(c-1)}}, c \geq 6
\end{array}\right.
$$

para as demais raízes da Eq. (1.7), onde

$$
a=\sqrt{\left(\frac{3 n \pi}{2(c-1)}\right)^{2}+\left(\frac{c}{c-1}\right)^{3}}
$$


além de serem muito complexas, tem erro que aumenta $\operatorname{com} n$, e não tem uma versão análoga para a Eq. (1.6),

A expressão de Haji-Sheik e Beck (2000) mostrada na Eq. (2.35):

$$
\beta_{n} \cong d_{n}+\frac{\pi}{2} \gamma_{n, 13} \xi_{13}
$$

para as demais raízes da Eq. (1.7), onde

$$
\begin{aligned}
\gamma_{n, 13}=1+\xi_{13}\left(1-\xi_{13}\right)\left(1-\frac{0.85}{n}+\right. & \left.\left(0.6-\frac{0.71}{n}\right)\left(1+\xi_{13}\right)\left(0.6+\frac{0.245}{n}-\xi_{13}\right)\right) \\
\xi_{13} & =\frac{c}{c+\pi d_{n} / 2} \\
d_{n} & =\left(n-\frac{1}{2}\right) \pi
\end{aligned}
$$

não só tem complexidade muito alta, como erro máximo de 4,9\%. Ela tem uma versão análoga para a Eq. (1.7) que será apresentada no próximo grupo de expressões de maior exatidão.

A expressão desenvolvida a partir da aproximação de Padé de ordem $[1,1]$ para as raízes da Eq. (1.5), mostrada na Eq. (7.1):

$$
\beta_{n} \cong \frac{2(n-1)(c+0,57)+\sqrt{c^{2}+c+4(n-1)^{2}}}{2 c / \pi+1}
$$

tem erro máximo de 0,56\% para a primeira raiz e de 0,59\% para as demais raízes. Ela apresenta a vantagem de ter equilíbrio entre a exatidão da primeira raiz e das demais raízes. Porém, não tem versões análogas para as Eqs. (1.6) e (1.7). 


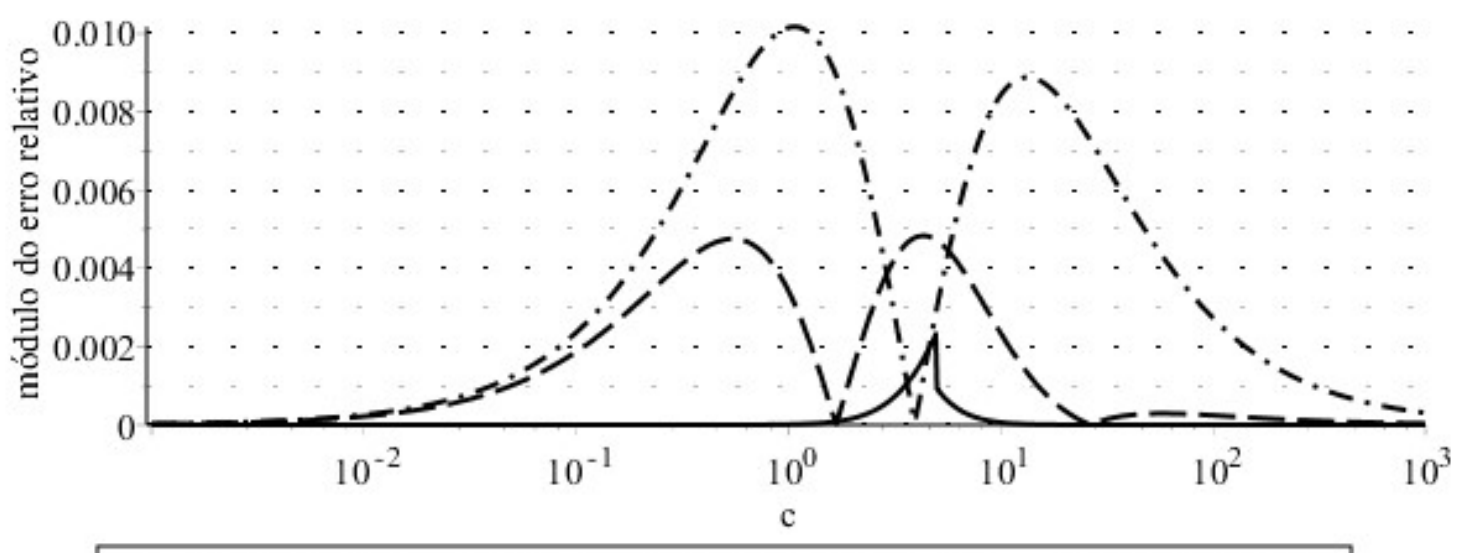

$-\cdot-$ Stevens e Luck (1999) $-\longrightarrow$ Padé de Ordem [1, 1] — Beck et al. (1992)

Figura 8.18 Expressões de menor exatidão para a segunda raiz da Eq. (1.5)

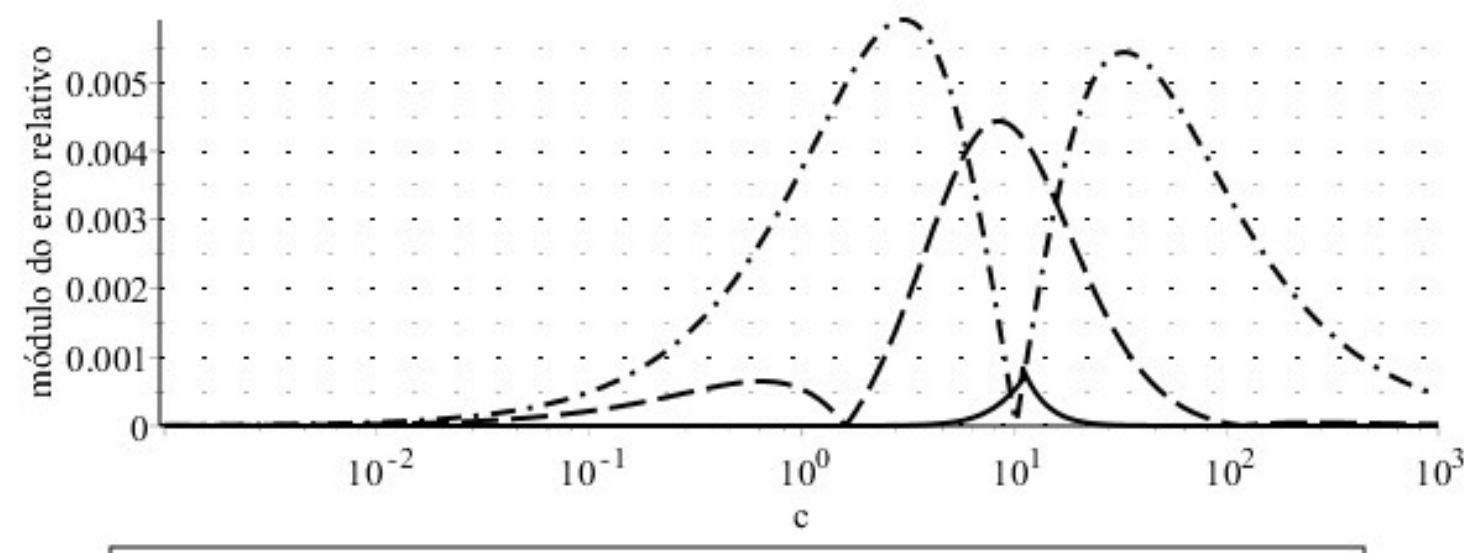

-*- Stevens e Luck (1999) $-\longrightarrow$ Padé de Ordem [1, 1] — Beck et al. (1992)

Figura 8.19 Expressões de menor exatidão para a quarta raiz da Eq. (1.5)

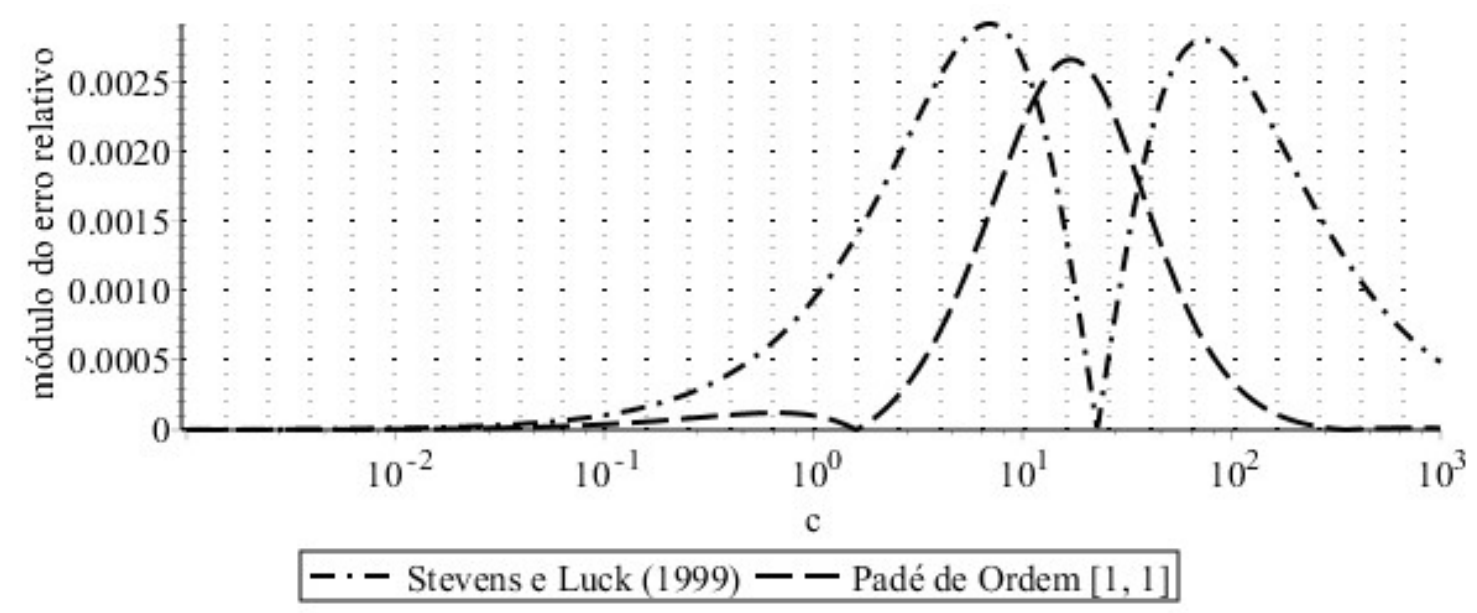

Figura 8.20 Expressões de menor exatidão para a oitava raiz da Eq. (1.5) 


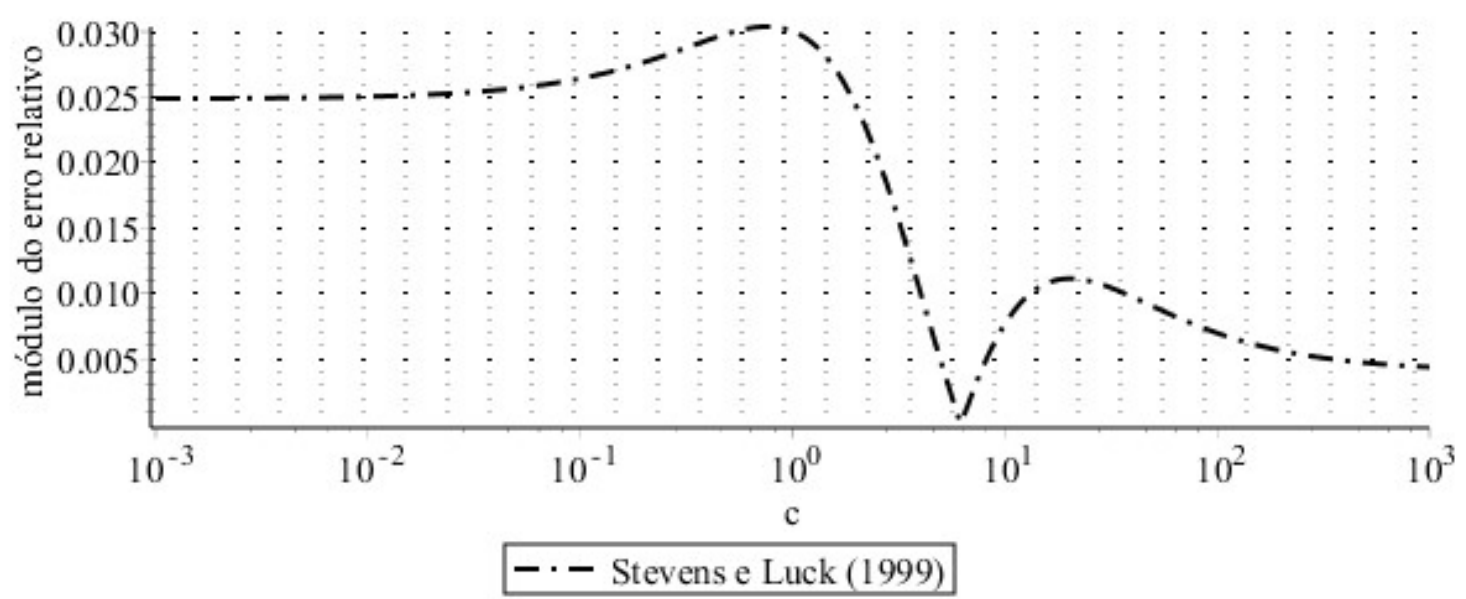

Figura 8.21 Expressões de menor exatidão para a segunda raiz da Eq. (1.6)

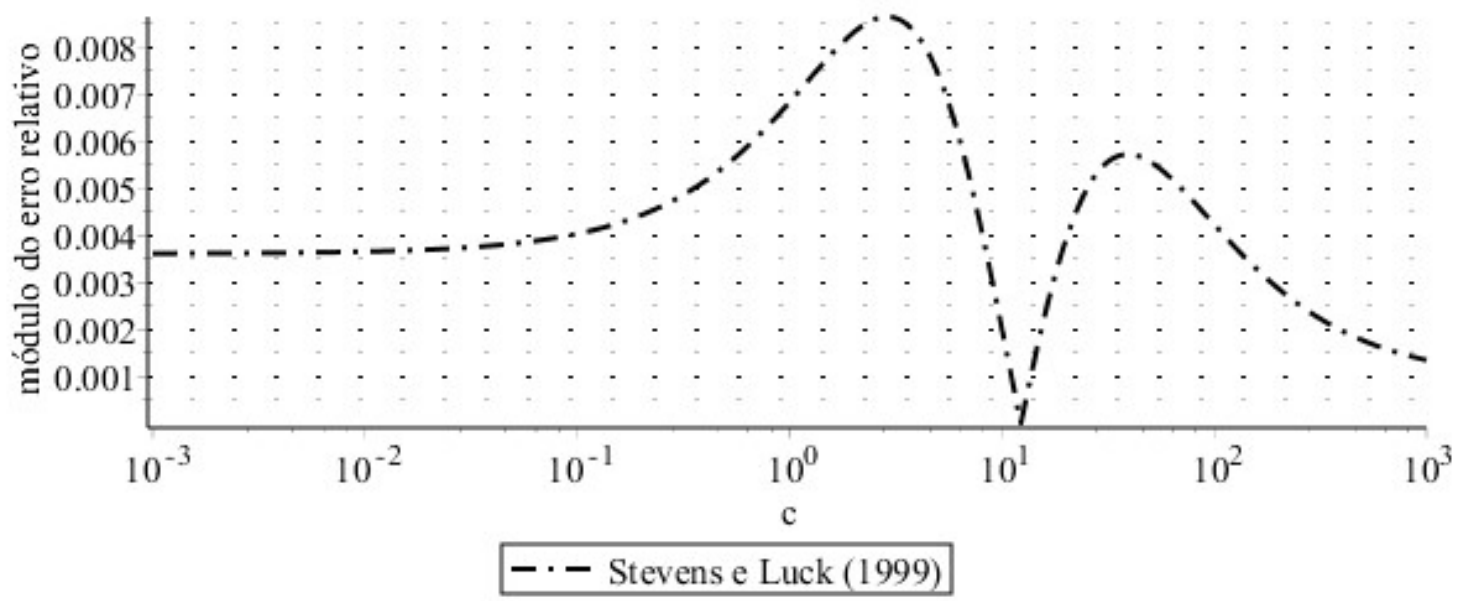

Figura 8.22 Expressões de menor exatidão para a segunda raiz da Eq. (1.6)

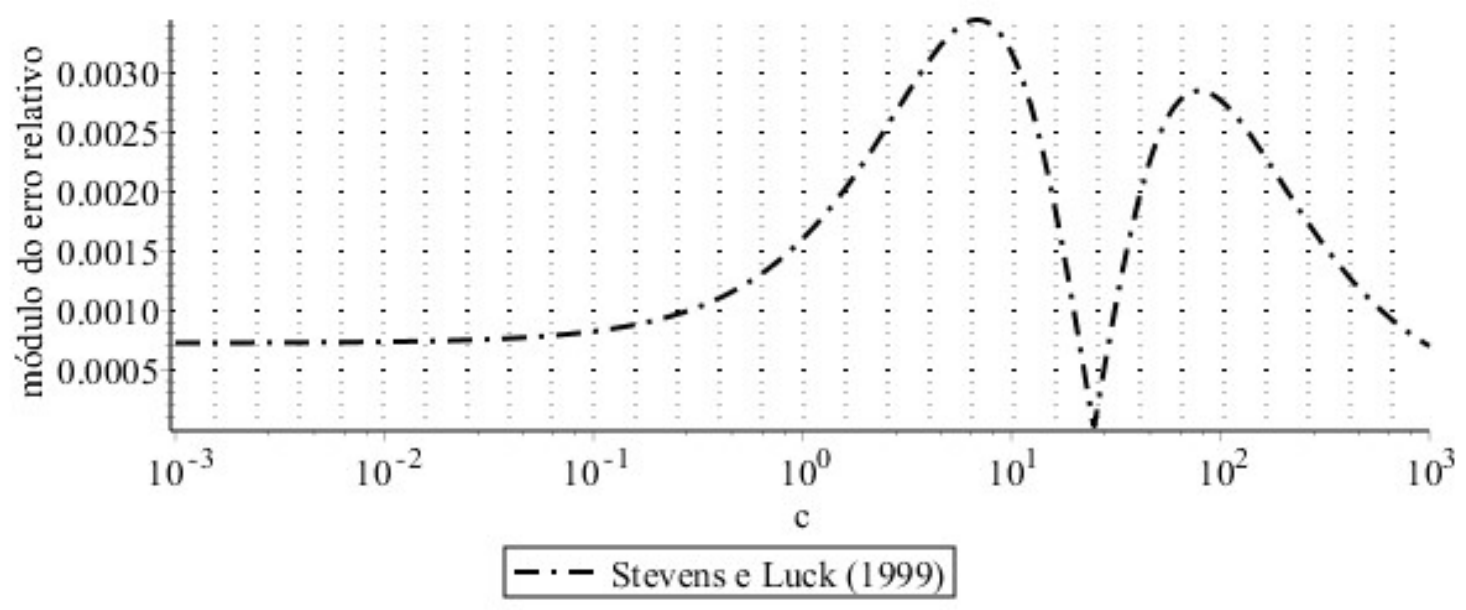

Figura 8.23 Expressões de menor exatidão para a oitava raiz da Eq. (1.6) 


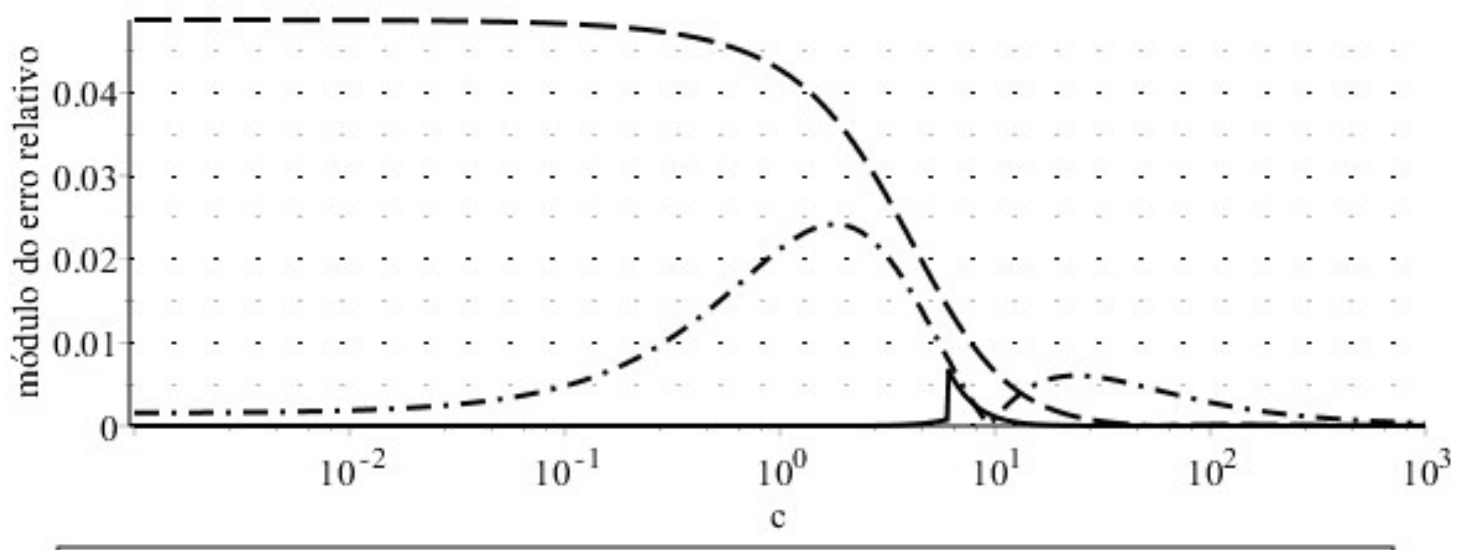

$-\cdot-$ Stevens e Luck (1999) - - Haji-Sheikh e Beck (2000) - Beck et al. (1992)

Figura 8.24 Expressões de menor exatidão para a segunda raiz da Eq. (1.7)

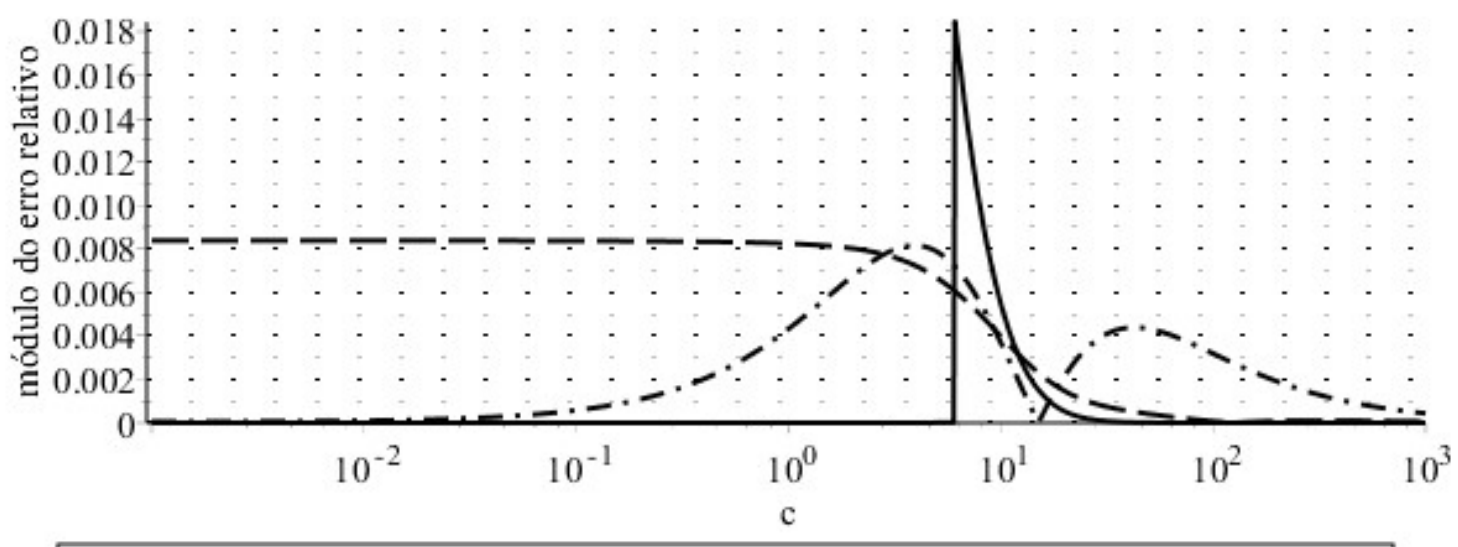

$-\cdot-$ Stevens e Luck (1999) - - Haji-Sheikh e Beck (2000) — Beck et al. (1992)

Figura 8.25 Expressões de menor exatidão para a quarta raiz da Eq. (1.7)

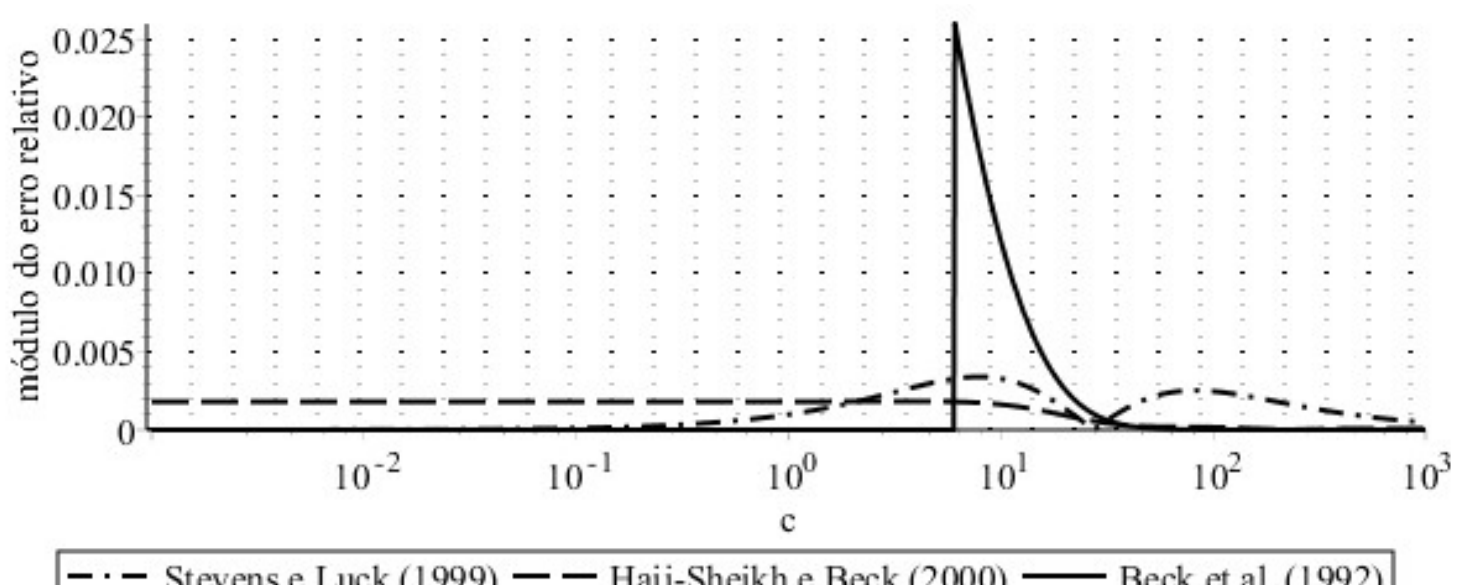

Figura 8.26 Expressões de menor exatidão para a oitava raiz da Eq. (1.7) 


\subsection{Expressões de Maior Exatidão para as Demais Raízes}

A expressão de Haji-Sheik e Beck (2000) mostrada na Eq. (2.34):

$$
\beta_{n} \cong c_{n}+\frac{\pi \gamma_{n, 23}}{4}\left(n^{-1} \xi_{23}+\left(1-n^{-1}\right) \frac{\tanh \xi_{23}}{\tanh 1}\right)
$$

para as demais raízes da Eq. (1.5), onde

$$
\begin{gathered}
\gamma_{n, 23}=1-1,04\left(\sqrt{\frac{c+c_{n}-\pi / 4}{c+H}}-\frac{c+\sqrt{H\left(c_{n}-\pi / 4\right)}}{c+H}\right) \\
\xi_{23}=\frac{c-c_{n}}{c+c_{n}} \\
H=0,76+1,22(n-1) \\
c_{n}=\left(n-\frac{3}{4}\right) \pi
\end{gathered}
$$

é muito complexa, além de não ter uma versão análoga para a Eq. (1.6).

A expressão de Yovanovich generalizada com parâmetros calculados de maneira a ser uma MiniMax, mostrada na Eq. (7.3):

$$
\beta_{n} \cong \beta_{n, 0}+\left(\left(\sqrt{m c+\left(\frac{m \beta_{n, 0}}{2}\right)^{2}}-\frac{m \beta_{n, 0}}{2}\right)^{r}+\left(\beta_{n, \infty}-\beta_{n, 0}\right)^{r}\right)^{\frac{1}{r}}
$$

para as Eqs. (1.5), (1.6) e (1.7), onde $r$ é dado na Tabela 7.2, não valem para $n \geq 10$, porém, elas tem a vantagem de manter a mesma forma com a primeira raiz (note-se que a tabela incluiu $n=1$ ).

A expressão algébrica mostrada na Eq. (7.4):

$$
\beta_{n} \cong \frac{\beta_{n, \infty} c^{k}+A \beta_{n, 0}}{c^{k}+A}
$$

para as demais raízes das Eqs. (1.5), (1.6) e (1.7), onde $k$ e $A$ são dados na Tabela 7.3, tem erro baixo para sua simplicidade, mas também não valem para $n \geq 10$.

As expressões obtidas a partir do ponto fixo mostradas nas Eqs. (7.5) e (7.6):

$$
\begin{gathered}
\beta_{n} \cong \arctan \frac{c}{\left(n-\frac{1}{2}\right) \pi-\frac{\pi}{2+\frac{2 c / \pi}{n-3 / 4}}}+(n-1) \pi \\
\beta_{n} \cong \operatorname{arccot} \frac{1-c}{\left(n-\frac{1}{2}\right) \pi-\frac{1-c \pi / 2}{(n-1 / 2) \pi+c}}+(n-1) \pi
\end{gathered}
$$


para as demais raízes da Eqs. (1.5) e (1.7), respectivamente, valem para qualquer $n$, inclusive $n=1$

(embora o erro não seja tão baixo nesse caso), são muito simples, e tem erro que se torna baixíssimo conforme $n$ aumenta, porém elas não tem uma versão para a Eq. (1.6). 


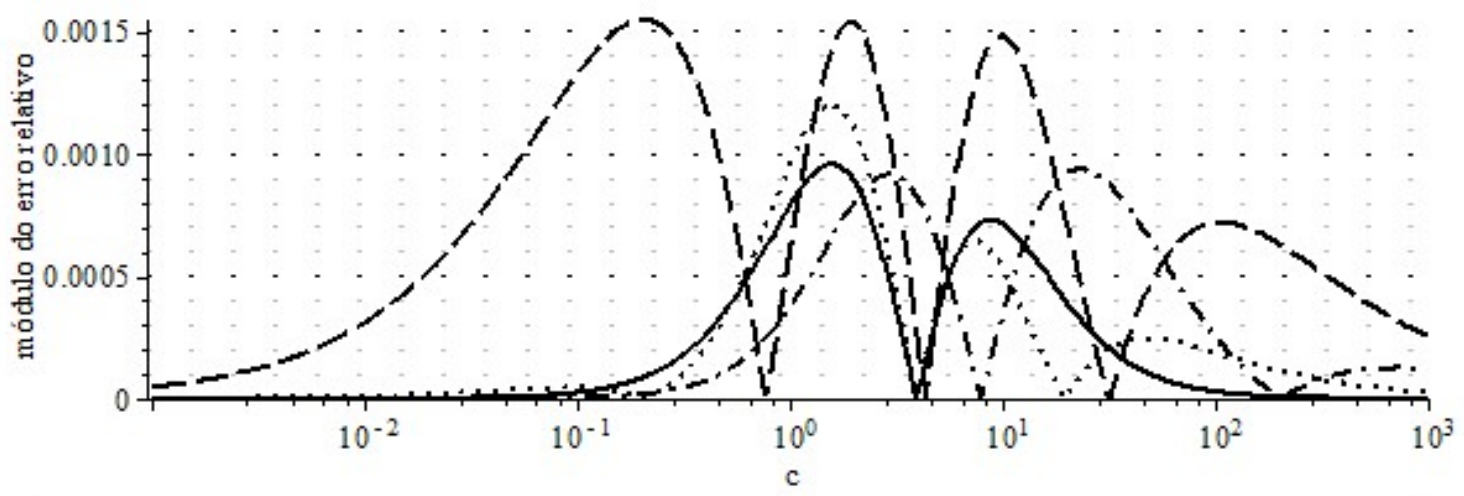
$\cdots \cdot$ Haji-Sheikh e Beck (2000) $-\cdot-$ Yovanovich minimax generalizada - Algébrica
Ponto fixo

Figura 8.27 Expressões de maior exatidão para a segunda raiz da Eq. (1.5)

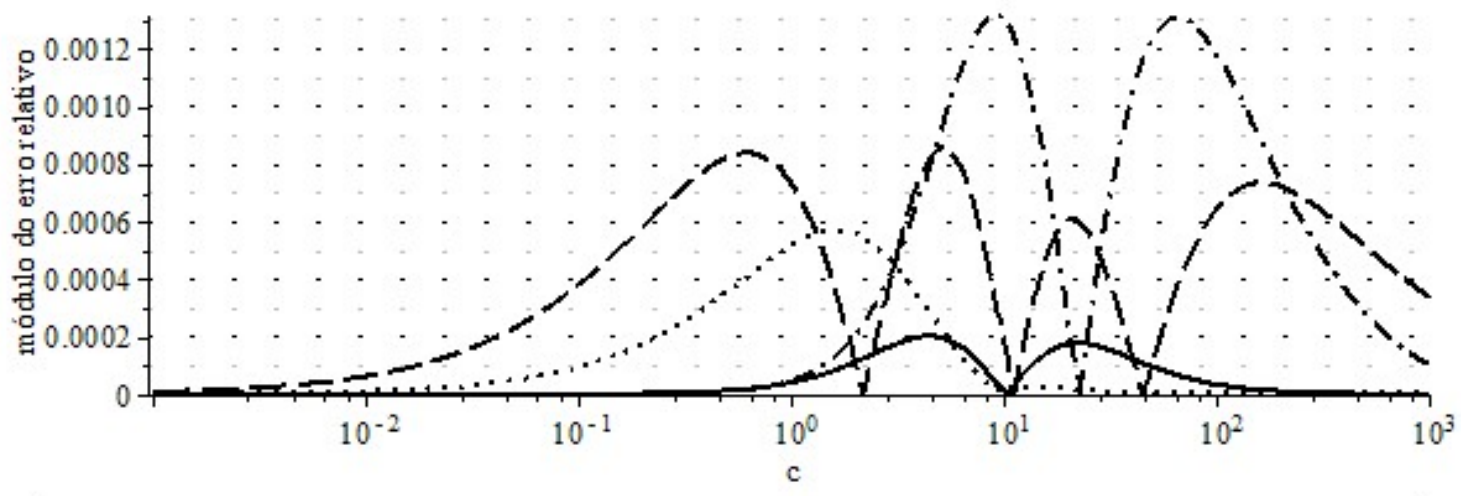

\section{Haji-Sheikh e Beck (2000) - - - Yovanovich minimax generalizada - - Algébrica} Ponto fixo

Figura 8.28 Expressões de maior exatidão para a quarta raiz da Eq. (1.5)

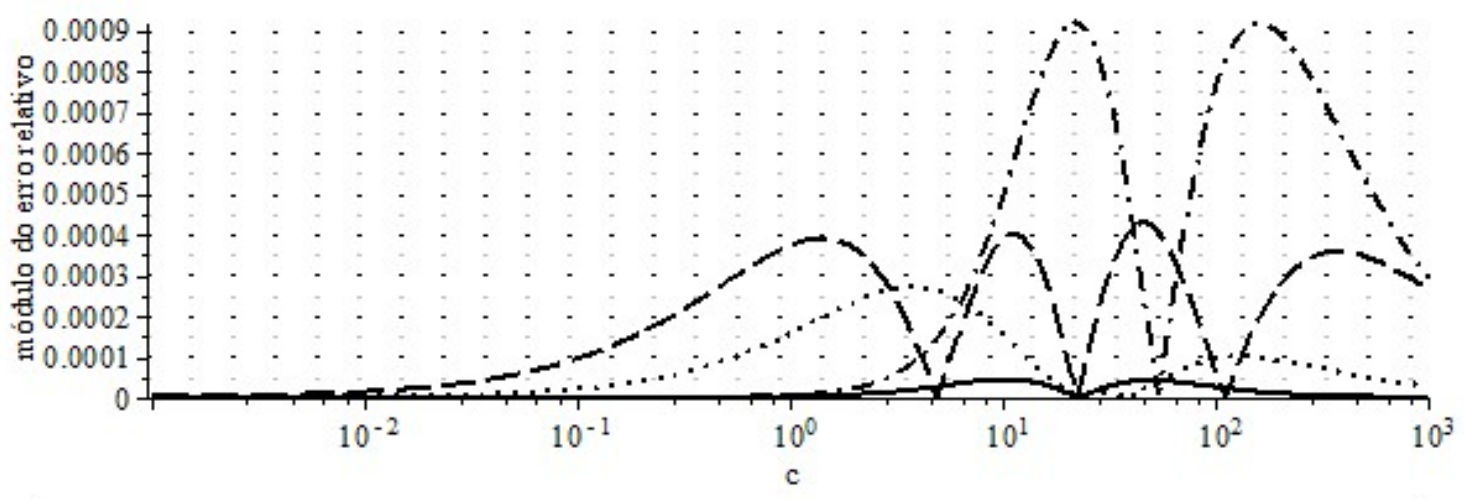

Haji-Sheikh e Beck (2000) - - - Yovanovich minimax generalizada - - Algébrica Ponto fixo

Figura 8.29 Expressões de maior exatidão para a oitava raiz da Eq. (1.5) 


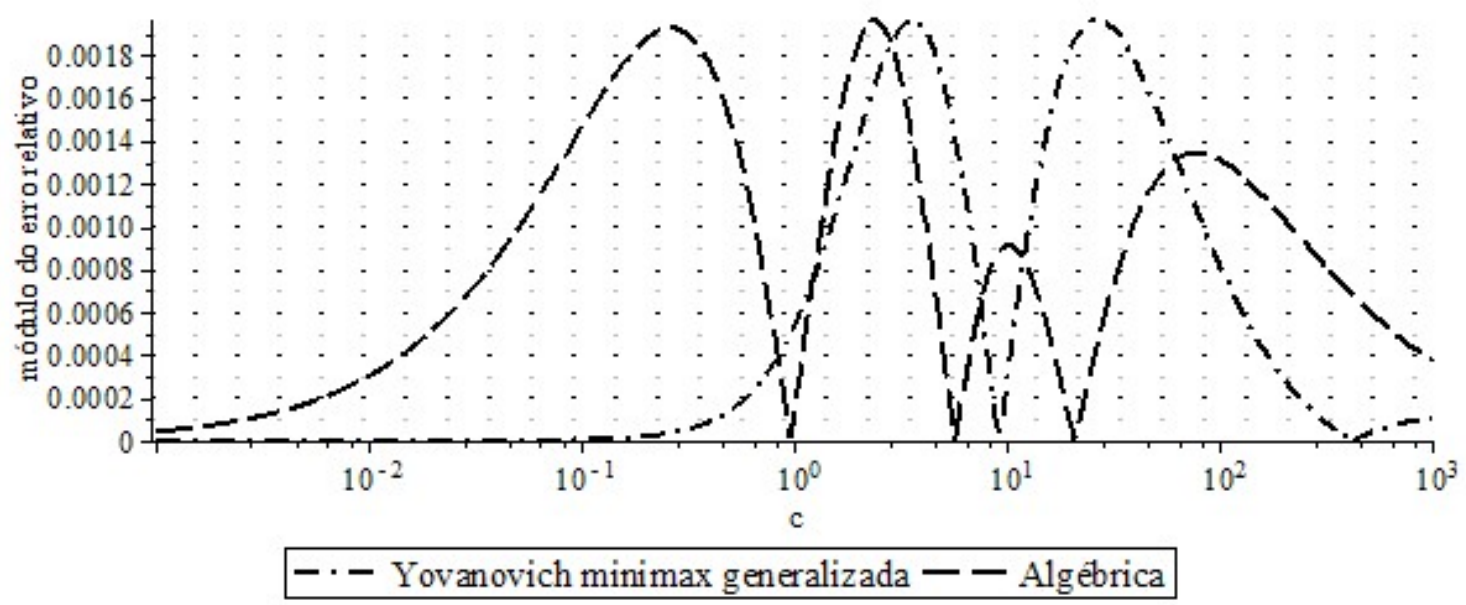

Figura 8.30 Expressões de maior exatidão para a segunda raiz da Eq. (1.6)

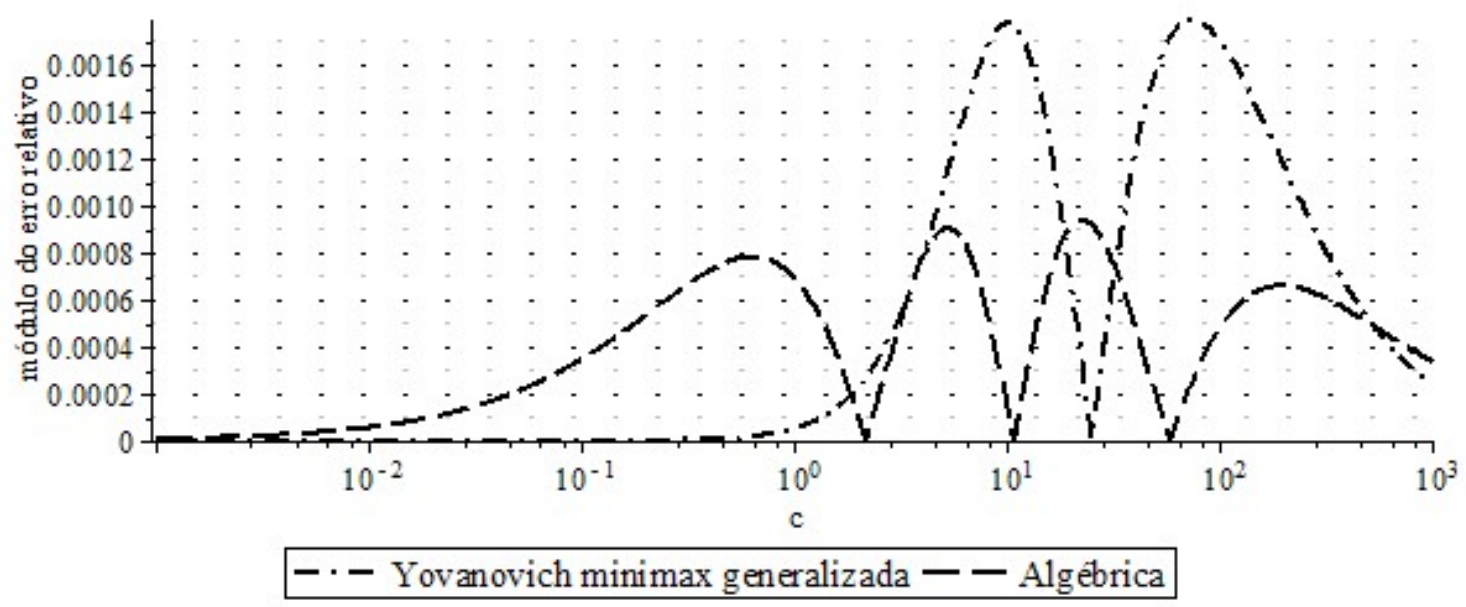

Figura 8.31 Expressões de maior exatidão para a quarta raiz da Eq. (1.6)

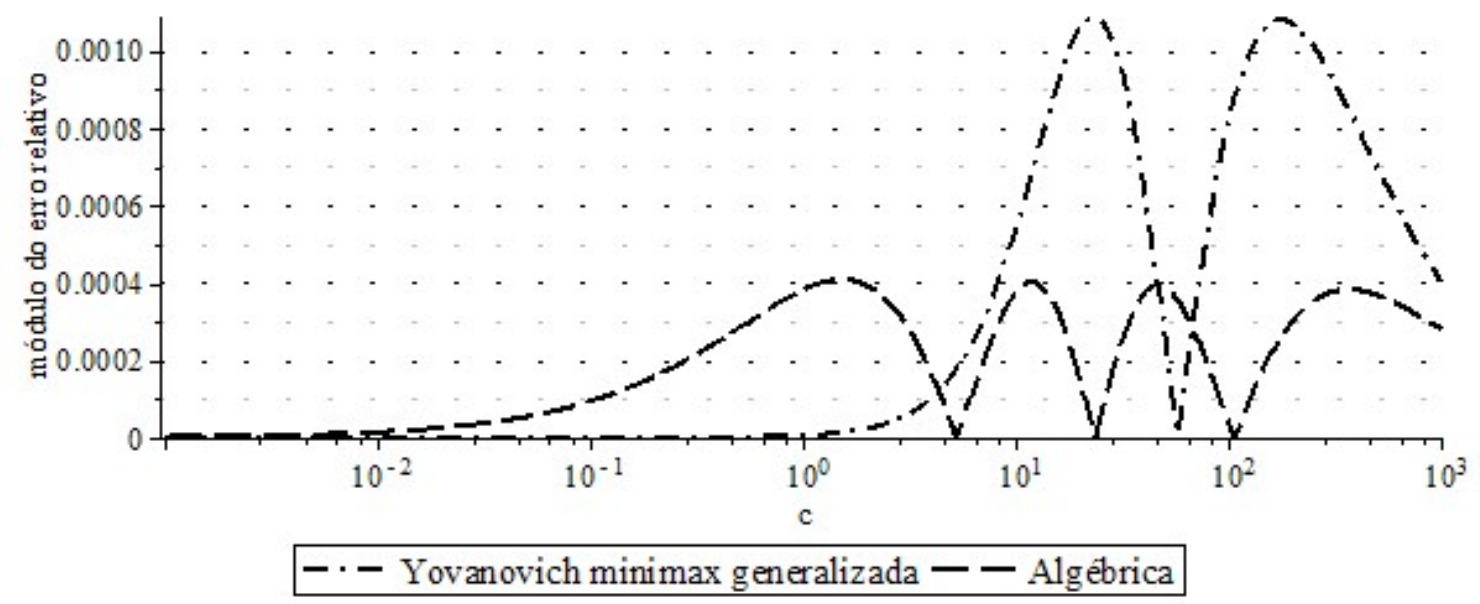

Figura 8.32 Expressões de maior exatidão para a oitava raiz da Eq. (1.6) 


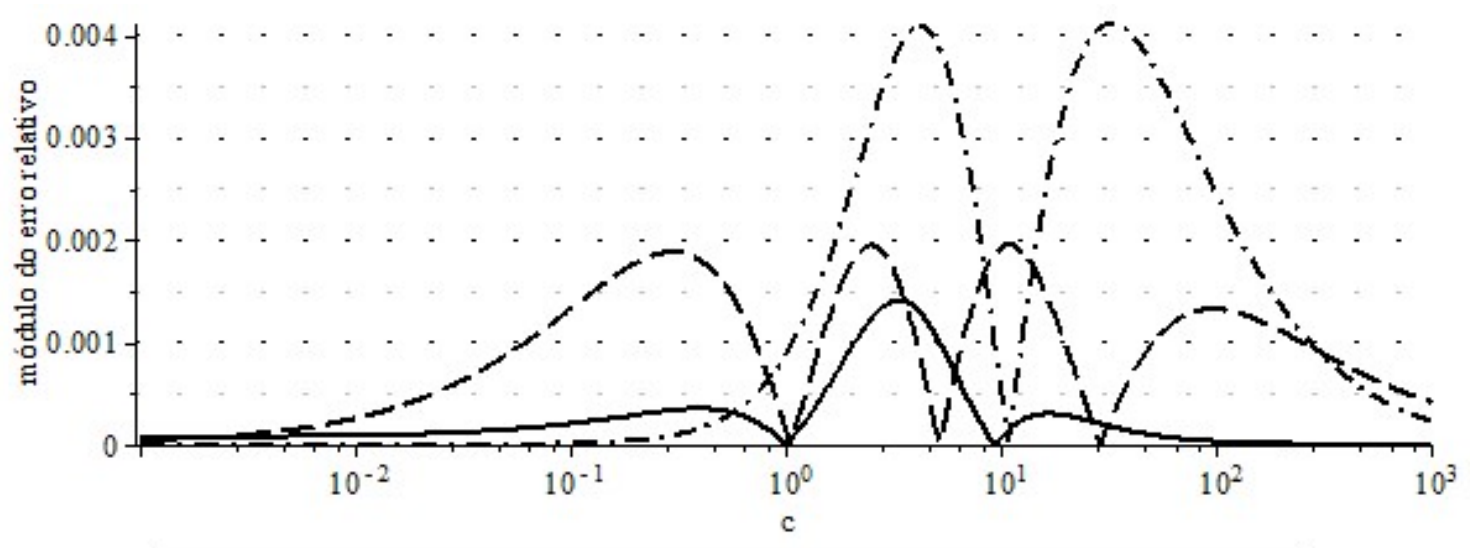

$-\cdot-$ Yovanovich minimax generalizada -- Algébrica - Ponto fixo

Figura 8.33 Expressões de maior exatidão para a segunda raiz da Eq. (1.7)

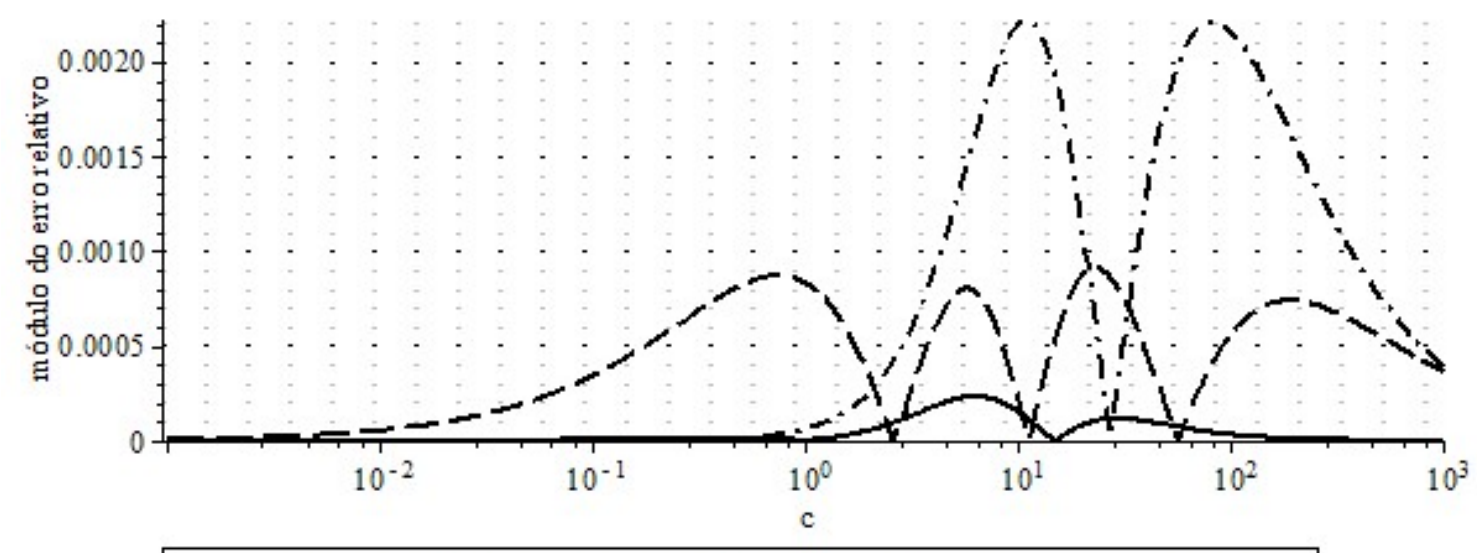

$-\cdot-$ Yovanovich minimax generalizada -- Algébrica $\longrightarrow$ Ponto fixo

Figura 8.34 Expressões de maior exatidão para a quarta raiz da Eq. (1.7)

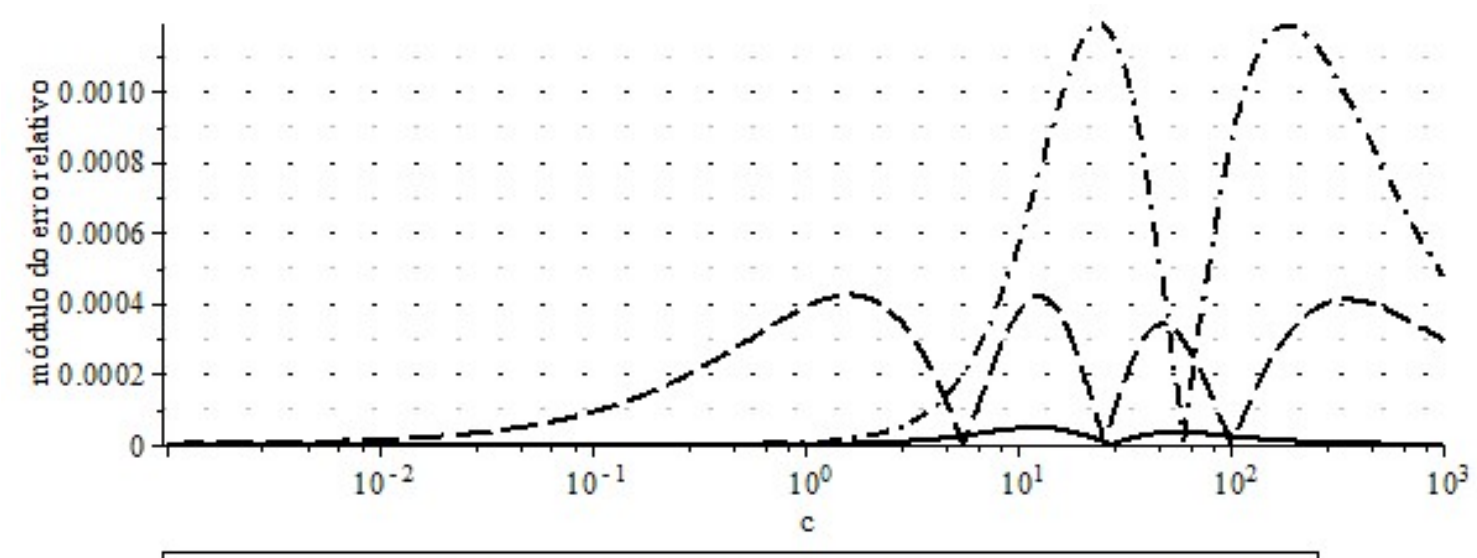

$-\cdot-$ Yovanovich minimax generalizada -- Algébrica - Ponto fixo

Figura 8.35 Expressões de maior exatidão para a oitava raiz da Eq. (1.7) 


\section{CONCLUSÕES E SUGESTÕES PARA PRÓXIMOS TRABALHOS}

Desenvolveu-se, neste trabalho, uma nova família de métodos iterativos para busca de raízes de equações, que gerou a expressão da Eq. (3.23), a qual, aplicada às raízes abordadas neste trabalho, que são as das Eqs. (1.5), (1.6) e (1.7), com valores inicias dados pelas Eqs. (3.35) e (3.36), e coeficientes dados pelas Eqs. (3.40) a (3.46), foi considerada como a que leva aos melhores resultados.

Não se desenvolveu expressões com maiores ordens que podem ser obtidas a partir dessa família de métodos, e um próximo trabalho poderá fazer isso. Estas, além das novas expressões que já foram desenvolvidas e aplicadas para as equações abordadas pelo presente trabalho, poderão ser aplicadas para outras equações.

Através do Teorema dos П de Buckingham, obteve-se uma compilação de funções iterativas clássicas, bem como das que foram desenvolvidas neste trabalho, mostradas na Tabela 3.1, a qual correlaciona as funções iterativas existentes e sugere outras novas. Próximos trabalhos poderão explorar essas novas funções.

Desenvolveu-se novas expressões aproximadas para a primeira e para as demais raízes das Eqs. (1.5), (1.6) e (1.7), e comparou-se suas exatidões com as das expressões já existentes. Com o desenvolvimento de novas expressões mais simples e com exatidão equivalente ou superior, algumas expressões antigas deixam de ter utilidade, sendo agora consideradas obsoletas. Deve-se lembrar que para cada aplicação existe uma determinada exatidão, e maiores exatidões são conseguidas com expressões menos simples.

Para a primeira raiz recomenda-se as seguintes expressões partindo das mais exatas (e mais complexas) até as mais simples (mas menos exatas):

1- Padé de ordem [2, 2];

2- Padé de ordem $[2,1]$;

3- Yovanovich MiniMax;

4- Ponto fixo (não contempla a Eq. (1.6));

5- Ostrogorsky e Mikic (2008).

Da mesma maneira, para o caso das demais raízes, recomenda-se utilizar as seguintes expressões (na mesma ordem já descrita):

1- Ponto fixo (não contempla a Eq. (1.6));

2- Algébrica ou Yovanovich MiniMax generalizada;

3- Padé de ordem [1, 1] (não contempla as Eqs. (1.5) e (1.6)); 
4- Stevens e Luck (1999).

Existe um equilíbrio entre a Algébrica e a Yovanovich MiniMax generalizada. Como a segunda raiz é mais importante do que as de ordens mais altas, a expressão de Yovanovich generalizada é melhor para o caso da Eq. (1.5), enquanto que a expressão algébrica é melhor para o caso da Eq. (1.7). No caso da Eq. (1.6), ambas apresentam exatidão próximas para a segunda raiz, mas para as raízes seguintes a expressão algébrica tem mais exatidão.

A expressão de Yovanovich MiniMax generalizada é mais simples do que a expressão algébrica porque contém apenas um parâmetro de ajuste, enquanto que a expressão algébrica possui dois parâmetros. Além disso, a expressão de Yovanovich generalizada mantém a mesma forma para a primeira raiz, enquanto que a expressão algébrica muda de forma.

A expressão obtida a partir da aproximação de Padé de ordem $[1,1]$ tem como vantagem o equilíbrio de exatidão entre a primeira e as demais raízes. Isto deve ser destacado aqui nas conclusões porque o estudo em separado das expressões para a primeira raiz dificulta se verificar este equilíbrio.

Comparando-se a exatidão dos valores que se obtém a partir de tabelas publicadas com as destas expressões recomendadas, nota-se que apenas a última expressão de cada uma dessas duas listas tem exatidão menor do que as que são obtidas a partir das tabelas.

O algoritmo de Remez está demonstrado na literatura para funções racionais, porém, verificou-se neste trabalho que ele leva a resultados corretos para outras funções, precisando ser adaptado em alguns casos. Um próximo trabalho conduzido por matemáticos poderá demonstrar este resultado aqui encontrado.

A metodologia desenvolvida para se obter expressões MiniMax através de gráficos e através da adaptação do algoritmo de Remez, poderá ser utilizada para se reajustar qualquer expressão de física ou engenharia que tenha utilizado o critério de mínimos quadrados com dados que não envolviam erros. Além disso, o modo pelo qual utilizamos a aproximação de Padé e as frações parciais para se desenvolver expressões de ajuste poderá ser utilizado para outras equações em próximos trabalhos. 


\section{Referências}

AMAT, S.; BUSQUIER, S.; GUTIÉRREZ, J. M. Geometric constructions of iterative functions to solve nonlinear equations. J. Comp. Appl. Math., v. 157, p. 197-205, 2003.

ARPACI, Vedat S. Conduction Heat Transfer. Reading, Massachusetts: Addison-Wesley, 1966.

BAKER Jr., G. A.; GRAVES-MORRIS, P. Padé approximants. $2^{\text {nd }}$ ed. Nova York: Cambridge University Press, 1996. p. 1-66.

BECK, J. V. et al. Heat Conduction Using Green's Functions. Washington, DC: Hemisphere, 1992. p. 487-496.

BURDEN, R. L.; FAIRES, D. J., BURDEN, A. M. Numerical Analysis. 10th ed. Boston, MA, USA: Cengage Learning, 2015. p. 74-75, ex. 5, 6.

BURNISTON, E. E.; SIEWERT, C. E. The use of Riemann problems in solving a class of transcedental equations. In: Mathematical Proceedings of the Cambridge Philosophical Society, v. 73, n. 1, Cambridge University Press, 1973, p. 111-118.

CARSLAW, H. S.; JAEGER, J. C. Conduction of Heat in Solids. 2nd ed. London:Oxford, 1959.

CHURCHILL, S. W.; USAGI, R. A General Expression for the Correlation of Rates of Transfer and Other Phenomena. AIChE J., v. 18, p. 1121-1132, 1972.

ClAESSENS, G.; LOIZOU, G.; WUYTACK, L. Comments on a root finding method using Padé approximation. BIT, v. 17, p. 360-361, 1977.

CRANK, J. The Mathematics of Diffusion. 2nd ed. New York: Oxford University Press, 1975.

FIELD, D. A. Convergence rates for Padé-based iterative solutions of equations. J. Comp. Appl. Math., v. 32, p. 69-75, 1990. 
FRANKEL, S. Complete Approximate Solutions of the Equation $\mathrm{x}=\tan \mathrm{x}$. National Mathematics Magazine, v. 11, n. 4, p. 177-182, 1937.

GONNET, Gaston H. A Study of Iteration Formulas for Root Finding, Where Mathematics, Computer Algebra and Software Engineering Meet. In: European Congress of Mathematics. v. 202. Barcelona, 2001, p. 143-156.

HAHN, D. W.; ÖZIŞIK, M. N. Heat Conduction. 3rd ed. Hoboken: Wiley, 2012.

HAJI-SHEIKH, A.; BECK, J. V. An Efficient method of computing eigenvalues in heat conduction. Numerical Heat Transfer, Part B, v. 38, p. 133-156, 2000.

HOUSEHOLDER, A. S. The Numerical Treatment of a Single Nonlinear Equation. New York: McGraw-Hill, 1970, p. 140 a 179.

KANWAR, V; SINGH, S; Bakshi, S. Simple geometric constructions of quadratically and cubically convergent iterative functions to solve nonlinear equations. Numer. Algorithms, v. 47, p. 95-107, 2008.

KUNG, H. T.; TRAUB, J. F. Optimal order of one-poin and multipoint iteration. J. ACM, v. 21 n. 4, p. 643$651,1974$.

LEATHERS, R. A.; MCCORMICK, N. J. Closed-form solutions for transcendental equations of heat transfer. Journal of Heat Transfer, v. 118, p. 970-973, 1996.

LI, S. et al. Halley's iterative formula based on Padé approximation and its modifications. J. Inform. Comput. Sci., v. 9, n. 4, p. 997-1004, 2012.

LORENTZEN, L. Padé aprroximation and continued fractions. Applied Numerical Mathematics, v. 60, p. 1364-1370, 2010.

LUCK, R.; STEVENS, J. W. Explicit Solutions for Transcendental Equations. SIAM Review, v. 44, n. 2, p. 227-233, 2002. 
LUIKOV, A. V. Analytical Heat Diffusion Theory. New York: Academic Press, 1968, p. 294-295.

MERZ, G. Padésche Näherungsbrüche und Iterationsverfahren höherer Ordnung. Computing, v. 3, p. 165-183, 1968.

MIKHAILOV, M. D.; ÖZIŞIK, M. N. On the determination of the roots of some classes of transcendental equations. Comunications in Applied Numerical Methods, v. 2 p. 437-446, 1986.

MORSE, P. M.; FESHBACK, H. Methods of Theoretical Physics. New York: McGraw-Hill, 1953.

NETA, B.; SCOTT, M.; CHUN, C. Basins of attraction for several methods to find simple roots of nonlinear equations, Appl. Math. Comput., v. 218, p. 10548-10556, 2012.

NOUREIN, M. Root determination by use of Padé approximants. BIT, v. 16, p. 291-297, 1976.

OSTROGORSKY, A. G. Simple explicit equations for transient heat conduction in finite solids. Journal of Heat Transfer, v. 131, p. 011303-1 - 011303-11, 2009.

OSTROGORSKY, A. G.; MIKIC, B. B. Explicit equations for transient heat conduction in finite solids for $\mathrm{Bi}>$ 2. Heat and Mass Transfer, v. 45, p. 375-380, 2009.

OSTROGORSKY, A. G.; MIKIC, B. B. Explicit solutions for boundary value problems in diffusion of heat and mass, Journal of Crystal Growth, v. 310, p. 2691-2696, 2008.

PAKDEMIRLI, M.; BOYACIC, H. Generation of root finding algorithms via perturbation theory and some formulas, Applied Mathematics and Computation, v. 184, p. 783-788, 2007.

PETKOVIĆ, M.; HERCEG, D. On rediscovered iteration methods for solving equations. J. Comp. Appl. Math., v. 107, p. 275-284, 1999.

PETKOVIĆ, M. S.; PETKOVIĆ, L. D.; HERCEG, Đ. On Schröder's families of root-finding methods. J. Comp. Appl. Math., v. 233, p. 1755-1762, 2010.

POWELL, M. J. D. Approximation Theory and Methods. Cambridge: University Press, 1981. 
SAKURAI, T.; TORII, T.; SUGIURA, H. An iterative method for algebraic equation by Padé approximation. Computing, v. 46, n. 2, p. 131-141, 1991.

STEVENS, J. W.; LUCK, R. Explicit approximations for all eigenvalues of the 1-D transient heat conduction equations. Heat Transfer Engineering, v. 20, n. 2, p. 35-41, 1999.

STOVER, C.; Weisstein, E. W. Closed-Form Solution. MathWorld - A Wolfram Web Resource. Disponível em: < http://mathworld.wolfram.com/Closed-FormSolution.html>. Acesso em: 20 nov. 2014.

TRAUB, J. F. Iterative methods for the solution of equations. Englewood Cliffs, NJ, USA: PrenticeHall, 1964, p. 5.0-1 a 5.5-10.

YOVANOVICH, M. M. Simple Explicit Expressions for Calculation of the Heisler-Grober Charts. In: 1996 ASME National Heat Transfer Conference. Thermophysics and Thermophysical Properties Session. AIAA Paper 96-3968, Houston, TX, Aug. 3-6, 1996, p. 1-9.

ZHIZHCHENKO, A. B. Algebraic Function. In: Encyclopedia of Mathematics. 2011. Disponível em: $<$ http://www.encyclopediaofmath.org/index.php?title=Algebraic_function\&oldid=16758>. Acesso em: 20 nov. 2014.

ZUCKER, R. Elementary Transcendental Functions. In: M. Abramowitz, I.A. Stegun (Eds.). Hand-book of Mathematical Functions with Formulas, Graphs, and Mathematical Tables. National Bureau of Standards AMS 55. New York: Dover Publications, 1964. p. 224-225. 


\section{APÊNDICE A - O Método da Separação de Variáveis}

0 problema de valor de contorno:

$$
\left\{\begin{array}{l}
\frac{\partial^{2} T}{\partial x^{2}}+\frac{\partial^{2} T}{\partial y^{2}}=0, \quad 0<x<L, 0<y<W \\
T(0, y)=0, \quad 0<y<W \\
\frac{\partial T}{\partial x}(L, y)+H T(L, y)=0, \quad 0<y<W \\
T(x, 0)=0, \quad 0<x<L \\
T(x, W)=f(x), \quad 0<x<L
\end{array}\right.
$$

pode ser resolvido pelo método da separação de variáveis. Substituindo:

$$
T(x, y)=\sum_{n}\left(X_{n}(x) Y_{n}(y)\right)
$$

obtém-se:

$$
\left\{\begin{array}{l}
\frac{\partial^{2}}{\partial x^{2}} \sum_{n}\left(X_{n}(x) Y_{n}(y)\right)+\frac{\partial^{2}}{\partial y^{2}} \sum_{n}\left(X_{n}(x) Y_{n}(y)\right)=0 \\
\left.\sum_{n}\left(X_{n}(x) Y_{n}(y)\right)\right|_{x=0}=0 \\
\left.\frac{\partial}{\partial x} \sum_{n}\left(X_{n}(x) Y_{n}(y)\right)\right|_{x=L}+\left.H \sum_{n}\left(X_{n}(x) Y_{n}(y)\right)\right|_{x=L}=0 \\
\left.\sum_{n}\left(X_{n}(x) Y_{n}(y)\right)\right|_{y=0}=0 \\
\left.\sum_{n}\left(X_{n}(x) Y_{n}(y)\right)\right|_{y=W}=f(x)
\end{array}\right.
$$

que pode ser simplificada para:

$$
\left\{\begin{array}{l}
\sum_{n}\left(X_{n}^{\prime \prime}(x) Y_{n}(y)+X_{n}(x) Y_{n}^{\prime \prime}(y)\right)=0 \\
\sum_{n}\left(X_{n}(0) Y_{n}(y)\right)=0 \\
\sum_{n}\left(X_{n}^{\prime}(L) Y_{n}(y)+H X_{n}(L) Y_{n}(y)\right)=0 \\
\sum_{n}\left(X_{n}(x) Y_{n}(0)\right)=0 \\
\sum_{n}\left(X_{n}(x) Y_{n}(W)\right)=f(x)
\end{array}\right.
$$

Uma das maneiras para que as somas acima sejam nulas é fazer todos termos nulos, então: 


$$
\left\{\begin{array}{l}
X_{n}^{\prime \prime}(x) Y_{n}(y)+X_{n}(x) Y_{n}^{\prime \prime}(y)=0 \\
X_{n}(0) Y_{n}(y)=0 \\
\left(X_{n}^{\prime}(L)+H X_{n}(L)\right) Y_{n}(y)=0 \quad, \text { para todo } n \\
X_{n}(x) Y_{n}(0)=0 \\
\sum_{n}\left(X_{n}(x) Y_{n}(W)\right)=f(x)
\end{array}\right.
$$

Rearranjando a primeira equação, e observando que uma das maneiras para que as três equações seguintes sejam nulas é fazer os termos constantes nulos, obtém-se:

$$
\left\{\begin{array}{l}
\frac{X_{n}^{\prime \prime}(x)}{X_{n}(x)}=-\frac{Y_{n}^{\prime \prime}(y)}{Y_{n}(y)} \\
X_{n}(0)=0 \\
X_{n}^{\prime}(L)+H X_{n}(L)=0 \quad, \text { para todo } n \\
Y_{n}(0)=0 \\
\sum_{n}\left(X_{n}(x) Y_{n}(W)\right)=f(x)
\end{array}\right.
$$

Como ambos lados da primeira equação dependem de variáveis diferentes, a única maneira da igualdade ser satisfeita é fazê-las constantes, então:

$$
\frac{X_{n}^{\prime \prime}(x)}{X_{n}(x)}=-\frac{Y_{n}^{\prime \prime}(y)}{Y_{n}(y)}=-\lambda_{n}
$$

Isso permite desacoplar o problema $\operatorname{com} X$ :

$$
\left\{\begin{array}{l}
\left\{\begin{array}{l}
X_{n}^{\prime \prime}(x)+\lambda_{n} X_{n}(x)=0 \\
X_{n}(0)=0 \\
X_{n}^{\prime}(L)+H X_{n}(L)=0
\end{array}\right. \\
\left\{\begin{array}{l}
Y_{n}^{\prime \prime}(y)-\lambda_{n} Y_{n}(y)=0 \\
Y_{n}(0)=0 \\
\sum_{n}\left(X_{n}(x) Y_{n}(W)\right)=f(x)
\end{array}, \text { para todo } n\right.
\end{array}\right.
$$

Se $\lambda_{n}<0$, então:

$$
\begin{gathered}
X_{n}(x)=A_{n} \operatorname{senh} \sqrt{-\lambda_{n}} x+B_{n} \cosh \sqrt{-\lambda_{n}} x \\
X_{n}(0)=0 \rightarrow B_{n}=0 \rightarrow X_{n}(x)=A_{n} \operatorname{senh} \sqrt{-\lambda_{n}} x \\
X_{n}^{\prime}(L)+H X_{n}(L)=0 \rightarrow A_{n} \sqrt{-\lambda_{n}} \cosh \sqrt{-\lambda_{n}} L+H A_{n} \operatorname{senh} \sqrt{-\lambda_{n}} L=0 \\
\rightarrow A_{n}\left(\sqrt{-\lambda_{n}} \cosh \sqrt{-\lambda_{n}} L+H \operatorname{senh} \sqrt{-\lambda_{n}} L\right)=0 \rightarrow A_{n}=0 \rightarrow X_{n}(x)=0
\end{gathered}
$$

já que estas funções hiperbólicas são estritamente positivas para argumentos positivos.

Se $\lambda_{n}=0$, então:

$$
\begin{gathered}
X_{n}(x)=A_{n} x+B_{n} \\
X_{n}(0)=0 \rightarrow B_{n}=0 \rightarrow X_{n}(x)=A_{n} \\
X_{n}^{\prime}(L)+H X_{n}(L)=0 \rightarrow A_{n}=0 \rightarrow X_{n}(x)=0
\end{gathered}
$$

Se $\lambda_{n}>0$, então: 


$$
\begin{gathered}
X_{n}(x)=A_{n} \operatorname{sen} \sqrt{\lambda_{n}} x+B_{n} \cos \sqrt{\lambda_{n}} x \\
X_{n}(0)=0 \rightarrow B_{n}=0 \rightarrow X_{n}(x)=A_{n} \operatorname{sen} \sqrt{\lambda_{n}} x \\
X_{n}^{\prime}(L)+H X_{n}(L)=0 \rightarrow A_{n}\left(\sqrt{\lambda_{n}} \cos \sqrt{\lambda_{n}} L+H \operatorname{sen} \sqrt{\lambda_{n}} L\right)=0 \\
\rightarrow A_{n}=0 \text { ou } \sqrt{\lambda_{n}} \cos \sqrt{\lambda_{n}} L+H \operatorname{sen} \sqrt{\lambda_{n}} L=0 \\
A_{n}=0 \rightarrow X_{n}(x)=0 \\
\sqrt{\lambda_{n}} \cos \sqrt{\lambda_{n}} L+H \operatorname{sen} \sqrt{\lambda_{n}} L=0 \rightarrow \\
\zeta_{n} \cos \zeta_{n} L+H \operatorname{sen} \zeta_{n} L=0
\end{gathered}
$$

onde $\zeta_{n}=\sqrt{\lambda_{n}}$.

Como $X_{n}(x)=0$ leva a $T(x, y)=0$, a qual não é solução do problema inicial, a única condição acima válida é $\lambda_{n}>0$. Neste caso:

$$
\begin{gathered}
Y_{n}^{\prime \prime}(y)-\lambda_{n} Y_{n}(y)=0 \rightarrow Y_{n}(y)=C_{n} \operatorname{senh} \zeta_{n} y+D_{n} \cosh \zeta_{n} y \\
Y_{n}(0)=0 \rightarrow D_{n}=0 \rightarrow Y_{n}(y)=C_{n} \operatorname{senh} \zeta_{n} y
\end{gathered}
$$

Portanto:

$$
T(x, y)=\sum_{n}\left(X_{n}(x) Y_{n}(y)\right)=\sum_{n}\left(A_{n} \operatorname{sen} \zeta_{n} x C_{n} \operatorname{senh} \zeta_{n} y\right)=\sum_{n}\left(E_{n} \operatorname{sen} \zeta_{n} x \operatorname{senh} \zeta_{n} y\right)
$$

Resta obter $E_{n}$ a partir da última condição de contorno:

$$
T(x, W)=\sum_{n}\left(E_{n} \operatorname{sen} \zeta_{n} x \operatorname{senh} \zeta_{n} W\right)=f(x)
$$

0 valor de $T(x, W)$ para $x<0$ é irrelevante, então pode-se considerar que $f(x)$ é uma função ímpar e a sua expansão por série de Fourier é simplesmente:

$$
f(x)=\frac{a_{0}}{2}+\sum_{n=1}^{\infty}\left(a_{n} \cos \zeta_{n} x+b_{n} \operatorname{sen} \zeta_{n} x\right)=\sum_{n=1}^{\infty}\left(b_{n} \operatorname{sen} \zeta_{n} x\right)
$$

já que

$$
a_{n}=\frac{1}{L} \int_{-L}^{L} f(x) \cos \zeta_{n} x d x=0
$$

e o produto da função ímpar $f(x)$ pela função par $\cos \zeta_{n} x$ resulta numa função impar que tem integral nula neste caso de intervalo simétrico em relação a origem $[-L, L]$.

Portanto:

$$
\begin{gathered}
E_{n} \operatorname{senh} \zeta_{n} W=b_{n}=\frac{1}{L} \int_{-L}^{L} f(x) \operatorname{sen} \zeta_{n} x d x \\
\rightarrow E_{n}=\frac{1}{L \operatorname{senh} \zeta_{n} W} \int_{-L}^{L} f(x) \operatorname{sen} \zeta_{n} x d x
\end{gathered}
$$


Observe-se que se fosse utilizado $X_{n}(x)=0$ para alguns $n$, não se teria uma base completa para a expansão de $f(x)$. Observe-se também como o $T(x, y)$ obtido satisfaz todas equações do problema de valor de contorno:

$$
\begin{gathered}
\frac{\partial^{2} T}{\partial x^{2}}+\frac{\partial^{2} T}{\partial y^{2}}=\frac{\partial^{2}}{\partial x^{2}} \sum_{n}\left(E_{n} \operatorname{sen} \zeta_{n} x \operatorname{senh} \zeta_{n} y\right)+\frac{\partial^{2}}{\partial y^{2}} \sum_{n}\left(E_{n} \operatorname{sen} \zeta_{n} x \operatorname{senh} \zeta_{n} y\right)= \\
\sum_{n}\left(E_{n} \frac{\partial^{2}}{\partial x^{2}} \operatorname{sen} \zeta_{n} x \operatorname{senh} \frac{n \pi y}{L}+E_{n} \operatorname{sen} \zeta_{n} x \frac{\partial^{2}}{\partial y^{2}} \operatorname{senh} \zeta_{n} y\right)= \\
\sum_{n}\left(E_{n}\left(-\zeta_{n}^{2}\right) \operatorname{sen} \zeta_{n} x \operatorname{senh} \zeta_{n} y+E_{n} \operatorname{sen} \zeta_{n} x\left(\zeta_{n}^{2}\right) \operatorname{senh} \zeta_{n} y\right)=0 \\
T(0, y)=\sum_{n}\left(E_{n} \operatorname{sen} \zeta_{n} 0 \operatorname{senh} \zeta_{n} y\right)=0 \\
T(L, y)=\sum_{n}\left(E_{n} \operatorname{sen} \zeta_{n} L \operatorname{senh} \zeta_{n} y\right)=0 \\
T(x, 0)=\sum_{n}\left(E_{n} \operatorname{sen} \zeta_{n} x \operatorname{senh} \zeta_{n} 0\right)=0 \\
T(x, W)=\sum_{n}\left(E_{n} \operatorname{sen} \zeta_{n} x \operatorname{senh} \beta_{n} W\right)=\sum_{n=1}^{\infty}\left(b_{n} \operatorname{sen} \zeta_{n} x\right)=f(x)
\end{gathered}
$$

Se uma das paredes que está com temperatura fixada em $0^{\circ} \mathrm{C}$, estivesse, em vez disso, isolada, o problema seria:

$$
\left\{\begin{array}{l}
\frac{\partial^{2} T}{\partial x^{2}}+\frac{\partial^{2} T}{\partial y^{2}}=0, \quad 0<x<L, 0<y<W \\
\frac{\partial T}{\partial x}(0, y)=0, \quad 0<y<W \\
\frac{\partial T}{\partial x}(L, y)+H T(L, y)=0, \quad 0<y<W \\
T(x, 0)=0, \quad 0<x<L \\
T(x, W)=f(x), \quad 0<x<L
\end{array}\right.
$$

e a mudança ocorreria em:

$$
\left\{\begin{array}{l}
X_{n}^{\prime \prime}(x)+\lambda_{n} X_{n}(x)=0 \\
X_{n}^{\prime}(0)=0 \\
X_{n}^{\prime}(L)+H X_{n}(L)=0
\end{array}\right.
$$

que passaria a ter solução:

$$
X_{n}(x)=A_{n} \cos \zeta_{n} x
$$

onde os autovalores $\zeta_{n}$ seriam raízes de:

$$
\zeta_{n} \operatorname{sen} \zeta_{n} L-H \cos \zeta_{n} L=0
$$

que também não possui solução em forma fechada. 


\section{APÊNDICE B - O Método de Superposição}

O problema de valor de contorno e inicial:

$$
\left\{\begin{array}{l}
\frac{\partial^{2} T}{\partial x^{2}}+\frac{\partial^{2} T}{\partial y^{2}}+\frac{q^{\prime \prime \prime}(x, y)}{k}=\frac{1}{\alpha} \frac{\partial T}{\partial t}, \quad 0<x<L_{1}, 0<y<L_{2}, 0<t \\
-k_{1} \frac{\partial T}{\partial x}(0, y, t)+h_{1} T(0, y, t)=f_{1}(y), \quad 0<y<L_{2}, 0<t \\
k_{2} \frac{\partial T}{\partial x}\left(L_{1}, y, t\right)+h_{2} T\left(L_{1}, y, t\right)=f_{2}(y), \quad 0<y<L_{2}, 0<t \\
-k_{3} \frac{\partial T}{\partial y}(x, 0, t)+h_{3} T(x, 0, t)=f_{3}(x), \quad 0<x<L_{1}, 0<t \\
k_{4} \frac{\partial T}{\partial y}\left(x, L_{2}, t\right)+h_{4} T\left(x, L_{2}, t\right)=f_{4}(x), \quad 0<x<L_{1}, 0<t \\
T(x, y, 0)=f_{5}(x, y), \quad 0<x<L_{1}, 0<y<L_{2}
\end{array}\right.
$$

pode ser resolvido pela superposição das soluções de seis problemas auxiliares, cada uma com um dos correspondentes termos não homogêneos:

$$
T(x, y, t)=T_{1}(x)+T_{2}(x, y)+T_{3}(x, y)+T_{4}(x, y)+T_{5}(x, y)+T_{6}(x, y, t)
$$

A não-homogeneidade da equação governante está presente no primeiro problema auxiliar:

$$
\left\{\begin{array}{l}
\frac{d^{2} T_{1}}{d x^{2}}+\frac{q^{\prime \prime \prime}(x, y)}{k}=0, \quad 0<x<L_{1} \\
-k_{1} \frac{d T_{1}}{d x}(0)+h_{1} T_{1}(0)=0 \\
k_{2} \frac{d T_{1}}{d x}\left(L_{1}\right)+h_{2} T_{1}\left(L_{1}\right)=0
\end{array}\right.
$$

cuja solução pode ser obtida por integração direta. Note que apenas uma variável independente é necessária (foi escolhida $x$ ).

A não-homogeneidade das duas primeiras condições de contorno estão presentes nos dois problemas auxiliares:

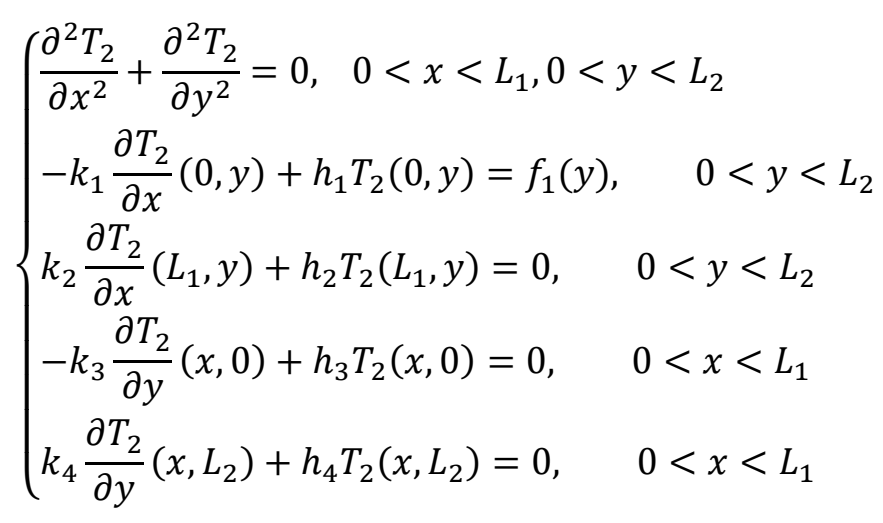

e 


$$
\left\{\begin{array}{l}
\frac{\partial^{2} T_{3}}{\partial x^{2}}+\frac{\partial^{2} T_{3}}{\partial y^{2}}=0, \quad 0<x<L_{1}, 0<y<L_{2} \\
-k_{1} \frac{\partial T_{3}}{\partial x}(0, y)+h_{1} T_{3}(0, y)=0, \quad 0<y<L_{2} \\
k_{2} \frac{\partial T_{3}}{\partial x}\left(L_{1}, y\right)+h_{2} T_{3}\left(L_{1}, y\right)=f_{2}(y), \quad 0<y<L_{2} \\
-k_{3} \frac{\partial T_{3}}{\partial y}(x, 0)+h_{3} T_{3}(x, 0)=0, \quad 0<x<L_{1} \\
k_{4} \frac{\partial T_{3}}{\partial y}\left(x, L_{2}\right)+h_{4} T_{3}\left(x, L_{2}\right)=0, \quad 0<x<L_{1}
\end{array}\right.
$$

cujas soluções podem ser obtidas pelo método da separação de variáveis.

A não-homogeneidade das duas últimas condições de contorno estão presentes nos dois problemas auxiliares:

$$
\left\{\begin{array}{l}
\frac{\partial^{2} T_{4}}{\partial x^{2}}+\frac{\partial^{2} T_{4}}{\partial y^{2}}=0, \quad 0<x<L_{1}, 0<y<L_{2} \\
-k_{1} \frac{\partial T_{4}}{\partial x}(0, y)+h_{1} T_{4}(0, y)=0, \quad 0<y<L_{2} \\
k_{2} \frac{\partial T_{4}}{\partial x}\left(L_{1}, y\right)+h_{2} T_{4}\left(L_{1}, y\right)=0, \quad 0<y<L_{2} \\
-k_{3} \frac{\partial T_{4}}{\partial y}(x, 0)+h_{3} T_{4}(x, 0)=f_{3}(x)-h_{3} T_{1}(x), \quad 0<x<L_{1} \\
k_{4} \frac{\partial T_{4}}{\partial y}\left(x, L_{2}\right)+h_{4} T_{4}\left(x, L_{2}\right)=0, \quad 0<x<L_{1}
\end{array}\right.
$$

e

$$
\begin{cases}\frac{\partial^{2} T_{5}}{\partial x^{2}}+\frac{\partial^{2} T_{5}}{\partial y^{2}}=0, \quad 0<x<L_{1}, 0<y<L_{2} \\ -k_{1} \frac{\partial T_{5}}{\partial x}(0, y)+h_{1} T_{5}(0, y)=0, \quad 0<y<L_{2} \\ k_{2} \frac{\partial T_{5}}{\partial x}\left(L_{1}, y\right)+h_{2} T_{5}\left(L_{1}, y\right)=0, \quad 0<y<L_{2} \\ -k_{3} \frac{\partial T_{5}}{\partial y}(x, 0)+h_{3} T_{5}(x, 0)=0, \quad 0<x<L_{1} \\ k_{4} \frac{\partial T_{5}}{\partial y}\left(x, L_{2}\right)+h_{4} T_{5}\left(x, L_{2}\right)=f_{4}(x)-h_{4} T_{1}(x), \quad 0<x<L_{1}\end{cases}
$$

cujas soluções podem ser obtidas pelo método da separação de variáveis. Note que são adicionados termos para compensar a solução acrescentada pelo primeiro problema.

Por fim, o termo transitório é incluído neste último problema auxiliar: 


$$
\left\{\begin{array}{l}
\frac{\partial^{2} T_{6}}{\partial x^{2}}+\frac{\partial^{2} T_{6}}{\partial y^{2}}=\frac{1}{\alpha} \frac{\partial T_{6}}{\partial t}, \quad 0<x<L_{1}, 0<y<L_{2}, 0<t \\
-k_{1} \frac{\partial T_{6}}{\partial x}(0, y, t)+h_{1} T_{6}(0, y, t)=0, \quad 0<y<L_{2}, 0<t \\
k_{2} \frac{\partial T_{6}}{\partial x}\left(L_{1}, y, t\right)+h_{2} T_{6}\left(L_{1}, y, t\right)=0, \quad 0<y<L_{2}, 0<t \\
-k_{3} \frac{\partial T_{6}}{\partial y}(x, 0, t)+h_{4} T_{6}(x, 0, t)=0, \quad 0<x<L_{1}, 0<t \\
k_{4} \frac{\partial T_{6}}{\partial y}\left(x, L_{2}, t\right)+h_{4} T_{6}\left(x, L_{2}, t\right)=0, \quad 0<x<L_{1}, 0<t \\
T_{6}(x, y, 0)=f_{5}(x, y)-T_{1}(x)-T_{2}(x, y)-T_{3}(x, y)-T_{4}(x, y)-T_{5}(x, y), 0<x<L_{1}, 0<y<L_{2}
\end{array}\right.
$$

cuja solução pode ser obtida pelo método da separação de variáveis. Note que são adicionados termos para compensar as soluções acrescentadas pelos problemas auxiliares anteriores.

É fácil provar que a soma dessas soluções leva a solução do problema original. A equação governante é satisfeita pela soma desses problemas auxiliares, pois:

$$
\begin{gathered}
\frac{\partial^{2} T}{\partial x^{2}}+\frac{\partial^{2} T}{\partial y^{2}}+\frac{q^{\prime \prime \prime}(x, y)}{k}= \\
=\frac{\partial^{2}}{\partial x^{2}}\left(T_{1}(x)+T_{2}(x, y)+T_{3}(x, y)+T_{4}(x, y)+T_{5}(x, y)+T_{6}(x, y, t)\right)+ \\
+\frac{\partial^{2}}{\partial y^{2}}\left(T_{1}(x)+T_{2}(x, y)+T_{3}(x, y)+T_{4}(x, y)+T_{5}(x, y)+T_{6}(x, y, t)\right)+\frac{g(x, y)}{k}= \\
=\left(\frac{d^{2} T_{1}}{d x^{2}}+\frac{g(x, y)}{k}\right)+\left(\frac{\partial^{2} T_{2}}{\partial x^{2}}+\frac{\partial^{2} T_{2}}{\partial y^{2}}\right)+\left(\frac{\partial^{2} T_{3}}{\partial x^{2}}+\frac{\partial^{2} T_{3}}{\partial y^{2}}\right)+ \\
+\left(\frac{\partial^{2} T_{4}}{\partial x^{2}}+\frac{\partial^{2} T_{4}}{\partial y^{2}}\right)+\left(\frac{\partial^{2} T_{5}}{\partial x^{2}}+\frac{\partial^{2} T_{5}}{\partial y^{2}}\right)+\left(\frac{\partial^{2} T_{6}}{\partial x^{2}}+\frac{\partial^{2} T_{6}}{\partial y^{2}}\right)= \\
=-\frac{1}{\alpha} \frac{\partial}{\partial t}\left(\left(T_{1}(x)+T_{2}(x, y)+T_{3}(x, y)+T_{4}(x, y)+T_{5}(x, y)+T_{6}(x, y, t)\right)\right)=\frac{1}{\alpha} \frac{\partial T}{\partial t}
\end{gathered}
$$

A primeira condição de contorno também é satisfeita pela soma desses problemas auxiliares, pois:

$$
\begin{gathered}
-k_{1} \frac{\partial}{\partial x} T(0, y, t)+h_{1} T(0, y, t)= \\
=-k_{1} \frac{\partial}{\partial x}\left(T_{1}(0)+T_{2}(0, y)+T_{3}(0, y)+T_{4}(0, y)+T_{5}(0, y)+T_{6}(0, y, t)\right)+ \\
+h_{1}\left(T_{1}(0)+T_{2}(0, y)+T_{3}(0, y)++T_{4}(0, y)+T_{5}(0, y)+T_{6}(0, y, t)\right)= \\
=\left(-k_{1} \frac{\partial}{\partial x} T_{1}(0)+h_{1} T_{1}(0)\right)+\left(-k_{1} \frac{\partial}{\partial x} T_{2}(0, y)+h_{1} T_{2}(0, y)\right)+ \\
+\left(-k_{1} \frac{\partial}{\partial x} T_{3}(0, y)+h_{1} T_{3}(0, y)\right)+\left(-k_{1} \frac{\partial}{\partial x} T_{4}(0, y)+h_{1} T_{4}(0, y)\right)+
\end{gathered}
$$




$$
+\left(-k_{1} \frac{\partial}{\partial x} T_{5}(0, y)+h_{1} T_{5}(0, y)\right)+\left(-k_{1} \frac{\partial}{\partial x} T_{6}(0, y, t)+h_{1} T_{6}(0, y, t)\right)=f_{1}(y)
$$

As demais condições de contorno também são satisfeitas de forma análoga, até a quarta condição de contorno:

$$
\begin{gathered}
k_{4} \frac{\partial}{\partial x} T\left(x, L_{2}, t\right)+h_{4} T\left(x, L_{2}, t\right)= \\
=k_{4} \frac{\partial}{\partial x}\left(T_{1}(x)+T_{2}\left(x, L_{2}\right)+T_{3}\left(x, L_{2}\right)+T_{4}\left(x, L_{2}\right)+T_{5}\left(x, L_{2}\right)+T_{6}\left(x, L_{2}, t\right)\right)+ \\
+h_{4}\left(T_{1}(x)+T_{2}\left(x, L_{2}\right)+T_{3}\left(x, L_{2}\right)+T_{4}\left(x, L_{2}\right)+T_{5}\left(x, L_{2}\right)+T_{6}\left(x, L_{2}, t\right)\right)= \\
=\left(k_{4} \frac{\partial}{\partial y} T_{1}(x)+h_{4} T_{1}(x)\right)+\left(k_{4} \frac{\partial}{\partial y} T_{2}\left(x, L_{2}\right)+h_{4} T_{2}\left(x, L_{2}\right)\right)+ \\
+\left(k_{4} \frac{\partial}{\partial y} T_{3}\left(x, L_{2}\right)+h_{4} T_{3}\left(x, L_{2}\right)\right)+\left(k_{4} \frac{\partial}{\partial y} T_{4}\left(x, L_{2}\right)+h_{4} T_{4}\left(x, L_{2}\right)\right)+ \\
+\left(k_{4} \frac{\partial}{\partial y} T_{5}\left(x, L_{2}\right)+h_{4} T_{5}\left(x, L_{2}\right)\right)+\left(k_{4} \frac{\partial}{\partial y} T_{6}\left(x, L_{2}, t\right)+h_{4} T_{6}\left(x, L_{2}, t\right)\right) \\
=+h_{4} T_{1}(x)+f_{4}(x)-h_{4} T_{1}(x)=f_{4}(x)
\end{gathered}
$$

Por fim, a condição inicial também será satisfeita:

$$
\begin{gathered}
T(x, y, 0)=T_{1}(x)+T_{2}(x, y)+T_{3}(x, y)+T_{4}(x, y)+T_{5}(x, y)+T_{6}(x, y, 0)= \\
=T_{1}(x)+T_{2}(x, y)+T_{3}(x, y)+T_{4}(x, y)+T_{5}(x, y)+ \\
+f_{5}(x, y)-T_{1}(x)-T_{2}(x, y)-T_{3}(x, y)-T_{4}(x, y)-T_{5}(x, y)=f_{5}(x, y)
\end{gathered}
$$




\section{APÊNDICE C - A Função de Green}

Morse e Feshback (1953) mostram que o problema de valor de contorno e inicial:

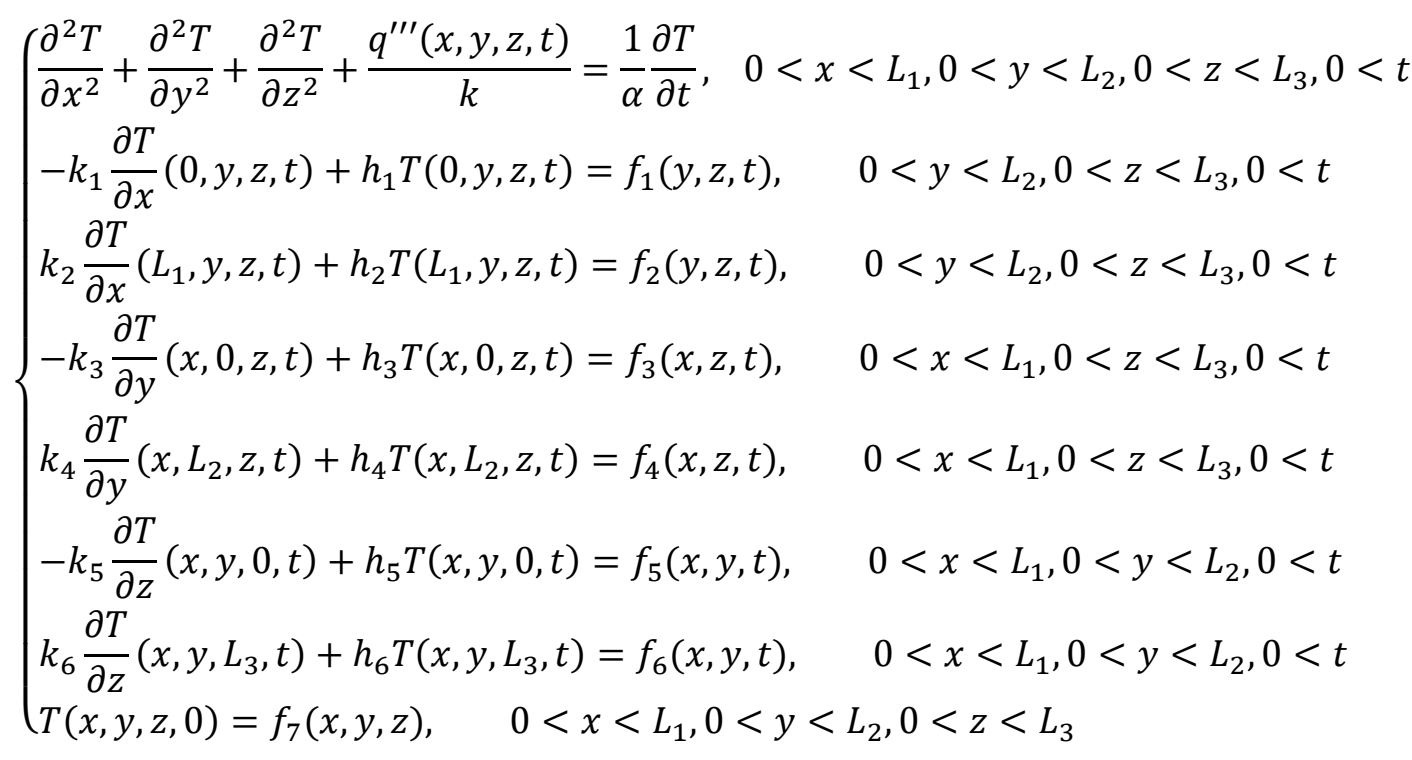

tem solução

$$
\begin{gathered}
T(x, y, z, t)=\left.\int_{z^{\prime}=0}^{L_{3}} \int_{y^{\prime}=0}^{L_{2}} \int_{x^{\prime}=0}^{L_{1}} G\left(x, y, z, t \mid x^{\prime}, y^{\prime}, z^{\prime}, \tau\right)\right|_{\tau=0} f_{7}\left(x^{\prime}, y^{\prime}, z^{\prime}\right) d x^{\prime} d y^{\prime} d z^{\prime} \\
+\frac{\alpha}{k} \int_{\tau=0}^{t} \int_{z^{\prime}=0}^{L_{3}} \int_{y^{\prime}=0}^{L_{2}} \int_{x^{\prime}=0}^{L_{1}} G\left(x, y, z, t \mid x^{\prime}, y^{\prime}, z^{\prime}, \tau\right) q^{\prime \prime \prime}\left(x^{\prime}, y^{\prime}, z^{\prime}, \tau\right) d x^{\prime} d y^{\prime} d z^{\prime} d \tau \\
+\left.\alpha \int_{\tau=0}^{t} \int_{z^{\prime}=0}^{L_{3}} \int_{y^{\prime}=0}^{L_{2}} G\left(x, y, z, t \mid 0, y^{\prime}, z^{\prime}, \tau\right)\right|_{\tau=0} \frac{1}{k_{1}} f_{1}\left(y^{\prime}, z^{\prime}, \tau\right) d y d z d \tau \\
+\left.\alpha \int_{\tau=0}^{t} \int_{z^{\prime}=0}^{L_{3}} \int_{y^{\prime}=0}^{L_{2}} G\left(x, y, z, t \mid L_{1}, y^{\prime}, z^{\prime}, \tau\right)\right|_{\tau=0} \frac{1}{k_{2}} f_{2}\left(y^{\prime}, z^{\prime}, \tau\right) d y d z d \tau+\cdots \\
+\left.\alpha \int_{\tau=0}^{t} \int_{y^{\prime}=0}^{L_{2}} \int_{x^{\prime}=0}^{L_{1}} G\left(x, y, z, t \mid x^{\prime}, y^{\prime}, L_{3}, \tau\right)\right|_{\tau=0} \frac{1}{k_{6}} f_{6}\left(x^{\prime}, y^{\prime}, \tau\right) d y d z d \tau
\end{gathered}
$$

onde a função de Green $G$ representa a temperatura no ponto $(x, y, z)$, no instante $t$, devido a uma fonte de energia localizada no ponto $\left(x^{\prime}, y^{\prime}, z^{\prime}\right)$ liberando energia espontaneamente no tempo $t=\tau$, e satisfazendo condições de contorno homogêneas: 


$$
\begin{cases}\frac{\partial^{2} G}{\partial x^{2}}+\frac{\partial^{2} G}{\partial y^{2}}+\frac{\partial^{2} G}{\partial z^{2}}+\frac{\delta\left(x-x^{\prime}, y-y^{\prime}, z-z^{\prime}\right) \delta(t-\tau)}{k}=\frac{1}{\alpha} \frac{\partial G}{\partial t}, 0<x<L_{1}, 0<y<L_{2}, 0<z<L_{3}, \tau<t \\ -k_{1} \frac{\partial G}{\partial x}(0, y, z, t)+h_{1} G(0, y, z, t)=0, & 0<y<L_{2}, 0<z<L_{3}, \tau<t \\ k_{2} \frac{\partial G}{\partial x}\left(L_{1}, y, z, t\right)+h_{2} G\left(L_{1}, y, z, t\right)=0, & 0<y<L_{2}, 0<z<L_{3}, \tau<t \\ -k_{3} \frac{\partial G}{\partial y}(x, 0, z, t)+h_{3} G(x, 0, z, t)=0, & 0<x<L_{1}, 0<z<L_{3}, \tau<t \\ k_{4} \frac{\partial G}{\partial y}\left(x, L_{2}, z, t\right)+h_{4} G\left(x, L_{2}, z, t\right)=0, & 0<x<L_{1}, 0<z<L_{3}, \tau<t \\ -k_{5} \frac{\partial G}{\partial z}(x, y, 0, t)+h_{5} G(x, y, 0, t)=0, & 0<x<L_{1}, 0<y<L_{2}, \tau<t \\ k_{6} \frac{\partial G}{\partial z}\left(x, y, L_{3}, t\right)+h_{6} G\left(x, y, L_{3}, t\right)=0, & 0<x<L_{1}, 0<y<L_{2}, \tau<t\end{cases}
$$

Hahn e Özişik (2012) mostra um modo bem mais simples de se resolver esse problema auxiliar do que Morse e Feshback (1953) e, posteriormente, Carslaw e Jaeger (1959) apresentaram. $G\left(x, y, z, t \mid x^{\prime}, y^{\prime}, z^{\prime}, \tau\right)$ pode ser obtida simplesmente substituindo $t$ por $t-\tau$ em $G\left(x, y, z, t \mid x^{\prime}, y^{\prime}, z^{\prime}, 0\right)$.

Então, considerando o problema:

$$
\left\{\begin{array}{lc}
\frac{\partial^{2} \psi}{\partial x^{2}}+\frac{\partial^{2} \psi}{\partial y^{2}}+\frac{\partial^{2} \psi}{\partial z^{2}}=\frac{1}{\alpha} \frac{\partial \psi}{\partial t}, \quad 0<x<L_{1}, 0<y<L_{2}, 0<z<L_{3}, \tau<t \\
-k_{1} \frac{\partial \psi}{\partial x}(0, y, z, t)+h_{1} \psi(0, y, z, t)=0, & 0<y<L_{2}, 0<z<L_{3}, \tau<t \\
k_{2} \frac{\partial \psi}{\partial x}\left(L_{1}, y, z, t\right)+h_{2} \psi\left(L_{1}, y, z, t\right)=0, & 0<y<L_{2}, 0<z<L_{3}, \tau<t \\
-k_{3} \frac{\partial \psi}{\partial y}(x, 0, z, t)+h_{3} \psi(x, 0, z, t)=0, & 0<x<L_{1}, 0<z<L_{3}, \tau<t \\
k_{4} \frac{\partial \psi}{\partial y}\left(x, L_{2}, z, t\right)+h_{4} \psi\left(x, L_{2}, z, t\right)=0, & 0<x<L_{1}, 0<z<L_{3}, \tau<t \\
-k_{5} \frac{\partial \psi}{\partial z}(x, y, 0, t)+h_{5} \psi(x, y, 0, t)=0, & 0<x<L_{1}, 0<y<L_{2}, \tau<t \\
k_{6} \frac{\partial \psi}{\partial z}\left(x, y, L_{3}, t\right)+h_{6} \psi\left(x, y, L_{3}, t\right)=0, & 0<x<L_{1}, 0<y<L_{2}, \tau<t \\
\psi(x, y, z, 0)=f_{7}(x, y, z), \quad 0<x<L_{1}, 0<y<L_{2}, 0<z<L_{3}
\end{array}\right.
$$

e utilizando o método da separação de variáveis:

$$
\psi(x, y, z, t)=\sum_{m} \sum_{n} \sum_{p} X_{m}(x) Y_{n}(y) Z_{p}(z) \Gamma(t)
$$

obtém-se

$$
\frac{X_{m}^{\prime \prime}(x)}{X_{m}(x)}+\frac{Y_{n}^{\prime \prime}(y)}{Y_{n}(y)}+\frac{Z_{p}^{\prime \prime}(z)}{Z_{p}(z)}=\frac{1}{\alpha} \frac{\Gamma^{\prime}(t)}{\Gamma(t)}
$$

Assim: 


$$
\left\{\begin{array} { l } 
{ X _ { m } ^ { \prime \prime } ( x ) + \zeta _ { m } ^ { 2 } X _ { m } ( x ) = 0 } \\
{ Y _ { n } ^ { \prime \prime } ( y ) + \gamma _ { n } ^ { 2 } Y _ { n } ( y ) = 0 } \\
{ Z _ { p } ^ { \prime \prime } ( z ) + \eta _ { p } ^ { 2 } Z _ { p } ( z ) = 0 } \\
{ \Gamma ^ { \prime } ( t ) + \alpha ( \zeta _ { m } ^ { 2 } + \gamma _ { n } ^ { 2 } + \eta _ { p } ^ { 2 } ) \Gamma ( t ) = 0 }
\end{array} \rightarrow \left\{\begin{array}{l}
X_{m}(x)=A_{m} \operatorname{sen} \zeta_{m} x+B_{m} \cos \zeta_{m} x \\
Y_{n}(y)=C_{n} \operatorname{sen} \gamma_{n} y+D_{n} \cos \gamma_{n} y \\
Z_{p}(z)=E_{p} \operatorname{sen} \gamma_{p} z+F_{p} \cos \gamma_{p} z \\
\Gamma(t)=e^{-\alpha\left(\zeta_{m}^{2}+\gamma_{n}^{2}+\eta_{p}^{2}\right) t}
\end{array}\right.\right.
$$

onde as constantes e os autovalores são determinados pelas condições de contorno, ou seja, os autovalores vão satisfazer as mesmas equações obtidas pelos outros métodos.

Portanto:

$$
\psi(x, y, z, t)=\sum_{m} \sum_{n} \sum_{p} c_{m n p} e^{-\alpha\left(\zeta_{m}^{2}+\gamma_{n}^{2}+\eta_{p}^{2}\right) t} X_{m}(x) Y_{n}(y) Z_{p}(z)
$$

A condição inicial leva a

$$
\psi(x, y, z, 0)=\sum_{m} \sum_{n} \sum_{p} c_{m n p} X_{m}(x) Y_{n}(y) Z_{p}(z)=f_{7}(x, y, z)
$$

Aplicando-se a ambos lados da equação, sucessivamente, os operadores

$$
\int_{0}^{L_{1}} X_{m}(x) d x, \quad \int_{0}^{L_{1}} Y_{n}(y) d y, \quad \int_{0}^{L_{1}} Z_{p}(z) d z
$$

obtém-se

$$
c_{m n p}=\frac{1}{N\left(\zeta_{m}\right) N\left(\gamma_{n}\right) N\left(\eta_{p}\right)} \int_{0}^{L_{3}} \int_{0}^{L_{2}} \int_{0}^{L_{1}} X_{m}\left(x^{\prime}\right) Y_{n}\left(y^{\prime}\right) Z_{p}\left(z^{\prime}\right) f_{7}(x, y, z) d x^{\prime} d y^{\prime} d z^{\prime}
$$

onde

$$
\begin{aligned}
& N\left(\beta_{m}\right)=\int_{0}^{L_{1}} X_{m}^{2}(x) d x \\
& N\left(\gamma_{n}\right)=\int_{0}^{L_{1}} Y_{n}^{2}(y) d y \\
& N\left(\eta_{p}\right)=\int_{0}^{L_{1}} Z_{p}^{2}(z) d z
\end{aligned}
$$

Agora resolve-se esse problema novamente por função de Green:

$$
\begin{gathered}
\psi(x, y, z, t)=\left.\int_{z^{\prime}=0}^{L_{3}} \int_{y^{\prime}=0}^{L_{2}} \int_{x^{\prime}=0}^{L_{1}} G\left(x, y, z, t \mid x^{\prime}, y^{\prime}, z^{\prime}, \tau\right)\right|_{\tau=0} f_{7}\left(x^{\prime}, y^{\prime}, z^{\prime}\right) d x^{\prime} d y^{\prime} d z^{\prime} \\
+\left.\frac{\alpha}{k} \int_{\tau=0}^{t} \int_{z^{\prime}=0}^{L_{3}} \int_{y^{\prime}=0}^{L_{2}} \int_{x^{\prime}=0}^{L_{1}} G\left(x, y, z, t \mid x^{\prime}, y^{\prime}, z^{\prime}, \tau\right)\right|_{\tau=0} 0 d x^{\prime} d y^{\prime} d z^{\prime} d \tau \\
+\left.\alpha \int_{\tau=0}^{t} \int_{z^{\prime}=0}^{L_{3}} \int_{y^{\prime}=0}^{L_{2}} G\left(x, y, z, t \mid 0, y^{\prime}, z^{\prime}, \tau\right)\right|_{\tau=0} 0 d y d z d \tau \\
+\left.\alpha \int_{\tau=0}^{t} \int_{z^{\prime}=0}^{L_{3}} \int_{y^{\prime}=0}^{L_{2}} G\left(x, y, z, t \mid L_{1}, y^{\prime}, z^{\prime}, \tau\right)\right|_{\tau=0} 0 d y d z d \tau+\cdots
\end{gathered}
$$




$$
\begin{gathered}
+\left.\alpha \int_{\tau=0}^{t} \int_{y^{\prime}=0}^{L_{2}} \int_{x^{\prime}=0}^{L_{1}} G\left(x, y, z, t \mid x^{\prime}, y^{\prime}, L_{3}, \tau\right)\right|_{\tau=0} 0 d y d z d \tau \\
=\left.\int_{z^{\prime}=0}^{L_{3}} \int_{y^{\prime}=0}^{L_{2}} \int_{x^{\prime}=0}^{L_{1}} G\left(x, y, z, t \mid x^{\prime}, y^{\prime}, z^{\prime}, \tau\right)\right|_{\tau=0} f_{7}\left(x^{\prime}, y^{\prime}, z^{\prime}\right) d x^{\prime} d y^{\prime} d z^{\prime}
\end{gathered}
$$

Comparando-se as duas soluções, nota-se que:

$$
\left.G\left(x, y, z, t \mid x^{\prime}, y^{\prime}, z^{\prime}, \tau\right)\right|_{\tau=0}=\frac{e^{-\alpha\left(\zeta_{m}^{2}+\gamma_{n}^{2}+\eta_{p}^{2}\right) t} X_{m}(x) Y_{n}(y) Z_{p}(z) X_{m}\left(x^{\prime}\right) Y_{n}\left(y^{\prime}\right) Z_{p}\left(z^{\prime}\right)}{N\left(\beta_{m}\right) N\left(\gamma_{n}\right) N\left(\eta_{p}\right)}
$$

Então, substituindo $t$ por $t-\tau$ :

$$
G\left(x, y, z, t \mid x^{\prime}, y^{\prime}, z^{\prime}, \tau\right)=\frac{e^{-\alpha\left(\zeta_{m}^{2}+\gamma_{n}^{2}+\eta_{p}^{2}\right)(t-\tau)} X_{m}(x) Y_{n}(y) Z_{p}(z) X_{m}\left(x^{\prime}\right) Y_{n}\left(y^{\prime}\right) Z_{p}\left(z^{\prime}\right)}{N\left(\beta_{m}\right) N\left(\gamma_{n}\right) N\left(\eta_{p}\right)}
$$

o que completa a solução. 